\title{
Grenzflächeneffekte in Manganatschichten
}

\author{
Dissertation \\ zur Erlangung des Doktorgrades \\ der Mathematisch-Naturwissenschaftlichen Fakultäten \\ der Georg-August-Universität zu Göttingen
}

vorgelegt von

Markus Eßeling

aus Bad Laer

Göttingen 2007 
Referent

Korreferent

Tag der mündlichen Prüfung:
Prof. Dr. Konrad Samwer PD Dr. Christian Jooss

10. Oktober 2007 
Sie befinden sich alle in derselben Ebene. Alle kreisen in derselben Richtung... Es ist vollkommen, wissen Sie. Es ist großartig. Es ist fast unheimlich. (Der Astronom Geoffrey Marcy über das Sonnensystem) 



\section{Inhaltsverzeichnis}

Titel i

Inhaltsverzeichnis vii

1 Einleitung 1

2 Manganate 5

2.1 Struktur der Manganate . . . . . . . . . . . . . . . 6 6

2.2 Elektronische Struktur . . . . . . . . . . . . . . . 8

2.2.1 Elektronische Struktur von $\mathrm{LaMnO}_{3} \ldots \ldots \ldots \ldots$

2.2 .2 Jahn-Teller-Effekt . . . . . . . . . . . . . 8

2.2.3 Austauschmechanismen . . . . . . . . . . . . . . 10

2.2.4 Das Phasendiagramm $\mathrm{La}_{1-x} \mathrm{Sr}_{x} \mathrm{MnO}_{3} \ldots \ldots \ldots \ldots$

2.3 Elektronische Phasenseparation . . . . . . . . . . . . . 15

2.4 Einfluss von Polaronen . . . . . . . . . . . . . . . 16

2.5 Extrinsische Effekte . . . . . . . . . . . . . . . 17

2.5.1 Dünne Schichten ................... 17

2.5.2 Dotierung von Korngrenzen in $\mathrm{La}_{0,7} \mathrm{Sr}_{0,3} \mathrm{MnO}_{3} \ldots \ldots \ldots$. . . . 18

2.6 Widerstandsschalten in Perowskiten . . . . . . . . . . . . 19

3 Magnetowiderstandseffekte 21

3.1 Der Tunneleffekt . . . . . . . . . . . . . . . . . . 22

3.1.1 Elastisches Tunneln durch eine eindimensionale, rechteckige Poten-

tialbarriere ......................... 22

3.1.2 Erweiterung auf den dreidimensionalen Fall . . . . . . . . . 24

3.1.3 Elastisches Tunneln in planaren Tunnelkontakten . . . . . . . . 24

3.1.4 Temperatur- und Bandstruktureffekte . . . . . . . . . . 25

3.2 Tunnel-Magnetowiderstand . . . . . . . . . . . . . . 26 26

3.2 .1 Jullière-Modell . . . . . . . . . . . . . . . . . . . 26 26

3.2.2 Erweiterungen des Jullière-Modells . . . . . . . . . . . . . 28

3.2.3 Temperatur- und Spannungsabhängigkeit . . . . . . . . . . . . . 29

3.3 Strominduziertes Magnetisierungsschalten . . . . . . . . . . . . 31

3.4 Korngrenzen-Magnetowiderstand in Manganaten . . . . . . . . . . . . 34 
4 Rauschen 37

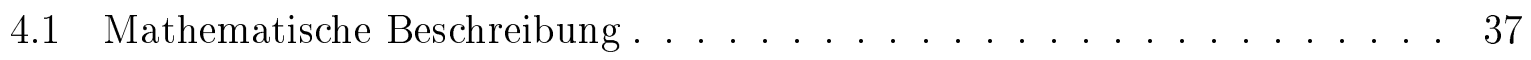

4.2 Thermisches Rauschen . . . . . . . . . . . . . . . . 38

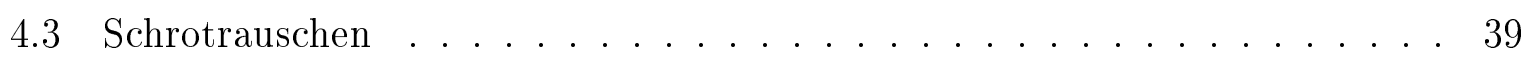

$4.41 / f$-Widerstandsrauschen . . . . . . . . . . . . . . . . 39

4.4.1 Allgemeine Beschreibung . . . . . . . . . . . . . . . 39 . . . . . . . . . . . .

4.4 Hooge-Parametrisierung . . . . . . . . . . . . . 40

4.4.3 Modell für $1 / f$-Widerstandsrauschen . . . . . . . . . . . 40

4.4.4 $1 / f$-Widerstandsrauschen in Manganaten und TMR-Elementen . . 43

5 Experimentelle Techniken

5.1 Probenpräparation .......................... 45

5.1 Metallorganische Aerosol-Deposition . . . . . . . . . . 45

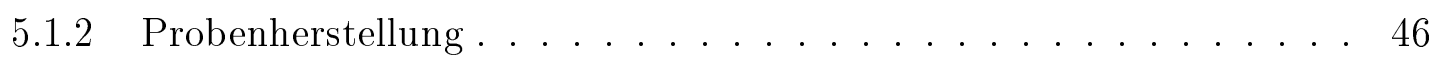

5.2 Röntgenstrukturanalyse . . . . . . . . . . . . . . 47

5.2 .1 Kleinwinkelanalyse . . . . . . . . . . . . . . . . 48

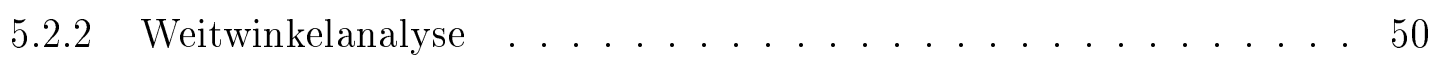

5.3 SQUID-Magnetometrie . . . . . . . . . . . . . . . . 51

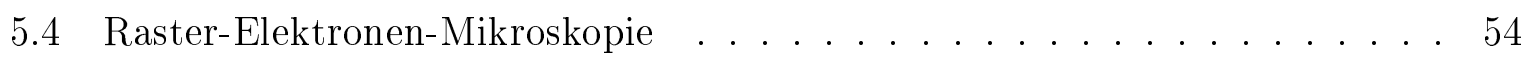

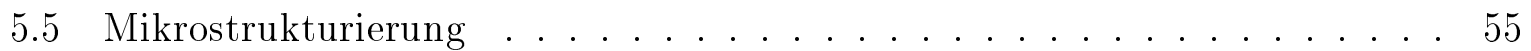

5.5.1 Von der Probe zur Struktur . . . . . . . . . . . . 56

5.5.2 Minimierung der Strukturgrößen unter Verwendung eines fokussierten Ionenstrahls . . . . . . . . . . . . . . . . 59

5.6 Transmissions-Elektronen-Mikroskopie . . . . . . . . . . . . . . . . . . . . . . .

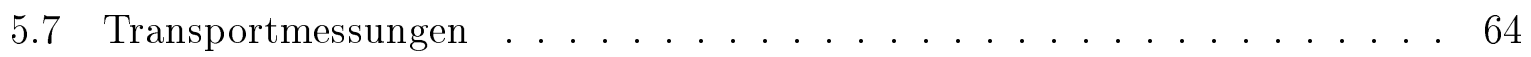

$5.81 / f$-Widerstandsrauschen . . . . . . . . . . . . . . 65

6 Nanokomposite 69

6.1 LSMO:MgO Nanokomposite . . . . . . . . . . . . . . . 69

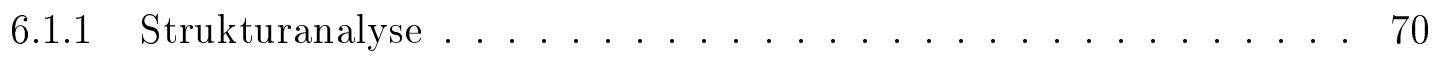

6.1 .2 Magnetotransport ................... 70

$6.1 .31 / f$-Widerstandsrauschen ................... 72

6.1 .4 Diskussion . . . . . . . . . . . . . . . 75

6.2 LSMO: $\mathrm{CeO}_{2}$ Nanokomposite . . . . . . . . . . . . . . 78

6.2.1 Strukturanalyse . . . . . . . . . . . . . 78

6.2.2 Magnetotransport . . . . . . . . . . . . . . . 79

6.2.3 Einfluss der Strukturgröße . . . . . . . . . . . . . . . . 81

6.2 .4 Diskussion . . . . . . . . . . . . . . . . 82

6.3 Nanokristallines LSMO . . . . . . . . . . . . . . . . . . . . . . . . . . . . . . . .

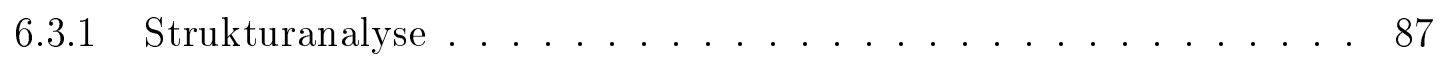

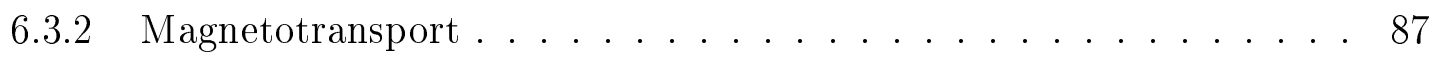

6.3.3 Strominduzierte Effekte . . . . . . . . . . . 88

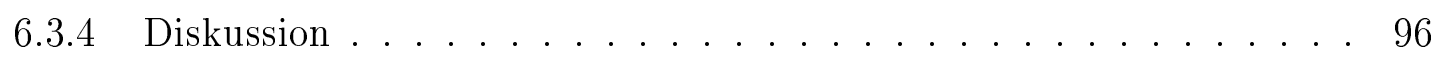


7 Bikristallkontakte 111

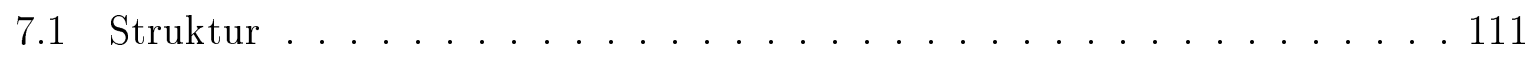

7.2 Magnetotransport . . . . . . . . . . . . . . . . . 115

7.2.1 LSMO Korngrenzen-Kontakte . . . . . . . . . . . . . . . . . . . . . . . . . . . . . .

7.2.2 LSMO:MgO Korngrenzen-Kontakte . . . . . . . . . . . . . 120

7.2.3 LSMO: $\mathrm{CeO}_{2}$ Korngrenzen-Kontakte . . . . . . . . . . . . . 126

7.2.4 Zusammenfassung Magnetotransport . . . . . . . . . . . . . . . 129

7.3 Strominduzierte Effekte . . . . . . . . . . . . . . . . 129

7.3.1 LSMO:MgO Korngrenzen-Kontakte . . . . . . . . . . . . . . 129

7.4 Diskussion . . . . . . . . . . . . . . . . . . . 131

7.4 .1 Struktur ............................ 131

7.4 .2 Magnetotransport ....................... 134

7.4.3 Strominduzierte Effekte . . . . . . . . . . . . . 144

8 Zusammenführende Diskussion 147

8.1 Struktur . . . . . . . . . . . . . . . . . . . . . 147

8.2 Magnetotransport . . . . . . . . . . . . . . . . . . . . 148

8.3 Strom-/Spannungsinduzierte Effekte . . . . . . . . . . . . 151

9 Zusammenfassung und Ausblick 153

9.1 Zusammenfassung . . . . . . . . . . . . . . . . . 153

9.2 Ausblick . . . . . . . . . . . . . . . . . . . 156

Literaturverzeichnis 157

Publikationen 173

Danksagung

Lebenslauf 



\section{Kapitel 1}

\section{Einleitung}

Magnetische Bauelemente sind aus unserem täglichen Leben nicht mehr wegzudenken. Sie befinden sich in Transformatoren, Sensoren und Motoren sowie in vielen weiteren alltäglichen Anwendungen. Auch in die moderne Informationstechnologie haben sie Einzug gehalten. Die Verwendung magnetischer Speicherelemente verspricht einen wesentlichen Fortschritt, da durch die zusätzliche Nutzung des Spinfreiheitsgrades des Elektrons Vorteile gegenüber der nur auf der Ladung basierenden, konventionellen Halbleiterelektronik entstehen [1]. Solche Speicherelemente beruhen meist auf magnetoresistiven Effekten, also der Änderung des elektrischen Widerstandes durch Variation eines äußeren Feldes. Dabei können die physikalischen Mechanismen vielfältig sein. Ein wichtiger Vertreter ist der Tunnel-Magnetowiderstandseffekt (engl.: tunneling magnetoresistance, TMR). Dieser beschreibt die Abhängigkeit des Tunnelstroms zwischen zwei, durch eine dünne Isolationsschicht getrennten, ferromagnetischen Elektroden in Abhängigkeit der relativen Orientierung ihrer Magnetisierungen [2,3]. Die beobachteten Widerstandsänderungen können dabei auch bei Raumtemperatur sehr hoch sein [4.5] und eignen sich daher zum Einsatz in nichtflüchtigen Speicherelementen.

Von großer Bedeutung für die Anwendung solcher magnetoresistiven Effekte ist ihre Umkehrung: Durch ausreichend hohe Stromdichten definierter Polarität kann die relative Orientierung der Magnetisierungen auch ohne äußeres Feld geändert werden [6,7,8,8, 9 . Der Notwendigkeit hoher Stromdichten kommt der andauernde Ruf nach Miniaturisierung in der Informationstechnologie entgegen, da durch die Minimierung der Strukturgrößen auch die Stromdichten erhöht werden können. Dennoch stößt die konventionelle Nanostrukturierung für solche Effekte an ihre Grenze und die Entwicklung neuartiger Konzepte ist notwendig. Eine mögliche Lösung liegt in der Verwendung von selbstorganisierten, lateralen Systemen, wodurch die komplexe, vertikale Strukturierung umgangen werden kann. Solche Systeme bieten nicht zuletzt aufgrund ihrer gegenüber Dünnschicht-Heterostrukturen deutlich vereinfachten Präparation große Vorteile. Dennoch sind viele grundlegende, physikalische Eigenschaften unverstanden. Insbesondere die in weiten Teilen unbekannten Grenzflächeneigenschaften sind in solchen Systemen von großer Bedeutung, da sie maßgeblich für die Widerstandsänderungen sind. Auch der Einfluss der Anzahl der in den Prozess involvierten Grenzflächen im Vergleich zu den Eigenschaften einer einzelnen Grenzfläche ist weitgehend ungeklärt. Ebenso ist das strominduzierte Magnetisierungsschalten in la- 
teralen Systemen ungeklärt und bedarf systematischer Untersuchungen.

Die Verwendung von Manganaten in solch selbstorganisierten, magnetoresistiven Strukturen ermöglicht aufgrund ihrer hohen Spinpolarisation [10] ein tieferes Verständnis der dominanten Mechanismen. Dabei üben Manganate schon an sich aufgrund ihrer vielfältigen physikalischen Eigenschaften eine große Faszination aus [11|. Infolge konkurrierender Wechselwirkungen unterschiedlicher Stärke kommt es beispielsweise zu verschiedenen elektronischen Grundzuständen und magnetischen Ordnungen 12. Entsprechend sind die elektronischen Eigenschaften dieser Materialien stark von äußeren Feldern abhängig. Dazu zählen der kolossale Magnetowiderstand (engl.: colossal magnetoresistance, CMR) in magnetischen Feldern nahe der Curie-Temperatur [13], der kolossale Elektrowiderstand (engl.: colossal electroresistance, CER) in elektrischen Feldern [14 oder auch Widerstandsänderungen unter optischen [15] und elastischen Einflüssen.

Die großen Widerstandsänderungen der Manganate in verschiedenen äußeren Feldern werden oft im Zusammenhang mit der elektronischen Phasenseparation diskutiert. Sie beschreibt die Ausbildung unterschiedlicher elektronischer Bereiche im chemisch homogenen Material [16, 17]. Durch dieses Konzept, welches vielfältige experimentelle Evidenz hat [18, 19, 20, 21], können die beobachteten Widerstandsänderungen besser quantitativ beschrieben werden. Zusätzlich zur elektronischen Phasenseparation werden polaronische Effekte im Zusammenhang mit den elektronischen Eigenschaften diskutiert [22,23]. Eine Verbindung der beiden Konzepte kann zum wesentlichen Verständnis der Physik der Manganate [24] und gegebenenfalls weiterer, stark korrelierter Elektronensysteme wie der Hochtemperatursupraleiter beitragen.

Von großem Interesse für mögliche Anwendungen ist das durch elektrische Spannungspulse induzierte Widerstandsschalten (engl.: electric pulse induced resistance, EPIR) in isolierenden Manganaten und weiteren Perowskiten 25,26]. Hier werden Widerstandsänderungen von einigen Größenordnungen erzielt, welche über mehrere Monate stabil sind. Die zugrundeliegenden physikalischen Mechanismen werden in der Literatur kontrovers diskutiert. In speziellen Systemen konnte jedoch ein direkter Zusammenhang mit polaronischen Effekten und der elektronischen Phasenseparation nachgewiesen werden 22.

Die Präparation selbstorganisierter, lateraler Magnetowiderstandssysteme auf Basis der Manganate bietet entsprechend nicht nur eine hervorragende Möglichkeit zur grundlegenden Untersuchung der oben beschriebenen Charakteristika von magnetischen Bauelementen, sondern zusätzlich durch eine Kombination ihrer spezifischen Merkmale, wie beispielsweise dem EPIR, mit den magnetoresistiven Eigenschaften (TMR) die Möglichkeit zur Konstruktion neuartiger Speicherkonzepte. Es ergeben sich neue Wege in der Nanotechnologie durch Effekte, welche auf unterschiedlichen äußeren Feldern basieren.

Ein mögliches System stellen die nanokristallinen Manganate in Dünnschichtform dar. Über die Präparationsbedingungen wie Wahl des Substrats sowie der Depositionsbedingungen (Temperatur, Wachstumsrate etc.) kann gezielt Einfluss auf die Mikrostruktur genommen werden. Auf Grundlage des Korngrenzen-Magnetowiderstands [27, 28] kann der Widerstand durch ein äußeres Feld beeinflusst werden. Besonders interessant für die Systeme ist die Möglichkeit der bewussten Modifikation der Korngrenzen beispielsweise durch Dotierung mit einer zweiten, chemischen Phase. 


\section{Ziel dieser Arbeit}

Ziel dieser Arbeit ist die Charakterisierung von Grenzflächen in dünnen $\mathrm{La}_{0,7} \mathrm{Sr}_{0,3} \mathrm{MnO}_{3^{-}}$ Schichten (LSMO) sowie deren bewusste Modifikation durch Hinzufügen einer zweiten, isolierenden Phase unter Ausnutzung einer chemischen Phasenseparation. Weiterhin sollen resultierende physikalische Mechanismen wie der Magnetowiderstand ausgenutzt werden, um Effekte wie beispielsweise das strominduzierte Magnetisierungsschalten in lateral strukturierten Systemen nachzuweisen und gegebenenfalls zu charakterisieren.

Die Möglichkeit der Dotierung von Korngrenzen mit einer zweiten, isolierenden Phase wurde bereits für die nanoskaligen Systeme vielfach nachgewiesen [29, 30,31. Dennoch ist das Transportverhalten nicht vollständig verstanden, was insbesondere die Frage nach einem gestörten Bereich des Manganats an der Grenzfläche zum Isolator betrifft. Aufbauend auf Arbeiten von Köster et al. 29] soll mittels niederfrequenter $1 / f$-Rauschspektroskopie die Grenzfläche LSMO/MgO genauer charakterisiert werden. Zusätzlich soll auch eine Dotierung der Korngrenze mit dem isolierenden $\mathrm{CeO}_{2}$ untersucht werden, da hier interessante geometrische Beziehungen zwischen den Gitterstrukturen vorliegen. Zudem werden strom- bzw. spannungsinduzierte Effekte im undotierten System analysiert.

Die Untersuchungen erfolgen sowohl an nanoskaligen Systemen, also unter Berücksichtigung einer Vielzahl von Grenzflächen, als auch an Bikristallkontakten, an denen gezielt der Einfluss einer einzelnen (dotierten) Korngrenze charakterisiert werden kann. Hierzu werden mittels Bikristall-Substraten erstmalig einzelne, während des Depositionsprozesses dotierte Korngrenzen analysiert.

\section{Aufbau der Arbeit}

In den ersten Kapiteln werden die physikalischen Grundlagen erarbeitet, welche für das Verständnis der erzielten Ergebnisse notwendig sind. Zunächst wird ein Überblick über die Physik der Manganate gegeben, anschließend Magnetowiderstandseffekte vorgestellt und schließlich das theoretische Konzept des Rauschens dargelegt. Im Anschluss werden die verwendeten, experimentellen Techniken dargestellt, wobei ein wesentlicher Schwerpunkt in der Mikrostrukturierung liegt. Die Ergebnisse, welche an den nanoskaligen Systemen und den Bikristallkontakten gewonnen werden, werden in jeweils eigenen Kapiteln behandelt und zunächst einzeln diskutiert. Schließlich werden die in den beiden vorherigen Kapitel gewonnenen Erkenntnisse in einem gemeinsamen Kontext diskutiert, bevor die Ergebnisse zusammengefasst und ein Ausblick gegeben wird. 



\section{Kapitel 2}

\section{Manganate}

Manganate sind bereits seit 1950 durch die Arbeit von Jonker und van Santen bekannt 32]. Die Autoren berichten von einem ferromagnetischen Zustand in gemischten Kristallen aus $\mathrm{LaMnO}_{3}-\mathrm{CaMnO}_{3}, \mathrm{LaMnO}_{3}-\mathrm{SrMnO}_{3}$ und $\mathrm{LaMnO}_{3}-\mathrm{BaMnO}_{3}$. Diese Erkenntnis hat zu weiteren experimentellen und theoretischen Arbeiten, wie zum Beispiel der Entdeckung sowohl ferromagnetischer als auch antiferromagnetischer Phasen in $\mathrm{La}_{1-x} \mathrm{Ca}_{x} \mathrm{MnO}_{3}$ für unterschiedliche Dotierungen $x$ (s.u.) durch Wollan und Koehler [33] oder auch der Beschreibung des ferromagnetischen Zustandes durch den Doppelaustausch durch Zener [34], bzw. Anderson und Hasegawa [35] sowie de Gennes [36], geführt. Anschließend ist das Interesse an der Physik der Manganate vorerst wieder zurückgegangen.

Für die aktuelle Physik sind sie aus mehreren Gründen wieder interessant: Zum einen ist hier der so genannte kolossale Magnetowiderstands-Effekt (CMR) zu nennen. Der CMR beschreibt die Änderung des elektrischen Widerstands in Manganoxiden mit Perowskitstruktur durch ein von außen angelegtes Magnetfeld, wobei die Änderung mehrere Größenordnungen betragen kann. Eine starke Forschungsaktivität auf diesem Gebiet wurde ausgelöst durch die Entdeckung des CMRs in epitaktischen Schichten auch bei Raumtemperatur durch von Helmolt et al. [13], was den CMR zunächst interessant für Anwendungen machte. Allerdings ist der Effekt stark temperaturabhängig und nur im Bereich der kritischen Temperatur des Phasenübergangs besonders ausgeprägt. Zusätzlich werden hohe Magnetfelder im Bereich von 50 kOe benötigt, was insgesamt zu einer Verwerfung der industriellen Anwendung geführt hat. Die physikalischen Mechanismen des CMRs sind hingegen noch immer nicht vollständig verstanden und werden häufig im Zusammenhang mit einer scheinbar ebenfalls intrinsischen Eigenschaft der Manganate, der so genannten elektronischen Phasenseparation, gesehen. Diese beschreibt die Ausbildung zweier elektronisch unterschiedlicher Bereiche (ferromagnetisch-metallisch und paramagnetischisolierend) in chemisch homogenen Proben auch weit unterhalb der kritischen Temperatur des Phasenübergangs [18, 19, 20, 21, 37, 38, 39]. Hierbei sind die mikroskopischen Ursachen weitestgehend unbekannt und Gegenstand aktueller Forschung. Ein Verständnis der elektronischen Phasenseparation könnte dabei nicht nur für die Physik der Manganate relevant sein, sondern auch Einfluss auf die Beschreibung anderer physikalischer Systeme, wie zum Beispiel der Hochtemperatur-Supraleiter, haben [11.

Ein weiterer Punkt, der die Manganate so interessant macht, ist der ferromagnetische 
Grundzustand bei bestimmten Dotierungen (z.B. $\mathrm{La}_{0,7} \mathrm{Sr}_{0,3} \mathrm{MnO}_{3}$ und $\mathrm{La}_{0,7} \mathrm{Ca}_{0,3} \mathrm{MnO}_{3}$ ) und die hohe, aus der komplexen elektronischen Struktur resultierende Spinpolarisation, welche idealerweise bei 100\% liegt [10|. Dabei bezeichnet die Spinpolarisation die Differenz der Ladungsträger mit Spin auf und Spin ab an der Fermikante, normiert auf die Gesamtzahl der Elektronen an der Fermikante (s. Kap. 3.2). Diese Eigenschaft macht die Manganate zu einem idealen Kandidaten als Elektrode in einem Tunnel-MagnetowiderstandsSystem, da der Tunnel-Magnetowiderstands-Effekt stark von der Spinpolarisation des Elektrodenmaterials abhängt.

In diesem Kapitel sollen die physikalischen Grundlagen der Manganate und speziell des Systems $\mathrm{La}_{0,7} \mathrm{Sr}_{0,3} \mathrm{MnO}_{3}$ erläutert werden. Dabei soll zunächst auf die Struktur und anschließend auf die elektronischen Eigenschaften eingegangen werden, wobei aber strukturelle Eigenschaften die elektronischen stark beeinflussen. Da die Physik der Manganate ein weites Feld darstellt, kann kein Anspruch auf Vollständigkeit erhoben werden. Für ausführliche Beschreibungen sei auf Übersichtsartikel in der Literatur verwiesen |12, 40, 16, 17, 41.

\subsection{Struktur der Manganate}

Manganate der Zusammensetzung $\mathrm{AMnO}_{3}$ kristallisieren in Modifikationen der kubischen Perowskitstruktur (s. Abb. 2.1), wobei für diese Arbeit nur die so genannten LanthanManganate $(\mathrm{A}=\mathrm{La})$ verwendet werden. Eine wesentliche Eigenschaft der Perowskitstruktur ist das von einem Sauerstoff-Oktaeder umgebene Mn-Ion.

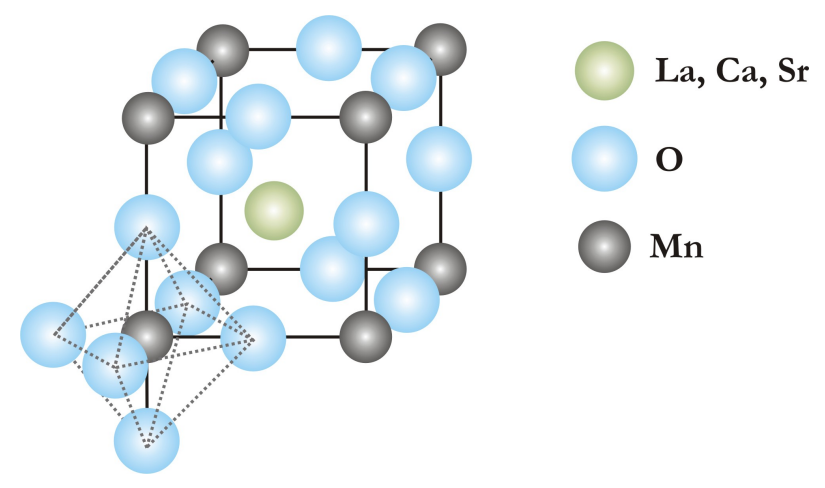

Abbildung 2.1. Quasi-kubische Perowskit-Struktur der Manganate.

Die Modifikationen der kubischen Gitterstruktur stehen in direktem Zusammenhang mit dem $\mathrm{MnO}_{6}$-Oktaeder: Zum einen ist eine Verzerrung desselben aufgrund des JahnTeller-Effekts (siehe 2.2.2), andererseits eine Veränderung der relativen Orientierungen der einzelnen Oktaeder untereinander möglich, woraus eine rhomboedrische bzw. orthorhombische Gitterstruktur resultiert, wie Abbildung 2.2 dargestellt. Hierfür spielt die Gitterpassung einzelner $\mathrm{AO}$ - und $\mathrm{MnO}_{2}$-Ebenen eine wichtige Rolle, welche durch den so genannten Toleranzfaktor $f$ ausgedrückt wird [12]:

$$
f=\frac{1}{\sqrt{2}} \cdot \frac{r_{M n}+r_{O}}{r_{A}+r_{O}}
$$




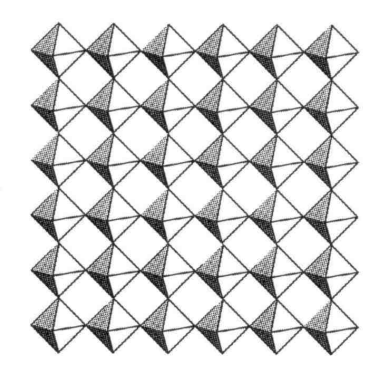

a)
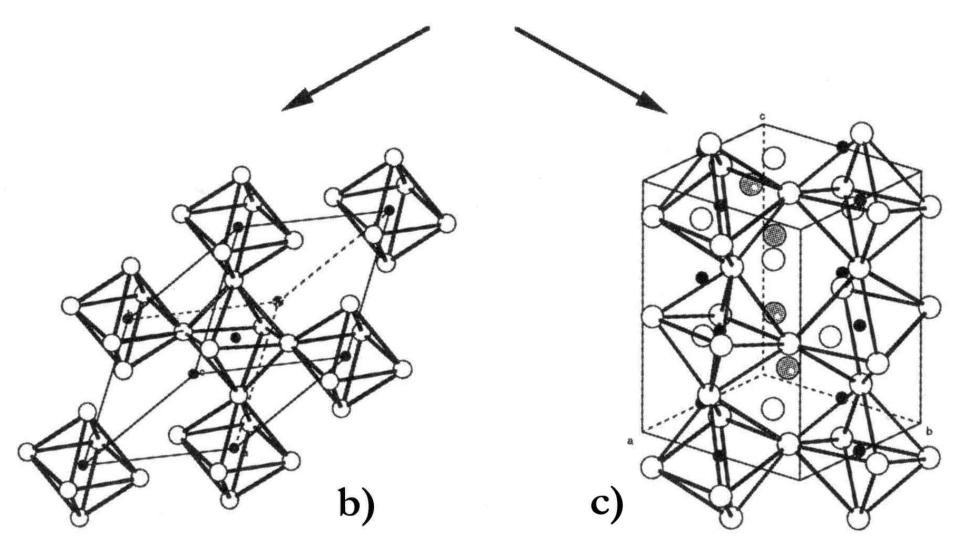

Abbildung 2.2. Modifikationen der Kristallstruktur, welche sich aus dem Toleranzfaktor ergibt. a) kubisch, es liegt keine Verkippung der $\mathrm{MnO}_{6}$-Oktaeder vor. b) Für $0,96<f<1$ ergibt sich eine rhomboedrische Struktur. c) Aufgrund der alternierenden Verkippung der $\mathrm{MnO}_{6}$-Oktaeder bildet sich für $f<0,96$ eine orthorhombische Überstruktur aus. Nach 12 .

Für $f=1$ ergibt sich die kubische Struktur, wohingegen für $0,96<f<1$ die rhomboedrische Struktur und für $f<0,96$ die orthorhombische Struktur stabil ist, in welcher es auch zu Abweichungen vom idealen Bindungswinkel $\left(180^{\circ}\right)$ der Mn-O-Mn-Bindungen kommt (siehe Abb. 2.2). Dieser variiert kontinuierlich mit $f$ und ist wesentlich für die Ausbildung der elektronischen Eigenschaften. Die strukturellen Modifikationen entstehen aufgrund der Verkippungen der $\mathrm{MnO}_{6}$-Oktaeder, wodurch die mittels der unterschiedlichen Ionenradien induzierten Verspannungen reduziert werden können.

Von großer Bedeutung für die Manganate ist die Möglichkeit der so genannten Dotierung. Von besonderem Interesse ist in dieser Arbeit die teilweise Ersetzung des dreiwertigen Lanthans durch ein zweiwertiges Element (Ca, Sr, Ba, ...). Hierdurch wird eine Mischvalenz des Mangans erzeugt, welche immensen Einfluss auf die elektronischen Eigenschaften des Systems hat (siehe 2.2.3). Desweiteren wird aber auch der Toleranzfaktor beeinflusst, da die zweiwertigen Ionen in der Regel einen anderen Radius aufweisen als Lanthan. Zur Berechnung von $f$ wird der gewichtete Mittelwert der Radien herangezogen.

Die entstehenden strukturellen Abweichungen von der idealen kubischen Struktur sind im Allgemeinen aber nur klein, so dass in dieser Arbeit zur Indizierung der Reflexe in den Röntgendiffraktogrammen auf die pseudokubische Gitterstruktur zurückgegriffen wird. 


\section{$2.2 \quad$ Elektronische Struktur}

\subsubsection{Elektronische Struktur von $\mathrm{LaMnO}_{3}$}

Um die elektronische Struktur von $\mathrm{La}_{0,7} \mathrm{Sr}_{0,3} \mathrm{MnO}_{3}$ zu erläutern, soll zunächst auf die Muttersubstanz $\mathrm{LaMnO}_{3}$ näher eingegangen werden (1). Aufgrund der elektronischen Struktur des La-Atoms und der hohen Elektronegativität des Sauerstoffs liegen diese als La ${ }^{3+}$, bzw. $\mathrm{O}^{2-}$-Ionen, also in Edelgaskonfiguration vor. Sie sind deshalb für die Ausbildung elektronischer und magnetischer Phasen nicht relevant. Für das Mn-Ion resultiert demnach eine Wertigkeit von $\mathrm{Mn}^{3+}$, also eine Konfiguration von $[\mathrm{Ar}] 3 d^{4}$. Für das freie Atom besitzen die vier Elektronen in der 3d-Schale aufgrund der Entartung der Orbitale dieselbe Energie. Dieses ändert sich, wenn das $\mathrm{Mn}^{3+}$-Ion in den Festkörper eingebaut ist, sich also in der Mitte des Sauerstoff-Oktaeders befindet. Aufgrund des so genannten Kristallfeldes, welches im wesentlichen durch die negative Ladung der $\mathrm{O}^{2-}$-Ionen hervorgerufen wird, kommt es zur Aufhebung der Entartung der 3d-Orbitale. Dieses wird deutlich, wenn die Orientierung der Mn-3d-Orbitale in Bezug auf die p-Orbitale der Sauerstoffionen gesehen wird (Abb. 2.3): Die Energie eines Elektrons, welches sich in einem Orbital befindet, dass direkt auf ein $\mathrm{O}^{2-}$-Ion zeigt $\left(3 d_{x^{2}-y^{2}}, 3 d_{z^{2}}\right)$, ist aufgrund der Coulomb-Wechselwirkung höher als die eines Elektrons in einem Orbital, das zwischen die Liganden ausgerichtet ist $\left(3 d_{x y}, 3 d_{x z}, 3 d_{y z}\right)$. Demnach haben die Orbitale $3 d_{x y}, 3 d_{x z}$ und $3 d_{y z}$ eine untereinander äquivalente Energie, das Niveau wird als $t_{2 g^{-}}$Orbital bezeichnet. Die Orbitale $3 d_{x^{2}-y^{2}}$ und $3 d_{z^{2}}$ sind ebenfalls energetisch äquivalent, liegen aber höher als das $t_{2 g^{-}}$Orbital und werden mit $e_{g}$ bezeichnet (siehe Abb. 2.3, oben rechts). Der Energieunterschied $\Delta_{\mathrm{CF}}$ zwischen den beiden Niveaus beträgt ca. $\Delta_{\mathrm{CF}} \approx 1 \mathrm{eV}[12]$ und ist bei der Besetzung mit Elektronen $\mathrm{zu}$ vergleichen mit der Stärke der Hundschen Kopplung. Diese beträgt ca. $2-3 \mathrm{eV}|\overline{12}|$ und überwiegt somit. Folglich werden sowohl das $t_{2 g^{-}}$als auch das $e_{g^{-}}$Orbital mit Elektronen parallelem Spins besetzt, bevor sich Elektronen mit antiparallelem Spin in einem Niveau befinden. Im Fall von $\mathrm{Mn}^{3+}$ (insgesamt vier $3 d$-Elektronen) befinden sich also drei Elektronen mit parallelem Spin im $t_{2 g}$-Orbital und ein Elektron, dessen Spin parallel zu denen im $t_{2 g}$-Orbital ausgerichtet ist, im $e_{g}$-Orbital (s. Abb. 2.4. rechts).

\subsubsection{Jahn-Teller-Effekt}

Die Besetzung des $e_{g}$-Orbitals mit einem Elektron führt zu dem so genannten JahnTeller-Effekt. Wiederum bedingt durch die Ausrichtung der Elektronenorbitale kommt es $\mathrm{zu}$ einer Verzerrung des $\mathrm{MnO}_{6}$-Oktaeders. Wie oben erläutert, wird das $e_{g}$-Orbital aus Liganden gebildet, welche direkt auf die $\mathrm{O}^{2-}$-Ionen ausgerichtet sind. Befindet sich das Elektron des $e_{g^{-}}$Orbitals nun im $3 d_{z^{2}}$ Niveau, so kann durch eine Streckung des Oktaeders in z-Richtung, wie sie in Abbildung 2.4, links, angedeutet ist, die Energie dieses Niveaus abgesenkt werden, da sich die Coulomb-Abstoßung verringert. Das unbesetzte

\footnotetext{
${ }^{(1)}$ Das reine $\mathrm{LaMnO}_{3}$ stellt für sich ein komplexes Material dar, dessen Eigenschaften durch Selbstdotierung oder auch Sauerstoffüberschuss stark beeinflusst werden können. Hierauf soll nicht näher eingegangen werden, sondern lediglich die Grundstruktur erläutert werden. Für weiterführende Informationen siehe z.B. 42,43 und Referenzen darin.
} 


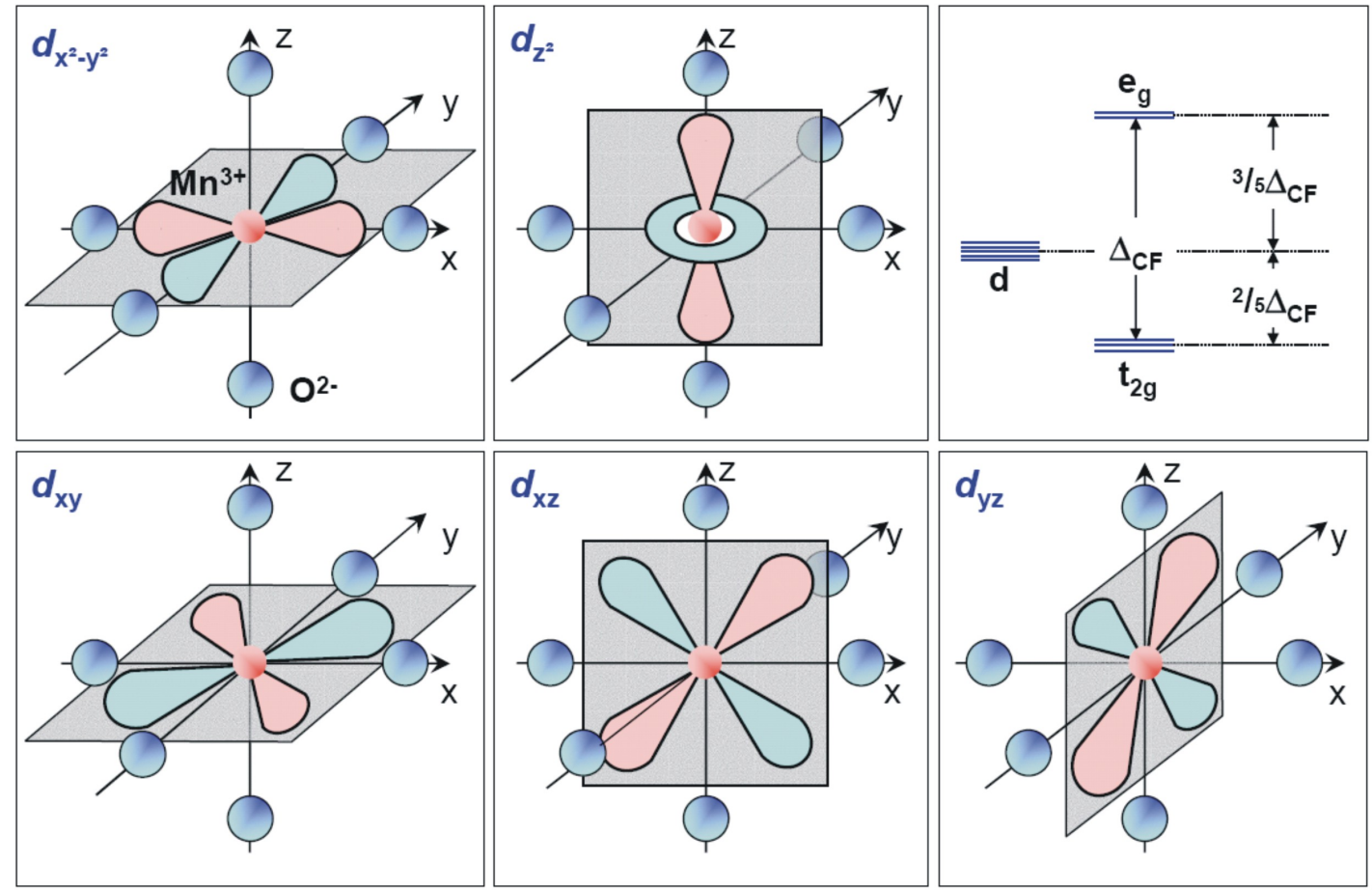

Abbildung 2.3. Bezeichnung und Lage der Mangan 3d-Orbitale im Sauerstoff-Oktaeder. Das Kristallfeld führt zur Aufhebung der Entartung. Vorzeichen der Wellenfunktion: + =rot; $-=$ blau 44$]$.

$3 d_{x^{2}-y^{2}}$ Niveau wird durch diese Verzerrung energetisch angehoben. Auch das $t_{2 g^{-}}$Orbital wird durch den Jahn-Teller-Effekt beeinflusst, die Entartung zwischen den $3 d_{x y^{-}}, 3 d_{x z^{-}}$ und $3 d_{y z}$-Orbitalen wird aufgehoben. Aufgrund der Symmetrie der Verzerrung sind die $3 d_{x z^{-}}$und $3 d_{y z^{-}}$Orbitale energetisch weiterhin gleichberechtigt und werden leicht abgesenkt, wohingegen das $3 d_{x y}$-Orbital durch das Zusammenrücken der $\mathrm{O}^{2-}$-Ionen in der $x y$-Ebene energetisch angehoben wird. Die Gesamtenergie des $t_{2 g}$-Orbitals ändert sich aber nicht 45$]$.

Der hier beschriebene statische Jahn-Teller-Effekt führt zunächst lokal zu einer starken mechanischen Verspannung, welche durch eine geeignete Anordnung aller verspannten Oktaeder zu einer kollektiven Verkippung der $\mathrm{MnO}_{6}$-Oktaeder führt, was wiederum in einer strukturellen Änderung gegenüber der kubischen Struktur resultiert. Sowohl die rhomboedrische als auch die orthorhombische Struktur können realisiert werden. In diesem Sinne ist die Jahn-Teller-Verzerrung als Polaron anzusehen. Ein Polaron bezeichnet dabei allgemein die Kombination von Elektron und seinem Verzerrungsfeld 46].

Aus obiger Argumentation folgt direkt, dass es für ein $\mathrm{Mn}^{4+}$-Ion im Sauerstoffoktaeder zu keiner statischen Jahn-Teller-Verzerrung kommt, da die Gesamtenergieabsenkung ein besetztes $3 d_{z^{2}}$ Niveau voraussetzt. Dieses ist besonders für die dotierten Systeme, welche im Folgenden diskutiert werden sollen, von Bedeutung. Hier liegt eine so genannte Mischvalenz zwischen $\mathrm{Mn}^{3+}$ und $\mathrm{Mn}^{4+}$ vor. 

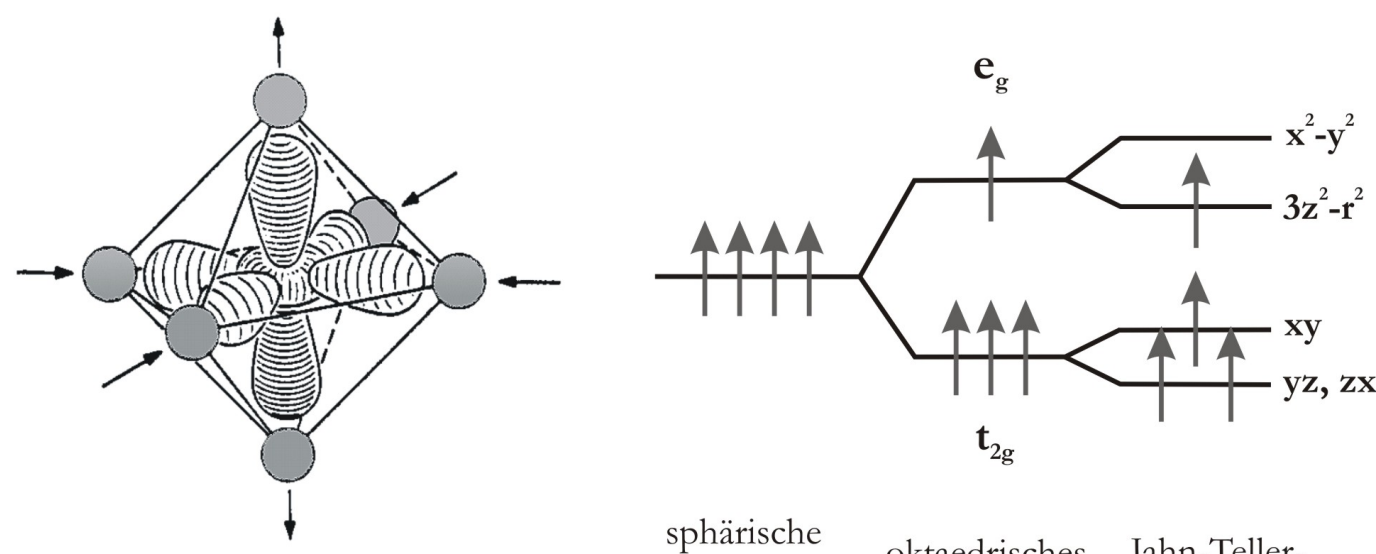

sphärische

Symmetrie (freies Ion)

oktaedrisches Jahn-Teller-

Kristallfeld Effekt

Abbildung 2.4. Aufspaltung der Energieniveaus der 3d-Orbitale durch das Kristallfeld und durch den Jahn-Teller-Effekt (schematisch).

\subsubsection{Austauschmechanismen}

Das reine $\mathrm{LaMnO}_{3}$ ist aufgrund des dominierenden Superaustausches isolierend und ordnet unterhalb der Néel-Temperatur antiferromagnetisch. Wird nun aber das dreiwertige Lanthan teilweise durch ein zweiwertiges Atom ersetzt (hier: Sr), muss aufgrund der Ladungsbilanz das Mangan teilweise als $\mathrm{Mn}^{4+}$-Ion vorliegen. Die stöchiometrische Formel ergibt sich zu

$$
\mathrm{La}_{1-\mathrm{x}}^{3+} \mathrm{Sr}_{\mathrm{x}}^{2+} \mathrm{Mn}_{1-\mathrm{x}}^{3+} \mathrm{Mn}_{\mathrm{x}}^{4+} \mathrm{O}_{3}^{2-}
$$

Diese Mischvalenz zwischen $\mathrm{Mn}^{3+}$ und $\mathrm{Mn}^{4+}$ hat einen enormen Einfluss auf die elektronischen Eigenschaften. So wird z.B. aus dem isolierenden Antiferromagneten $(x=0)$ ein metallischer Ferromagnet ${ }^{(2)}$ für $x \geq 0,18$. Die theoretischen Beschreibungen für diese Mechanismen, der Superaustausch und der Doppelaustausch, sollen im Folgenden qualitativ erläutert werden. Für die quantitative Beschreibung sei auf die Literatur verwiesen $34,35,36,47$.

Zur Aufklärung der magnetischen Struktur wird besonders das Mn-Ion betrachtet, da die $\mathrm{La}^{3+}-, \mathrm{Sr}^{2+}$ - und $\mathrm{O}^{2-}$-Ionen allesamt in Edelgaskonfiguration vorliegen und daher kein magnetisches Moment tragen. Weiterhin ist der Erwartungswert des Bahndrehimpulses für 3d-Übergangsmetalle in kubischer Umgebung gleich Null (,gequencht“). Es ist somit ausreichend, das Spinmoment der Mn-Ionen zu betrachten. Entsprechend ergibt sich aus obigen Überlegungen zur Besetzung der Orbitale ein magnetisches Moment von $S\left(\mathrm{Mn}^{3+}\right)=2$ und $S\left(\mathrm{Mn}^{4+}\right)=\frac{3}{2}$. Zur Leitfähigkeit tragen allerdings nur die Elektronen des $e_{g}$-Orbitals bei, da die Elektronen des $t_{2 g}$-Orbitals durch die Kristallfeldaufspaltung energetisch abgesenkt und so lokalisiert sind.

Sowohl der Super- als auch der Doppelaustausch sind aufgrund des zu großen Abstandes zweier Mn-Ionen indirekt und erfolgen über den Überlapp der Mangan d-Orbitale mit den Sauerstoff p-Orbitalen. Weiterhin bleibt der Spin eines Elektrons beim Ladungstrans-

${ }^{(2)}$ Metallisch wird im Weiteren verwendet, wenn $d \rho / d T>0$, ungeachtet des spezifischen Widerstandes. 
port erhalten, da ein Umklappen desselben energetisch sehr ungünstig ist.

\title{
Superaustausch
}

Zur Beschreibung des Superaustausches, welcher die Wechselwirkung zweier $\mathrm{Mn}^{3+}$-Ionen über ein Sauerstoffion beschreibt, sind zwei Energiebeiträge von wesentlicher Bedeutung. Einerseits ist dies die kinetische Energie, welche durch die Hüpfamplitude $t>0$ charakterisiert wird. Sie ist ein Maß für die Energie, die ein Elektron durch Delokalisierung gewinnt. Andererseits muss die Coulombenergie $U>0$ betrachtet werden, die aufgebracht werden muss, wenn zwei Elektronen denselben Gitterplatz besetzen. Der Superaustausch kann aus einem störungstheoretischen Ansatz zweiter Ordnung berechnet werden [45], woraus allgemein folgt, dass die Kopplungsenergie $J$ proportional ist zum Quadrat der Hüpfamplitude $t$ geteilt durch die für einen angeregten Zustand nötige Energie $U$ [45]:

$$
J \propto-\frac{t^{2}}{U}
$$

In der qualitativen Beschreibung des Superaustausches (Abb. 2.5) wird das Sauerstoffion vernachlässigt. Dargestellt sind zwei benachbarte Mn-Ionen mit jeweils zueinander ausgerichteten $3 d_{z^{2}}$-Orbitalen. Aufgrund der Jahn-Teller-Verzerrung ist die Entartung des $t_{2 g}$-Orbitals aufgehoben, s.d. nur ein Niveau zur Verfügung steht, welches von zwei Elektronen mit antiparallelem Spin besetzt werden kann. Für den Fall a) verhindert das PauliPrinzip den Elektronentransport, der Energiegewinn ist Null. Für den Fall b) kommt es zu einer Nettoenergieabsenkung durch den erlaubten Hüpfprozess. Dieser Prozess ist gleichzeitig mit einer antiferromagnetischen Kopplung verbunden, da die Spinrichtung eines einzelnen Elektrons im $t_{2 g}$-Orbital aufgrund der starken Hundschen Kopplung parallel zu der Spinrichtung der Rumpfelektronen im $e_{g}$-Orbital ist.

a)
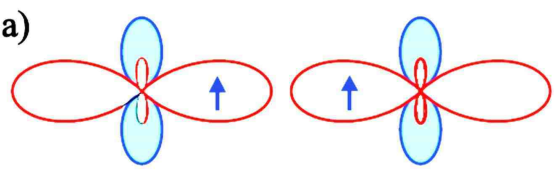

$\Delta E=0$

b)

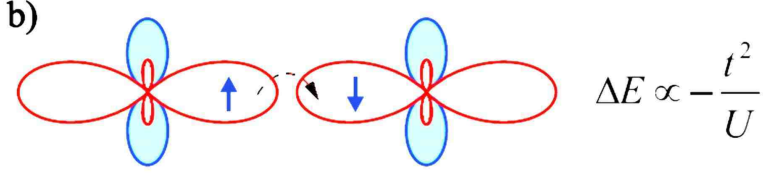

\begin{abstract}
Abbildung 2.5. Schema zum Ein-Niveau-Superaustausch am Beispiel zweier zueinander gerichteter $\mathrm{d}_{z^{2}}$-Orbitale: Die antiparallele Spinorientierung (b) wird energetisch bevorzugt [44.
\end{abstract}

Die obigen Überlegungen zum Superaustausch machen zwar eine antiferromagnetische Kopplung plausibel, greifen aber zur vollständigen Aufklärung des Mechanismuses zu kurz. Eine deutlich bessere Beschreibung gelingt durch die so genannten Goodenough-KanamoriAnderson-Regeln (GKA) [48,49,50], welche insbesondere die orbitale Struktur in Betracht ziehen. Durch das Anwenden der GKA-Regeln, welche hier nicht weiter erläutert werden 
sollen, kann unter Annahme einer bestimmten orbitalen Struktur leicht die magnetische Struktur der beiden Grenzfälle $\mathrm{LaMnO}_{3}$ und $\mathrm{SrMnO}_{3}$ erklärt werden (s. z.B. [50|). Für $\mathrm{LaMnO}_{3}$ ergibt sich eine ferromagetische Kopplung innerhalb der $a$ - $b$-Ebene und eine antiferromagnetische Kopplung in $c$-Richtung. Diese Art von antiferromagnetischer Ordnung wird auch als A-Typ bezeichnet. Für $\mathrm{SrMnO}_{3}$ hingegen koppelt jedes Mn-Ion mit allen Nachbarn antiferromagnetisch, es bildet sich die so genannte Typ-G Struktur.

\section{Doppelaustausch}

Das Einbringen von Löchern in die $e_{g}$-Orbitale durch das teilweise Ersetzen des Lanthans durch zweiwertige Elemente lässt einen weiteren Wechselwirkungsmechanismus, den Doppelaustausch, auftreten. Die hervorgerufenen Änderungen wie zum Beispiel das Auftreten eines metallischen, ferromagnetischen Zustandes lassen sich dadurch erklären. Auch ein qualitatives Verständnis des CMRs kann gegeben werden.

(a)

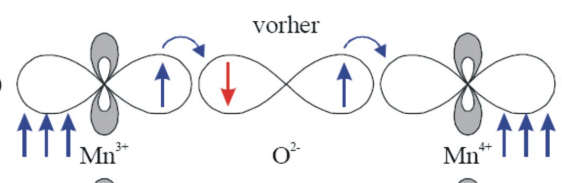

(b) $\uparrow \uparrow \uparrow$

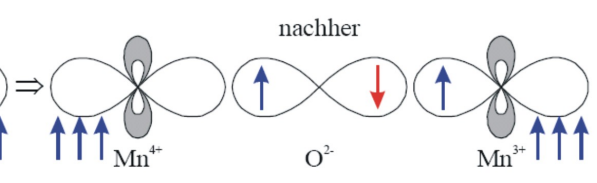

Abbildung 2.6. Schematische Darstellung des Doppelaustausches. Nach [50].

Der Doppelaustausch soll wieder an einer Schemazeichnung plausibel gemacht werden (Abb. 2.6). Die Situation erscheint der des Superaustausches ähnlich, allerdings findet der Doppelaustausch zwischen $\mathrm{Mn}^{3+}$ - und $\mathrm{Mn}^{4+}$-Ionen statt. Diese Tatsache hat einen wesentlichen Einfluss auf die Wechselwirkung, da für den Hüpfprozess eines Elektrons aus dem $e_{g}$-Orbital des $\mathrm{Mn}^{3+}-$ Ions in das unbesetzte $e_{g}$-Orbital des $\mathrm{Mn}^{4+}-$ Ions die abstoßende Coulombwechselwirkung entfällt. Für diese Art Hüpfprozess müssen nun wieder alle Energieterme betrachtet werden. Zunächst sei der Fall (a) betrachtet: Die Rumpfspins der beiden beteiligten Mn-Ionen sind parallel ausgerichtet, der Spin des Elektronen im $e_{g}$-Orbital des $\mathrm{Mn}^{3+}-$ Ions aufgrund der Hundschen Regel ebenfalls. Dieses Elektron kann sich ohne Aufbringen der Hundschen Kopplungsenergie zum $\mathrm{Mn}^{4+}$-Ion bewegen. Es kann also durch Delokalisierung seine Gesamtenergie verringern. Im Fall (b) hingegen stehen die Rumpfspins antiparallel. Bewegte sich das Elektron nun ohne Änderung seines Spins zum $\mathrm{Mn}^{4+}$-Ion, so müsste es entgegen der Hundschen Kopplungsenergie das $e_{g}$-Orbital besetzten. So stünde dem Gewinn an kinetischer Energie der Aufwand der Hundschen Kopplungsenergie gegenüber, s.d. der Fall (a) energetisch deutlich günstiger ist. Hieraus ist sofort ersichtlich, dass eine ferromagnetische Ordnung energetisch bevorzugt wird, welche auch gleichzeitig mit einer guten elektrischen Leitfähigkeit, also einem metallischen Zustand verknüpft ist. Die $e_{g}$-Elektronen bilden dabei ein Band, dessen Breite $W$ im Rahmen einer einfachen tight-binding-Näherung proportional zu $t$ ist 46. Andererseits wird die Bandbreite auch durch andere Faktoren wie hydrostatischen Druck beeinflusst. 
Weiterhin ergibt sich aus dem einfachen Modell des Doppelaustausches eine wichtige Konsequenz für die Leitungselektronen. Aufgrund der Hundschen Kopplung, welche letztlich für den Doppelaustausch verantwortlich ist, ergibt sich, dass im Grundzustand die Spins aller Leitungelektronen parallel ausgerichtet sind. Diese 100\%ige Spinpolarisation wurde mittels spinpolarisierter Photoemissions-Spektroskopie experimentell bestätigt [10 und ist von enormer Bedeutung für das spinabhängige Tunneln (s. Kap. 3.2).

Werden nun thermische Anregungen in diesem Modell zugelassen, so muss nach dem effektiven Hüpfmatrixelement $t_{\text {eff }}$ gefragt werden. Nach obiger Beschreibung des Doppelaustausches ist direkt einsichtigt, dass dieses mit dem Winkel $\theta_{i j}$ zwischen den Rumpfspins zweier benachbarter Mn-Ionen verknüpft ist:

$$
t_{e f f}=t \cdot \cos \frac{\theta_{i j}}{2}
$$

Hieraus folgt eine maximale Hüpfamplitude und somit Leitfähigkeit für eine parallele Orientierung und eine verschwindende für antiparallele Orientierung. Für hohe Temperaturen kommt es aufgrund der thermischen Anregungen zu einer statistischen Ausrichtung der Spins, s.d. sowohl der Ferromagnetismus, als auch gleichzeitig die elektrische Leitfähigkeit unterdrückt wird. Es findet ein Metall-Isolator-Übergang (MIT, metal-insulator transition) an der Curie-Temperatur statt. In diesem Zusammenhang kann auch eine einfache Erklärung des CMRs gegeben werden: Ein äußeres Magnetfeld richtet die ungeordneten Spins aus und ermöglicht so eine erhöhte Leitfähigkeit. Da für tiefe Temperaturen die Spinordnung durch den Doppelaustausch gegeben ist, ist der Effekt hier klein. In der Nähe der Curie-Temperatur hingegen ist die Kopplung nur schwach, s.d. nur relativ kleine Energien notwendig sind, um die Spinordnung wieder herzustellen ${ }^{(3)}$. Für höhere Temperaturen reicht die Energie des äußeren Feldes nicht aus, um die thermische Anregung auszugleichen. Infolgedessen ist der Effekt um den Phasenübergang herum am größten. Es sei darauf hingewiesen, dass diese Erklärung für den CMR zu kurz greift und den Effekt nicht vollständig beschreibt. Auch die oben gegebene Erklärung des Doppelaustausches ist nur schematisch und beschreibt die Kopplung unzureichend. Auf die Grenzen des Modells wird unten eingegangen.

\subsubsection{Das Phasendiagramm $\mathrm{La}_{1-x} \mathrm{Sr}_{x} \mathrm{MnO}_{3}$}

Im Folgenden soll das Phasendiagramm für $\mathrm{La}_{1-x} \mathrm{Sr}_{x} \mathrm{MnO}_{3}$, welches in Abbildung 2.7 dargestellt ist, diskutiert werden. Für $x=0$ ergibt sich die bereits bekannte antiferromagnetische, isolierende Phase des $\mathrm{LaMnO}_{3}$, welche vollständig mit dem Superaustausch erklärt werden kann. Die Néeltemperatur des orthorhombischen Kristalls beträgt dabei $T_{N} \approx 140 \mathrm{~K}$. Durch Dotierung mit Strontium ändert sich die magnetische Ordnung für $x>0,1$, es stellt sich eine ferromagnetische Ordnung ein, welche aber erst für $x>0,175$ metallisch wird. Innerhalb des ferromagnetischen, metallischen Bereichs tritt ein struktu-

\footnotetext{
${ }^{(3)}$ Auch wenn nur kleine Energien notwendig sind, so bedeutet dies für den allgemeinen Laborbetrieb hohe Magnetfelder in der Gößenordnung von $50 \mathrm{kOe}$, da typische Laborfelder klein sind gegenüber internen Magnetfeldern in magnetisch geordneten Festkörpern.
} 


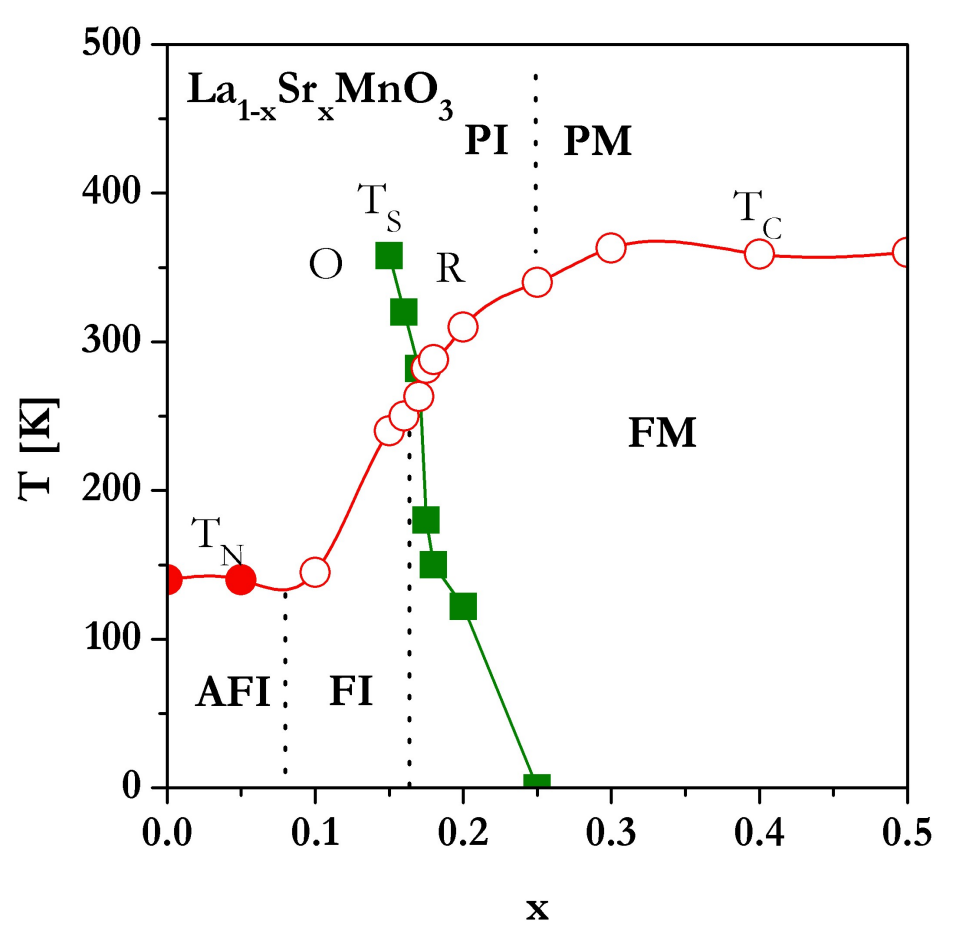

\begin{abstract}
Abbildung 2.7. Elektronisches, magnetisches und strukturelles Phasendiagramm von $\mathrm{La}_{1-x} \mathrm{Sr}_{x} \mathrm{MnO}_{3}$. Kreise bezeichnen die Néel-Temperatur ( $\mathrm{T}_{\mathrm{N}}$, geschlossene Symbole), bzw. die Curie-Temperatur ( $\mathrm{T}_{\mathrm{C}}$, offene Symbole). Die geschlossenen Quadrate kennzeichnen den strukturellen Phasenübergang $\left(T_{S}\right)$ zwischen der rhomboedrischen $(R)$ Hochtemperaturphase und der orthorhombischen $(\mathrm{O})$ Tieftemperaturphase. Die weiteren Abkürzungen stehen für paramagnetisch isolierend (PI), paramagnetisch metallisch (PM), antiferromagnetisch isolierend (AFI), ferromagnetisch isolierend (FI) und ferromagnetisch metallisch (FM). Nach 12 .
\end{abstract}

reller Phasenübergang zur rhomboedrischen Phase auf, der im Grundzustand durch die Änderung des Toleranzfaktors getrieben wird. Weiterhin nimmt die Curie-Temperatur für $x \leq 0,33$ mit zunehmenden $x$ weiter zu $\left(T_{C}(x=0,3) \approx 370 \mathrm{~K}\right)$. Einerseits ist dieses auf die zunehmende Dominanz des Doppelaustausches über den Superaustausch zurückzuführen, da mehr $\mathrm{Mn}^{4+}$-Ionen gebildet werden, andererseits ist hierfür der sich ändernde Toleranzfaktor verantwortlich, der durch das Einbringen des im Gegensatz zum La ${ }^{3+}$ größeren $\mathrm{Sr}^{2+}$ näher an das ideale Verhältnis von 1 heranrückt und so den Überlapp der beteiligten Orbitale optimiert.

Ungeachtet der Tatsache, dass das Phasendiagramm mittels der Konkurrenz der beiden Austauschmechanismen und des Toleranzfaktors in großen Teilen verstanden werden kann, ergeben sich noch immer Schwierigkeit im Verständnis vieler physikalischer Eigenschaften. So ist zum Beispiel weitgehend unverstanden, warum der CMR genau für die Dotierung den höchsten Wert erreicht, bei der gerade noch ein metallischer, ferromagnetischer Zustand vorhanden ist [17]. Aus diesem Grunde werden die mit dem dominierenden Doppelaustausch konkurrierenden Prozesse in der aktuellen Forschung als mindestens ebenso wichtig für die Erklärung des CMR angesehen [52,16].

Das experimentell bestimmte Phasendiagramm zeigt die Schwächen der beiden oben 
vorgestellten Kopplungsmechanismen deutlich auf. So können die Bereiche, in denen das Material ferromagnetisch und isolierend oder auch antiferromagnetisch und metallisch $(x>0,5)$ ist, nicht mittels des Doppelaustausches erklärt werden. Auch für den paramagnetischen Bereich treten sowohl metallische als auch isolierende Dotierungsbereiche auf. Weiterhin tritt in $\mathrm{La}_{0,8} \mathrm{Ca}_{0,2} \mathrm{MnO}_{3+y}$ eine Verschiebung der Curie-Temperatur um $20 \mathrm{~K}$ auf, wenn das Sauerstoffisotop $\mathrm{O}^{18}$ verwendet wird [53. Ein Doppelaustausch-Modell in der oben vorgestellten Form ist demnach nicht anwendbar. Zwei wesentliche Aspekte werden in der aktuellen Forschung diskutiert, um die Diskrepanzen zu verstehen. Einerseits wird versucht, die im Doppelaustausch vernachlässigte Jahn-Teller-Verzerrung in das Modell zu integrieren, also einen polaronischen Transport zu etablieren. Andererseits wird die Relevanz der zum dominierenden Prozess konkurrierenden Phase betont. Dieses Modell der elektronischen Phasenseparation soll im folgenden Kapitel kurz vorgestellt werden.

\subsection{Elektronische Phasenseparation}

Besonders die (quantitative) Erklärung des CMRs in einem elektronisch homogenen Material erscheint nicht möglich. So müssen zum Beispiel CMR-Szenarien, welche auf dem Doppelaustausch oder auf Anderson-Lokalisierung beruhen, verworfen werden, ebenso wie Theorien, welche auf einem Bild basieren, in dem der paramagnetisch isolierende Zustand aus kleinen Polaronen besteht 52 .

Einen weiteren Erklärungsansatz bietet das Konzept der elektronischen Phasenseparation in Manganaten, welches das Auftreten zweier elektronisch unterschiedlicher Bereiche in einer chemisch homogenen Probe beschreibt (s. Abb. 2.8). So können metallische, ferromagnetische Cluster mit antiferromagnetischen, isolierenden Clustern auf der Nanometerskala koexistieren. Dieses Modell ermöglicht insbesondere ein tieferes Verständnis der Phasendiagramme und des CMRs [54 und ist zudem mit direkten experimentellen Beobachtungen in Einklang zu bringen $[18,19,20,21$. So wurden durch rastersondenmikroskopische Methoden auch für Temperaturen weit unterhalb der Phasenübergangstemperatur Bereiche stark unterschiedlicher Leitfähigkeit gefunden, deren Anteile durch Temperatur und äußeres Magnetfeld beeinflusst werden können. Das Anlegen eines Magnetfeldes zum Beispiel führt zu einem höheren Anteil der ferromagnetischen, metallischen Phase, was eine Erhöhung der Leitfähigkeit zur Folge hat. Eine Erhöhung der Temperatur hingegen resultiert in einer Verringerung des Anteils, die Leitfähigkeit sinkt. Dabei wird die Leitfähigkeit durch einen perkolativen Prozess modelliert, wodurch insbesondere der Metall-Isolator-Übergang einsichtig wird. Die großen Erfolge des Modells liegen darin, auch quantitativ eine gute Vorhersage zu liefern.

Die elektronische Phasenseparation spielt in der momentanen Diskussion der Manganate ein herausragende Rolle. Ein ausführlicher Überblick über die elektronische Phasenseparation ist in [16] gegeben, eine kurze Einführung in |55|. Das Konzept kann möglicherweise auch zur Klärung weiterer Phänomene in anderen stark korrellierten Systemen wie Hochtemperatur-Supraleitern beitragen [52]. 


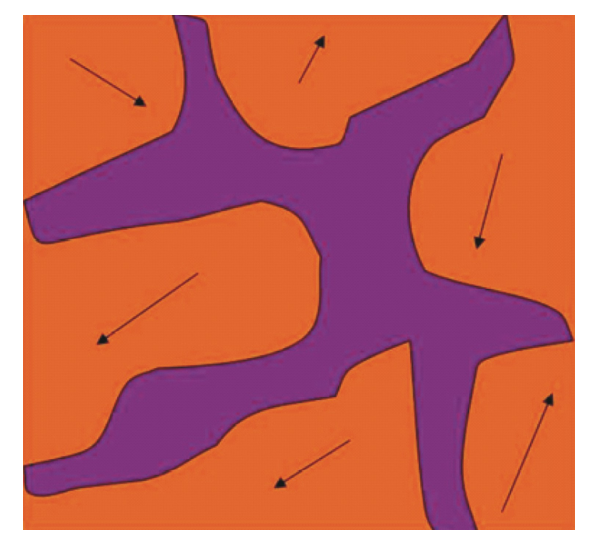

Abbildung 2.8. Schematische Darstellung der elektronischen Phasenseparation in Manganaten mit ferromagnetischen Inseln (orangefarben), deren Magnetisierungen statistisch verteilt sind, getrennt durch die konkurrierende, isoliernde Phase (fliederfarben), welche in einem äußeren magnetischen Feld schmilzt 52 .

\subsection{Einfluss von Polaronen}

Als Polaron wird allgemein die Kombination eines Elektrons mit dessen Verzerrungsfeld bezeichnet [46]. Bisher wurden Polaronen im metallischen Zustand der Manganate vernachlässigt. Aufgrund der Delokalisierung der $e_{g}$-Elektronen und der gemischten Valenz der Mn-Ionen wurde angenommen, dass die Jahn-Teller-Verzerrung im Grundzustand oberhalb einer bestimmten Dotierung verschwindet [12]. In der isolierenden Phase $\left(T>T_{C}\right)$ hingegen kommt es zur Lokalisierung der Ladungsträger, s.d. auch die JahnTeller-Verzerrung aufgrund ihrer hohen Wechselwirkungsenergie von ca. $1 \mathrm{eV}$ wieder auftritt. Dieser Effekt ist besonders für Systeme mit schmalen Ein-Elektronen Bandbreiten wie $\mathrm{La}_{1-x} \mathrm{Ca}_{x} \mathrm{MnO}_{3}$ und $\mathrm{Pr}_{1-x} \mathrm{Ca}_{x} \mathrm{MnO}_{3}$ von Bedeutung, in denen der polaronische Transport oberhalb der Übergangstemperatur nachgewiesen werden konnte 51 .

Wie kürzlich durchgeführte Experimente gezeigt haben, ist die Vernachlässigung der Polaronen im metallischen Zustand unzulänglich. So haben Sudheendra et al. 23] direkte Hinweise auf die Existenz von korrelierten Polaronen im metallischen Zustand einer chemisch geordneten $\mathrm{La}_{3 / 4} \mathrm{Ca}_{1 / 4} \mathrm{MnO}_{3}$-Probe gefunden. Solche Proben sind aufgrund der Kationen-Ordnung bis auf atomarer Skala frei von elastischen Spannungen und somit elektronisch homogen. Dennoch zeigen sie einen großen CMR-Effekt [56]. Auch Mathieu et al. beobachten einen CMR ohne Anzeichen einer elektronischen Phasenseparation [57. Folglich greift das Konzept der elektronischen Phasenseparation zur Erklärung des auftredenden CMRs in diesen Fällen nicht. Ein Hinweis auf den polaronische Einfluss auf den CMR ist die unterschiedliche Orientierung der von Sudheendra et al. beobachteten Ladungs-Dichte-Welle oberhalb und unterhalb der Curie-Temperatur [23.

Die Experimente zeigen, dass polaronische Effekte nicht nur in isolierenden Phasen wie beispielsweise $\operatorname{Pr}_{1-x} \mathrm{Ca}_{x} \mathrm{MnO}_{3}$ eine wichtige Rolle spielen [22], sondern auch in der metallischen Phase der Manganate bedeutsam sind. Ein ausführlicher Überblick über den Einfluss der Polaronen auf die Physik der Manganate auch im Hinblick auf andere Konzepte wie die elektronische Phasenseparation ist in [24] gegeben. 


\subsection{Extrinsische Effekte}

\subsubsection{Dünne Schichten}

Die Physik der Manganate bietet durch die vergleichbaren Energieskalen der auftretenden konkurrierenden Prozesse eine Vielzahl von zum Teil noch unverstandenen, offensichtlich intrinsischen Eigenschaften. Neben diesen intrinsischen Effekten bietet sich aber auch die Möglichkeit, das System von außen zu beeinflussen, also extrinsische Effekte zu erzeugen. Hierauf soll im Folgenden kurz eingegangen werden.

Von großer Bedeutung für die aktuelle Physik sind dünne Schichten, also Proben, deren Ausdehnung zumindest in einer Dimension stark eingeschränkt ist ${ }^{(4)}$. Für solche Systeme sind Grenz- und Oberflächeneffekte besonders ausgeprägt, da das Verhältnis von Volumen zu Oberfläche deutlich geändert wird. Die Wahl der Substrate ist dabei ebenso von großer Bedeutung, da sowohl Wachstumsrichtung und Wachstumsmodus als auch der Gitterparameter maßgeblich beeinflusst werden können. Diese Einflüsse erlauben eine bewusste Kontrolle der Probeneigenschaften, da z.B. durch den Gitterparameter der orbitale Überlapp und infolgedessen die MIT-Temperatur geändert wird. Dieses soll im Folgenden an einem Beispiel verdeutlicht werden, siehe auch [58,59]. Häufig verwendete Substrate zur Deposition von Manganaten sind $\mathrm{SrTiO}_{3}$ (STO, kubisch, $a=3,91 \AA$ ), $\mathrm{LaAlO}_{3}$ (LAO, pseudokubisch, $a=3,82 \AA$ ) und $\mathrm{MgO}$ (kubisch, $a=4,20 \AA$ ), diskutiert werden werden soll der Fall von $\mathrm{La}_{0,7} \mathrm{Ca}_{0,3} \mathrm{MnO}_{3}$ (LCMO) mit einem Volumengitterparameter von $a=3,86 \AA$. In allen Fällen kommt es zu einem (001)-orientierten Wachstum. Die Fehlanpassung von 1,2\% auf dem STO-Substrat resultiert in einer tetragonalen Struktur. Die Schicht steht unter Zugspannung, d.h. der Gitterparameter ist in c-Richtung kleiner als in der a-b-Ebene. Diese Verspannung bleibt auch für vergleichsweise große Schichtdicken (bis zu $80 \mathrm{~nm}$ ) erhalten und führt zu einer drastischen Abnahme der Phasenübergangstemperatur. Diese Abnahme ist auch für Schichten auf LAO zu beobachten, welche unter Druckspannung stehen (Gitterfehlpassung -1,0\%). Allerdings werden in diesem Fall die Spannungen mit steigender Schichtdicke abgebaut. Anders verhält es sich für $\mathrm{MgO}$, wo die Fehlpassung sehr hoch ist (9,1\%). In einem Bereich nahe der Grenzfläche zum Substrat (ca. $2 \mathrm{~nm}$ ) treten Versetzungen auf, wodurch die hohe mechanische Spannung abgebaut wird. Nach dem Abbau der Spannungen wächst die Probe annähernd defektfrei und unverspannt auf, der Gitterparameter liegt nahe des Volumenwertes. Diese Schichten zeigen die höchsten Übergangstemperaturen, wobei auch die Breite des Übergangs deutlich schmaler ist, verglichen mit denen verspannter Proben. Wird die Schichtdicke aber soweit verringert, dass der Bereich des Spannungsabbaus maßgeblich wird, so wird ein deutlicher Einfluss der Versetzungen deutlich [60].

Unter Verwendung anderer Substrate können auch weitere Wachstumsmodi erzwungen werden. Für diese Arbeit ist dabei das kolumnare Wachstum von LSMO auf (0001)orientiertem $\mathrm{Al}_{2} \mathrm{O}_{3}$ von besonderer Bedeutung. Die kolumnaren Strukturen haben dabei

\footnotetext{
(4) Üblicherweise bezieht sich der Begriff dünne Schicht auf Proben, die mit bestimmten Verfahren hergestellt werden. Die eigentliche Definition ist daher unabhängig von der Dicke der Probe, welche gelegentlich die Dimension konventionell hergestellter Präparate überschreiten kann.
} 
a)

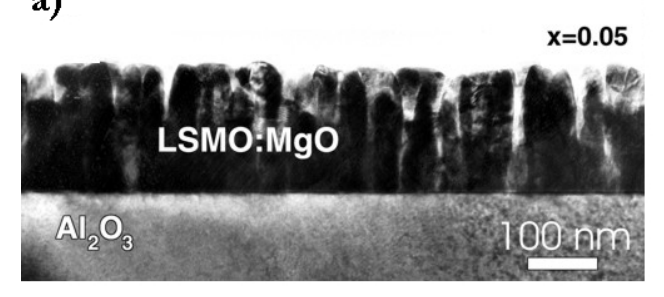

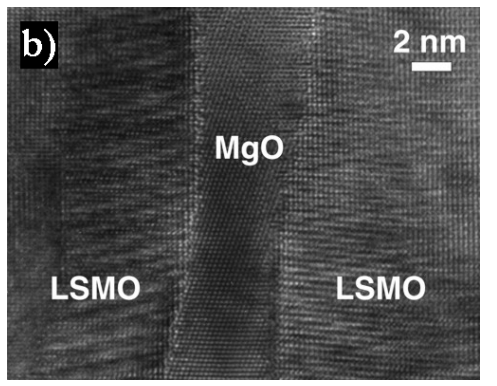

Abbildung 2.9. Querschnittsaufnahmen mittels Transmissions-Elektronen-Mikroskopie von $(\mathrm{LSMO})_{0,5}:(\mathrm{MgO})_{0,5}[29]$.

zumeist eine (111)-Orientierung in c-Richtung, sind aber in der a-b-Ebene statistisch gegeneinander gedreht. Dadurch entstehen Korngrenzen, die wiederum maßgeblichen Einfluss auf die elektronischen Eigenschaften des Systems haben (s. Kap. 3.4).

\subsubsection{Dotierung von Korngrenzen in $\mathrm{La}_{0,7} \mathrm{Sr}_{0,3} \mathrm{MnO}_{3}$}

Eine weitere Möglichkeit zur Beeinflussung der physikalischen Eigenschaften der Manganate bietet sich durch das Hinzusetzen weiterer chemischer Phasen. Eine Voraussetzung hierfür ist die Stabilität des Manganats unter Hinzufügen der zweiten Phase, also eine chemische Phasenseparation. Aus Gründen, welche im Kapitel 3.4 erläutert werden sollen, werden als zweite Phase Isolatoren verwendet, wobei die Liste der bisher in Kombination mit LSMO und LCMO untersuchten Materialien lang ist: MgO 61, $\mathrm{SrTiO}_{3}$ [62], $\mathrm{CoFe}_{2} \mathrm{O}_{4}$ [63, $\mathrm{Al}_{2} \mathrm{O}_{3}$ [64, 65], diverse Gläser [66, 67], $\mathrm{Pr}_{0.5} \mathrm{Sr}_{0.5} \mathrm{MnO}_{3}$ |68], $\mathrm{BaFe}_{11.3}(\mathrm{ZnSn})_{0.7} \mathrm{O}_{19}$ [69], Polymere [70], $\mathrm{ZrO}_{2}$ [71], $\mathrm{ZnO}$ [72, 73], $\mathrm{TiO}_{2}$ |74] oder auch $\mathrm{LuMnO}_{3}|75|$.

Für diese Arbeit sind die beiden Systeme LSMO:MgO [29,76] und LSMO:CeO $\mathrm{C}_{2} 77$, 30.78 von besonderer Bedeutung, welche kurz vorgestellt werden. Beide Isolatoren bieten Vorteile gegenüber den oben erwähnten Systemen. In Hinblick auf eine Untersuchung der magnetoresistiven Eigenschaften des Systems (spinpolarisiertes Tunneln, siehe 3.4) wird $\mathrm{MgO}$ (NaCl-Struktur, $a=4,20 \AA, \mathrm{E}_{g}=7,8 \mathrm{eV}|79|$ ) allgemein als ein vielversprechendes Material angesehen, was hauptsächlich auf die (komplexe) Bandstruktur zurückgeführt werden kann. Zusätzlich konnte durch Köster et al. die chemische Phasenseparation in dünnen Schichten gezeigt und gezielt gesteuert werden 29,76. Unter Verwendung eines (0001)-orientierten $\mathrm{Al}_{2} \mathrm{O}_{3}$-Substrats kommt es zu kolumnarem Wachstum, wobei das $\mathrm{MgO}$ einzelne LSMO-Säulen trennt, siehe Abbildung 2.9 a). Dabei kommt es zur Ausbildung scharfer Grenzflächen, Abbildung 2.9 b). Das MgO ist also in den Korngrenzen des LSMOs lokalisiert, weshalb auch von Dotierung der Korngrenzen gesprochen wird. Dieses ist nicht $\mathrm{zu}$ verwechseln mit der in 2.2 .3 besprochenen Dotierung.

Das zweite System, LSMO:CeO ${ }_{2}$, ist besonders in struktureller Hinsicht interessant. So war die chemische Phasenseparation lange umstritten, da auch versucht wurde, so genanntes elektronen-dotiertes Manganat zu präparieren, $\mathrm{La}_{0.67} \mathrm{Ce}_{0.33} \mathrm{MnO}_{3}$. Statt einer Lochdo- 
tierung mittels zweiwertiger Atome wird durch ein vierwertiges Atom wie Cer theoretisch eine Elektronendotierung erreicht 5 , Letztlich aber hat eine Arbeit von Stingl gezeigt, dass eine Präparation dünner $\mathrm{La}_{0.67} \mathrm{Ce}_{0.33} \mathrm{MnO}_{3}$-Schichten mittels Sputterdeposition und Metallorganischen-Aerosol-Deposition nicht möglich ist und es zur Ausscheidung einer Ceroxid-reichen Phase kommt 77]. Diese Tatsache lässt sich positiv in eine Bestätigung der Phasenseparation LSMO: $\mathrm{CeO}_{2}$ umkehren. Dabei zeigt sich eine interessante strukturelle Beziehung zwischen LSMO und $\mathrm{CeO}_{2}$ : Die $\mathrm{CaF}_{2}$-Struktur ${ }^{(6)}$ hat mit $a=5,41 \AA$ einen um den Faktor 0,986 $\sqrt{2}$ größeren Gitterparameter als LSMO. Unter einer $45^{\circ}$-Rotation ergibt sich also eine sehr geringe Gitterfehlpassung der beiden Materialien. Weiterhin ist die Bandlücke mit $\mathrm{E}_{g}=3,20 \mathrm{eV}$ |81| deutlich geringer verglichen mit $\mathrm{MgO}$, wodurch der Gesamtwiderstand sinkt.

\subsection{Widerstandsschalten in Perowskiten}

Neben dem bereits vorgestelltem LSMO ist $\mathrm{Pr}_{0.7} \mathrm{Ca}_{0.3} \mathrm{MnO}_{3}$ (PCMO) in der aktuellen Forschung von besonderem Interesse, nicht zuletzt aufgrund der Tatsache, dass der elektrische Widerstand nicht nur durch ein äußeres Magnetfeld beeinflusst werden kann, sondern auch durch eine elektrische Spannung bzw. einen Strom. PCMO ist im Grundzustand aufgrund der kleinen Bandbreite der $e_{g}$-Elektronen ein ladungsgeordneter, antiferromagnetischer Isolator, dessen Néel-Temperatur $\mathrm{T}_{N}=170 \mathrm{~K}$ kleiner ist als die kritische Temperatur der Ladungsordnung, $\mathrm{T}_{\mathrm{CO}} \approx 230 \mathrm{~K}[12]$. Ein äußeres Magnetfeld bewirkt das Schmelzen der ladungsgeordneten Phase und damit einen Übergang in eine ferromagnetische, metallische Phase 12. Die hiermit verbundenen Widerstandsänderungen erreichen mehrere Größenordnungen. Diese enorme Widerstandsverringerung kann aber auch in Abwesenheit eines magnetischen Feldes erreicht werden: So konnten Asamitsu et al. durch Änderung der angelegten Spannung eine Widerstandsänderung von mehr als vier Größenordnungen nachweisen, welche ebenfalls auf einen Übergang der ladungsgeordneten, isolierenden Phase in eine ferromagnetische, metallische Phase zurückgeführt wird [14. Dabei ist die Ursache (elektrisches Feld oder Strom) des so genannten kolossalen Elektrowiderstandes (engl.: colossal electroresistance, CER) Gegenstand kontroverser Diskussionen.

Der CER ist aufgrund der Beteiligung der ladungsgeordneten Phase auf Temperaturen unterhalb $\mathrm{T}_{\mathrm{CO}}$ beschränkt. In Volumenproben konnten bei Raumtemperatur, also in der paramagnetischen, isolierenden Phase, auch für Felder von bis zu 80 kOe und Spannungen bis zu $1 \mathrm{kV}$ keine Widerstandsänderung beobachtet werden [14,82. Dennoch haben Liu et al. Widerstandsänderungen von bis zu $1770 \%$ bei Raumtemperatur in dünnen PCMOSchichten gefunden, welche durch Spannungspulse unterschiedlicher Polarität und oberhalb einer kritischen Spannung verursacht werden (engl.: electric pulse induced resistance, EPIR) 25]. So kann z.B. der durch einen negativen Spannungspuls verursachte hochoh-

\footnotetext{
${ }^{(5)}$ Hieraus würde sich die überaus interessante Möglichkeit der Präparation von p-n-Übergängen auf Manganatbasis ergeben $[80]$.

${ }^{(6)}$ Das $\mathrm{CeO}_{2}$ besteht aus einem fcc-Gitter, dessen Plätze von $\mathrm{Ce}^{4+}$-Ionen besetzt sind. Die $\mathrm{O}^{2-}$-Ionen befinden sich in den Tetraeder-Lücken. Diese Kristallstruktur wird üblicherweise als $\mathrm{CaF}_{2}-\mathrm{Struktur}_{\text {be- }}$ zeichnet.
} 
mige Zustand durch einen positiven Spannungspuls zurück in den niederohmigen Zustand gebracht werden. Bemerkenswert ist die Tatsache, dass dieser Effekt auch in anderen Oxiden mit Perowskitstruktur wie z.B. in Cr-dotiertem $\mathrm{SrZrO}_{3}$ gefunden wurde 83, 84, 85 und somit einen allgemeineren Mechanismus vermuten lässt. Dabei ist anzumerken, dass der Effekt fast ausschließlich in einer isolierenden Phase des jeweiligen Systems auftritt oder einer isolierenden Grenzflächenschicht zugeordnet wird [86, 87]. Die jeweiligen Widerstandszustände sind über sehr lange Zeiträume (für Cr-dotiertes $\mathrm{SrTiO}_{3}$ bis zu 18 Monate ohne signifikante Änderung des Widerstandes [83|) stabil.

Die physikalische Ursache für das Auftreten eines polaritätsabhängigen Widerstandes ist eine momentan ungeklärte Fragestellung. So ist die Rolle der Grenzfläche Perowskit/Metallelektrode nicht abschließend geklärt, obwohl ein Einfluss unbestritten scheint 185,88,89, 90. Bedeutung kommt vermutlich auch der Valenz der beteiligten Übergangsmetalle zu, welche in den jeweiligen Widerstandszuständen unterschiedlich sein soll 90, 91. Ein Modell von Rozenberg et al. 92, 93, zeigt die Bedeutung von strukturellen und elektronischen Inhomogenitäten für das Widerstandsschalten in perowskitischen Materialien auf, wobei eine detaillierte Beschreibung der Inhomogenitäten nicht angegeben ist. Die Bedeutung von Inhomogenitäten wird in weiteren Arbeiten betont, auch in Verbindung mit den daraus resultierenden Modifikationen des Sauerstoffgehalts 89, 94, 88. Szot et al. [94 konnten in sauerstoffdefizitären $\mathrm{SrTiO}_{3}$ das Widerstandsverhalten einer einzelnen Versetzung beeinflussen, welches im sauerstoffgesättigten $\mathrm{SrTiO}_{3}$ nicht möglich ist. Auch Nian et al. führen das Widerstands-Schalten auf Sauerstoffdiffusion im Oxid nahe der Elektrode zurück [88. Ihre Analyse von zeit- und temperaturabhängigen Messungen an PCMO liefert deutliche Hinweise auf einen wesentlichen Einfluss der Sauerstoff-Diffusion.

Jooss et al. hingegen bringen das Widerstands-Schalten in PCMO wieder in den Zusammenhang mit dem Schmelzen der ladungsgeordneten Phase [22]. Ihre Beobachtung zeigen ein Auftreten der geordneten Phase (Ordnung von Zener-Polaronen) auch bei Raumtemperatur, welche durch einen genügend hohen Strom in eine ungeordnete Phase gebracht werden kann. In anderen Bereichen hingegen kann die Polaronen-geordnete Phase durch einen Strom induziert werden. 


\section{Kapitel 3}

\section{Magnetowiderstandseffekte}

Der Magnetowiderstand (engl.: magnetoresistance, MR) beschreibt die Änderung des elektrischen Widerstandes eines Systems bei Variation eines äußeren Magnetfeldes. Dabei können die physikalischen Mechanismen vielfältig sein. Grundsätzlich wird zwischen positiven und negativen Effekten unterschieden. Der positive Magnetowiderstand tritt immer auf und wird durch die Lorentzkraft auf bewegte Ladungsträger verursacht. Diese werden auf Kreisbahnen abgelenkt, wodurch sich die effektive freie Weglänge verringert, der Widerstand also ansteigt. Dieser Effekt lässt sich beispielsweise in Silber oder Gold gut beobachten. Oft wird der positive Magnetowiderstand aber von wesentlich größeren, negativen Effekten überlagert, wie z.B. dem anisotropen Magnetowiderstand (AMR) in ferromagnetischen Materialien. Dieser beruht auf der Spin-Bahn-Wechselwirkung und beschreibt die Abhängigkeit des elektrischen Widerstandes von der relativen Orientierung zwischen Stromrichtung und Magnetisierung.

Für großes Aufsehen haben die Entdeckungen weiterer negativer Magnetowiderstandseffekte wie des kolossalen Magnetowiderstandes (CMR), des Riesenmagnetowiderstandes (GMR) und des Tunnel-Magnetowiderstandes (TMR) geführt. Der CMR beschreibt dabei die Änderung des elektrischen Widerstandes in perowskitischen Manganaten, welche besonders nahe der Phasenübergangstemperatur ausgeprägt ist und mehrere Größenordnungen betragen kann (siehe Kapitel 2). Sowohl der GMR als auch der TMR werden dagegen nur in heterogenen Systemen beobachtet, welche aus Schichtpaketen oder granularen Materialien bestehen. Dabei werden ferromagnetische Elektroden entweder durch eine metallische, nichtmagnetische Zwischenschicht (GMR) oder durch eine dünne, isolierende Schicht (TMR) getrennt. Der elektrische Widerstand dieser Systeme ist von der relativen Orientierung der Magnetisierungen der ferromagnetischen Elektroden abhängig, wobei die Widerstandsänderungen auch bei Raumtemperatur sehr hoch sein können (180-220\% 4,5]). Daher haben solche Systeme industrielle Anwendungen zum Beispiel als Festplattenleseköpfe oder Magnetfeldsensoren gefunden.

Der TMR ist für diese Arbeit von zentraler Bedeutung und soll deshalb näher beschrieben werden. Er wurde bereits 1975 durch Jullière [2] experimentell entdeckt, wobei diese Ergebnisse zunächst nicht reproduziert werden konnten. Erst durch Fortschritte in der Probenpräparation, bedingt auch durch die Entdeckung des GMRs durch Grünberg et al. 955 und Baibich et al. [96 im Jahr 1988, konnten von Moodera et al. 1995 große 
TMR-Werte von ca. $10 \%$ auch bei Raumtemperatur gemessen werden [3]. In der aktuellen Forschung wird auch die Umkehrung des Effektes intensiv untersucht: Wenn die Änderung der relativen Orientierung der Magnetisierungen den elektrischen Strom beeinflusst, ist dann auch eine Änderung der relativen Orientierung durch den Strom möglich? Theoretische Berechnungen von Slonczewski und Berger [6,7] haben ergeben, dass diese Frage unter der Voraussetzung von hohen Stromdichten positiv beantwortet werden kann, was experimentell bestätigt wurde [8, 9]. Dieser Mechanismus wird auch als strominduziertes Magnetisierungsschalten (engl.: current-induced switching) bezeichnet.

Die für den TMR relevanten physikalischen Mechanismen sollen im Folgenden vorgestellt werden. Aufgrund der isolierenden Barriere kommt es beim TMR zum Auftreten des quantenmechanischen Tunneleffekts (Kapitel 3.1), dessen Verständnis unerlässig für die Diskussion weiterer Mechanismen ist. In Kapitel 3.2 werden diese Arbeiten auf den spinpolarisierten Fall erweitert. Anschließend soll in Kapitel 3.3 das strominduzierte Magnetisierungsschalten kurz skizziert werden und letztlich eine spezielle Klasse von MR-Systemen, die polykristallinen Manganate, vorgestellt werden (Kap. 3.4).

\subsection{Der Tunneleffekt}

Der Tunneleffekt beschreibt die Wahrscheinlichkeit $W(E)$, dass ein Teilchen ${ }^{(1)}$ eine Potentialschwelle durchdringen kann, deren potentielle Energie höher ist als die Energie des Teilchens. Dieser Effekt kann klassisch nicht beschrieben werden und verlangt daher eine quantenmechanische Behandlung. Bei der theoretischen Beschreibung können verschiedene, grundlegende Einschränkungen gemacht, bzw. fallen gelassen werden, s.d. die Berechnungen teilweise analytisch nicht mehr lösbar sind. Wichtige Unterscheidungskriterien sind: $i$ ) elastisches oder inelastisches Tunneln; $i i)$ ein-dimensionales oder dreidimensionales Tunneln; iii) rechteckförmige Potentialbarriere oder beliebig geformte Potentialbarriere und $i v$ ) zeitunabhängige oder zeitabhängige Behandlung. Für das Tunneln von Elektronen in Festkörpern ist weiterhin von Bedeutung, ob mit einem Modell freier Elektronen argumentiert wird oder Bandstruktureffekte berücksichtigt werden. Die nachfolgenden Argumentationen sind in detaillierter Ausführung auch in [97 zu finden.

\subsubsection{Elastisches Tunneln durch eine eindimensionale, rechteckige Potentialbarriere}

Die einfachste Situation wird durch einen elastischen Tunnelprozess eines auf eine eindimensionale, rechteckige Potentialbarriere zulaufenden Elektrons der Energie $E$ und Masse $m$ beschrieben. Die Potentialbarriere habe die Breite $d$ und die Höhe $\phi_{0}$, wie es in Abbildung 3.1 schematisch dargestellt ist. Durch das Anpassen der Wellenfunktionen der Bereiche I-III (Stetigkeit sowohl in $\psi$ als auch $\partial \psi / \partial z$ ) kann der Transmissionskoeffizient $T$ berechnet werden, welcher das Verhältnis von einfallendem zu emittiertem Elektronen-

(1) Im Folgenden seien nur Elektronen betrachtet. 


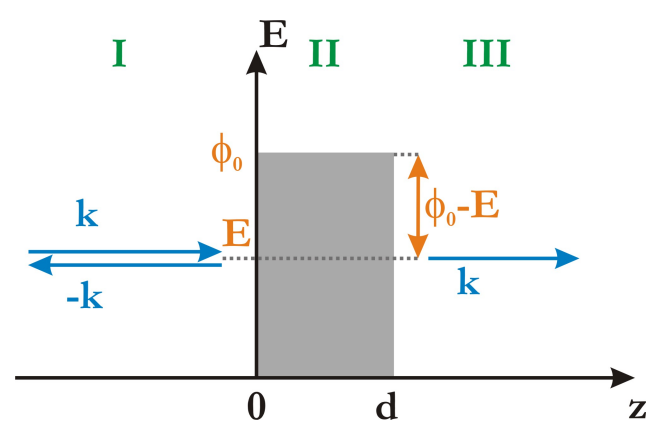

Abbildung 3.1. Schematische Darstellung des Tunneleffekts unter Verwendung einer eindimensionalen, rechteckigen Potentialbarriere der Höhe $\phi_{0}$ und Breite $d$.

strom angibt:

$$
T=\frac{1}{1+\frac{\left(k^{2}+\kappa^{2}\right)^{2}}{4 k^{2} \kappa^{2}} \sinh ^{2}(\kappa d)}
$$

Dabei ist $k$ der Wellenvektor der Welle, definiert über $k^{2}=2 m E / \hbar^{2}$ und $\kappa$ die charakteristische Abklinglänge, $\kappa^{2}=2 m\left(\phi_{0}-E\right) / \hbar^{2}$, welche von der effektiven Höhe $\left(\phi_{0}-E\right)$ der Potentialbarriere abhängt. Ist die Barriere sehr breit (und nicht gleichzeitig $E \approx \phi_{0}$ ), wird sinh $(\kappa d)$ sehr groß, s.d. $T$ gegen Null geht. Im umgekehrten Fall wird für dünne Barrieren der sinh $(\kappa d)$ klein, das Teilchen dringt fast sicher durch. Gleichung 3.1 kann unter der Annahme von $\kappa d \gg 1$ vereinfacht werden, es ergibt sich:

$$
T=\left(\frac{4 k \kappa}{k^{2}+\kappa^{2}}\right)^{2} \cdot \mathrm{e}^{-2 \kappa \mathrm{d}}
$$

Der Transmissionswahrscheinlichkeit nimmt folglich exponentiell mit zunehmender Barrierenbreite ab. Unter Verwendung eines realistischen Wertes für die Potentialbarrieren von wenigen eV beträgt $1 / \kappa$ weniger als $1 \AA$. Die Transmissionswahrscheinlichkeit ändert sich also bei Variation der Barrierenbreite um wenige $\AA$ drastisch.

Unter Verwendung eines zeitabhängigen Ansatzes ist es möglich, ebenfalls zu diesem Ergebnis zu gelangen. Auf eine explizite Rechnung wird an dieser Stelle verzichtet, dennoch soll in diesem Zusammenhang der wichtige Begriff des effektiven Tunnelmatrixelementes $M$ geklärt werden. Bei geeigneter Modifizierung des Potentials sind die Wellenfunktionen vollständig links $\left(\psi_{l}\right)$ und rechts $\left(\psi_{r}\right)$ der Barriere lokalisiert, es findet kein Tunnelprozess statt. Mit der zeitabhängigen Schrödingergleichung und einem geeignetem Ansatz für die Wellenfunktionen ergibt sich:

$$
M=\int \psi_{r}^{*} H_{T} \psi_{l} d z
$$

Der so genannte Transfer-Hamilton-Operator $H_{T}$ beschreibt dabei das Tunneln von einer Elektrode in die andere. In der Näherung stark abschwächender Potentiale kann Fermis Goldene Regel angewendet werden, welche die Übergangsrate eines gegebenen Zustandes auf der linken Seite in einen Satz von Zuständen gleicher Energie mit Zustandsdichte 
$\rho\left(E_{r}\right)$ auf der rechten Seite beschreibt. Hieraus kann der transmittierte Strom $J_{t}$ berechnet werden.

$$
J_{t}=2 e \frac{2 \pi}{\hbar}|M|^{2} \rho\left(E_{r}\right)
$$

Werden die expliziten Ausdrücke der Wellenfunktionen eingesetzt, ergibt sich der zu Gleichung 3.2 identische Transmissionskoeffizient.

Dennoch kann aus Gleichung 3.4 mehr Information gewonnen werden als aus dem reinen Transmissionskoeffizienten, da sich der Tunnelstrom scheinbar proportional zur elektronischen Zustandsdichte $\rho$ ergibt. Diese Tatsache motiviert einerseits weitere Modelle zum spinpolarisierten Tunneln wie das Jullière-Modell, andererseits muss zur Vorsicht gemahnt werden: Durch die explizite Berechnung des Tunnelmatrixelements ergibt sich oft keine einfache Proportionalität mehr, s.d. aus gemessenen Tunnelspektren nur in Spezialfällen direkt auf die Zustandsdichte geschlossen werden kann.

\subsubsection{Erweiterung auf den dreidimensionalen Fall}

Sowohl der zeitunabhängige als auch der zeitabhängige Ansatz lassen sich auf das dreidimensionale Problem erweitern. Die Potentialschwelle verlaufe unabhängig von $x$ und $y$ und die zum Potential parallele Komponente $k_{\|}$bleibe während des Tunnelprozesses erhalten. Hieraus ergibt sich letztlich, dass der Transmissionskoeffizient nur von der in $z$ Richtung zur Verfügung stehenden Energie $E_{z}=\hbar^{2} k_{z}^{2} / 2 m=E-\hbar^{2} k_{\|}^{2} / 2 m$ abhängt. Die Berechnung der mittleren Transmission durch die Mittelung über alle möglichen Werte von $\vec{k}$ einer gegebenen Fermi-Energie zeigt schließlich, dass die Transmission im wesentlichen dominiert wird von Elektronen, die nahezu senkrecht auf die Potentialschwelle zulaufen, deren $k_{\|}$-Wert also klein ist.

\subsubsection{Elastisches Tunneln in planaren Tunnelkontakten}

Im Folgenden soll ein planarer Tunnelkontakt mit einer eindimensionalen Potentialbarriere betrachtet werden. Dabei wird zunächst angenommen, dass beide Elektroden identisch sind und sich im thermischen Gleichgewicht befinden. Besondere Aufmerksamkeit wird der Spannungsabhängigkeit des Tunnelstroms gewidmet, wobei die Näherungen nach Simmons 98 angegeben werden.

Das Anlegen einer elektrischen Spannung $U$ an den Tunnelkontakt führt zu einer relativen Verschiebung eU der chemischen Potentiale der beiden Elektroden gegeneinander. Hierdurch fließst ein Nettotunnelstrom $J$ :

$$
J=\frac{e}{4 \pi^{2} \hbar d^{2}}\left\{\phi_{0} \cdot \mathrm{e}^{-\mathrm{A} \cdot \mathrm{d} \cdot \sqrt{\phi_{0}}}-\left(\phi_{0}-\mathrm{eU}\right) \cdot \mathrm{e}^{-\mathrm{A} \cdot \mathrm{d} \cdot \sqrt{\phi_{0}-\mathrm{eU}}}\right\}
$$

Der Parameter $A$ ist gegeben durch $A=\sqrt{8 m} / \hbar$. Simmons $[98$ hat diesen Tunnelstrom für verschiedene Grenzfälle genähert, wobei hier insbesondere kleine und mittlere Spannungen interessieren.

(i) Bereich der kleinen Spannungen, $e U \ll \phi_{0}$ 


$$
J=\frac{e^{2}}{8 \pi^{2} \hbar} \frac{A \cdot \phi_{0}}{d} \cdot U \cdot \mathrm{e}^{-\mathrm{A} \cdot \mathrm{d} \cdot \sqrt{\phi_{0}}}
$$

Es ergibt sich wie für den eindimensionalen Fall der charakteristische exponentielle Abfall, abhängig von der Barrierenbreite $d$ und $\sqrt{\phi_{0}}$. Weiterhin zeigt sich, dass der Tunnelstrom proportional zur Spannung $U$ ist. Dies ist gleichbedeutend mit einem Ohmschen Widerstandsverhalten.

(ii) Bereich der mittleren Spannungen, $e U<\phi_{0}$

Für den Bereich mittlerer Spannung lässt sich Gleichung 3.5 nicht vereinfachen. Um aber Informationen über das Verhalten in Abhängigkeit der angelegten Spannung zu erhalten, kann die Leitfähigkeit $G(U)=d J / d U$ berechnet werden:

$$
\begin{gathered}
G(U)=G(0)+G(0) \cdot\left(\frac{e^{2} m}{4 \hbar^{2}} \frac{d^{2}}{\phi_{0}}\right) \cdot U^{2} \\
G(0)=\frac{e^{2}}{4 \pi^{2} \hbar^{2} d} \sqrt{2 m \phi_{0}} \cdot \mathrm{e}^{-\mathrm{A} \cdot \mathrm{d} \cdot \sqrt{\phi_{0}}}
\end{gathered}
$$

Die Leitfähigkeit ändert sich als Funktion der angelegten Spannung, es liegt kein Ohmsches Widerstandsverhalten vor. Einerseits kann deshalb durch das Auftreten von nichtlinearen Kennlinien ein Tunnelprozess vermutet werden ${ }^{(2)}$ andererseits können mit Hilfe von Gleichung 3.7 Informationen über die Barriere (Breite $d$ und Höhe $\phi_{0}$ ) gewonnen werden.

Die Kennlinie kann neben der Barrierenhöhe und -breite aber auch Informationen über deren Asymmetrie enthalten. Dazu seien Elektroden unterschiedlicher Austrittsarbeiten $\Phi_{1}$ und $\Phi_{2}$ betrachtet. Es ergibt sich ein intrinsisches Kontaktpotential $\left(\Phi_{2}-\Phi_{1}\right) / e$, wodurch die Potentialschwelle asymmetrisch wird. Die Spannungsabhängigkeit des Tunnelstromes ist somit zusätzlich abhängig von der Polarität der Spannung. Eine Berechnung der Leitfähigkeit für diesen Fall, die so genannte Brinkman-Formel, ist in [100] gegeben.

\subsubsection{Temperatur- und Bandstruktureffekte}

Aufgrund der unterschiedlichen Energieskalen der Höhe der Potentialbarriere einerseits, welche in der Größenordnung von $1 \mathrm{eV}$ liegt, und der thermischen Energie andererseits, welche bei Raumtemperatur $25 \mathrm{meV}$ beträgt, zeigt der Tunnelstrom nur eine sehr geringe Temperaturabhängigkeit. Im Bereich tiefer Temperaturen kann er als temperaturunabhängig betrachtet werden.

Im Gegensatz zur Temperatur haben Bandstruktureffekte einen immensen Einfluss auf den Transmissionskoeffizienten. Bislang wurde von einem Modell freier Elektronen ausgegangen. Wird diese Annahme fallengelassen, so muss insbesondere die effektive Masse $m^{*}$ der Elektronen berücksichtigt werden, welche sich von der Masse $m$ der freien Elektronen unterscheidet. Die für den Transmissionskoeffizienten maßgebliche Abklinglänge $\kappa$ lässt

\footnotetext{
${ }^{(2)}$ Das alleinige Auftreten einer nichtlinearen Kennlinie ist hier aber nur eine notwendige und keinesfalls eine hinreichende Bedingung $[99]$.
} 
sich folgendermaßen darstellen:

$$
\kappa^{2}=k_{\|}^{2}+\frac{2 m^{*}}{\hbar^{2}}\left(\phi_{0}-E\right)
$$

Elektronen großer effektiver Massen tunneln also weniger effektiv. Dieses ist besonders für die $3 d$-Übergangsmetalle von Bedeutung, in denen sowohl die $3 d$ - als auch die $4 s$ Elektronen zum Tunnelstrom beitragen können. Da aber die $4 s$-Elektronen im Allgemeinen eine geringere effektive Masse aufweisen, wird der Tunnelstrom vorwiegend von ihnen getragen.

\subsection{Tunnel-Magnetowiderstand}

\subsubsection{Jullière-Modell}

Der Tunnel-Magnetowiderstands-Effekt bezeichnet die Abhängigkeit des elektrischen Widerstandes von der relativen Orientierung der Magnetisierungen zweier ferromagnetischer Elektroden, welche durch eine dünne isolierende Schicht getrennt sind. Bereits im Jahre 1975 hat Jullière [2] ein einfaches Modell basierend auf der spinaufgespaltenen Elektronenstruktur ferromagnetischer Festkörper und Gleichung 3.4 gegeben. Zwei Einschränkungen sind wesentlich für das Modell:

(i) Spinerhaltung

Spin-flip-Prozesse während des Tunnelns werden vernachlässigt. Dieses ist für defektfreie Barrieren, tiefe Temperaturen und kleine Spannung eine gute Näherung, die in allen weiteren Modellen ebenfalls verwendet wird.

(ii) Proportionalität der Tunnelwahrscheinlichkeit jeder Spinrichtung zur jeweiligen Zustandsdichte an $E_{F}$

Jede Spinrichtung wird für sich betrachtet. Dieses entspricht dem gängigen ZweiStrom-Modell 101. Die Proportionalität geht dabei auf Gleichung 3.4 zurück, wobei Bandstruktur-Effekte, wie beispielsweise die Berücksichtigung effektiver Massen, ausgelassen werden.

Abbildung 3.2 zeigt die schematischen Zustandsdichten der ferromagnetischen Elektroden, welche durch eine Barriere getrennt sind. Auf der linken Seite ist die parallele Konfiguration gezeigt: Die chemischen Potentiale sind durch das Anlegen einer Spannung leicht gegeneinander verschoben, es fließst ein Nettotunnelstrom von der linken (L) zur rechten (R) Elektrode. Aufgrund der spinaufgespaltenen Zustandsdichte und der Lage der Fermienergie ist die Zahl der Zustände an der Fermienergie für die beiden Spinrichtungen unterschiedlich (Zustandsdichte an $E_{F}$ für Spin $\uparrow(\downarrow): n_{\uparrow(\downarrow)}\left(E_{F}\right)$ ). Für den diskutierten Fall der parallelen Konfiguration ergibt sich für beide Elektroden eine hohe (niedrige) Zustandsdichte an $E_{F}$ für die Spin-ab (auf) Richtung. Aufgrund der Proportionalität der Tunnelwahrscheinlichkeit zur Zustandsdichte an $E_{F}$ ist diese für die Spin-ab Elektronen 


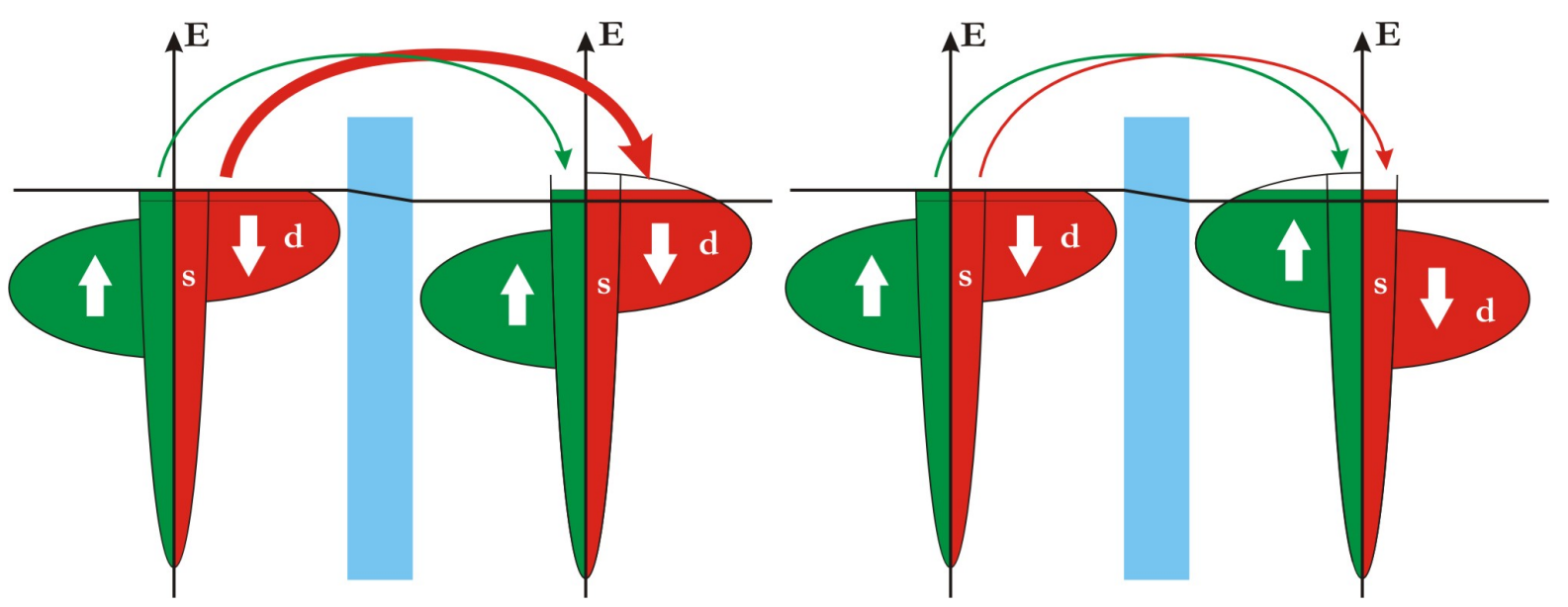

Abbildung 3.2. Schematische Darstellung des Jullière-Modells. Links: parallele Konfiguration. Rechts: antiparallele Konfiguration. Nach 102

sehr hoch (viele Elektronen stehen in der linken Elektrode an der Fermikante zur Verfügung, während in der rechten Elektrode viele freie Zustände verfügbar sind), wohingegen sie für die Spin-auf Elektronen sehr klein ist (wenige Elektronen stehen zur Verfügung, nur wenige freie Zustände sind verfügbar). Es fließt ein hoher Tunnelstrom, der fast ausschließlich von den Spin-ab Elektronen getragen wird. Formal ausgedrückt ergibt sich für die Leitfähigkeit:

$$
G_{P} \propto\left[n_{\uparrow}^{L}\left(E_{F}\right) \cdot n_{\uparrow}^{R}\left(E_{F}\right)+n_{\downarrow}^{L}\left(E_{F}\right) \cdot n_{\downarrow}^{R}\left(E_{F}\right)\right]
$$

Für die antiparallele Konfiguration (Abb. 3.2, rechts) dreht sich die Zustandsdichte bezüglich der Spinrichtung in einer Elektrode gerade um. Die Leitfähigkeit ist also gegeben durch:

$$
G_{A P} \propto\left[n_{\uparrow}^{L}\left(E_{F}\right) \cdot n_{\downarrow}^{R}\left(E_{F}\right)+n_{\uparrow}^{L}\left(E_{F}\right) \cdot n_{\downarrow}^{R}\left(E_{F}\right)\right]
$$

Zwar stehen viele Spin-ab Elektronen zur Verfügung, diese finden aber nur wenige freie Zustände in der rechten Elektrode. Andererseits sind nur wenige Spin-auf Elektronen verfügbar, welche in die rechte Elektrode tunneln können. Der Gesamttunnelstrom ist folglich deutlich kleiner als für die parallele Konfiguration.

Offensichtlich bestimmt das Verhältnis der Zustandsdichten der jeweiligen Spinrichtung an der Fermikante die Differenz der Leitfähigkeiten für den parallelen und antiparallelen Zustand wesentlich. Deshalb wird der wichtige Begriff der Spinpolarisation $P$ eingeführt:

$$
P=\frac{n_{\downarrow}\left(E_{F}\right)-n_{\uparrow}\left(E_{F}\right)}{n_{\downarrow}\left(E_{F}\right)+n_{\uparrow}\left(E_{F}\right)}
$$

Der TMR beschreibt die Differenz der Leitfähigkeiten in paralleler und antiparalleler Konfiguration normiert auf die Leitfähigkeit in antiparalleler Konfiguration. Unter Verwendung von Gleichungen 3.10 - 3.12 ergibt sich:

$$
\mathrm{TMR}=\frac{\mathrm{G}_{\mathrm{P}}-\mathrm{G}_{\mathrm{AP}}}{\mathrm{G}_{\mathrm{AP}}}=\frac{\mathrm{R}_{\mathrm{AP}}-\mathrm{R}_{\mathrm{P}}}{\mathrm{R}_{\mathrm{P}}}=\frac{2 \mathrm{P}_{\mathrm{L}} \mathrm{P}_{\mathrm{R}}}{1-\mathrm{P}_{\mathrm{L}} \mathrm{P}_{\mathrm{R}}}
$$


Der TMR, der üblicherweise in Prozent angegeben wird, ist direkt mit der Spinpolarisation verbunden und wird größer, je höher die Spinpolarisation der jeweiligen Elektroden ist. Dies motiviert die Verwendung hoch-spinpolarisierter Elektroden wie der bereits vorgestellten Manganate (s. Kap. 2), welche eine 100\%ige Spinpolarisation aufweisen [10. Weitere Materialien, denen diese Eigenschaft zugesprochen wird und die aktuell intensiv untersucht werden, sind z.B. die so genannten Heusler-Legierungen 103, 104, Magnetit $\left(\mathrm{Fe}_{3} \mathrm{O}_{4}\right)$ 105, 106 und $\mathrm{CrO}_{2}$ [106, 107, 108.

\subsubsection{Erweiterungen des Jullière-Modells}

\section{Modell von Slonczewski und Bratkovsky}

Das Jullière-Modell liefert besonders bei sehr dünnen und/oder sehr niedrigen Barrieren keine zufriedenstellenden Ergebnisse, da der auftretende Überlapp der Wellenfunktionen im Modell nicht berücksichtigt wird. Aus diesem Grunde berechnete Slonczewski 1989 die Leitfähigkeit über die Anpassung der Wellenfunktionen wie in Abschnitt 3.1.1, wobei er das Modell aber auf den spinpolarisierten Fall erweiterte [109]. Da bei diesen Berechnungen die Kenntnis der Wellenfunktionen vorausgesetzt wird, nahm er ein Modell freier Elektronen an. Das wesentliche Ergebnis dieser Berechnungen ist, dass die effektive Polarisation $P_{\text {eff }}$ mit der Barrierenhöhe über die Abklinglänge $\kappa=\sqrt{2 m\left(\phi_{0}-E_{F}\right) / \hbar}$ verknüpft ist:

$$
P_{e f f}=\frac{k_{\uparrow}-k_{\downarrow}}{k_{\uparrow}+k_{\downarrow}} \cdot \frac{\kappa^{2}-k_{\uparrow} k_{\downarrow}}{\kappa^{2}+k_{\uparrow} k_{\downarrow}}
$$

Dabei bezeichnen $k_{\uparrow}$ und $k_{\downarrow}$ die Fermi-Wellenvektoren für das Spin-auf bzw. ab Band. Im Fall freier Elektronen gilt $k_{\uparrow(\downarrow)} \propto n_{\uparrow(\downarrow)}\left(E_{F}\right)$, der erste Term spiegelt daher die übliche Definition der Spinpolarisation wider. Der neu hinzugekommene Term, der Werte zwischen -1 und 1 annehmen kann, ergibt sich aus der Tatsache, dass die Eindringtiefe der Wellenfunktion in der Barriere von der Spinrichtung abhängt. Für genügend kleine Barrierenhöhen $(\kappa \rightarrow 0)$ kann dieser Grenzflächenfaktor das Vorzeichen der Polarisation drehen, wohingegen für hohe Barrieren $(\kappa \rightarrow \infty)$ das Ergebnis von Jullière reproduziert wird.

Eine Modifikation dieser effektiven Spinpolarisation hat Bratkovsky berechnet [110], indem er den Einfluss einer effektiven Masse $m^{*}$ innerhalb der Barriere berücksichtigte:

$$
P_{e f f}=\frac{k_{\uparrow}-k_{\downarrow}}{k_{\uparrow}+k_{\downarrow}} \cdot \frac{\kappa^{2}-\left(m^{*}\right)^{2} \cdot k_{\uparrow} k_{\downarrow}}{\kappa^{2}+\left(m^{*}\right)^{2} \cdot k_{\uparrow} k_{\downarrow}}
$$

Wird die effektive Masse in der Barriere kleiner, so kann z.B. ein nach Gleichung 3.14 berechnetes $P_{\text {eff }}$ negativ sein, wohingegen es durch die Berücksichtigung der veränderten effektiven Masse innerhalb der Barriere zu einem positiven Wert kommt.

\section{First-principle Rechnungen vs. Experiment: Fe/MgO/Fe}

Alle bisher vorgestellten Konzepte basieren auf einem Modell freier Elektronen für die ferromagnetischen Elektroden. Eine Betrachtung der realen Bandstrukturen, besonders 
der ferromagnetischen $3 d$-Übergangsmetalle Fe, Ni und Co, zeigt, dass dieses Modell eigentlich keine Anwendung finden kann. Da die Tunnelwahrscheinlichkeit stark von der effektiven Masse der Elektronen abhängt, muss die Bandstruktur in TMR-Berechungen berücksichtigt werden.

Butler et al. haben detaillierte Analysen für das epitaktische System $\mathrm{Fe} / \mathrm{MgO} / \mathrm{Fe}$ durchgeführt und hohe TMR-Werte vorausgesagt [111. Vereinfacht dargestellt basieren ihre Ergebnisse auf der Forderung, dass die Symmetrie der Wellenfunktionen während des Tunnelprozesses erhalten bleibt. Dabei zeigen verschiedene Zustände an der Fermikante aufgrund der komplexen Bandstruktur des MgOs stark unterschiedliche Abklingraten, wobei ein s-artiger Zustand, welcher nur für die Majoritätselektronen auftritt, die geringste Abklinglänge aufweist. Dieser Zustand dominiert die Leitfähigkeit demnach in der parallelen Konfiguration. In der antiparallelen Konfiguration hingegen muss der s-artige Zustand jenseits der Barriere abklingen, da er in der Minoritäts-Zustandsdichte nicht auftritt. Das Elektron wird somit vollständig reflektiert. Hieraus resultiert ein großer TMR. Weiterhin zeigen die Rechnungen, dass der TMR mit zunehmender Barrierendicke ansteigt. Dieses ist auf die zunehmende Unterdrückung des Minoritätskanals zurückzuführen, der sehr viel schneller in der Barriere abfällt als der Majoritätszustand.

Die Vorhersagen von Butler et al. wurden nach Überwindung vieler experimenteller Schwierigkeiten bestätigt: Sowohl Yuasa et al. [4] als auch Parkin et al. [5] haben von sehr hohen TMR-Werten bei Raumtemperatur berichtet und weitere Berechnungen wie die Erhöhung des TMRs durch Verbreiterung der Barrierendicke verifiziert. Die theoretischen Arbeiten und die entsprechenden Experimente zeigen die Bedeutung der Bandstruktur für den TMR sowie das enorme Potential der verwendeten MgO-Barrieren.

Die Bedeutung von $\mathrm{MgO}$ als Barrierenmaterial zeigt sich auch in Experimenten, in denen das amorphe CoFeB als Elektrode verwendet wird. Es konnten Effekte von $230 \%$ bei Raumtemperatur erreicht werden 112,113. Unter Verwendung der hoch-spinpolarisierten Materialien lassen sich noch höhere TMR-Werte erzielen, allerdings sind diese großen Effekte auf tiefe Temperaturen beschränkt, siehe z.B. |114.

\subsubsection{Temperatur- und Spannungsabhängigkeit}

Sowohl die Temperatur- als auch die Spannungsabhängigkeit des TMRs sind noch immer Gegenstand kontroverser Diskussionen in der aktuellen Forschung. Einen unbestritten wichtigen Einfluss auf die Temperaturabhängigkeit hat die Spinpolarisation, welche ebenfalls temperaturabhängig ist. Aufgrund der Bedeutung der Grenzflächen Ferromagnet/Isolator muss aber die Temperaturentwicklung der Spinpolaristation der Grenzfläche betrachtet werden, welche sich von der des Volumens unterscheidet. So fanden Park et al. $\mid 115$ eine sehr gute Übereinstimmung in der Temperaturabhängigkeit der polarisierten Ladungsträgerdichte verglichen mit dem Kleinfeld-Magnetowiderstand in polykristallinem LSMO (s. Kap. 3.4). Auch Modeera et al. berichten von einer Übereinstimmung in Grenzflächenmagnetisierung (und damit Polarisation) und Magnetowiderstand [116].

In den bisherigen Betrachtungen wurde der Tunnelprozess immer als elastisch angenommen, d.h. sowohl die Energie als auch der Spin der tunnelnden Elektronen bleibt 
erhalten. Zur Beschreibung der Experimente ist diese Annahme nicht ausreichend, da wichtige Eigenschaften wie der starke Abfall des TMR-Wertes mit zunehmender Temperatur oder Spannung nicht beschrieben werden können [117]. Durch die Wechselwirkung mit elementaren Anregungen (Phononen, Magnonen) kommt es zu inelastischen Prozessen, welche Einfluss auf den TMR haben. Dabei wird üblicherweise unterschieden zwischen Prozessen in den Elektroden und in der Barriere. Magnonen-unterstütztes Tunneln 118 führt zur Erniedrigung des TMR-Wertes, wohingegen Phononen-unterstütztes Tunneln diesen Effekt aufgrund seiner spinerhaltenden Eigenschaft abschwächen kann [119,120. Die relativen Wertigkeiten dieser beiden Beiträge hängen stark von den mikrostrukturellen Eigenschaften der Ferromagnet/Isolator-Grenzfläche ab, s.d. sogar eine leichte Erhöhung des TMR mit zunehmender Temperatur beobachtet werden konnte [120].

\section{Das Glazman-Matveev-Modell}

Neben den elementaren Anregungsmoden und der Temperaturabhängigkeit der Spinpolarisation können Defektzustände in der Barriere enormen Einfluss auf den spinpolarisierten Tunnelprozess ausüben. Solche Defektzustände werden in oxidischen Materialien besonders durch Sauerstoff-Fehlstellen verursacht und gewinnen mit zunehmender Barrierendicke mehr Einfluss auf die Tunnelleitfähigkeit. Abbildung 3.3 zeigt schematisch mögliche Tunnelkanäle: Der Tunnelprozess über die beiden Kanäle, welche das direkte $(n=0)$ Tunneln, bzw. das resonante Tunneln über einen Defektezustand $(n=1)$ darstellen, erfolgt elastisch. Für $n \geq 2$ erfolgt das Tunneln inelastisch, da die Zwischenzustände in der Regel nicht die gleiche Energie aufweisen. Glazman und Matveev haben die Leitfähigkeit eines solchen Transports über Zwischenzustände berechnet [121], wobei die Gesamtleitfähigkeit $G$ durch die Summe der Leitfähigkeiten aller möglichen Kanäle gegeben ist. Für tiefe Temperaturen können diese einzelnen Leitfähigkeiten über die Zwischenzustände (also $n \geq 1$ ) genähert werden durch:

$$
G_{n}=a_{n} \cdot U^{n-\frac{2}{n+1}}
$$

Die Konstanten $a_{n}$ beinhalten die typischen exponentiellen Abfälle, wobei aber aufgrund der Zwischenzustände eine Art effektive Barrierenbreite von $d /(n+1)$ in die Abklinglänge eingeht, da der Tunnelprozess in $n+1$ Prozesse unterteilt werden kann. Diese Kanäle fallen demnach deutlich langsamer in der Barriere ab, weshalb sie für dicke Barrieren dominant werden können.

Da die Leitfähigkeit des direkten Kanals $(n=0)$ unabhängig von der angelegten Spannung ist, kann der Zusammenhang zwischen Strom und Spannung angegeben werden:

$$
\begin{gathered}
I(U)=G(U) \cdot U \\
G(U)=G_{0}+G_{1}+G_{2} \cdot U^{4 / 3}+G_{3} \cdot U^{5 / 2}+\ldots
\end{gathered}
$$

Die inelastischen Kanäle tragen also mit zunehmender Spannung mehr zum Tunnelstrom bei, da immer mehr Zwischenzustände energetisch zugänglich werden. Die Berechnung der Temperaturabhängigkeit führt zu einem ähnlichen Ergebnis. Die zunehmende Bedeutung der inelastischen Kanäle hat Auswirkungen auf den TMR: Wenn die lokalisierten Zwi- 


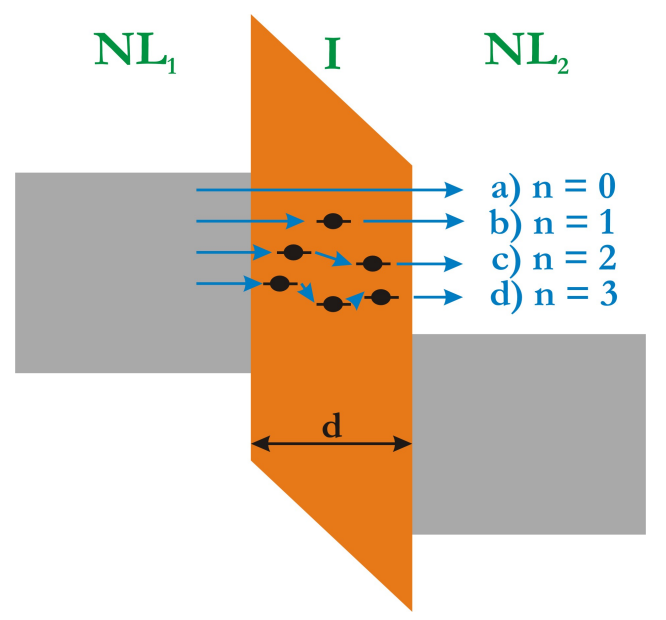

Abbildung 3.3. Schematische Darstellung der möglichen elastischen und inelastischen Tunnelkanäle beim Tunneln durch eine Barriere großer Defektdichte. NL: Normalleiter; I: Isolator. Nach 44 .

schenzustände auch den Spinzustand des Elektrons ändern, so sinkt demnach der TMR mit zunehmender Spannung, bzw. zunehmender Temperatur.

\subsection{Strominduziertes Magnetisierungsschalten}

Beim Tunnel-Magnetowiderstand wird der Strom über die relative Orientierung der Magnetisierungen der beiden Elektroden beeinflusst. Dieser Umstand fordert nun auf ganz natürliche Weise die Frage nach der Umkehrung dieses Effektes heraus: Kann die relative Orientierung der Magnetisierungen durch den Strom beeinflusst werden? Nach Slonczewski und Berger übt ein spinpolarisierter Strom, der in einen Ferromagneten geleitet wird, ein Drehmoment auf die Magnetisierung aus, welches bei genügend hohen Stromdichten $\left(10^{6}-10^{8} \mathrm{~A} / \mathrm{cm}^{2}\right)$ in der Lage ist, diese zu beeinflussen $[109,7]$. Dieser Effekt kann ausgenutzt werden, um die relative Orientierung der Magnetisierungen zweier Ferromagneten zu steuern. Hierzu müssen zwei wichtige Einschränkungen gemacht werden. Zum einen sind die beiden Ferromagneten durch eine nichtmagnetische Zwischenschicht getrennt, deren Dicke kleiner ist als ihre Spin-Diffusionslänge. Zum anderen muss eine Asymmetrie vorliegen, d.h. ein Ferromagnet kann leichter ummagnetisiert werden als der andere (unterschiedliche Koerzitivfelder oder Schichtdicken). Sie werden im Folgenden als frei, bzw. fest bezeichnet. Fließen nun Elektronen ${ }^{(3)}$ bei genügend hoher Stromdichte über die feste zur freien Elektrode, so ist eine parallele Konfiguration stabil. Wird die Polarität geändert, so ist eine antiparallele Konfiguration stabil. Dieser Effekt, der auch experimentell bestätigt wurde (s. z.B. 122]), soll im Folgenden plausibel gemacht werden.

Zunächst sei ein spinpolarisierter Strom betrachtet, der von einem nichtmagnetischen Material kommend auf einen Ferromagneten trifft (Abbildung 3.4). Der einfallende Strom

\footnotetext{
${ }^{(3)}$ Für die folgenden Argumentationen ist mit der Stromrichtung die Richtung der Elektronen gemeint, im Gegensatz zur allg. üblichen technischen Stromrichtung.
} 


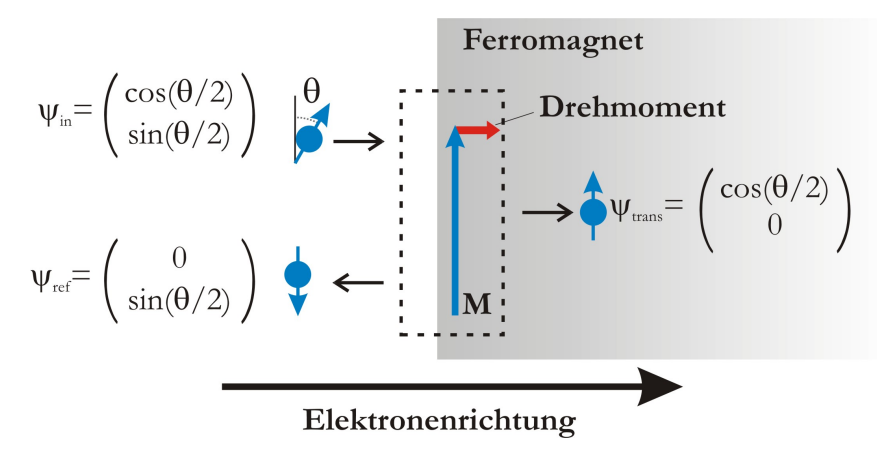

Abbildung 3.4. Schematische Darstellung des Spin-Filter-Effekts, dem ein Drehmoment auf die Magnetisierung zugrunde liegt. Nach [123].

sei entlang einer Achse, die um den Winkel $\theta$ gegen die Magnetisierung des Ferromagneten gedreht ist, polarisiert. Die normalisierte Wellenfunktion $\psi_{\text {in }}$ eines einfallenden Elektrons kann also durch eine Superposition von Spin-auf und Spin-ab Komponenten in Bezug auf die Quantisierungsachse, welche durch $\vec{M}$ definiert ist, geschrieben werden. Die Amplituden sind demnach $\cos (\theta / 2)$, bzw. $\sin (\theta / 2)$, woraus sich eine Transversalkomponente von $\sin (\theta)$ ergibt. Aufgrund der spinaufgespaltenen Zustandsdichte im Ferromagneten kommt es an der Grenzfläche zu einer spinabhängigen Transmission und Reflektion, wodurch die Wellenfunktionen der transmittierten, bzw. reflektierten Elektronen unterschiedliche Superpositionen der Spin-auf und Spin-ab Komponenten sind, was unweigerlich zu einer Änderung der transversalen Komponente führt. Die Differenz der transversalen Komponenten wird von der Grenzfläche absorbiert und wirkt demzufolge als Drehmoment auf die Magnetisierung. Der beschriebene Effekt wird in der Literatur auch als spin-filtering bezeichnet 109.

In realistischen Fällen erfolgt die Absorption der transversalen Komponente durch den Spin-Filter-Effekt aber nicht immer so vollständig wie es in Abbildung 3.4 dargestellt ist. Sowohl der reflektierte als auch der transmittierte Strom können weiterhin Transversalkomponenten tragen [124. Um aber die aktuelle Polarisation der reflektierten und transmittierten Elektronen zu ermitteln, muss über die gesamte Fermifläche summiert werden. Diese Summation führt zu zwei weiteren Effekten, die letztlich in den meisten Fällen eine vollständige Eleminierung der transversalen Spinkomponente verursachen. Zum einen können die Amplituden der reflektierten und transmittierten Elektronen komplex sein, s.d. es zu einer Rotation der Spins kommt. Durch Summation über die Fermifläche mittelt sich diese Rotation aber heraus, es kommt zu einer Reduzierung der transversalen Komponente. Als zweiter Effekt entsteht eine räumliche Präzession der Spins im Ferromagnet aufgrund der spinaufgespaltenen Zustandsdichte. Die Frequenz dieser Präzession ist aber unterschiedlich für Elektronen aus verschiedenen Bereichen der Fermifläche. Daher führt auch dieser Effekt nach Summation über die gesamte Fermifläche zu einer Reduzierung der transversalen Komponente [124]. Nach Stiles et al. kann daher die transversale Komponente eines einfallenden Spinstroms in guter Näherung als proportional zum auf die Magnetisierung wirkenden Drehmoment angesehen werden [124. Dabei wird die transversale Komponente schon an der Grenzfläche absorbiert. 

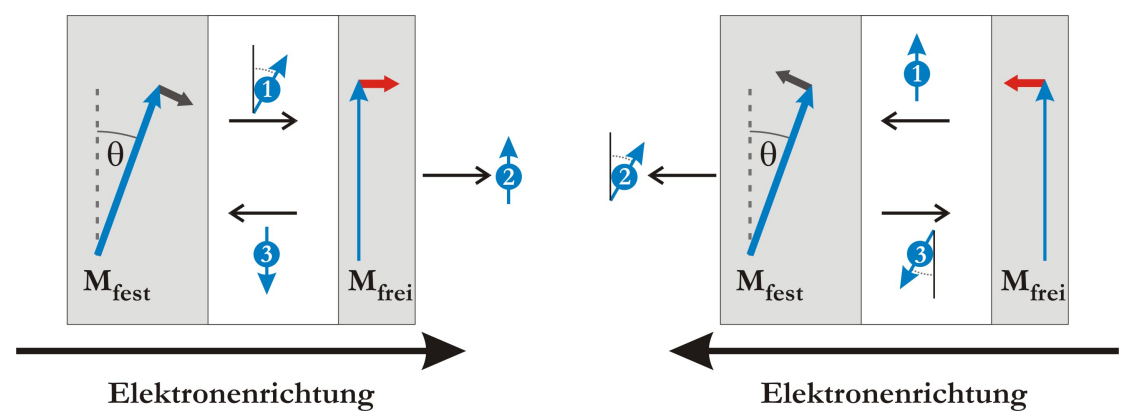

Abbildung 3.5. Physikalische Plausibilisierung des strominduzierten Magnetisierungsschalten. Links: Parallele Konfiguration stabil; rechts: antiparallele Konfiguration stabil. Nach 123 .

Nun sollen die aus den oben beschriebenen Effekten resultierenden Konsequenzen für das bereits vorgestellte Schichtpaket besprochen werden (Abbildung 3.5). Bisher wurde immer von einem bereits spinpolarisierten, einfallenden Strom ausgegangen, welcher im Schichtpaket durch das Einbringen der zweiten ferromagnetischen Elektrode erzeugt wird, dessen Magnetisierung leicht um einen Winkel $\theta$ gegen die Magnetisierung des zweiten Ferromagneten gekippt ist. Die aus dem Ferromagneten austretenden Elektronen sind spinpolarisiert und werden an der Grenzfläche zum Nichtmagneten nicht beeinflusst, da dessen Zustandsdichte nicht spinabhängig ist. Auf der linken Seite von Abbildung 3.5 fließen die Elektronen von der festen zur freien Schicht. Sie werden also zunächst von der festen Schicht polarisiert (1) und treffen dann auf die Grenzfläche zur freien Elektrode. Aufgrund der oben beschriebenen Effekte wird die transversale Komponente absorbiert und es wirkt ein Drehmoment. Ein Teil der Elektronen wird dann transmittert (2), der andere Teil reflektiert (3). Der reflektierte Strom trifft also wieder auf die Grenzfläche zum festen Ferromagneten, wiederum wird die transversale Komponente absorbiert und es wirkt ein Drehmoment. Dieses hat aber aufgrund der vorausgesetzten Asymmetrie keine Wirkung auf die feste Magnetisierung. Die freie Magnetisierung hingegen wird durch das wirkende Drehmoment so beeinflusst, dass sich eine parallele Konfiguration einstellt. Wird die Polarität des Stromes geändert, ergibt sich eine ähnliche Situation, allerdings ändern sich auch die Vorzeichen der wirkenden Drehmomente. Weiterhin muss die Asymmetrie berücksichtigt werden: Zunächst wird durch die freie Elektrode ein spinpolarisierter Strom erzeugt, der auf die feste Elektrode trifft. Das resultierende Drehmoment hat aber keinen Einfluss auf die feste Magnetisierung. Der reflektierte Strom trifft auf die freie Elektrode, das wirkende Drehmoment führt zu einer stabilen antiparallelen Konfiguration. Die Notwendigkeit der Berücksichtigung des reflektierten Stroms, der die Zwischenschicht zweimal passiert, wurde durch Albert et al. experimentell bestätigt [125].

Bei unterschiedlicher Polarität des Stromes sind somit verschiedene Konfigurationen stabil. Dabei wird die freie Magnetisierung relativ zur festen gedreht, unabhängig von der speziellen Orientierung der festen Magnetisierung. Ein äußeres magnetisches Feld favorisiert eine parallele Konfiguration der beiden Magnetisierungen, s.d. die kritischen Ströme feldabhängig sind. Da die parallele Orientierung im Feld leichter zu erreichen 
ist, sinkt der kritische Strom für die parallele Orientierung, für die antiparallele steigt er an $[122$. Die Feldabhängigkeit der kritischen Stromdichte ist ein allgemeines Merkmal des strominduzierten Magnetisierungsschaltens.

Die kritische Stromdichte ist weiterhin abhängig von der Spinpolarisation der Elektroden [126]. Unter Verwendung hoch-spinpolarisierter Materialien wie der Manganate sollten sich also geringe kritische Stromdichten erreichen lassen.

\subsection{Korngrenzen-Magnetowiderstand in Manganaten}

Aufgrund ihrer hohen Spinpolarisation sind Manganate für die Anwendungen in TMRSystemen besonders interessant. Neben den konventionellen Dünnschicht-Systemen sind dabei aber auch andere Anordnungen wie granulare Systeme denkbar. Für großes Aufsehen sorgte in diesem Zusammenhang die Arbeit von Hwang et al. [27], welche einen enormen Unterschied in der Magnetfeldabhängigkeit des Widerstandes zwischen einkristallinen und polykristallinen LSMO-Proben aufzeigte. Neben dem typischen CMR-Verhalten, welches für beide Probenarten auftritt, wird in den Polykristallen eine rapide Abnahme des Widerstandes (bis zu 20\%) in kleinen Feldern gefunden. Der zusätzliche KleinfeldMagnetowiderstand wird von den Autoren durch das spinpolarisierte Tunneln zwischen ferromagnetischen Körnern, welche durch eine isolierende Barriere getrennt sind, erklärt. Dieser mögliche Transportmechanismus ist in der Literatur nicht unstrittig, wobei die Diskussion besonders den Charakter der Barriere betrifft. Das Auftreten isolierender Phasen an Korn- bzw. Grenzflächen wird zwar durch Bibes et al. mittels NMR-Analysen bestätigt [127, 128], dennoch sind weitere Transportprozesse denkbar. Um den Einfluss der Korngrenzen und deren Eigenschaften näher zu untersuchen, wurden u.a. definierte Korngrenzenkontakte auf STO- und LAO-Bikristall-Substraten präpariert, siehe z.B. 129, 130, 131, 132, 133, 134, 135, 136 und besonders 28, 31. So ergeben sich mehrere Modellvorstellungen: Neben dem von Hwang et al. vorgeschlagenen Modell kann auch eine Polarisation des Korngrenzenbereichs durch angrenzende, magnetisch weiche Körner den Transport erklären [137|. Guinea stellt die Bedeutung von paramagnetischen Defektzuständen auf den Transport heraus [138], was von Ziese [139] aufgriffen wurde. Hiernach erfolgt der Transport durch spinpolarisiertes, inelastisches Tunneln durch eine spinglasartige Barriere. Philipp et al. kommen mittels einer systematischen niederfrequenten $1 / f$ Rauschanalyse zu einem ähnlichen Ergebnis [136]: Sie schlagen ein mehrstufiges inelastisches Tunneln über magnetische Defektzustände in einer spinglasartigen Barriere vor und betonen die Bedeutung einer möglichen Bandverbiegung unterhalb der Curie-Temperatur, welche zu einer Verarmungszone an der Korngrenze führt 28, 140.

Die obigen Betrachtungen zeigen die Schwierigkeiten bei der Identifizierung und Charakterisierung des Transportmechanismus auf, wobei aber besonders die niederfrequente $1 / f$-Rauschspektroskopie eine leistungsfähige Methode zu sein verspricht. Eine Möglichkeit zur Beseitigung dieser Interpretationsschwierigkeiten bietet die Entkopplung der einzelnen Körner durch eine zweite, isolierende Phase. Die Machbarkeit wurde für polykristalline Materialien bereits in Kapitel 2.5.2 vorgestellt. Die zweite Phase ändert dabei die 
Barrierenhöhe und so den Transportmechanismus. Häufig kommt es bei einem kritischen Volumenanteil der isolierenden Phase zu einem Perkolationsübergang. Tatsächlich lässt sich auch die Größe des Magnetowiderstands durch die Modifizierung der Korngrenzen beeinflussen: So beobachten Gupta et al. [66] und auch Balcells et al. [30] einen maximalen Magnetowiderstand in der Nähe der Perkolationsschwelle. Dennoch ist auch für diese Systeme der Transportmechanismus nicht endgülig geklärt, da wie in den konventionellen Dreilagensystemen die Grenzflächen Ferromagnet/Isolator von besonderem Interesse sind. Insofern kann argumentiert werden, dass es hier nur zu einer Verlagerung des Problems der reinen Korngrenzen-Systeme auf den Bereich der Grenzfläche Manganat/Isolator kommt. Yamada et al. 141 haben aber gezeigt, dass die Eigenschaften des Manganats an der Grenzfläche über die Barriere beeinflusst werden. So führt eine STO-Schicht auf LSMO wahrscheinlich aufgrund seiner Eigenschaften als Loch-Donator zu einer magnetisch toten Lage im LSMO. Über das gezielte Einbringen einer $\mathrm{LaMnO}_{3}$-Schicht konnte die magnetisch tote Lage an der Grenzfläche zum STO verhindert werden. Die Eigenschaften des Manganat an der Grenzfläche zum Isolator hängen somit vom isolierenden Material ab und können sich deutlich von den Charakteristika undotierter Korngrenzen unterscheiden. 



\section{Kapitel 4}

\section{Rauschen}

Üblicherweise wird das Rauschen in physikalischen Messgrößen als Störung angesehen. Eine genauere Betrachtung zeigt aber, dass aus den zeitlichen Fluktuationen zusätzliche Informationen über das zu untersuchende System gewonnen werden können. In der vorliegenden Arbeit ist das intrinsische, niederfrequente $1 / f$-Widerstandsrauschen von besonderem Interesse, welches nahezu jedem Festkörper zu Eigen ist. Diese Fluktuationen beeinhalten Informationen über die Dynamik von Defekten und können daher hervorragend zur zerstörungsfreien Charakterisierung von Festkörpern verwendet werden [142]. So zeigen beispielsweise polykristalline Goldfilme ein Rauschen, welches dreimal größer ist als in einkristallinen Filmen 142.

Zunächst werden in diesem Kapitel die mathematischen Grundlagen des Rauschens erarbeitet und wichtige Größen wie die spektrale Rauschleistungsdichte eingeführt. Anschließend werden die in Festkörpern beobachteten Arten des Rauschens vorgestellt, wobei der Schwerpunkt auf das niederfrequente $1 / f$-Rauschen gelegt wird. Die Ausführungen halten sich dabei eng an den hervorragenden Abhandlungen von Dutta et al. [143], Weissman [144], Raychaudhuri [142] und Kogan [145], denen weitere Informationen entnommen werden können.

\subsection{Mathematische Beschreibung}

Als Rauschen wird die zeitliche Fluktuation einer physikalischen Größe $x$, also das Abweichen vom Mittelwert $\langle x(t)\rangle$, bezeichnet:

$$
\delta x(t)=x(t)-\langle x(t)\rangle
$$

Der zeitliche Mittelwert ergibt sich dabei aus

$$
\langle x(t)\rangle_{t}=\frac{1}{t_{m}} \int_{-t_{m} / 2}^{t_{m} / 2} x(t) d t
$$


Die Messzeit $t_{m}$ muss ausreichend groß gewählt werden, exakt gilt die Definition nur für ein unendliches $t_{m}$. Um die Größe $\delta x(t)$ zu quantifizieren, wird zunächst die Autokorrelationsfunktion $\psi_{x}\left(t_{1}, t_{2}\right)$ bestimmt:

$$
\psi_{x}\left(t_{1}, t_{2}\right)=\left\langle\delta x_{1} \delta x_{2}\right\rangle_{t}=\lim _{t_{m} \rightarrow \infty} \frac{1}{t_{m}} \int_{-t_{m} / 2}^{t_{m} / 2} \delta x\left(t_{1}+t\right) \delta x\left(t_{2}+t\right) d t
$$

Für stationäre Systeme ist diese nur von der Differenz $t_{1}-t_{2}$ abhängig, da kein Zeitpunkt ausgezeichnet ist (Invarianz des Systems unter Zeittranslationen). Für $t_{1}-t_{2}=0$ ergibt sich aus der Autokorrelationsfunktion die mittlere quadratische Abweichung, welches als Varianz $\left\langle(\delta x(t))^{2}\right\rangle$ bezeichnet wird. Die Fouriertransformierte $\widetilde{\psi}_{x}$ der Autokorrelationsfunktion $\psi_{x}\left(t_{1}, t_{2}\right)=\psi_{x}\left(t_{1}-t_{2}\right)=$ : $\psi_{x}(t)$ ist nach dem Wiener-KhintchineTheorem 146, 147 mit der spektralen Rauschleistungsdichte $S_{x}(f)$ verknüpft:

$$
S_{x}(f)=2 \widetilde{\psi}_{x}(f)=2 \int_{-\infty}^{\infty} \psi_{x}(t) \cos (2 \pi f t) d t
$$

Da Messungen über einen endlichen Zeitraum ausgeführt werden, wird die spektrale Rauschleistungsdichte $S_{x}(f)$ über

$$
S_{x}(f)=2 \frac{1}{t_{m}}\left|\int_{-t_{m} / 2}^{t_{m} / 2} \delta x(t) e^{2 \pi i f t} d t\right|^{2}
$$

bestimmt. Dadurch können nur Frequenzen $f$ und Frequenzunterschiede $\Delta f$ größer $1 / t_{m}$ detektiert werden. Für die Varianz und die spektrale Rauschleistungsdichte ergibt sich folgender Zusammenhang:

$$
\left\langle(\delta x(t))^{2}\right\rangle=\int_{0}^{\infty} S_{x}(f) d f
$$

Das Integral der spektralen Rauschleistungsdichte über alle Frequenzen ist gleich der Varianz des Rauschens. Per Definition beschreibt die spektrale Rauschleistungsdichte also das Rauschen der Größe $x$ im Frequenzraum. Ist sie frequenzunabhängig, so wird auch von weißem Rauschen gesprochen, bei frequenzabhängigen Rauschen hingegen von farbigem Rauschen.

\subsection{Thermisches Rauschen}

Das so genannte thermische Rauschen (auch Johnson- oder Nyquist-Rauschen) wird in jedem dissipativen Widerstand durch die thermische Bewegung der Ladungsträger verur- 
sacht. Solange $k_{B} T \gg h f$ ist, gilt für die spektrale Leistungsdichte:

$$
S_{U}(f)=4 k_{B} T R
$$

Die Einschränkung $k_{B} T \gg h f$ kann aufgehoben werden, wenn Quantenkorrekturen hinzugefügt werden. Diese sind aber nur im Mikrowellenbereich notwendig. Das thermische Rauschen ist sowohl spannungs- als auch frequenzunabhängig. Es bildet damit einen konstanten Untergrund in Widerstandsrauschmessungen, der unter Umständen zur genauen Bestimmung eines Widerstandes verwendet werden kann.

\subsection{Schrotrauschen}

Das Schrotrauschen (engl.: shot noise) wird durch die diskrete Verteilung von Energie auf Teilchen verursacht, z.B. auf Elektronen in elektronischen oder Photonen in optischen Systemen. So setzt sich beispielsweise der Strom in einer Vakuumröhre aus unkorrelierten Emissionen von Elektronen aus der Kathode zusammen. Schottky konnte zeigen [148], dass die spektrale Rauschleistungsdichte proportional zur Ladung des Teilchens, in diesem Falle also zur Elektronenladung e, und zum Strom ist:

$$
S_{I}=2 \cdot e \cdot I
$$

Schrotrauschen ist folglich ebenfalls frequenzunabhängig und bietet weiterhin die Möglichkeit, die Ladung des Elektrons zu bestimmen.

\section{$4.4 \quad 1 / f$-Widerstandsrauschen}

\subsubsection{Allgemeine Beschreibung}

Zusätzlich zu den frequenzunabhängigen Beiträgen des thermischen Rauschens und des Schrotrauschens wird in vielen Systemen im niederfrequenten Spektrum (üblicherweise $10^{-2}-10^{4} \mathrm{~Hz}$ ) ein Anstieg der spektralen Rauschleistungsdichte gefunden. Dieser Anteil des Rauschens ist über viele Größenordnungen proportional zu $1 / f^{\alpha}$, wobei $0,8 \leq \alpha \leq$ 1,4 ist. Dieser Anteil wird üblicherweise als $1 / f$-Rauschen bezeichnet ${ }^{(1)}$. Es wurde 1925 von J.B. Johnson bei Messungen der Strom-Fluktuationen von der Elektronenemission in Glühkathodenröhren gefunden [149] und im folgenden Jahr von Schottky durch zufällige, langsame Veränderungen der Kathodenoberfläche beschrieben [150].

Das Auftreten des $1 / f$-Rauschens in vielen unterschiedlichen Systemen führt zu der Frage, ob ein universeller Rauschmechanismus existiert. Diese Frage ist jedoch bis heute unbeantwortet, da kein universell gültiges Modell gefunden und experimentell bestätigt werden konnte, so dass als Ursache für das Auftreten von $1 / f$-Rauschen in unterschied-

\footnotetext{
${ }^{(1)}$ Dieses $1 / f$-Rauschen ist nicht auf Widerstandsrauschen beschränkt, sondern tritt in vielen Systemen auf, wie z.B. in der Biologie, der Medizin oder auch in der Musik.
} 
lichen Systemen allgemeine Eigenschaften niederfrequenter Kinetik angenommen werden können [145].

Die in Festkörpern gemessenen Spannungsfluktuationen sind auf Widerstandsfluktuationen zurückzuführen, welche durch das Anlegen eines Stroms $I$ erst sichtbar gemacht, nicht aber verursacht werden. Dies wird üblicherweise durch die proportionale Abhängigkeit der spektralen Spannungsrauschleistungsdichte $S_{U}(f)$ von $I^{2}$ begründet. Einen experimentellen Nachweis lieferten Voss und Clark [151], indem sie Fluktuationen im thermischen Rauschen als Nachweis für Fluktuationen im Widerstand nutzten, die stromlos gemessen werden konnten. Das Widerstandsrauschen ist also ein intrinsisches Phänomen.

\subsubsection{Hooge-Parametrisierung}

Oft wird der so genannte Hooge-Parameter $\gamma$ zum Vergleich verschiedener Rauschmessungen herangezogen. Im Jahr 1969 stellte Hooge fest, dass sich die Rauschleistungsdichte umgekehrt proportional zum Probenvolumen $\Omega$, zur Ladungsträgerkonzentration $n$ und zur Frequenz $f$ verhält [152|:

$$
\frac{S_{R}(f)}{R^{2}}=\frac{S_{U}(f)}{U^{2}}=\frac{\gamma}{\Omega \cdot n \cdot f}
$$

Dabei schien der Parameter $\gamma$ für unterschiedliche Materialien mit $2 \cdot 10^{-3}$ konstant zu sein. Spätere Messungen zeigen allerdings, dass dies nicht der Fall ist. Es wurden Werte für $\gamma$ zwischen $10^{-8}$ und $10^{6}$ gefunden. Weiterhin zeigt sich, dass der Parameter eine Temperaturabhängigkeit aufweist.

Dennoch kann der Hooge-Parameter genutzt werden, um einen Vergleich der $1 / f$ Rauschamplituden verschiedener Materialien zu ermöglichen, da $\gamma$ ein Maß für das auf den Widerstand normierten Widerstandsrauschen ist. In der vorliegenden Arbeit ist jedoch die Ladungsträgerkonzentration des untersuchten Materials unbekannt, weshalb der Parameter $a_{U}$ wie folgt verwendet werden soll:

$$
a_{U}=\frac{\gamma}{n}=S_{U}(f) \cdot \frac{\Omega \cdot f^{\alpha}}{U^{2}}
$$

Der Exponent $\alpha$ wird aus einer Anpassung der experimentellen Daten an eine zu $1 / f^{\alpha}$ proportionale Funktion gewonnen. Der Parameter $a_{U}$ ist demzufolge frequenzunabhängig.

\subsubsection{Modell für $1 / f$-Widerstandsrauschen}

Im einfachsten Fall lässt sich die $1 / f$-Frequenzabhängigkeit des Rauschens durch eine Überlagerung verschiedener Spektren unterschiedlicher Rauschquellen erklären. Die einfachste isolierte Rauschquelle ist ein Schaltprozess zwischen zwei unterschiedlichen Werten $x_{1}$ und $x_{2}$, wobei der Prozess durch die Relaxationszeit $\tau$ charakterisiert wird. Die Autokorrelationsfunktion fällt exponentiell mit der Zeit ab und ergibt sich zu

$$
\psi_{x}(t)=\left\langle(\delta x)^{2}\right\rangle \cdot \exp (-|t| / \tau)
$$




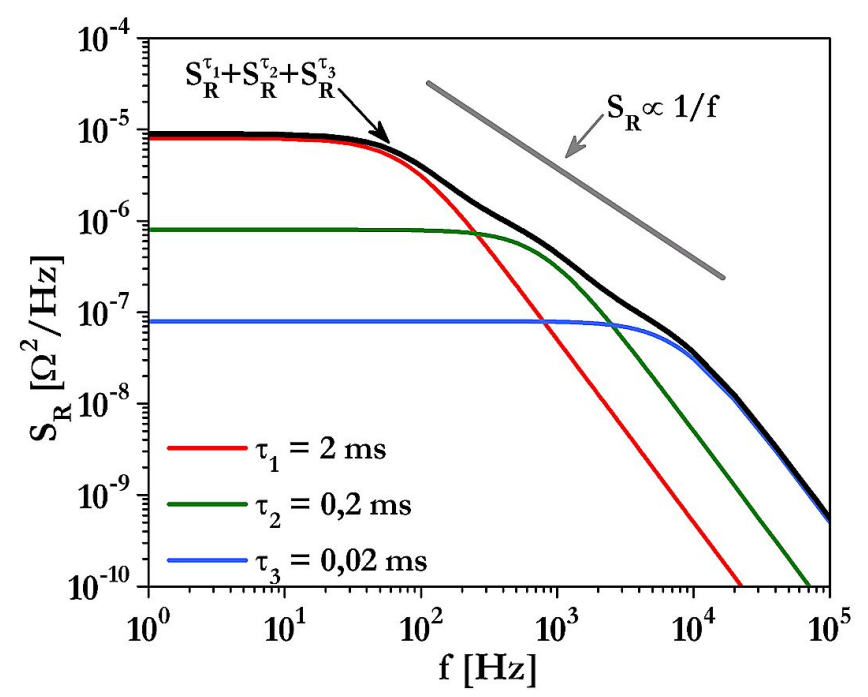

Abbildung 4.1. Spektrale Rauschleistungsdichte mehrerer Zwei-Niveau-Systeme unterschiedlicher Relaxationszeit. Die Überlagerung führt zu einem $1 / f$-artigen Spektrum.

Aus der Fouriertransformation der Autokorrelationsfunktion ergibt sich die spektrale Leistungsdichte dieses Rauschprozesses zu einem Debye-Lorentz-Spektrum:

$$
S_{x}(f)=\left\langle(\delta x)^{2}\right\rangle \cdot \frac{4 \tau}{1+(2 \pi f \tau)^{2}}
$$

Für Frequenzen sehr viel kleiner als $1 / \tau$ ist das Spektrum konstant, für höhere Frequenzen hingegen fällt das Spektrum mit $1 / f^{2}$ ab. Für niedrige Frequenzen, also große Zeiträume, finden viele Schaltvorgänge statt, so dass Anfangs- und Endzustand nicht mehr korreliert sind. Demnach ist keine Frequenzabhängigkeit zu beobachten. Für Frequenzen höher als $1 / \tau$ hingegen variiert $x$ mit zunehmender Frequenz weniger, die spektrale Rauschleistungsdichte nimmt ab.

Durch die Überlagerung verschiedener, voneinander unabhängiger Rauschquellen kann bei einer geeigneten Verteilung der Relaxationszeiten ein $1 / f$-Spektrum entstehen. Abbildung 4.1 zeigt die Spektren dreier einzelner Widerstands-Schaltvorgänge mit unterschiedlicher Relaxationszeit sowie deren Summe. Deutlich ist das Entstehen einer zu $1 / f$ proportionalen Abhängigkeit durch die Überlagerung zu erkennen. Die Verteilung $F(\tau)$ der Relaxationszeiten muss in der Bestimmung der Rauschleistungsdichte berücksichtigt werden:

$$
S_{x}(f) \propto \int_{0}^{\infty} d \tau F(\tau) \frac{4 \tau}{1+(2 \pi f \tau)^{2}}
$$

Hieraus folgt, dass eine Verteilung der Form $F(\tau) \propto \tau^{-\alpha}$ zu einem Potenzgesetz der Rauschleistungsdichte $S_{x} \propto f^{-2+\alpha}$ führt. Eine physikalische Motivation für $\alpha=1$ hätte demnach ein $1 / f$-Spektrum zur Folge 142.

Im Weiteren sei der für diese Arbeit interessante Fall des $1 / f$-Widerstandsrauschens betrachtet. In der Literatur existieren verschiedene Modelle, welche das $1 / f$-Rauschen erklären [142,145]. Für metallische Filme wird üblicherweise das Dimon-Dutta-Horn Mo- 
dell (DDH) angeführt [143], welches kurz erläutert werden soll. Ausgangspunkt ist eine Verteilung der Aktivierungsenergien $D(E, T)$ der Prozesse, die zu den Widerstandsfluktuationen führen. Dabei wird auch eine Temperaturabhängigkeit dieser Funktion zugelassen, wenn beispielsweise die Anzahl der Rauschquellen mit der Temperatur variiert. Die Aktivierungsenergie eines Prozesses steht dabei mit dessen Relaxationszeit über $\tau=\tau_{0} \cdot \exp \left(E / k_{B} T\right)$ in Beziehung, wobei $\tau_{0}^{-1}$ die Anklopffrequenz bezeichnet, mit der versucht wird, die Energiebarriere $E$ zu überwinden. Die spektrale Rauschleistungsdichte ergibt sich zu 145:

$$
\begin{array}{r}
S(f)=\int_{0}^{\infty} d E D(E, T) \frac{4 \tau_{0} \cdot e^{E / k_{B} T}}{1+\left(2 \pi f \tau_{0} \cdot e^{2 E / k_{B} T}\right)} \\
\quad=\frac{1}{\pi f} \int_{0}^{\infty} d E \frac{D(E, T)}{\cosh \left[\left(E-E_{\omega}\right) / k_{B} T\right]}
\end{array}
$$

Dabei wurde die Definition $E_{\omega}=k_{B} T \ln \left(2 \pi f \tau_{0}\right)^{-1}$ verwendet, womit $E_{\omega}$ im Bereich der Aktivierungsenergien liegt $(0,1-1 \mathrm{eV}) \mid 143.145$. Die Funktion $1 / \cosh \left[\left(E-E_{\omega}\right) / k_{B} T\right] \mathrm{im}$ Integranden von Gleichung 4.15 weist ein scharfes Maximum der Breite $k_{B} T$ um $E=E_{\omega}$ auf. Da die thermische Energie $k_{B} T$ auch für hohe Temperaturen klein ist gegenüber den Aktivierungsenergien, kann angenommen werden, dass die Breite der Verteilung der Aktivierungsenergien deutlich breiter ist als $k_{B} T$. Daher kann das Integral 4.15 berechnet werden:

$$
S(f)=k_{B} \cdot T \cdot D\left(E_{\omega}, T\right) \cdot \frac{1}{f}
$$

Da $E_{\omega}$ logarithmisch von $f$ abhängt, ergeben sich nur kleine Abweichungen von einer $1 / f$-Abhängigkeit für die spektrale Rauschleistungsdichte. Diese Abweichungen, welche sich im Exponenten $\alpha$ äußern, lassen sich folgendermaßen berechnen [143]:

$$
\alpha(f, T) \approx 1+\frac{1}{\ln \left(1 / 2 \pi f \tau_{0}\right)}\left\{\frac{\partial \ln S(f)}{\partial \ln T}-1\right\}
$$

Wird diese Temperaturabhängigkeit in Metallen gefunden, also das DDH-Modell bestätigt, so wird im Wesentlichen eine aktivierte, ausgeschmierte Kinetik identifiziert. Es ergeben sich aber in keiner Weise Informationen über die Natur der Defekte, die zu dieser Kinetik und dem gemessenen Spektrum führt. Daher wird das DDH-Modell auch als generisches, also allgemeines Modell bezeichnet.

Neben dem DDH-Modell, welches allgemein akzeptiert wird, existieren weitere Modelle, die ein $1 / f$-Verhalten erklären können. Auf diese Modelle soll im Folgenden nicht weiter eingegangen werden, ein Überblick findet sich in 145. 


\subsection{4 $1 / f$-Widerstandsrauschen in Manganaten und TMR-Ele- menten}

Aufgrund der Sensitivität des $1 / f$-Rauschens auf Defekte eignet sich diese als spektroskopische Methode zur Materialanalyse. Da sowohl in TMR-Systemen als in Manganaten Defekte eine große Rolle für den Ladungsträgertransport spielen, eignet sich hier die niederfrequente $1 / f$-Spektroskopie besonders. Für die Manganate konnte beispielsweise der Einfluss von Punktdefekten [153], Korngrenzen [134, 135, 136 und mechanischen Spannungen [154] auf den Ladungstransport besser verstanden werden. Auch die elektronische Phasenseparation (s. Kap. 2.3 155 sowie ein damit verbundenener Perkolationsübergang [156] konnte nachgewiesen werden. Somit können Rauschmessungen zum tieferen Verständnis der Manganate beitragen (s.a. [157, 158, 159, 160, 161]).

Auch für die TMR-Elemente (s. Kap. 3.2 kann die niederfrequente Rauschanalyse wichtige Beiträge zur Aufklärung des Ladungstransport liefern kann. Besonders nützlich erscheint die $1 / f$-Rauschspektroskopie zur Charakterisierung der Grenzflächen Ferromagnet/Isolator sowie der Barriere an sich. Wie in Kapitel 3.2 dargestellt, können dort befindliche Defekte den Magnetowiderstandseffekt beeinträchtigen. So konnte beispielsweise gezeigt werden, dass TMR-Elemente mit optimal präparierten Tunnelbarrieren ein deutlich geringeres Rauschen aufweist als mit Barrieren minderer Qualität [162, 163]. Die Rauschanalyse kann folglich genutzt werden, um TMR-Elemente zerstörungsfrei zu optimieren und zudem weitere Informationen über ihre Eigenschaften zu gewinnen [164,165. Weiterhin wird das Rauschen durch die thermisch aktivierte Bewegung von Domänenwänden beeinflusst und ist folglich oftmals magnetfeldabhängig [166, 167, 168]. Entsprechend kann der Einfluss von Domänenstrukturen auf den TMR charakterisiert werden. Zusätzlich ist das niederfrequente Rauschen im Rahmen von Anwendungen wichtig, stellt es doch eine limitierende Eigenschaft für die Verwendung eines Bauteils dar.

Die niederfrequente Rauschspektroskopie bietet demnach eine optimale Möglichkeit zur weiteren Charakterisierung von Manganat-basierenden TMR-Elementen. 



\section{Kapitel 5}

\section{Experimentelle Techniken}

\subsection{Probenpräparation}

\subsubsection{Metallorganische Aerosol-Deposition}

Alle in dieser Arbeit untersuchten Proben werden mittels Metallorganischer AerosolDeposition (MAD) hergestellt [169]. MAD ist eine chemische Herstellungsmethode, die ursprünglich für die Präparation von Hochtemperatursupraleiter-Schichten wie $\mathrm{YBa}_{2} \mathrm{Cu}_{3} \mathrm{O}_{7-\delta}$ entwickelt wurde [170]. Die Methode erwies sich auch als geeignet, um andere oxidische Schichten verschiedener Funktionalität herzustellen [171, 172]. Insbesondere die hier interessierenden Manganate lassen sich mit hoher Güte präparieren.

Ausgangspunkt für die Schichtpräparation sind verschiedene Metall-Chelat-Komplexe (Precursor) der in der Probe gewünschten Metallatome. In dieser Arbeit werden dazu ausschließlich Metall-Acetylacetonate verwendet, beispielsweise für das Mangan $\mathrm{Mn}\left(\mathrm{C}_{5} \mathrm{H}_{7} \mathrm{O}_{2}\right)_{3}$ (siehe Abbildung 5.1). Die Precursor werden der Zielzusammensetzung der Probe entsprechend in eine geeignete Lösung gebracht, wobei als Lösungsmittel eine flüchtige, organische Flüssigkeit, im speziellen Fall Dimethylformamid (DMF), dient. Durch Variation der Molverhältnisse kann so einfach die chemische Zusammensetzung der Probe geändert werden. Hierin liegt ein wesentlicher Vorteil gegenüber anderen Präparationsverfahren wie z.B. dem Sputterprozess, bei dem zur Änderung der Probenzusammensetzung ein neues Target herangezogen werden muss. Die Molarität der Lösung, also die Menge an Precursor pro Volumen Lösungsmittel, bietet einen weiteren Parameter in der Schichtpräparation, dessen Einfluss aber im Wesentlichen noch nicht verstanden ist. Für alle hier untersuchten Proben hat er keinen Einfluss auf die Qualität der Proben.

Die erhaltene Lösung wird mit Hilfe von trockener Druckluft bei einem Druck von ca. 2-3 atm durch eine Düse in den Reaktionsraum geleitet. Dadurch entsteht ein Aerosol mit einem Tröpfchendurchmesser von ca. 20-50 $\mathrm{m}$. Dieses Aerosol wird in Richtung des Substrats geleitet, welches seinerseits auf einem SiC-Heizer befestigt ist. Um einen steten Durchfluss zu erreichen, wird am unteren Ende des Reaktionsraumes das überschüssige Gas abgeleitet. Aufgrund der hohen Substrattemperatur verdampft das Lösungsmittel schnell und wird nicht deponiert. Unter optimalen Bedingungen sublimieren die übrigen Partikel direkt über dem Substrat. Über die Pyrolysereaktion (Gl. 5.1) kommt es zum 


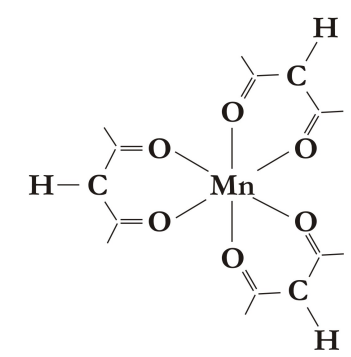

Abbildung 5.1. Mn-Acetylacetonat

Schichtwachstum.

$$
2 \mathrm{Mn}\left(\mathrm{C}_{5} \mathrm{H}_{7} \mathrm{O}_{2}\right)_{3}+36 \mathrm{O}_{2} \stackrel{\mathrm{T}_{0}}{\longrightarrow} \mathrm{Mn}_{2} \mathrm{O}_{3}+30 \mathrm{CO}_{2}+21 \mathrm{H}_{2} \mathrm{O}
$$

Die MAD-Technik bietet sowohl Vor- als auch Nachteile gegenüber anderen Präparationsverfahren. Um das Lösungsmittel zu verflüchtigen und die Pyrolysereaktion auszulösen, bedarf es thermischer Energie. Es existiert also eine minimale Depositionstemperatur. Die Präparation von Schichten bei Raumtemperatur ist daher ausgeschlossen, was Auswirkungen auf den Prozess der Mikrostrukturierung hat (1). Andererseits ist zur Deposition kein Vakuum nötig, so dass der apparative Aufwand deutlich reduziert wird. Durch die Verwendung von Druckluft wird insbesondere für die hier interessierenden Manganate auch die Gefahr des Sauerstoffdefizits stark reduziert.

\subsubsection{Probenherstellung}

In realiter werden einige Änderungen gegenüber der in Kapitel 5.1.1 beschriebenen Vorgehensweise vorgenommen. Diese betreffen insbesondere die Molverhältnisse der Precursor im Lösungsmittel. Aufgrund der stark unterschiedlichen Löslichkeiten der verschiedenen Acetylacetonate in DMF kommt es nicht zu einem exakten Übertrag der Mischungsverhältnisse auf die Probe. So muss zunächst empirisch ein optimales Mischungsverhältnis der Precursor gefunden werden, was beim hier interessierenden Manganat $\mathrm{La}_{0,7} \mathrm{Sr}_{0,3} \mathrm{MnO}_{3}$ über die Bestimmung der Curie-Temperatur erfolgt. In der optimalen Zusammensetzung ergibt sich die höchste Übergangstemperatur (siehe Abbildung 2.7).

Die Kontrolle der Depositionstemperatur erfolgt pyrometrisch. Hier ergeben sich Schwierigkeiten aufgrund durchsichtiger Substrate. So kann die gemessene Temperatur immer nur ein Richtwert für die Substrattemperatur sein. Die optimale Depositionstemperatur liegt dabei im Bereich von $700^{\circ} \mathrm{C}$ bis $950^{\circ} \mathrm{C}$.

Als Substrate kommen kommerziell erworbene, (001)-orientierte $\mathrm{Al}_{2} \mathrm{O}_{3}$-Einkristalle und (001)-orientierte MgO-Einkristalle zum Einsatz. Weiterhin werden auch so genannte Bikristalle aus $\mathrm{MgO}$ oder $\mathrm{SrTiO}_{3}$ verwendet. Diese bestehen aus zwei makroskopischen

\footnotetext{
${ }^{(1)}$ Eine Anwendung des Lift-off-Prozesses (siehe Kapitel 5.5 ist nicht möglich, da der Lack nur für Temperaturen von bis $\mathrm{zu} 100^{\circ} \mathrm{C}$ stabil ist.
} 


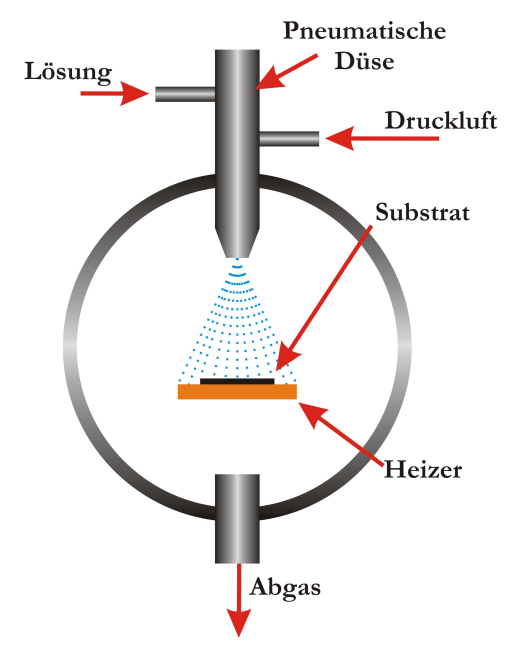

Abbildung 5.2. Schematischer Aufbau der MAD-Anlage.

einkristallinen Körnern mit einer definierten Korngrenze. Korngrenzen werden üblicherweise durch die Translation und Rotation der beteiligten Körner klassifiziert ${ }^{(2)}$. Für reine Rotationen wird üblicherweise zwischen tilt- und twist-Komponenten unterschieden (siehe Abb. 5.3). Ein tilt beschreibt die Rotation um eine Achse, welche in der Ebene der Korngrenze liegt, ein twist hingegen um eine senkrecht zu dieser. Eine $24^{\circ}$ [001]-tilt Korngrenze, wie sie die hier verwendeten Bikristalle aufweisen, verbindet demnach zwei Kristalle miteinander, welche um $24^{\circ}$ gegeneinander um die [001]-Richtung, welche beiden gemeinsam ist und in der Ebene der Korngrenze liegt, gedreht sind [Abbildung 5.3 (a)]. Eine Kombination von tilt- und twist-Komponenten führt zu so genannten gemischten Korngrenzen. Weiterhin wird zwischen symmetrischen und asymmetrischen Korngrenzen unterschieden. Korngrenzen mit identischen Missorientierungen der Körner in Bezug auf die Grenzfläche werden symmetrisch genannt, andernfalls asymmetrisch. In dieser Arbeit werden ausschließlich symmetrische Bikristalle verwendet.

\subsection{Röntgenstrukturanalyse}

Die Röntgenstrukturanalyse stellt eine zerstörungsfreie Standardmethode zur Bestimmung struktureller Eigenschaften dar. In der vorliegenden Arbeit werden zwei kommerzielle Röntgendiffraktometer in der Bragg-Brentano-Geometrie (auch $\theta$-2 $\theta$-Geometrie genannt, siehe Abbildung 5.4 verwendet. Dabei handelt es sich einerseits um ein D5000 der Firma Siemens, andererseits um ein D8 Advance der Firma Bruker AXS, wobei in beiden Geräten eine Kupferanode als Röntgenquelle dient. Mittels eines EinkristallMonochromators im Falle der D5000 bzw. eines Göbel-Spiegels bei der D8 Advance wird die $\mathrm{Cu}-\mathrm{K}_{\alpha}$-Strahlung herausgefiltert, welche sich in beiden Fällen als gewichtetes Mittel der $\mathrm{Cu}-\mathrm{K}_{\alpha, 1}$ und der $\mathrm{Cu}-\mathrm{K}_{\alpha, 2^{2}}$ Strahlung ergibt. Die Wellenlänge der resultierenden Strahlung beträgt $\lambda=1,54184 \AA$.

\footnotetext{
${ }^{(2)}$ Strenggenomen werden im dreidimensionalen Fall acht Parameter benötigt 174 .
} 
(a)

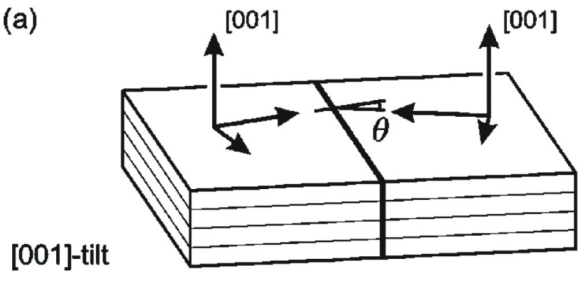

(b)

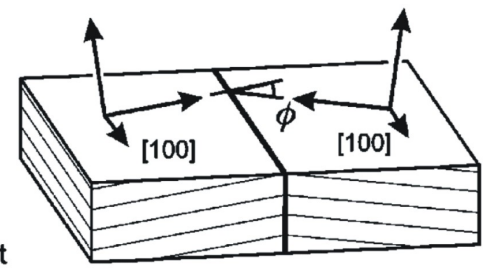

[100]-tilt

(c)

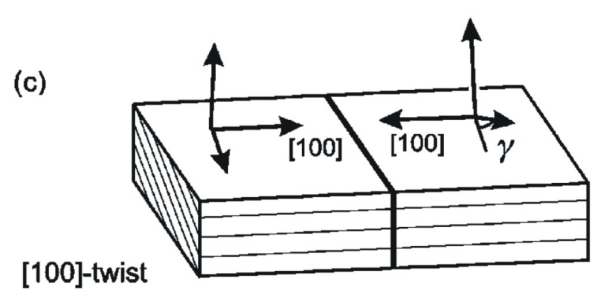

Abbildung 5.3. Schematische Darstellung der Kristallographie einer (a) [001]-tilt Korngrenze, (b) einer [100]-tilt Korngrenze und (c) einer [100]-twist Korngrenze in kubischen Materialien [173.

Da die eingestrahlten Röntgenquanten mit der Elektronenhülle der Atome wechselwirken, ist die am Detektor ermittelte Intensität mit der Elektronendichte $\rho_{\mathrm{e}}(\vec{r})$ des untersuchten Systems verknüpft 176:

$$
I(\vec{q}) \propto\left|\int \rho_{\mathrm{e}}(\vec{r}) e^{(i \vec{q} \cdot \vec{r})} d \vec{r}\right|^{2}=\left|\int f(\vec{q}) \rho(\vec{r}) e^{(i \vec{q} \cdot \vec{r})} d \vec{r}\right|^{2}
$$

Die am Detektor gemessene Intensität ist also proportional zum Betragsquadrat der Fourier-Transformierten der Elektronendichte. Durch die Einführung des Atomformfaktors $f(\vec{q})$, der das Streuvermögen eines einzelnen Atoms beschreibt, kann die Intensität in Abhängigkeit von der Atomdichte $\rho(\vec{r})$ dargestellt werden. Dabei bezeichnet $\vec{q}$ den Streuvektor, der sich aus

$$
\vec{q}=\vec{k}_{f}-\vec{k}_{i}
$$

ergibt, wobei $\vec{k}_{i}$ und $\vec{k}_{f}$ die Wellenvektoren des einfallenden bzw. reflektierten Strahls sind [176]. Aufgrund der gewählten Geometrie und der Annahme, dass es sich um elastische Streuung handelt (also $\left|\vec{k}_{i}\right|=\left|\vec{k}_{f}\right|=\frac{2 \pi}{\lambda}$ ), ist $\vec{q}$ senkrecht zur Probenoberfläche gerichtet mit $|\vec{q}|=\frac{4 \pi}{\lambda} \sin \theta$ (siehe Abbilung 5.4 rechts).

\subsubsection{Kleinwinkelanalyse}

Untersuchungen im Kleinwinkelbereich $\left(0,4^{\circ} \leq 2 \theta \leq 6^{\circ}\right)$ ermöglichen die Ermittlung der Schichtdicke sowie der Rauigkeit von Grenz- bzw. Oberflächen dünner Schichten. Die Beugung der Röntgenstrahlung erfolgt hier nicht wie bei der Weitwinkeluntersuchung am Kristallgitter, sondern an den Grenzflächen zweier Medien unterschiedlichen Brechungsindexes. Dies wird als optische Näherung bezeichnet, weshalb auch von Röntgenreflekto- 

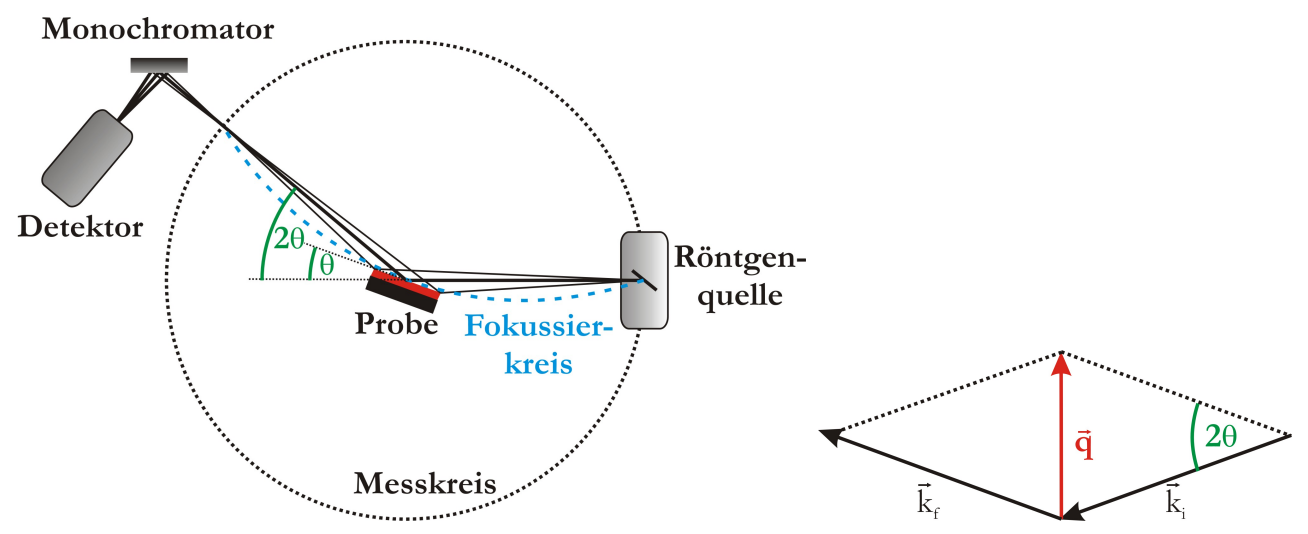

Abbildung 5.4. Bragg-Brentano-Geometrie. Nach [175.

metrie und nicht von Diffraktometrie gesprochen wird ${ }^{(3)}$,

Zur näheren Betrachtung wird der komplexe Brechungsindex herangezogen:

$$
n=1-\delta-i \beta
$$

Hierbei bezeichnet $\delta$ die Dispersion und $\beta$ die Absorption. Unterhalb des kritischen Winkels $\theta_{C}$ findet Totalreflexion statt, wobei $\theta_{C}$ mit der Dispersion $\delta$ zusammenhängt:

$$
\theta_{C} \approx \sin \theta_{C}=\sqrt{2 \delta}
$$

Die Interferenz der an unterschiedlichen Grenzflächen gebeugten Strahlen (z.B. die Grenzflächen Vakuum/Schicht und Schicht/Substrat) in Abhängigkeit des Streuvektors q führt zu charakteristischen Intensitätsmaxima bei $\theta_{i}(i=1,2,3, \ldots)$, welche folgendermaßen mit der Schichtdicke $d$ zusammenhängen [177:

$$
\theta_{\mathrm{i}}^{2}=2 \delta+\left(m_{\mathrm{i}}+\Delta m\right)^{2} \cdot \frac{\lambda^{2}}{4 d^{2}}
$$

Die Werte der einzelnen $m_{i}$ sind ganzzahlig, wobei $\Delta m$ den Wert 0 oder $\frac{1}{2}$ annimmt, je nachdem, ob an der Grenzfläche Schicht/Substrat eine Phasenschiebung um $\pi$ auftritt $(\Delta m=0)$ oder nicht $\left(\Delta m=\frac{1}{2}\right)$. Die Schichtdicke wird über die Bestimmung der Ordnung $m$ der Maxima mittels Minimierung des Fehlerquadrats nach Hink und Petzold [178 ermittelt.

${ }^{(3)}$ Die Vernachlässigung der Atompositionen und ein Auffassen eines Materials als Kontinuum für kleine Winkel (und somit kleine $q$-Werte) lässt sich durch Formel 5.2 verstehen: In der Näherung kleiner Winkel ist das Produkt (die Phase) $\vec{q} \cdot \vec{r}$ klein, weshalb die Atompositionen nicht mehr angegeben werden müssen. Ein weiteres Argument liegt in der Betrachtung der zugänglichen Abstände im Realraum, welche für kleine Winkel und $\mathrm{Cu}-\mathrm{K}_{\alpha}$-Strahlung im Bereich einiger Nanometer, also weit oberhalb interatomarer Abstände liegen. 


\subsubsection{Weitwinkelanalyse}

Weitwinkeluntersuchungen im Bereich $10^{\circ} \leq 2 \theta \leq 120^{\circ}$ machen aufgrund des geringeren Betrags des Streuvektors Strukturgrößen im Ångström-Bereich zugänglich. Es kann Aufschluss über Struktur, Gitterparameter oder auch Textur einer Probe gewonnen werden. Aufgrund der gewählten Bragg-Brentano-Geometrie sind jedoch nur Informationen entlang der Oberflächennormalen zugänglich.

Da in dieser Arbeit nur kristalline Materialien untersucht werden, soll an dieser Stelle die Beschreibung der Weitwinkeluntersuchungen auf diese eingeschränkt werden ${ }^{(4)}$. Für einen Kristall mit den Gittervektoren $\vec{a}_{1}, \vec{a}_{2}$ und $\vec{a}_{3}$ lässt sich die Elektronendichte $\rho(r)$ darstellen als Summe der Elektronendichte der einzelnen Elementarzellen $\rho_{E}(r)$ :

$$
\rho(\vec{r})=\sum_{n=(0,0,0)}^{N=\left(N_{1}, N_{2}, N_{3}\right)} \rho_{E}\left(\vec{r}+\vec{R}_{n}\right) \quad \text { mit } \quad \vec{R}_{n}=n_{1} \vec{a}_{1}+n_{2} \vec{a}_{2}+n_{3} \vec{a}_{3}
$$

Dabei bezeichnen die einzelnen $n_{i}$ die Anzahl der Elementarzellen in Richtung $\vec{a}_{i}$. Mit Hilfe dieses Ausdrucks kann Gleichung 5.2 vereinfacht werden zu:

$$
I(\vec{q}) \propto|F(\vec{q})|^{2} \cdot\left|\sum_{n}^{N} e^{-i \vec{q} \cdot \vec{R}_{n}}\right|^{2}
$$

Der so genannte Strukturfaktor $F(\vec{q})$ stellt dabei die Fouriertransformierte der Elektronendichte einer Elementarzelle dar. Aus ihm folgen die so genannten Auslöschungsregeln für bestimmte Gittertypen ${ }^{(5)}$. Gleichung 5.8 besagt, dass die Intensität maximal wird, falls der Streuvektor die Bragg-Bedingung erfüllt: $q_{\text {Bragg }}=2 \pi n / a, n=1,2,3, \ldots$ Dies ist äquivalent zu der Forderung, dass $\vec{q}$ mit einem Vektor des reziproken Gitters übereinstimmt:

$$
\vec{q}_{\text {Bragg }}=2 \pi h \frac{\vec{a}_{2} \times \vec{a}_{3}}{\vec{a}_{1} \cdot\left(\vec{a}_{2} \times \vec{a}_{3}\right)}+2 \pi k \frac{\vec{a}_{3} \times \vec{a}_{1}}{\vec{a}_{2} \cdot\left(\vec{a}_{3} \times \vec{a}_{1}\right)}+2 \pi l \frac{\vec{a}_{1} \times \vec{a}_{2}}{\vec{a}_{3} \cdot\left(\vec{a}_{1} \times \vec{a}_{2}\right)}, h, k, l \in \mathbb{Z}
$$

Die ganzen Zahlen $h, k$ und $l$ sind die Millerschen Indizes. Wird ein ermitteltes Röntgenspektrum zutreffend indiziert, werden also den Maxima die richtigen Millerschen Indizes zugeordnet, so kann neben einer sehr genauen Bestimmung des Gitterparameters zusätzlich auf die Orientierung streuender Bereiche in Bezug auf die Oberflächennormale rückgeschlossen werden. Werden beispielsweise nur Reflexe der Art (00l) beobachtet, so sind alle Körner derart orientiert, dass ihre (001)-Netzebenen parallel zur Oberfläche liegen. Allerdings kann nicht auf eine Epitaxie geschlossen werden, da eine Rotation der Körner um die $<001>$-Achse möglich ist, was aufgrund der eingeschränkten Sensitivität auf die Richtung der Oberflächennormale keinen Einfluss auf das Röntgenspektrum hat.

Aus der Halbwertsbreite $\Gamma$ eines Reflexes lässt sich weiterhin die Größe $\tau$ der kohärent

\footnotetext{
${ }^{(4)}$ Für die Untersuchung amorpher Materialien sei auf die Fachliteratur verwiesen, siehe z.B. [179] und 180 .

${ }^{(5)}$ Gleichung 5.8 kann noch weiter umgeschrieben werden, so dass die oft zu beobachtenden LaueOszillationen aus der Gleichung ersichtlich werden.
} 
streuenden Bereiche abschätzen. Sie ergibt sich aus der so genannten Scherrer-Formel 175]:

$$
\tau=\frac{0.9 \lambda}{\Gamma \cos \theta}
$$

Der Faktor 0.9 resultiert aus geometrischen Betrachtungen und gilt für kugelförmige Kristallite. Für eine Größenabschätzung ist dies aber ausreichend. Dabei ist wiederum zu beachten, dass nur die Größe in $q$-Richtung berechnet werden kann.

\subsection{SQUID-Magnetometrie}

Das SQUID-Magnetometer (engl.: S Superconducting Quantum Interference Device) stellt vermutlich die sensitivste Möglichkeit zur quantitativen Erfassung magnetischer Felder dar. In dieser Arbeit wird ein kommerzielles MPMS-5s-Magnetometer der Firma Quantum Design verwendet, welches die Bestimmung der Magnetisierung ${ }^{(6)}$ in einem Temperaturbereich von 1,8-400 K ermöglicht. Das System ist weiterhin mit einem supraleitenden Magneten ausgestattet, welcher ein maximales Magnetfeld von $50 \mathrm{kOe}$ erzeugt.

Das Herzstück zur Ermittlung der Magnetisierung besteht aus einem hochgradig ausbalancierten Gradiometer 2. Ordnung und einem rf-SQUID-System, welches einen extrem empfindlichen Magnetisierung-Spannungs-Konverter darstellt. Ein SQUID besteht aus einem supraleitenden Ring (meist eine dünne Schicht), welcher von einem (rf-SQUID) oder zwei (dc-SQUID) Josephson-Kontakten unterbrochen ist (siehe Abbildung 5.5). Es kombiniert zwei grundlegende Effekte, die auf der Phasenkohärenz im Supraleiter beruhen, nämlich die Flussquantisierung und den Josephson-Effekt.

Zunächst sei ein supraleitender Ring ohne Josephson-Kontakte betrachtet, durch dessen Fläche ein äußerer magnetischer Fluss $\Phi_{a}$ angelegt wird. Dieser Fluss kann beliebig sein, wobei aber festgestellt wird, dass der tatsächliche Gesamtfluss $\Phi$ durch den Ring aufgrund eines angeworfenen Kreisstroms $J$ immer nur ein Vielfaches des Flussquants $\Phi_{0}$ beträgt:

$$
\Phi=n \cdot \Phi_{0}, \quad \Phi_{0}=\frac{h}{2 e}=2,067 \cdot 10^{-15} \mathrm{~V} \cdot \mathrm{s}
$$

Der Ringstrom bewirkt also einen magnetischen Fluss $\Phi_{\text {ind }}=L J$ ( $L$ : Induktivität), so dass der Gesamtfluss gewissermaßen zum nächsten ganzzahligen Wert von $\Phi / \Phi_{0}$ auf bzw. abgerundet wird. Hierfür kann $L J$ maximal $\Phi_{0} / 2$ betragen.

Aufgrund der Phasenverschiebung an den Josephsonkontakten ändert sich durch deren Einfügen das Bild: Weiterhin gilt für den Gesamtfluss die Gleichung $\Phi=\Phi_{a}+L J$. Der Ringstrom $J$ fließst also durch den Josephsonkontakt, wodurch entsprechend der ersten Josephsongleichung $J$ mit dem kritischen Strom $I_{c}$ verknüpft wird [181]:

$$
J=I_{c} \sin \left(\varphi_{2}-\varphi_{1}\right)
$$

\footnotetext{
${ }^{(6)}$ Die magnetische Suszeptibilität ist ebenfalls zugänglich, wird aber im Rahmen dieser Arbeit nicht ermittelt.
} 


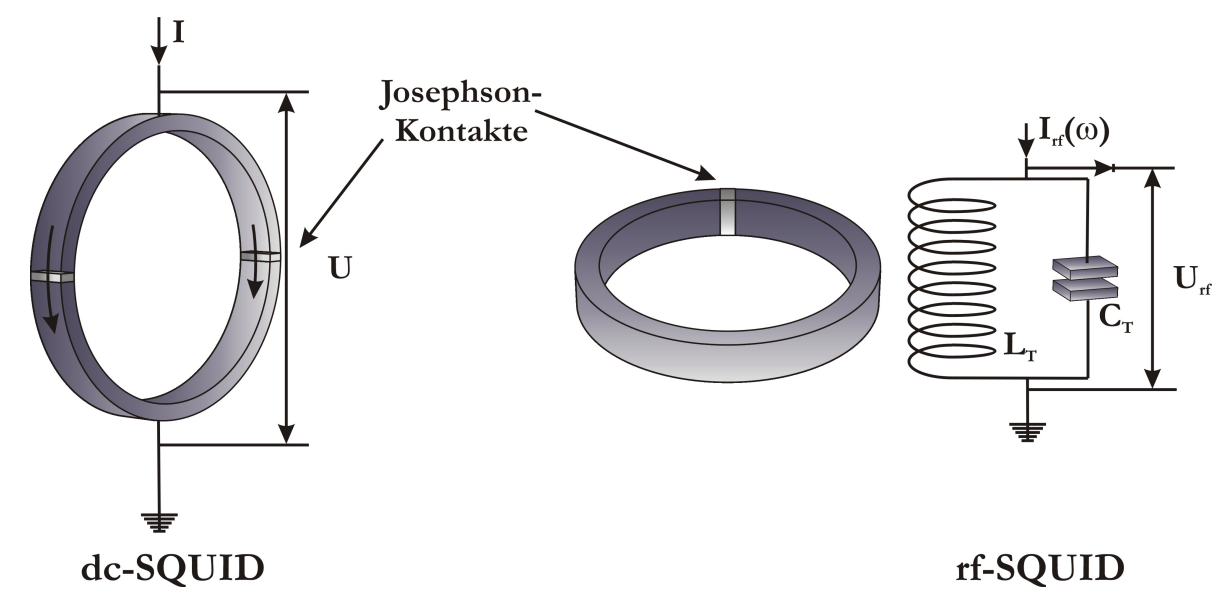

Abbildung 5.5. Schematische Darstellung eines dc-SQUIDs (links) mit zwei JosephsonKontakten und eines rf-SQUIDs (rechts) mit einem Josephson-Kontakt.

Hierbei bezeichnet $\varphi_{2}-\varphi_{1}$ die Phasendifferenz der Elektronen-Wellenfunktion über den Kontakt hinweg. Demnach ergibt sich der Gesamtfluss zu:

$$
\Phi=\Phi_{a}+L \cdot I_{c} \sin \left(\varphi_{2}-\varphi_{1}\right)
$$

Die Phasendifferenz kann folgendermaßen ausgedrückt werden [181:

$$
\left(\varphi_{2}-\varphi_{1}\right)=2 \pi\left(n-\frac{\Phi}{\Phi_{0}}\right)
$$

woraus folgt

$$
\sin \left(\varphi_{2}-\varphi_{1}\right)=-\sin \left(2 \pi \frac{\Phi}{\Phi_{0}}\right)
$$

und dadurch

$$
\Phi=\Phi_{a}+L \cdot I_{c} \sin \left(2 \pi \frac{\Phi}{\Phi_{0}}\right)
$$

Abbildung 5.6 zeigt graphisch den Zusammenhang zwischen $\Phi$ und $\Phi_{a}$ und macht deutlich, wie das SQUID den externen Fluss in Einheiten von $\Phi_{0}$ zählt. Entsprechend ist es möglich, jegliche Flussänderungen $\Delta \Phi_{a}\left(>\Phi_{0}\right)$ durch Messung der Spannungsvariation $\mathrm{U}(\Phi)$ an den Enden des Josephsonkontaktes zu detektieren.

Das SQUID bietet also eine sehr sensitive Möglichkeit zur Ermittlung magnetischer Dipole, welche von den Proben erzeugt werden. Dennoch wird in dem hier verwendeten Gerät eine andere Technik zur Bestimmung der magnetischen Eigenschaften angewandt. Statt die Probe direkt durch das SQUID zu bewegen, wird sie durch ein so genanntes Gradiometer zweiter Ordnung geführt (Abbildung 5.7). Dabei handelt es sich um einen supraleitenden Draht, welcher zu insgesamt vier Schleifen geformt wird. Die äußeren Beiden haben einen relativ großen Abstand zu den beiden Inneren, welche ihrerseits eine Spule mit zwei Wicklungen darstellen. Die Wicklungsrichtungen der beiden äußeren Windungen sind dabei gleich, aber denen der beiden Inneren entgegengesetzt. Auf diese Weise 


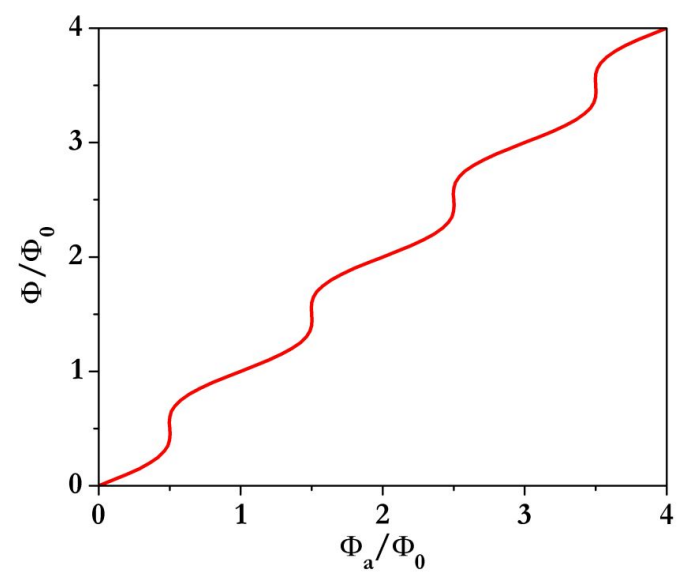

Abbildung 5.6. Gesamtfluss durch einen rf-SQUID Ring in Abhängigkeit vom äußeren Fluss.

haben Störquellen, welche oft deutlich weiter entfernt vom SQUID sind als die zu messende Quelle und daher ein in der Nähe des Interferometers nahezu konstantes Störsignal liefern, keinen Einfluss auf das Signal. Das magnetische Moment der Probe hingegen kann aufgrund der lokalen Änderungen des magnetischen Flusses im Gradiometer weiterhin bestimmt werden. Diese Anordnung ist nun mit einem rf-SQUID verbunden, welches aus einem SQUID besteht, das induktiv in einen elektrischen Schwingkreis eingekoppelt wird (Abbildung 5.5). Der große Vorteil dieser Methode liegt darin, dass das SQUID keinerlei elektrischen Kontakt zur Messelektronik hat. Der Schwingkreis erzeugt einen Wechselstrom, welcher einen variierenden Fluss induziert und zu einem Wechselstrom $I_{\mathrm{AC}} \mathrm{im}$ SQUID führt. Für den Fall $I_{\mathrm{AC}}>I_{c}$, welcher dann eintritt, wenn die Summe des $\mathrm{Su}-$ prastroms im Gradiometer und des Suprastroms des Schwingkreises groß genug ist, um eine Flussänderung von $\Phi_{0}$ im SQUID-Ring zu induzieren, dissipiert das Tunneln von Elektronen durch den Josephsonkontakt wie in einem Ohmschen Widerstand Energie und verursacht so eine Verminderung der abfallenden Spannung $U_{r f}$ des Schwingkreises, welche quantitativ ermittelt werden kann. Durch eine geeignete Eichung kann so mittels Bewegen durch das Gradiometer und Messen der Spannungsänderung am Schwingkreis das magnetische Moment einer Probe sehr genau bestimmt werden.

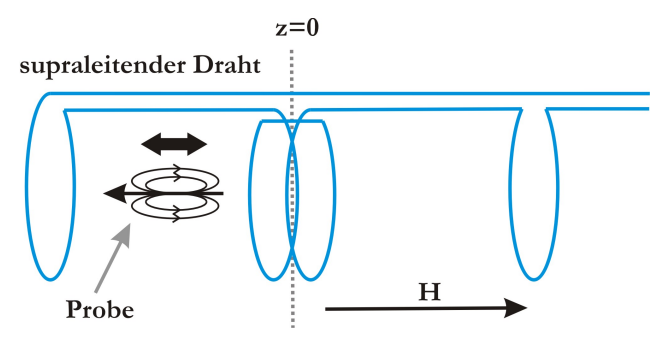

Abbildung 5.7. Aufbau eines Gradiometers zweiter Ordnung. 


\subsection{Raster-Elektronen-Mikroskopie}

Das Raster-Elektronen-Mikroskop (REM) bietet die Möglichkeit, die Oberfläche ausreichend leitfähiger Proben mit einem Auflösungsvermögen bis zu $5 \mathrm{~nm}$ abzubilden. In der vorliegenden Arbeit wird ein Supra 35 der Firma Leo verwendet, welches mit einer so genannten Gemini-Optik ausgestattet ist. Das REM nutzt die Tatsache, dass ein hochenergetischer Primär-Elektronenstrahl ( $E \leq 20 \mathrm{keV})$ Sekundärprodukte innerhalb eines so genannten Wechselwirkungsvolumens erzeugt. Werden diese Sekundärprodukte als Funktion des Ortes analysiert, was durch Rastern des Primärstrahls über die Probe realisiert wird, so können diese Informationen zur Bilderzeugung oder Materialanalyse genutzt werden.

Während die durch den Primärstrahl erzeugte Röntgenstrahlung mittels der energiedispersiven Röntgenspektroskopie (engl.: energy dispersive x-ray spectroscopy, EDX) weitgehend zur Materialanalyse genutzt wird, werden zur Bilderzeugung meist die Sekundär(SE) und Rückstreuelektronen (RE) verwendet. Sekundärelektronen werden durch inelastische Streuung der Primärelektronen am Atomkern oder an den Elektronen der Atomhülle des Probenmaterials erzeugt. Sie sind niederenergetisch $(<50 \mathrm{eV})$ und werden in unterschiedliche Gruppen eingeteilt, abhängig vom Entstehungsprozess und vom Bereich, in dem sie die Probe verlassen.

- SE1: diese Elektronen werden unmittelbar im Brennfleck des Primärstrahls erzeugt und verlassen auch dort die Probe.

- SE2: werden nach Mehrfachstreuung erzeugt und verlassen die Probe in größerem Abstand zum Brennfleck.

- SE3: entstehen durch Rückstreuelektronen weit entfernt vom Brennfleck und beinhalten keine Bildinformation.

Im Gegensatz zu den niederenergetischen Sekundärelektronen werden die hochenergetischen Elektronen $(\mathrm{E} \geq 50 \mathrm{eV})$, welche durch elastische Streuung in wesentlich tieferen Bereichen des Wechselwirkungsvolumens entstehen, als Rückstreuelektronen bezeichnet. Diese Elektronen tragen so zur Tiefeninformation bei. Zusätzlich ist aufgrund der hohen Abhängigkeit des Rückstreukoeffizienten von der Ordnungszahl des Materials auch ein Materialkontrast zugänglich. Zur Abbildung der Oberfläche sollten also möglichst die Sekundärelektronen der Typen 1 und 2 herangezogen werden, zur Materialkontrastgebung hingegen die Rückstreuelektronen.

Die hohe Auflösung des hier verwendeten Geräts ist insbesondere dem speziellen Strahlengang und dem darin befindlichen Inlens-Detektor zu verdanken. Diese Kombination bietet eine sehr effiziente Möglichkeit, das Signal auf SE1 und SE2 zu beschränken und auch mit niedrigen Beschleunigungsspannungen (also sehr oberflächensensitv) zu arbeiten. Für Beschleunigungsspannungen unterhalb $20 \mathrm{kV}$ erhalten die Primärelektronen in der Gemini-Optik im beam-booster eine zusätzliche Beschleunigung von $8 \mathrm{kV}$. Dadurch wird der Elektronenstrahl auch bei niedrigen Energien weniger anfällig für externe Störungen wie elektromagnetische Felder. Um letztlich die gewünschte Beschleunigungsspannung 


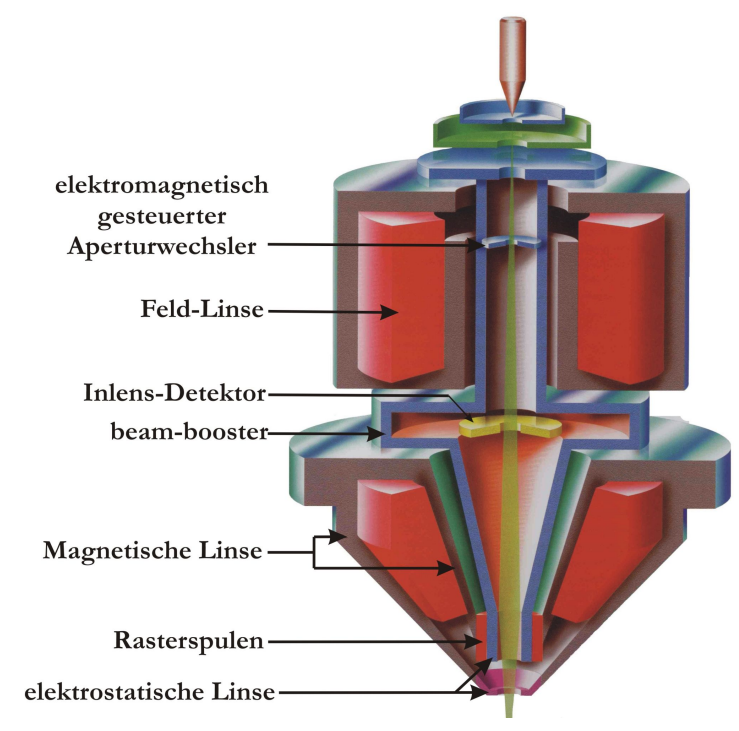

Abbildung 5.8. Schematischer Aufbau der Elektronenoptik des Leo Supra 35. Nach 182 .

der Primärelektronen zu erhalten, wird am Ende der Objektivlinse ein elektrostatisches Feld aufgebaut, welches die Primärelektronen um $8 \mathrm{kV}$ abbremst. Dieses Feld wirkt bei hinreichend kleinem Abstand zwischen Linse und Probe (Arbeitsabstand) zusätzlich auf die entstehenden SE, welche in den Strahlengang abgesaugt werden, in dem sich der Inlens-Detektor befindet. Die Intensität des erhaltenen Signals ist also mit dem anliegenden, elektrischen Feld verknüpft, dessen Stärke wiederum mit dem Abstand abnimmt. Dementsprechend empfiehlt sich, einen möglichst geringen Arbeitsabstand einzustellen.

Weiterhin beinhaltet das Gerät konventionelle SE2- sowie einen RS-Detektoren, welche beide ein geringeres Auflösungsvermögen aufweisen als der Inlens-Detektor. Mit Hilfe eines energiedispersiven Röntgenspektrometers, welches im hier verwendeten REM integriert ist, kann die Probenzusammensetzung quantitativ ermittelt werden. Ebenfalls integriert ist eine Lithographie-Einheit, welche separat vorgestellt wird.

\subsection{Mikrostrukturierung}

Um an den Proben definierte und auch reproduzierbare Widerstands- und Rauschmessungen vorzunehmen, wird eine Mikrostrukturierung etabliert. Hierbei stellen die Oxide aufgrund der hohen Depositionstemperatur eine Herausforderung dar, da sie erst nachträglich strukturiert werden können. Die Strukturierung erfolgt größtenteils durch Elektronenstrahl-Lithographie unter Verwendung eines Leo Supra 35 und der Lithographie Steuereinheit Elphy Plus der Firma Raith. Eine Nanokomposit-Proben wird mit optischen Methoden am Walther-Meissner-Institut, Garching strukturiert. Für manche Proben wird zudem eine weitere Reduzierung der Strukturgrößen durch einen fokussierten Ionenstrahl (engl.: focussed ion-beam, FIB) in Zusammenarbeit mit Hr. V. Radisch, Universität Göttingen, vorgenommen. 


\subsubsection{Von der Probe zur Struktur}

Da die Etablierung der Mikrostrukturierung mittels Elektronenstrahl-Lithographie einen wesentlichen Teil der vorliegenden Arbeit darstellt, soll der Weg einer Bikristall-Probe hin zur Mikrostruktur genauer beschrieben werden. Zunächst sollen die verwendeten Mikrostrukturen vorgestellt werden. Abbildung 5.9 zeigt zwei Strukturen, welche zur Ermittlung der resistiven Eigenschaften der Probe verwendet werden. Dabei bietet Struktur 1 den Vorteil, durch gleichzeitiges Messen des Widerstandes auf dem epitaktischen Teil der Probe (z.B. durch Verwendung der Spannungsabgriffe B und C) und über die Korngrenze hinweg (z.B. durch Verwendung der Spannungsabgriffe C und D) den Einfluss der Korngrenze sofort zu separieren. Die wesentlichen Kenngrößen dieser Mikrostruktur sind die Länge $l$ und die Breite $d$ der Brücke, welche durch zusätzlich mit Gold bedampfte Zuleitungen kontaktiert ist. Dabei ist $l=300 \mu \mathrm{m}$ und $d=50 \mu \mathrm{m}$. Die Gesamtstruktur hat eine Größe von $1 \times 1 \mathrm{~mm}^{2}$. Diese Gesamtgröße stellt die obere Grenze der Strukturgrößen dar, welche durch die minimale Vergrößerung des Elektronenmikroskops bedingt ist. Größere Strukturen oder auch Markierungen für eine zweite Struktur, welche über eine bereits existierende geschrieben werden soll, müssen infolgedessen durch ein Verfahren des Probentisches im Mikroskop realisiert werden. Hierdurch wird die Genauigkeit der Positionierung auf die Präzision des Tischmotors eingeschränkt, sie beträgt etwa $20 \mu \mathrm{m}$. Diese Ungenauigkeit limitiert auch die Positionierung der Strukturen auf der Korngrenze, da jene im lackierten Zustand im REM nicht zu erkennen ist. Demnach wird die Position der Korngrenze im unlackierten Zustand vom Rand der Probe bestimmt (ca. $5 \mathrm{~mm}$ ) und dieser Wert anschließend für die Belichtung verwendet. So muss in der Konzeption der gewählten Struktur ein Kompromiss gefunden werden zwischen einem vertretbar kleinen Abstand der inneren Spannungsabgriffe und einer möglichst geringen Fehlerquote in der Positionierung der Struktur über der Korngrenze.

Da für beide Strukturen zwei Belichtungsschritte notwendig sind (Ätzen der Struktur, Präparation der Goldkontakte mittels Lift-off-Technik), werden während des ersten Prozesses zusätzlich Marker positioniert, welche in definiertem Abstand zur eigentlichen Struktur ebenfalls in die Schicht geätzt werden. Sie dienen zur Orientierung beim zweiten Prozess. Aufgrund der Gesamtstrukturgröße wird die Positionierung aber über den Tischmotor realisiert, so dass Strukturbreiten unterhalb von $20 \mu \mathrm{m}$ nur unbefriedigend übereinander liegen.

Struktur 2 ist im Gegensatz zu Struktur 1 darauf ausgelegt, dass die Zuleitungen möglichst nah an die Korngrenze heranführen, um den Einfluss des epitaktischen Teils in der Messung zu minimieren. Aus diesem Grunde werden die Strom- und Spannungskontakte auf identischen Kontaktflächen angebracht. Weiterhin ist die Stegbreite mit $d \leq 5 \mu \mathrm{m}$ sehr klein gewählt, damit ein möglichst homogener Bereich der Korngrenze charakterisiert werden kann. Diese Struktur ist außerdem dafür vorgesehen, mittels FIB weiter ausgedünnt zu werden (siehe Kap. 5.5.2.

Aufgrund der hohen Depositionstemperaturen für Manganate (siehe 5.1) kommt ein Lift-off-Prozess nicht in Frage, so dass die Proben nachträglich strukturiert werden müssen. Nach der Reinigung wird ein Polymethylmethacrylat (PMMA)-Lack mit einer 


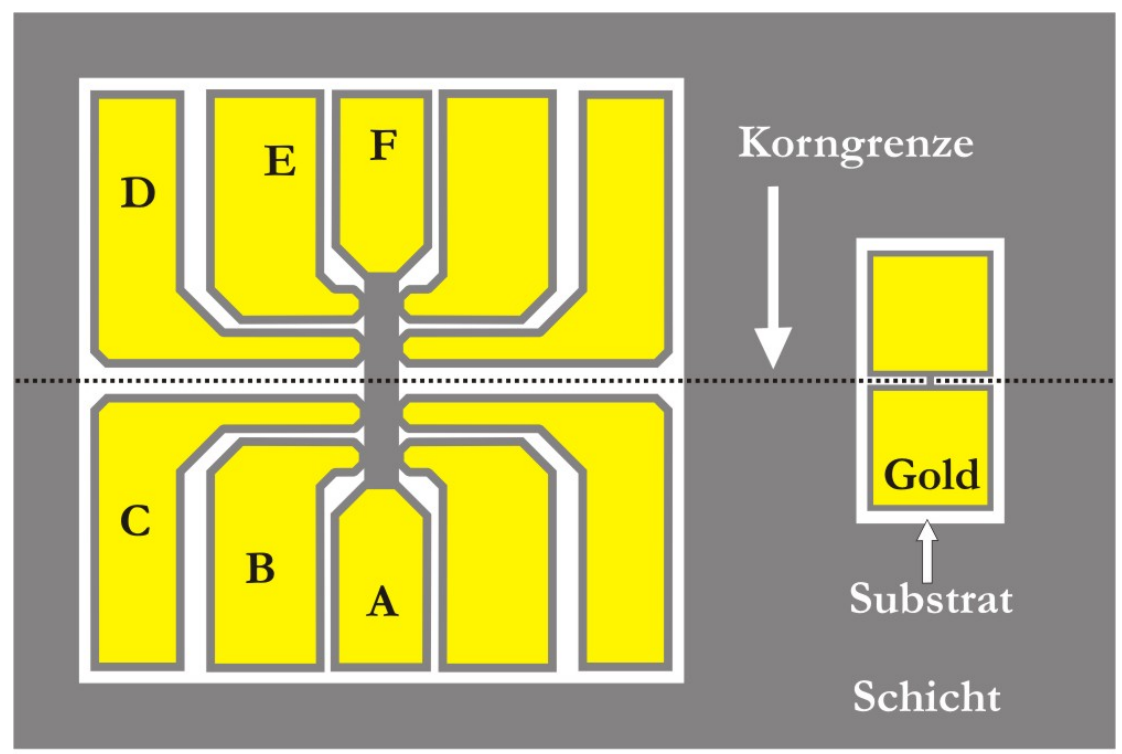

Struktur 1

Struktur 2

Abbildung 5.9. Schematische Darstellung der verwendeten Mikrostrukturen.

Schichtdicke von ungefähr $2 \mu \mathrm{m}$ auf der Probe aufgetragen. Hierzu wird der Positiv-Lack AR-P 671-09 der Firma Allresist verwendet und mit einer Umdrehungszahl von 2500 Umdrehungen pro Minute mittels eines Spin-coaters bei geschlossenem Deckel aufgetragen. Die vergleichsweise große Schichtdicke wird aufgrund des Feststoffgehalts von $9 \%$ in der aufgetragenen Lösung erreicht(7). Im Anschluss daran wird die Probe im Konvektionsofen für eine Stunde bei $170^{\circ} \mathrm{C}$ gehalten, was ein Glattziehen und Trocknen sowie ein Verdichten des PMMAs zur Folge hat. Nach Abkühlen der Probe kann die Belichtung der im Lack freizulegenden Stellen mit dem Elektronenstrahl erfolgen.

Durch die Bestrahlung des PMMAs werden bei geeigneter Dosis die Kettenbindungen des Polymers aufgebrochen, was eine höhere Löslichkeit in bestimmten organischen Lösungsmitteln zur Folge hat [z.B. das hier als Entwickler zum Einsatz kommende Methylisobutylketon (MIBK)]. Es handelt sich um einen positiv arbeitenden Lack, da die belichteten Stellen durch das Entwickeln entfernt werden. Wird eine deutlich höhere Dosis verwendet, so wirkt der Elektronenstrahl vernetzend auf die Polymerketten, so dass die Löslichkeit im Gegensatz zu unbelichteten Bereichen deutlich herabgesetzt wird. In diesem Falle wird auch von negativ arbeitenden Lacken gesprochen.

Für den hier verwendeten, positiv arbeitenden Lack $A R-P$ 671-09 und der verwendeten Schichtdicke von ca. $2 \mu \mathrm{m}$ wurde in Vorversuchen eine optimale Dosis von $181 \mu \mathrm{C} / \mathrm{cm}^{2}$ bei einer Beschleunigungsspannung von $20 \mathrm{kV}$ ermittelt. Bei Variation der PMMA-Schichtdicke durch Änderung der Rotationsgeschwindigkeit des Spin-coaters muss die Dosis entsprechend angepasst werden. Nach der Belichtung folgt die Freilegung der geschriebenen Strukturen durch Eintauchen der Probe in den Entwickler AR 600-56, ebenfalls hergestellt durch die Firma Allresist. In Vorversuchen ergab sich eine optimale

(7) Als Lösungsmittel dient in diesem Fall Chlorbenzol. 
Entwicklungszeit von 3 min.

Im nächsten Prozessschritt wird die in den Lack geschriebene Struktur auf die Schicht übertragen. Die Schicht soll also an den nicht bedeckten Stellen entfernt werden, an den bedeckten Stellen hingegen unbehandelt bleiben. Anfänglich wurde dieser Prozess durch nasschemisches Ätzen mittels $\mathrm{HCl}$ bzw. $\mathrm{HNO}_{3}$ vorgenommen, wobei der gesamte zugängliche Konzentrationsbereich ausgenutzt wurde. Dieser Prozess erwies sich jedoch als nicht praktikabel, da er zumeist zum Ablösen der gesamten Schicht führte oder nicht reproduzierbar war. Genaue Gründe für das Scheitern dieser Methode sind unbekannt. Denkbar ist ein Aufquellen des Lackes und eine damit verbundene Volumenaufweitung, welche durch mechanische Spannungen zum Ablösen von der Probe führt.

Eine andere Art, die Oberfläche kontrolliert abzutragen, besteht im so genannten Trockenätzen. Dieses Verfahren basiert im Gegensatz zum oben beschriebenen Prozess nicht auf einer chemischen, sondern einer physikalischen Wechselwirkung. Die Probe wird einem hochenergetischen $\mathrm{Ar}^{+}$-Strahl ausgesetzt $(\mathrm{E}=700 \mathrm{eV})$, wobei durch Impulsübertrag Atome aus oberflächennahen Lagen abgetragen werden. Das Verhältnis der Anzahl der abgetragenen Probenatome zu der auf die Oberfläche treffenden Ar-Ionen (engl.: sputter yield) ist aber von vielen Parametern abhängig und keineswegs für alle Materialien identisch. Der Tatsache, dass die hier zu bearbeitenden Manganate im Gegensatz zur PMMA-Schutzschicht einen deutlich geringeren sputter yield aufweisen, wurde Rechnung getragen, indem die PMMA-Schichtdicke auf $2 \mu \mathrm{m}$ angehoben wurde. Hierdurch ergeben sich zwar Einschränkungen in der minimal auflösbaren Strukturbreite, aber auch Schichten mit Dicken im Bereich von $50 \mathrm{~nm}$ können problemlos geätzt werden. Der experimentelle Aufbau dazu befindet sich im Institut für Materialphysik der Universität Göttingen. Nach dem Ätzvorgang wird das restliche PMMA mittels Aceton entfernt und die Probe nochmals gereinigt.

Um die Proben auch reproduzierbar kontaktieren zu können, müssen die Kontaktflächen der in die Schicht geätzten Struktur noch definiert werden. Dazu wird unter Verwendung des so genannten Lift-off-Prozesses an bestimmten Stellen eine metallische Schicht aufgebracht. Im vorliegenden Fall wird eine ca. $90 \mathrm{~nm}$ dicke Goldschicht thermisch aufgedampft, wobei zuvor eine ca. 5-10 nm dicke Chromschicht zur Verbesserung der Haftung des Goldes aufgebracht wird. Zur Deposition beider Materialien kommt die kommerzielle Aufdampfanlage Auto 306 der Firma Edwards zum Einsatz. Um nur definierte Stellen mit dem Metall zu bedecken, wird vor der Deposition wiederum eine PMMA-Schicht aufgebracht. Für den Lift-off-Prozess wird dabei der positiv arbeitende Lack AR-P 679-02 der Firma Allresist mit ebenfalls 2500 Umdrehungen pro Minute aufgebracht und anschließend eine Stunde lang bei $170^{\circ} \mathrm{C}$ im Konvektionsofen verdichtet. Aufgrund des geringeren Feststoffgehalts im Gegensatz zum im ersten Prozessschritt verwendeten Lack ergibt sich hier nur eine Schichtdicke von ca. $80 \mathrm{~nm}$. Anschließend erfolgt wiederum die Belichtung mittels des Rasterelektronenmikroskops mit einer zuvor ermittelten optimalen Dosis von $188 \mu \mathrm{C} / \mathrm{cm}^{2}$ bei einer Beschleunigungsspannung von $20 \mathrm{kV}$. Dabei werden die Stellen belichtet, an denen die Kontaktschicht aufgebracht werden soll. Anschließend erfolgt die Entwicklung durch Eintauchen in den Entwickler $A R$ 600-56 (30 s) sowie anschließendes Stoppen der Entwicklung durch Eintauchen in Isopropanol (ebenfalls 30 s). Nun kann das 
Metallisieren der freigelegten Stellen erfolgen. Im Anschluss wird die Schicht für mehrere Stunden in Aceton gelegt, um das verbleibende PMMA abzulösen. So verbleibt nur an den zuvor freigelegten Stellen der Schicht das Gold als Kontaktfläche. Nicht vollständig gelöstes PMMA kann im Ultraschallbad bei geringster Leistungsstufe abgelöst werden.

\subsubsection{Minimierung der Strukturgrößen unter Verwendung eines fokussierten Ionenstrahls}

Die Kombination eines REMs mit einem fokussierten Ionenstrahl (engl.: focussed ion beam, FIB) bietet die Möglichkeit, die Strukturgröße des Stromstreifens über die Korngrenze hinweg deutlich zu verkleinern, wobei während des Materialabtrags durch den Ionenstrahl dieser zeitgleich unter Verwendung des Elektronenstrahls beobachtet werden kann. In dieser Arbeit kommt ein FEI xT Nova Nanolab 200 zum Einsatz. Dieses Gerät kombiniert ein konventionelles REM mit einer $\mathrm{Ga}^{+}$-Quelle, welche umgangssprachlich auch als „NanoLaubsäge" bezeichnet wird. Mit Hilfe des $\mathrm{Ga}^{+}$-Strahls, der auf einen Durchmesser von ca. $40 \mathrm{~nm}$ fokussiert werden kann, ist es möglich, Material definiert abzutragen oder auch zu deponieren. Der Abtrag funktioniert ähnlich wie beim Sputterprozess, wobei die Position des Ionenstrahls über ein Ablenksystem gesteuert werden kann. Zur definierten Deposition von Material wird ein geeignetes Carriergas in den Probenraum eingeleitet. Das zu deponierende Material (hier: $\mathrm{Pt}$ und $\mathrm{SiO}_{2}$ ) ist darin chemisch gebunden, der Elektronenoder Ionenstrahl bricht die Bindung auf, es kommt zur Deposition. Die Verwendung des Ionenstrahls bietet dabei zeitliche Vorteile, hingegen kann eine Beschädigung der unterliegenden Schicht nicht ausgeschlossen werden. Bei sensitiven Bereichen kommt daher die Deposition mittels Elektronenstrahl zum Einsatz, welche zeitlich deutlich aufwändiger, aber zerstörungsfrei ist.

In Abbildung 5.10 ist der Vorgang der Strukturierung mittels FIB schematisch dargestellt. Wie in Kapitel 5.5.1 erwähnt, dient die mittels Elektronenstrahl-Lithographie präparierte Struktur 2 als Ausgangspunkt. Nachdem die Korngrenze mit dem REM lokalisiert ist, wird sie mit einer isolierenden, mehrere $100 \mathrm{~nm}$ dicken $\mathrm{SiO}_{2}$-Schicht bedeckt, um sie vor Beschädigungen durch den Ionenstrahl oder Bedeckungen mit leitfähigen Materialien beim Materialabtrag, also vor elektrischen Kurzschlüssen, zu schützen. Die Deposition wird mittels Elektronenstrahl vorgenommen, um jegliche Beschädigungen der Korngrenze zu vermeiden. Anschließend werden Pt-Leiterbahnen von den Goldkontakten bis nahe an die $\mathrm{SiO}_{2}$-Schutzschicht heran deponiert, welche den Gesamtwiderstand des Kontaktes reduzieren sollen (siehe Abbildung 5.11, links). Nun kann die Struktur ausgedünnt werden. Hierzu wird mit dem fokussierten Ionenstrahl an der Korngrenze Material abgetragen, wobei ein Bereich von ca. 1,5 $\mu \mathrm{m}$ Breite gewählt wird, um den Stromstreifen sicher im Bereich der Korngrenze auszudünnen. Abbildung 5.11b) zeigt den Beginn des Abtrages an der unteren Seite des Stromstreifens. Durch symmetrischen Abtrag an der unteren und oberen Seite des Steges kann eine Ausdünnung des Kontaktes bis zu einer Breite von ca. $40 \mathrm{~nm}$ erreicht werden. Abbildungen 5.12 und 5.13 zeigen die fertige Struktur, wobei auch die Korngrenze und die zu den Goldkontaktflächen führenden Pt-Zuleitungen zu erkennen sind. 

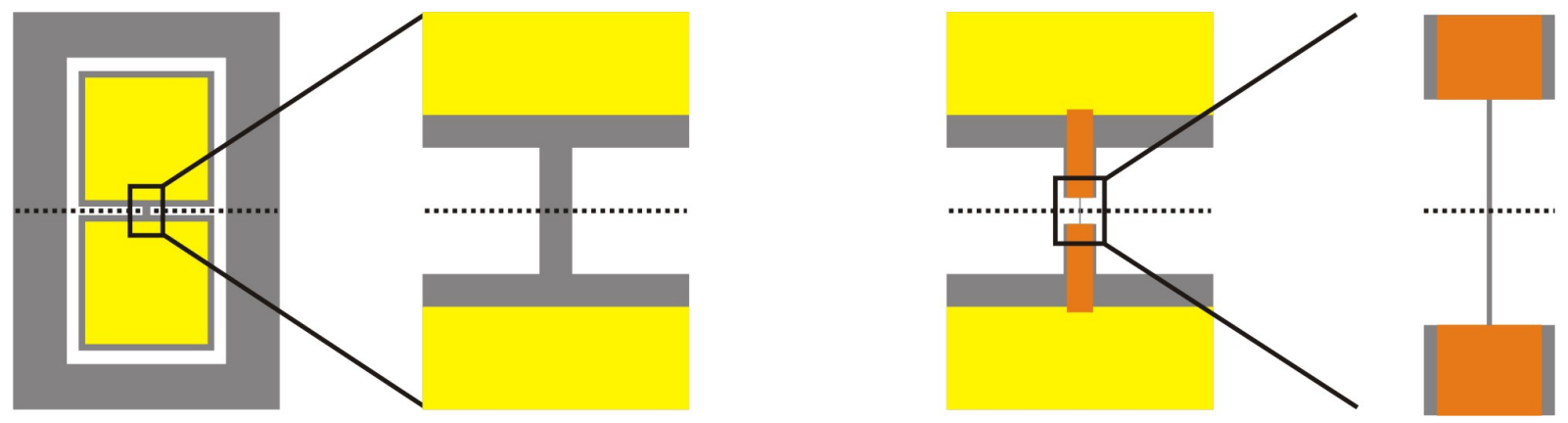

Abbildung 5.10. Schematische Darstellung der Vorgehensweise zur Minimierung der Strukturgröße unter Verwendung des FIBs. Links: Ausgangspunkt ist die in Kapitel 5.5.1 beschriebene Struktur 2. Rechts: Pt-Zuleitungen (orange) führen zur ausgedünnten Stelle des Stromstreifens, welche die Korngrenze überquert.
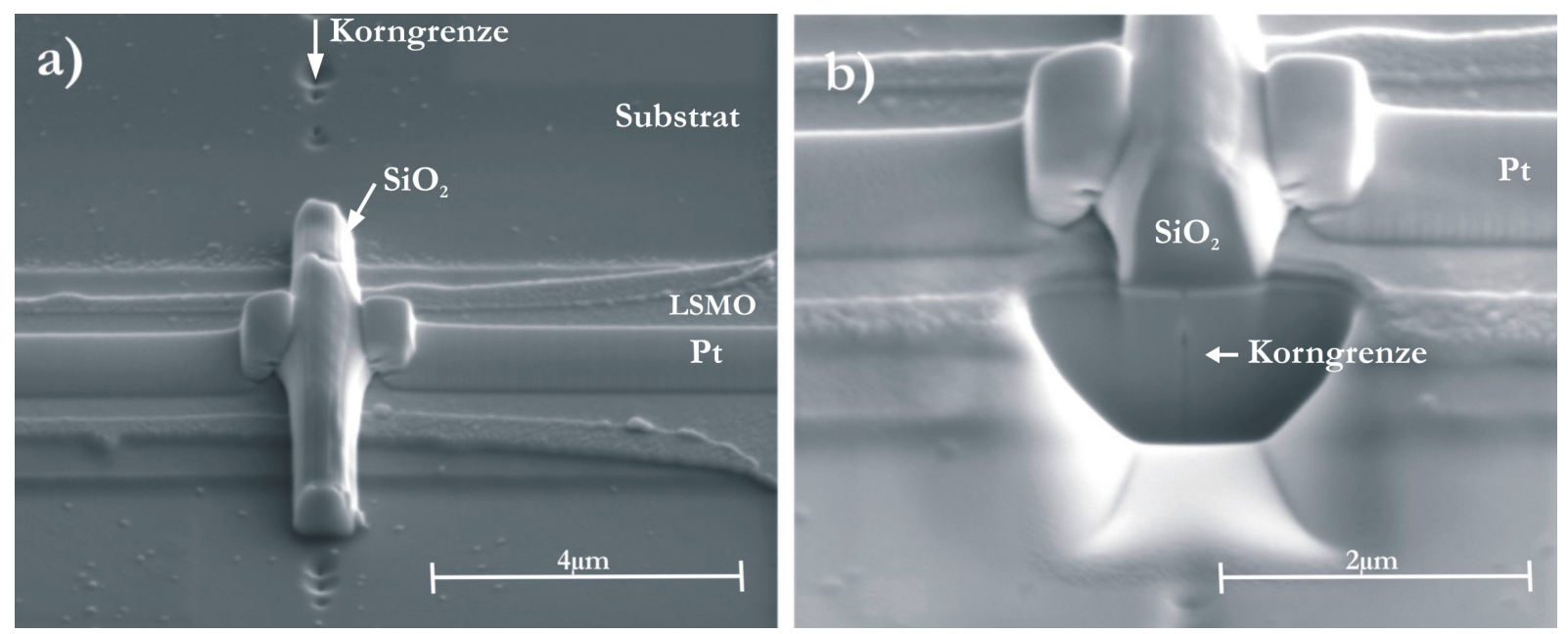

Abbildung 5.11. a) REM-Aufnahme des Stromstreifens über die Korngrenze vor der Verwendung des Ionenstrahls. Die Korngrenze wurde mit isolierendem $\mathrm{SiO}_{2}$ abgedeckt, wobei zur Deposition der Elektronenstrahl zum Einsatz kam, um etwaige Beschädigungen der Korngrenze durch den Ionenstrahl zu verhindern. Weiterhin sind die bereits aufgebrachten Platinzuleitungen zu erkennen. b) Beginn des Materialabtrages an der unteren Kante des Stromstreifens. Zu erkennen ist die durch das Substrat laufende Korngrenze. 


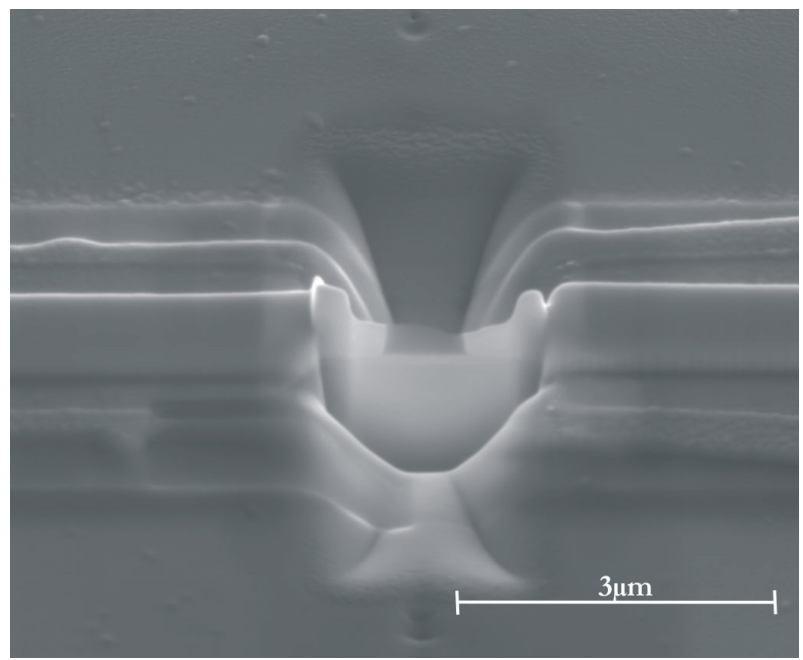

Abbildung 5.12. REM-Aufnahme des mittels FIB ausgedünnten Kontaktes. Zu erkennen ist das die Korngrenze schützende $\mathrm{SiO}_{2}$, welches hier dunkel erscheint. Es verhindert auch einen Kurzschluss der Pt-Zuleitungen.

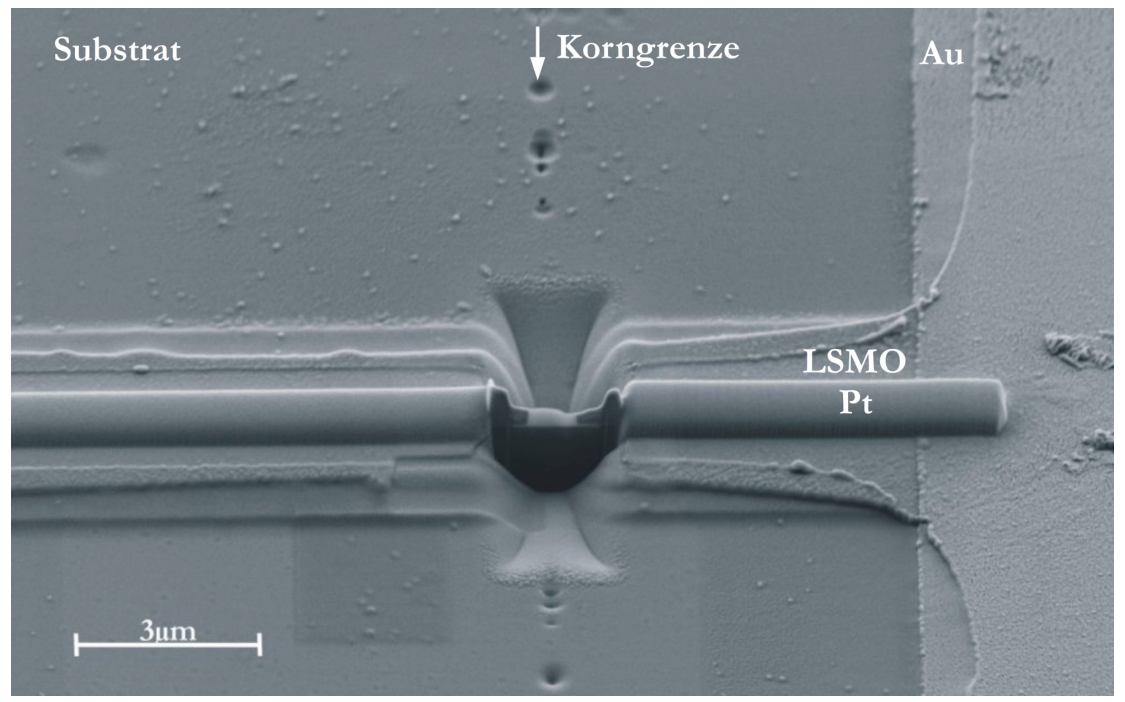

Abbildung 5.13. REM-Aufnahme des mittels FIB ausgedünnten Kontaktes. Es zeigt sich, dass die Korngrenze, welche auf dem freigelegten Substrat besser zu erkennen ist, durch die ausgedünnte Stelle des Stromstreifens verläuft. Ebenfalls zu erkennen: die direkte Verbindung zwischen den Goldkontakten und den Pt-Zuleitungen. 


\subsection{Transmissions-Elektronen-Mikroskopie}

Das Transmissions-Elektronen-Mikroskop (TEM) bietet ebenso wie das REM eine Methode zur direkten Bildgebung, allerdings wird die Probe durchstrahlt und daher nicht die Oberfläche abgebildet. Dieser Zugang erlaubt (zusammen mit der weit höheren Beschleunigungsspannung und der damit verbundenen reduzierten Wellenlänge der Elektronen) eine sehr hohe Auflösung und dadurch differenzierten Aufschluss über die Struktur einer Probe. Das TEM kann in verschiedenen Modi betrieben werden: (hochauflösender) Hell- und Dunkelfeldmodus, Beugungsmodus, Elektronen Energieverlust-Spektroskopie, Röntgen-Mikroanalyse und mehr. Für die vorliegende Arbeit wird das verwendete Philips CM200 bei einer Spannung von $200 \mathrm{kV}$ ausschließlich im Hellfeldmodus betrieben sowie zur Röntgen-Mikroanalyse verwendet. Aufgrund der Durchstrahlung der Probe muss diese sehr dünn sein (5-500 nm), was mittels des FIBs realisiert wird 77].

Ein TEM wird üblicherweise in die Beleuchtungs- und die Abbildungseinheit unterteilt. In der Beleuchtungseinheit werden die Elektronen erzeugt und auf die Probe fokussiert, während in der Abbildungseinheit aus den von der Probe gebeugten Elektronen ein Bild auf dem Schirm erzeugt wird. Abbildung 5.14 zeigt den Strahlengang der Abbildungseinheit für den vereinfachten Fall, dass neben den Linsen der Beleuchtungseinheit nur eine Objektivlinse und eine Projektionslinse verwendet werden. Reale Systeme sind in der Regel noch mit mindestens einer weiteren Linse ausgestattet. Zunächst sei der allgemeine Bildgebungsmodus vorgestellt (Abbildung 5.14, links): Aufgrund der Wechselwirkungen mit der Probe werden die Elektronen gebeugt und treffen anschließend auf die Objektivlinse. In deren Brennebene entsteht das Beugungbild. Hier treffen sich Strahlen von allen Punkten der Probe, die unter identischen Winkeln gebeugt werden. Die Projektionslinse ist auf die Bildebene der Objektivlinse fokussiert und bildet das dort entstehende Zwischenbild auf dem Schirm ab. Dieses zeigt aber nur wenig Kontrast, welcher durch den Wechsel in den Hell- bzw. Dunkelfeldmodus stark erhöht werden kann. Durch das Einfügen einer Objektivblende kann das Bild durch Strahlen erzeugt werden, die alle durch einen Punkt in der Brennebene verlaufen, welche also alle von der Probe in die gleiche Richtung gebeugt werden. Wird die Blende so positioniert, dass nur die ungebeugten Elektronen zum Bild beitragen (Abbildung 5.14. Mitte), so wird vom Hellfeldmodus gesprochen. Wird ein Bild hingegen von gebeugten Elektronen erzeugt (Abbildung 5.14, rechts), so wird es als Dunkelfeldaufnahme bezeichnet. Experimentell wird der Dunkelfeldmodus jedoch abweichend von der Darstellung meist durch ein Verkippen der Beleuchtungseineit realisiert, da aufgrund der sphärischen Aberration der Linsen diejenigen Strahlen bevorzugt werden sollten, welche nahe und parallel der optischen Achse verlaufen ${ }^{(8)}$,

Um detaillierte Informationen auf der Längenskala der Gitterkonstante zu erhalten, muss das TEM im hochaufösenden Modus betrieben werden. Da die gebeugte Elektronenwelle im Wesentlichen die Fouriertransformierte der Verteilung des Streufaktors ist, welche die Atomverteilung wiedergibt, wird im $k$-Raum eine gewisse Bandbreite $\delta k$ be-

\footnotetext{
${ }^{(8)}$ Abbildung 5.14 ist horizontal stark vergrößert dargestellt, die Beugungswinkel liegen typischerweise unter einem Grad.
} 

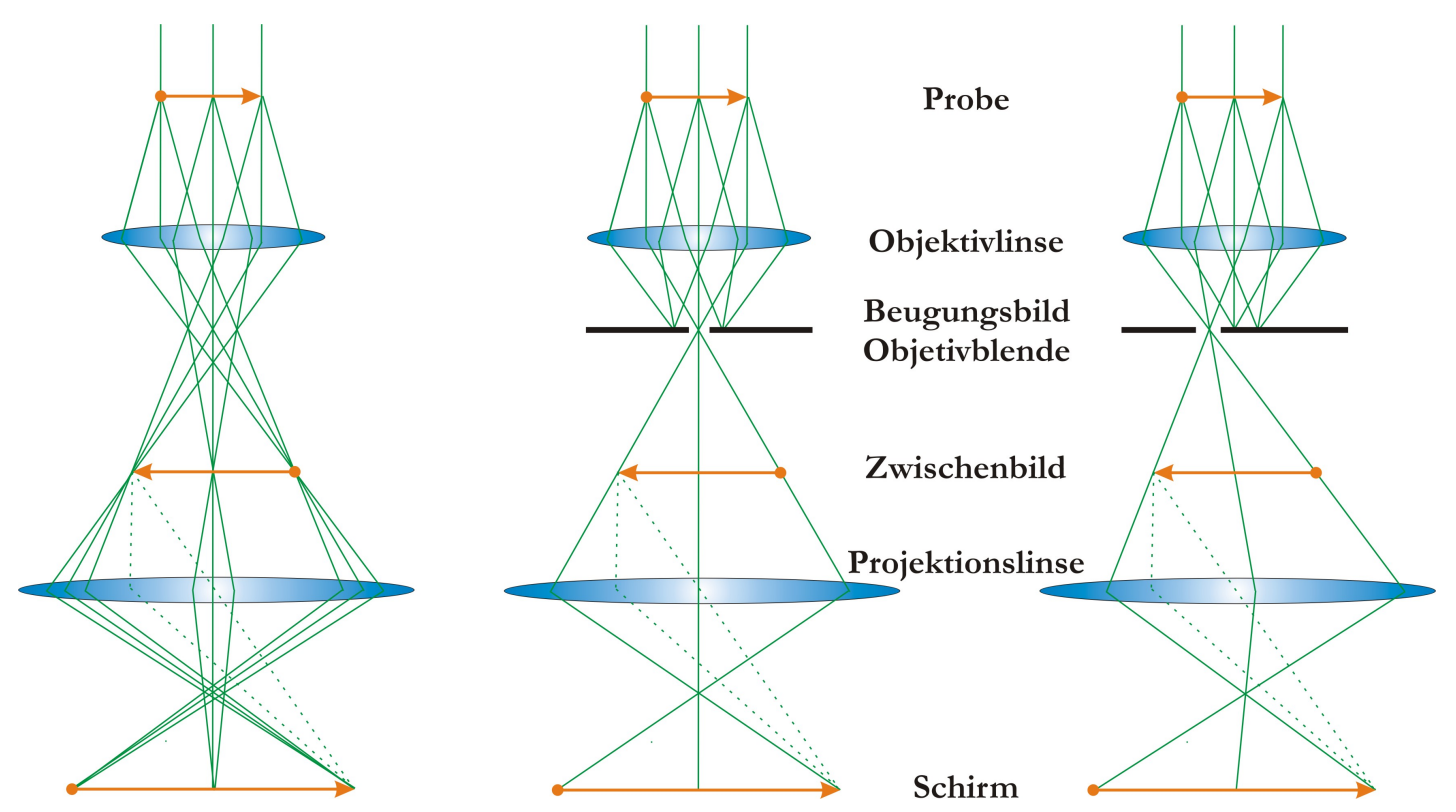

Abbildung 5.14. Strahlengang des TEMs im bildgebenden Modus (links), Hellfeldmodus (Mitte) und Dunkelfeldmodus (rechts). Nach [183].

nötigt, um eine Auflösung von $\Delta x \approx 2 \pi / \delta k$ zu erlangen ${ }^{(9)}$. Hieraus folgt direkt, dass im Hell- und Dunkelfeldmodus keine Hochauflösung erreicht wird. Diese entsteht erst durch die Interferenz des Nullstrahls mit Strahlen höherer Ordnung. Da die höheren Ordnungen aber weiter entfernt und nicht so parallel zur optischen Achse verlaufen, werden hohe Anforderungen an das Linsensystem gestellt.

Neben der direkten Abbildung der Probe kann das TEM auch zur Mikroanalyse genutzt werden. Der Elektronenstrahl führt u.a. zu Anregungen der Probenatome, was in der Emission von charakteristischer Röntgenstrahlung resultiert. Die Intensität einer charakteristischen Energie eines Atoms ist zunächst ein Hinweis auf die Existenz des betreffenden Elements in der Probe, also zur qualitativen Analyse nützlich. Weiterhin kann mit Hilfe von Korrekturfaktoren auf die quantitative Zusammensetzung geschlossen werden, worauf in dieser Arbeit verzichtet wird (siehe hierzu z.B. [183]). Unter der Verwendung eines fein fokussierten Elektronenstrahls, der über die Probe gerastert wird, sind so auch ortsaufgelöste Informationen über die Probenzusammensetzung zugänglich. Eine solche Mikroanalyse wird auch als Nano-EDX bezeichnet.

Alle in dieser Arbeit präsentierten TEM-Untersuchungen werden in Zusammenarbeit mit PD M. Seibt und Dr. K. Thiel, IV. Physikalisches Institut der Universität Göttingen, vorgenommen.

\footnotetext{
${ }^{(9)}$ Ein Bild, welches aus einem kleinen Intervall von $k$-Werten aufgebaut wird, kann nur langreichweitige, räumliche Merkmale widergeben.
} 


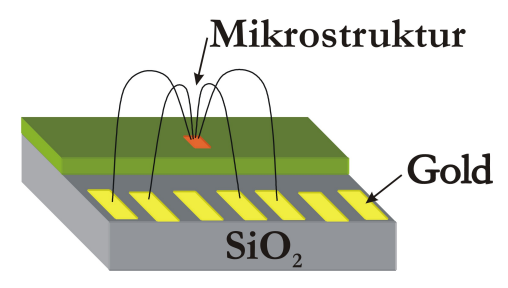

Abbildung 5.15. Schematische Darstellung der Probenkontakierung mittels Bonder.

\subsection{Transportmessungen}

Von wesentlichem Interesse ist das Widerstandsverhalten der Proben in Abhängigkeit des äußeren Magnetfeldes, der Temperatur und der Stromstärke. Hierzu werden zwei Messaufbauten verwendet, deren Spezifikationen im Wesentlichen gleich sind. Es handelt sich einerseits um ein MagLab System ${ }^{2000}$ der Firma Oxford Instruments sowie um ein Physical Properties Measurement System (PPMS) des Herstellers Quantum Design. Beide Systeme machen einen Temperaturbereich von 2-400 K zugänglich, wobei ein äußeres Magnetfeld von bis zu $90 \mathrm{kOe}$ (MagLab), bzw. $70 \mathrm{kOe}$ (PPMS) sowohl in Schichtebene als auch senkrecht dazu angelegt werden kann. Die resistiven Eigenschaften werden mit konventionellen Vier-Punkt-Messungen ermittelt, wobei auf eine Modulation des Probenstroms verzichtet wird. Eine wesentliche Einschränkung des PPMS ist die Limitierung der Ausgangsspannung der Stromquelle auf $95 \mathrm{mV}$, was besonders bei hochohmigen Proben problematisch erscheint. Der Probenstrom muss sehr klein gewählt werden, so dass die Messergebnisse teilweise stark verrauscht sind. Weiterhin ist der zugängliche Bereich einer Strom-Spannungs-Kennlinie deutlich reduziert, was sich insbesondere auf die Bestimmung des Transportprozesses sowie auf das strominduzierte Magnetisierungsschalten negativ auswirkt. Aus diesem Grunde wird das PPMS durch einen temporären Aufbau erweitert, welcher die Temperatur- und Magnetfeldkontrolle des PPMS nutzt, die StromSpannung-Kennlinie jedoch über eine externe Stromquelle (Keithley 2400 SourceMeter) sowie ein externes Spannungsmessgerät (Keithley 2000 Multimeter) computergestützt bestimmt. Hier beträgt die maximale Ausgangsspannung der Stromquelle $20 \mathrm{~V}$, so dass auch bei hochohmigen Proben Stromstärken bis zu einigen Milli-Ampère erreicht werden.

Beide Geräte bieten die Möglichkeit, weitere physikalische Eigenschaften wie Wärmeleitfähigkeit oder $a c$-Suszeptibilität zu bestimmen, worauf im Folgenden aber nicht eingegangen wird. Für weitere Informationen über die Spezifikationen der Geräte sei auf die Literatur verwiesen 184, 185.

Aufgrund der Mikrostrukturierung der Proben stellt die Kontaktierung eine weitere Herausforderung dar. Die Größe der Kontaktflächen liegt im Bereich von ca. $200 \mu \mathrm{m}$ $\times 150 \mu \mathrm{m}$, was eine konventionelle Kontaktierung mittels Leitsilber unmöglich macht. Aus diesem Grunde werden die Kontakte unter Zuhilfenahme eines so genannten Bonders hergestellt. In diesem Falle kommt das Model 4532 der Firma Kulicke \& Soffa Ltd. zum Einsatz. Zur besseren Haftung der Kontakte wird eine Probentemperatur von $50^{\circ} \mathrm{C}$ verwendet. Damit die gleiche Probe in verschiedenen Systemen ohne Entfernen der Bonddrähte gemessen werden kann, wird zusätzlich ein $\mathrm{SiO}_{2}$-Substrat so mit Gold bedampft, 
dass es die einzelnen Kontakte aufnehmen kann und so die Möglichkeit zur weiteren Kontaktierung bietet. Abbildung 5.15 illustriert dieses Vorgehen.

\section{$5.81 / f$-Widerstandsrauschen}

Rauschmessungen erfordern aufgrund ihrer Komplexität und der Anfälligkeiten gegenüber äußeren Störungen besondere Vorkehrungen. Alle Rauschmessungen werden am WaltherMeissner-Institut der Bayerischen Akademie der Wissenschaften in Garching durchgeführt. Der dort verwendete Aufbau wurde speziell für die Messungen von Widerstandsrauschen konzipiert und optimiert. Eine ausführliche Beschreibung des experimentellen Aufbaus ist in [186, 187] gegeben und soll hier kurz erläutert werden.

Da der genutzte Aufbau ursprünglich für die Charakterisierung von HochtemperaturSupraleiter entwickelt wurde und auch für Messungen an hochempfindlichen Magnetometern und Gradiometern eingesetzt werden sollte, wurde besonders auf die Temperaturstabilität im Tieftemperaturbereich $(\mathrm{T} \leq 100 \mathrm{~K})$ sowie auf die Vermeidung magnetischer Störfelder durch einerseits magnetische Materialien oder andererseits fließende Ströme Wert gelegt. Der schematische Aufbau des Kryostaten ist in Abbildung 5.16 dargestellt. An der Grundplatte eines evakuierbaren Probenraums ist ein GFK ${ }^{(10)}$ Tragrohr befestigt, welches einerseits zur Aufnahme der Probe und andererseits zur schwachen Ankopplung an das Kältebad dient. Da die Richtung des Magnetfeldes, welches durch den im Heliumbad befindlichen, supraleitenden Magneten erzeugt wird, senkrecht zur eigentlichen Probenaufnahme und somit bei der Charakterisierung dünner Schichten senkrecht zu dieser verläuft, wird die eigentliche Probenbefestigung dahingehend erweitert, dass mittels eines thermisch gut angekoppelten $\mathrm{Cu}$-Winkels die Schichtebene parallel zum externen Magnetfeld verläuft (siehe Abbildung 5.17). Die eigentliche Probenhalterung besteht dabei aus einem einseitig polierten Si-Wafer mit einem Durchmesser von einem Zoll und einer Dicke von einem Millimeter. Die Grundplatte des Probenraums ist mit einem dünnwandigen Neusilberrohr verlötet, welches alle Versorgungsleitungen aufnimmt und über welches die Evakuierung des Probenraums erfolgt. Hierüber kann zur besseren thermischen Ankopplung gegebenfalls auch Austauschgas eingelassen werden.

Die Versorgungsleitungen werden paarweise verdrillt zur zusätzlichen Abschirmung in zwei Neusilberrohren geführt, wobei Leitungen mit Kontakt zur Probe getrennt von den Versorgungsleitungen des Temperatursensors geführt werden. Durch eine Vakuumdurchführung auf koaxiale Anschlüsse ist eine weitere Kontaktierung gewährleistet.

Ein besonderes Augenmerk ist der Umsetzung der Heizung zu widmen. Aufgrund von elektromagnetischen Störungen, welche resistive Widerstandsheizungen auf hochempfindliche Rauschmessungen haben können, erfolgt die Temperaturkontrolle des Si-Wafers samt Probe über eine optische Heizung. Eine Laserdiode erzeugt außerhalb der magnetischen Abschirmkammer (siehe unten) eine Heizleistung, welche in einen Lichtwellenleiter, der ebenfalls durch das Neusilbertragrohr verläuft, eingekoppelt wird. Das Ende der Glasfaser wird im Probenraum in einer Kanüle verklebt und in einem Abstand von ca. $50 \mathrm{~mm}$ von

${ }^{(10)}$ Glasfaser verstärkter Kunststoff 


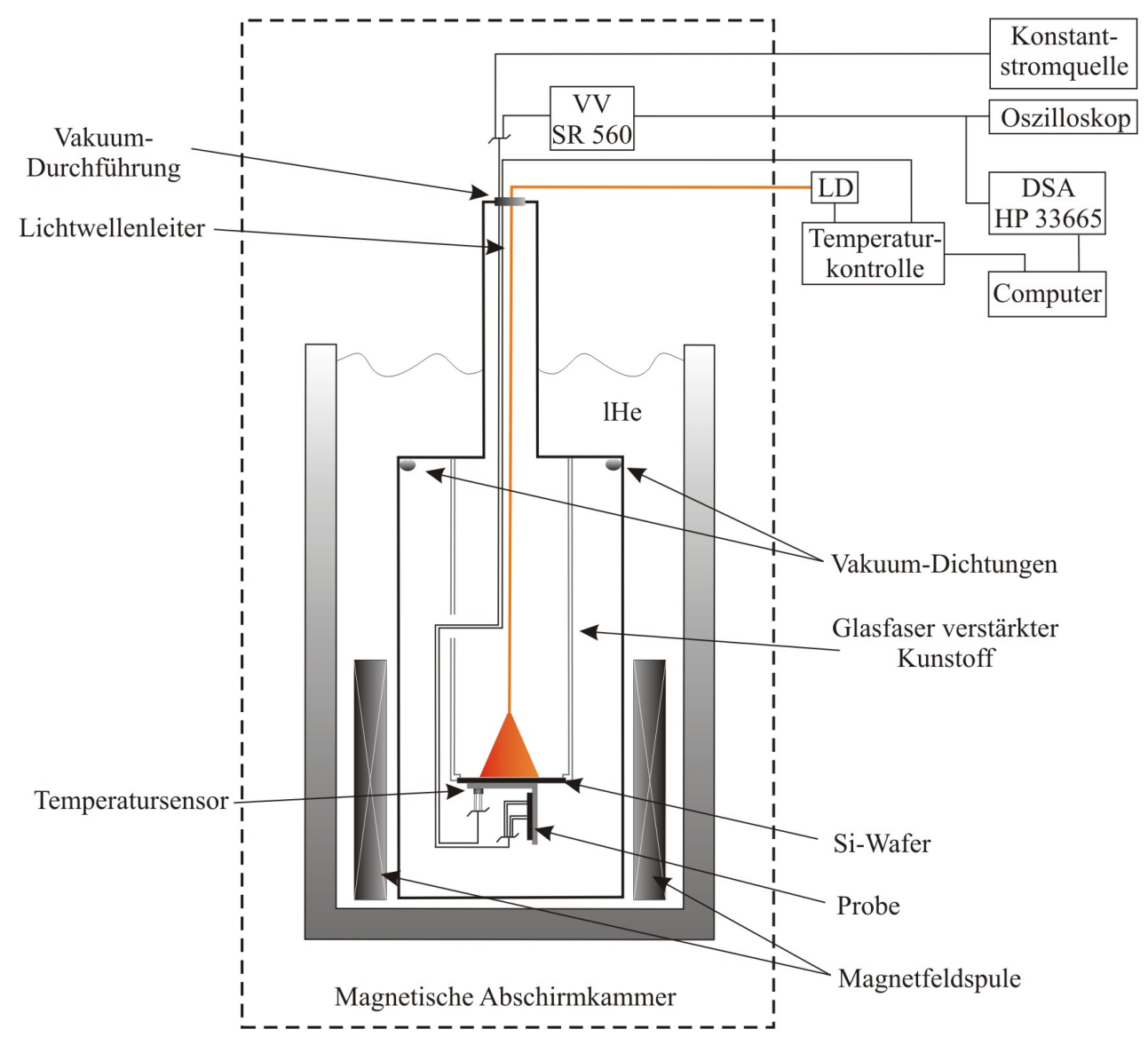

Abbildung 5.16. Schematischer Aufbau des Kryostaten zur Durchführung der Rauschmessungen. Nach [187].

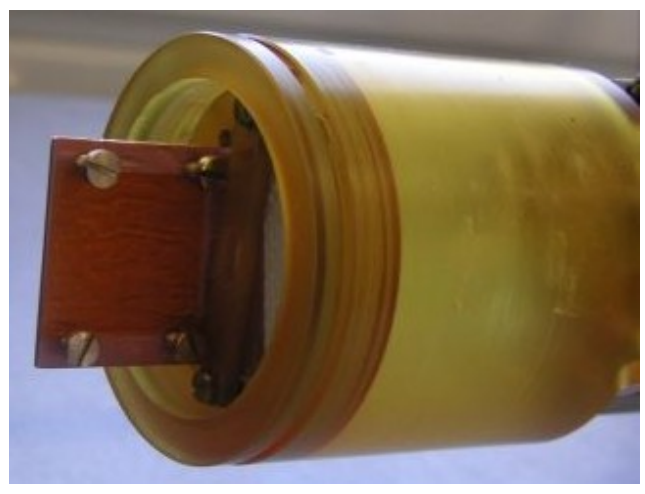

Abbildung 5.17. Modifizierung des Probenhalters zur Durchführung von Rauschmessungen mit parallel zur Schichtebene verlaufendem Magnetfeld. 


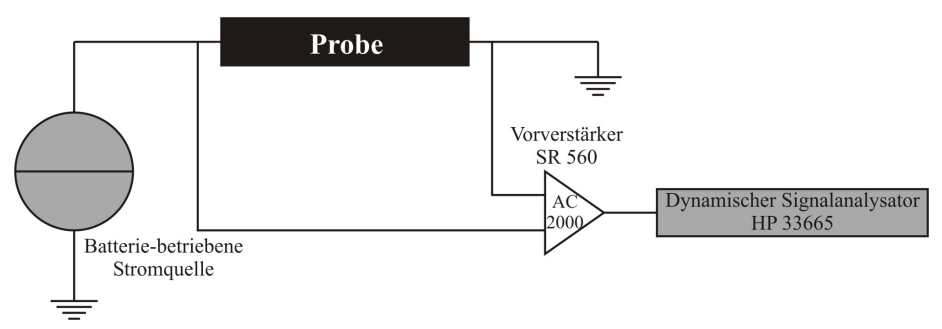

Abbildung 5.18. Schaltskizze zur Rauschanalyse in 4-Punkt-Geometrie.

der Rückseite des Si-Wafers fixiert, welche zur Reduktion von Reflexionen angeätzt ist. Die Temperaturmessung erfolgt über eine Si-Diode, welche sich möglichst nah und in gutem thermischen Kontakt zur Probe befindet. Zur Temperatursteuerung wird ein LTC21 der Firma Neocera verwendet. Aufgrund der begrenzten Heizleistung der Diode ist die maximal zugängliche Temperatur auf $100 \mathrm{~K}$ limitiert.

Die zur Rauschmessung verwendete Schaltung ist in Abbildung 5.18 dargestellt. Zur Vermeidung der Einkopplung eventueller Netzfrequenzen wird der Aufbau soweit wie möglich vom Stromnetz abgekoppelt. Daher wird die Stromquelle, welche eine Anfertigung des Walther-Meissner-Instituts ist, batteriebetrieben und ermöglicht so das Einspeisen eines sehr rauscharmen Stromes. Die an der Probe abfallende Spannung wird in einen ebenfalls batteriebetrieben Vorverstärker SR560 der Firma Stanford Research im ac-Modus eingekoppelt und um den Faktor 2000 verstärkt. Das Ausgangssignal des Vorverstärkers wird schließlich in den digitalen Spektrums-Analysator HP33665, hergestellt von HewlettPackard, geleitet und mittels eines Computerprogramms [188 aufgenommen. Dabei wird vor jeder Messung bei konstanter Stromstärke zunächst der Untergrund gemessen, d.h. es wird das Spannungsrauschen für $I=0$ gemessen. Da zur Bestimmung des Widerstandsrauschens der Probe eine endliche Stromstärke benötigt wird, stellt dieses Rauschen den Untergrund dar. Das erhaltene Spektrum wird von jeder Messung, welche bei sonst gleichen Bedingungen durchgeführt wird (also identisches externes Magnetfeld und identische Temperatur), abgezogen.

Alle Messungen werden zur weiteren Erhöhung der Sensitivität in einer magnetischen Abschirmkammer durchgeführt. Diese dient auch gleichzeitig als definierter Massepunkt aller verwendeten Geräte. Dabei wird der batteriebetriebene Vorverstärker innerhalb der Kammer und möglichst nah am Kryostaten positioniert, um den Einfluss auf das unverstärkte Signal möglichst zu minimieren. Alle weiteren Geräte sind außerhalb der Kammer angebracht und werden über Koaxialverbindungen in die Kammer eingekoppelt. Um weitere Störungen von außen zu vermeiden, werden viele Messungen am späten Abend und am Wochenende durchgeführt. 



\section{Kapitel 6}

\section{Nanokomposite}

Aufgrund ihrer faszinierenden physikalischen Eigenschaften haben Nanokomposite großes Interesse geweckt, nicht zuletzt infolge ihrer sehr viel einfacheren Präparation im Gegensatz zu Dünnschicht-Heterostrukturen. In diesem Kapitel werden die Ergebnisse der in dieser Arbeit untersuchten Nanokomposite LSMO:MgO (Kap. 6.1), LSMO:CeO ${ }_{2}$ (Kap. 6.2) und des reinen, nanokristallinem LSMO (Kap. 6.3) vorgestellt und anschließend diskutiert. Dabei ist der Schwerpunkt jeweils unterschiedlich gelagert: Im System LSMO:MgO liegt er auf der Charakterisierung der Grenzflächen mittels niederfrequenter 1/f-Rauschspektroskopie, für LSMO: $\mathrm{CeO}_{2}$ ist der Einfluss der Anzahl der in den Ladungstransport involvierten Körner von besonderem Interesse, während für das reine System strom- bzw. spannungsinduzierte Effekte vorgestellt werden.

\subsection{LSMO:MgO Nanokomposite}

In den Arbeiten von Köster et al. 29, 76 konnte gezeigt werden, dass im System LSMO:MgO eine chemischen Phasenseparation zwischen dem ferromagnetischen, metallischen LSMO und dem isolierenden $\mathrm{MgO}$ auftritt. Unter Verwendung von $\mathrm{Al}_{2} \mathrm{O}_{3^{-}}$ Substraten kommt es zur Ausbildung eines Nanokomposits, worin die einzelnen LSMOKörner durch MgO getrennt sind, so dass auf natürliche Weise ein laterales TMR-System gebildet wird (s. Kap. 2.5.2). Der Einfluss der Grenzflächen LSMO:MgO auf diesen Kleinfeld-Magnetowiderstand ist aber noch immer ungeklärt, insbesondere ist die Frage nach einem gestörten Bereich des Manganats offen. Ein geeignetes Mittel zur Analyse dieser Fragestellung ist die hier angewandte niederfrequente $1 / f$-Rauschanalyse.

LSMO:MgO Nanokomposite mit einem MgO-Gehalt von $5 \%$ bis zu $33 \%$ werden mittels MAD-Technik präpariert. Die Dicke der auf $\mathrm{Al}_{2} \mathrm{O}_{3}$ deponierten Filme beträgt ca. $50 \mathrm{~nm}$, was über das Lösungsvolumens bei der Präparation eingestellt und anschließend durch Röntgenreflektometrie kontrolliert wird. Aufgrund des zunehmenden elektrischen Widerstandes der Proben mit dem MgO-Gehalt und ein in der Leistung limitiertes Netzteil kann nur die Probe mit 5\% MgO durch die Rauschspektroskopie charakterisiert werden. Im Weiteren wird deshalb nur diese Probe vorgestellt. 

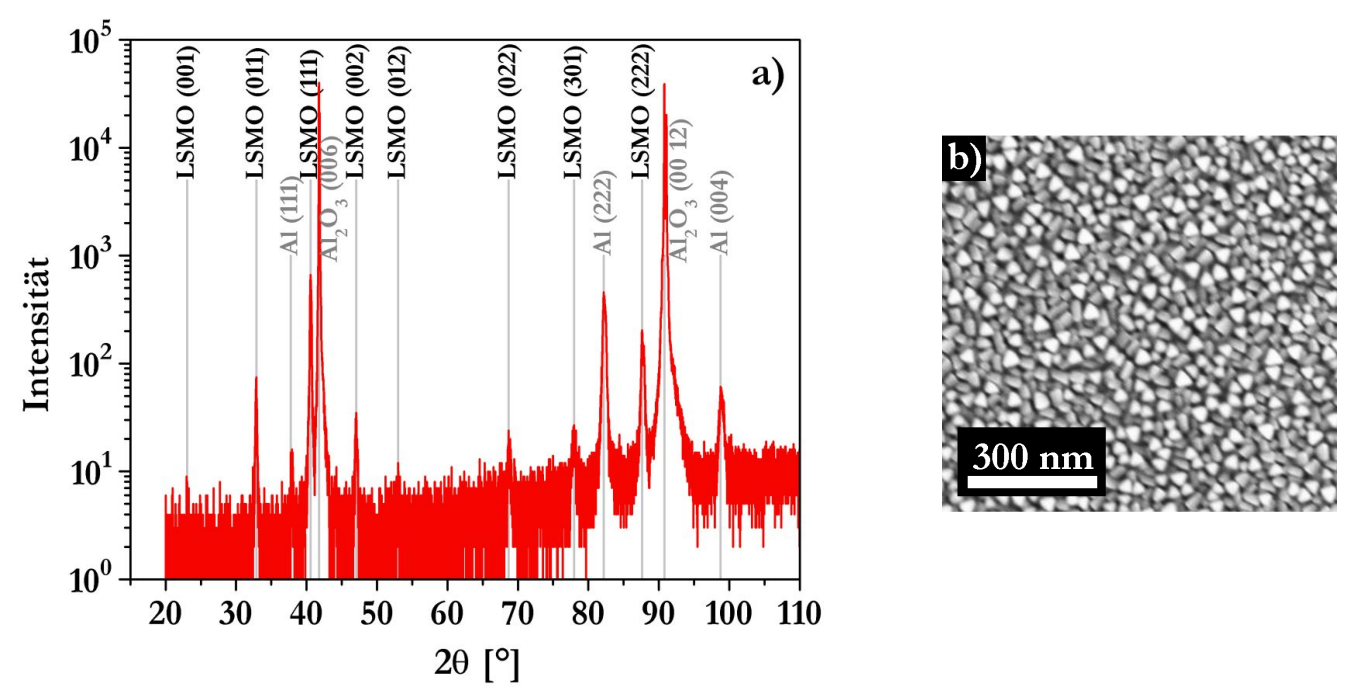

Abbildung 6.1. a) Röntgenstrukturanalyse einer LSMO:MgO Nanokompositschicht mit einem MgO-Anteil von 5\%. b) REM-Aufnahme der Oberfläche.

\subsubsection{Strukturanalyse}

Die strukturellen Eigenschaften der hergestellten Nanokomposite werden mittels Röntgendiffraktometrie [Abb. 6.1 a)] und REM-Aufnahmen [Abb. 6.1 b)] charakterisiert. Da die Probe den Aluminium-Probenhalter des Röntgendiffraktometers nicht vollständig bedeckt, erscheinen auch Aluminium-Reflexe im Röntgendiffraktogramm, welche nicht weiter diskutiert werden. Alle weiteren Reflexe können dem Substrat und LSMO zugeordnet werden, wobei für LSMO Reflexe verschiedener Orientierung auftreten. Neben den dominanten Substratreflexen haben die LSMO-Reflexe vom Typ $(h h h)$ die höchste Intensität, das Wachstum ist also polykristallin mit einer vorherrschenden (111)-Orientierung. Die Größe $\tau$ der in $q$-Richtung kohärent streuenden Bereiche kann durch die ScherrerFormel [175] über die Halbwertsbreite der Reflexe berechnet werden. Eine Analyse des LSMO-(111)-Reflexes führt zu $\tau=44 \mathrm{~nm}$. Über die Lage aller LSMO-Reflexe kann der Gitterparameter von LSMO berechnet werden, welcher sich zu $a=3,86 \AA$ ergibt. Weiterhin zeigen sich keine Reflexe, welche MgO zugeordnet werden können.

Die REM-Aufnahme der Oberfläche [Abb. 6.1 b)] ermöglicht die Bestimmung der lateralen Korngröße, welche mit der Röntgenanalyse nicht zugänglich ist. Deutlich sind zumeist dreieckige Strukturen zu erkennen, welche wiederum auf eine dominante (111)Wachstumsorientierung deuten. Die mittlere Größe dieser Strukturen beträgt ca. $40 \mathrm{~nm}$.

\subsubsection{Magnetotransport}

Zur Bestimmung der resistiven Eigenschaften wird die Probe mit optischen Methoden am Walther-Meissner-Institut, Garching strukturiert. Die Messungen werden an einem $300 \mu \mathrm{m}$ langen und $30 \mu \mathrm{m}$ breiten Streifen durchgeführt.

Abbildung 6.2 zeigt das resistive Verhalten im Vergleich mit der Magnetisierung als Funktion der Temperatur (a), bzw. als Funktion des äußeren Feldes (b). Die Temperatur- 

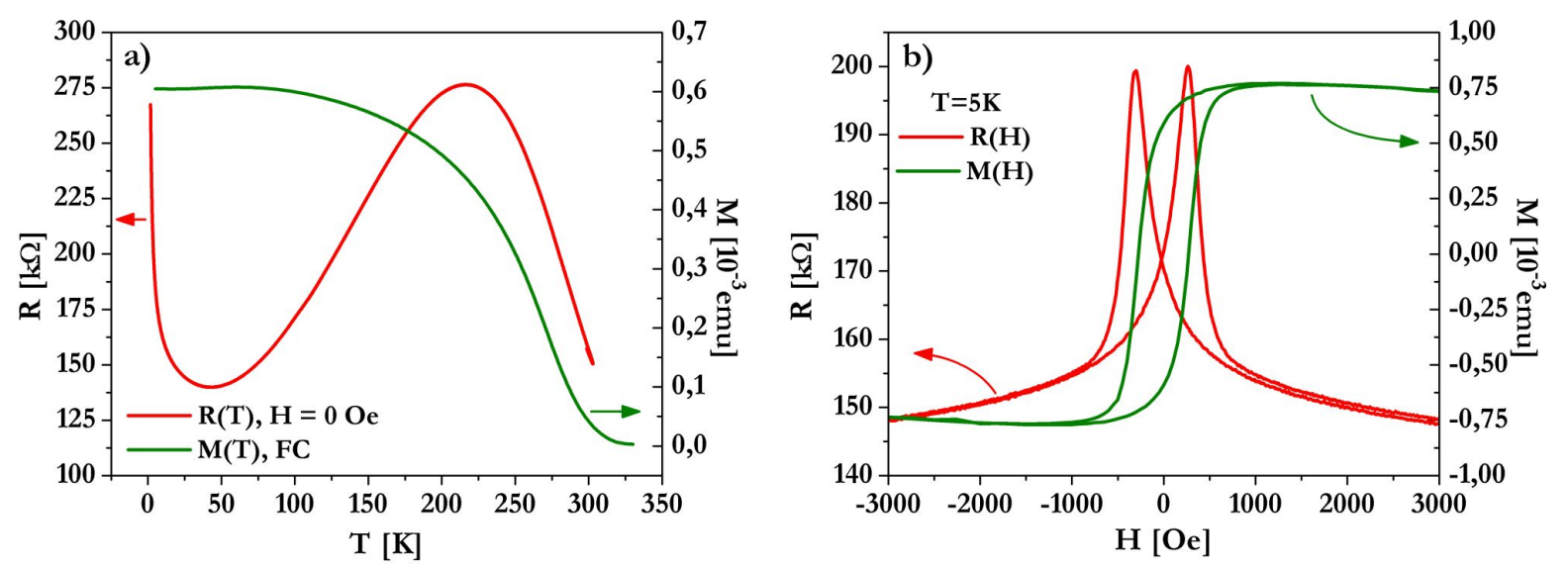

Abbildung 6.2. a) Temperaturabhängigkeit der Magnetisierung ( $H=100 \mathrm{Oe})$ und des elektrischen Widerstandes $(I=1 \mu \mathrm{A})$. b) Magnetfeldabhängigkeit der Magnetisierung und des elektrischen Widerstandes bei $T=5 \mathrm{~K}(I=1 \mu \mathrm{A})$.

abhängigkeit der Magnetisierung, welche in einem äußeren Feld von 100 Oe gemessen wird, weist ein ferromagnetisches Verhalten unterhalb der Curie-Temperatur $T_{C} \approx 315 \mathrm{~K}$ auf, welche durch das Minimum in $(1 / M)(\partial M / \partial T)$ bestimmt wird. Die Temperaturabhängigkeit des elektrischen Widerstandes zeigt für $T \leq 35 \mathrm{~K}$ einen starken Anstieg zu tiefen Temperaturen. Im Temperaturbereich $35 \mathrm{~K} \leq T \leq 220 \mathrm{~K}$ ergibt sich ein mit zunehmender Temperatur ansteigender Widerstand. Für $T \geq 220 \mathrm{~K}$ hingegen zeigt der Widerstand ein isolierendes Temperaturverhalten, s.d. zwischen der Temperatur maximalen Widerstandes und der Curie-Temperatur eine Temperaturdifferenz von ca. $95 \mathrm{~K}$ liegt.

Die Magnetfeldabhängigkeit der Magnetisierung sowie des Widerstandes bei $T=5 \mathrm{~K}$ ist in Abbildung 6.2 b) dargestellt. Die Magnetisierung zeigt eine typische, ferromagnetische Hysterese mit einem Sättigungsfeld $H_{S}$ von ca. 950 Oe und einem Koerzitivfeld $H_{C}$ von ca. 275 Oe. Der Widerstand zeigt eine ausgeprägte Abhängigkeit vom äußeren Feld und ebenfalls eine Hysterese in kleinen Feldern $\left(H \leq H_{S}\right)$. Durch den bei $H=H_{C}$ maximalen Widerstand ergibt sich ein weiterer Zusammenhang mit der Hysterese der Magnetisierung. Für höhere Felder ist eine nichthysteretische Abnahme des Widerstandes zu beobachten, welche keine Anzeichen einer Sättigung aufweist.

Zur quantitativen Beschreibung der großen Widerstandsänderung in kleinen Feldern (engl.: low-field magnetoresistance, LFMR) wird folgende Definition herangezogen:

$$
M R=\frac{R(H)-R(1000 \mathrm{Oe})}{R(1000 \mathrm{Oe})}=\frac{\Delta R}{R}
$$

Die Normierung soll so gewählt werden, dass der Einfluss der nichthysteretischen Widerstandsänderung möglichst gering ist, der LFMR aber dennoch voll erfasst wird. Da das Sättigungsfeld bei 950 Oe liegt, erscheint die Normierung auf den Widerstandswert von 1000 Oe sinnvoll. Mit dieser Definition ergibt sich ein Magnetowiderstandseffekt von fast $30 \%$ für $T=5 \mathrm{~K}$.

Der Magnetowiderstand, gemessen bei verschiedenen Temperaturen, ist in Abbildung 6.3 a) dargestellt. Die Felder maximalen MRs nehmen mit steigender Temperatur aufgrund 

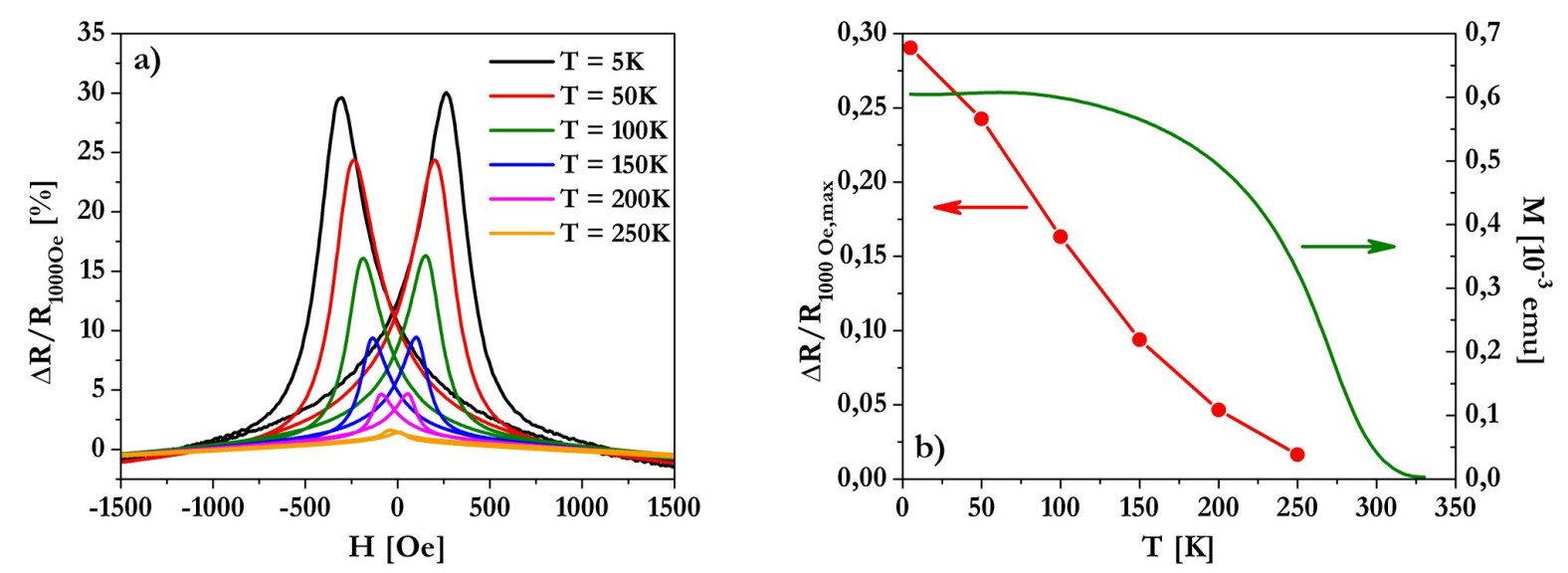

Abbildung 6.3. a) Magnetfeldabhängigkeit des elektrischen Widerstandes für verschiedene Temperaturen ( $I=1 \mu \mathrm{A})$. b) Temperaturabhängigkeit des Kleinfeld-Magnetowiderstandes im Vergleich mit der Magnetisierung.

der thermisch aktivierten Umschaltprozesse der Magnetisierung ab. Weiterhin zeigt sich, dass der Maximalwert des Magnetowiderstandes systematisch mit zunehmender Temperatur abnimmt. Für $T=200 \mathrm{~K}$ ist noch ein messbarer MR vorhanden, während das Verhalten für $T=250 \mathrm{~K}$ keinen reproduzierbaren MR aufweist, da die Widerstandshysterese asymmetrisch ist. Die Maximalwerte des Magnetowiderstandes als Funktion der Temperatur werden in Abbildung 6.3 b) mit der Temperaturabhängigkeit der Magnetisierung verglichen. Der MR weist ein auffallend anderes Temperaturverhalten als die Magnetisierung auf und verschwindet bei deutlich tieferen Temperaturen als die Magnetisierung.

\subsection{3 $1 / f$-Widerstandsrauschen}

Um die Grenzflächeneigenschaften der Nanokomposite genauer zu charakterisieren, werden systematische $1 / f$-Rauschanalysen an der Nanokomposit-Probe durchgeführt. Wie in Abschnitt 5.8 beschrieben, ist hierbei lediglich der Temperaturbereich $T \leq 100 \mathrm{~K}$ zugänglich, d.h. das Intervall, in dem der Widerstand ein Maximum aufweist und der Magnetowiderstand auf Null reduziert wird, ist nicht zugänglich. Die spektrale Rauschleistungsdichte $S_{U}$ wird in Abhängigkeit der Temperatur und des äußeren Magnetfeldes für verschiedene Stromstärken $I$ ermittelt, wobei die Spektren durch Abzug der Messung mit $I=0$ untergrundbereinigt sind. Die maximale Stromstärke beträgt probenbedingt $7 \mu \mathrm{A}$, das maximale äußere Magnetfeld 1024 Oe. Somit ist gewährleistet, dass die Probe bei maximalem Feld gesättigt ist [s. Abb. 6.2 b)].

Abbildung 6.4 zeigt exemplarisch die ermittelte Rauschleistungsdichte bei $T=25 \mathrm{~K}$ und $H=0$ Oe für unterschiedliche Stromstärken. Die einzelnen Spektren sind dabei das Ergebnis einer Mittelung über jeweils 100 Messdurchläufe.

Alle aufgenommenen Spektren zeigen unterhalb von $1000 \mathrm{~Hz}$ ein reines $1 / f^{\alpha}$-Verhalten mit $0,8 \leq \alpha \leq 1,2$. Ein zusätzlicher Anteil wie etwa ein Lorentz-Maximum, welches auf ein Zwei-Niveau-System deuten würde (siehe Kapitel 4.4), ist für keines der gewonnenen Spektren zu beobachten. Weiterhin wird in Abbildung 6.4 deutlich, dass $S_{U}$ für höhere Stromstärken ansteigt. 


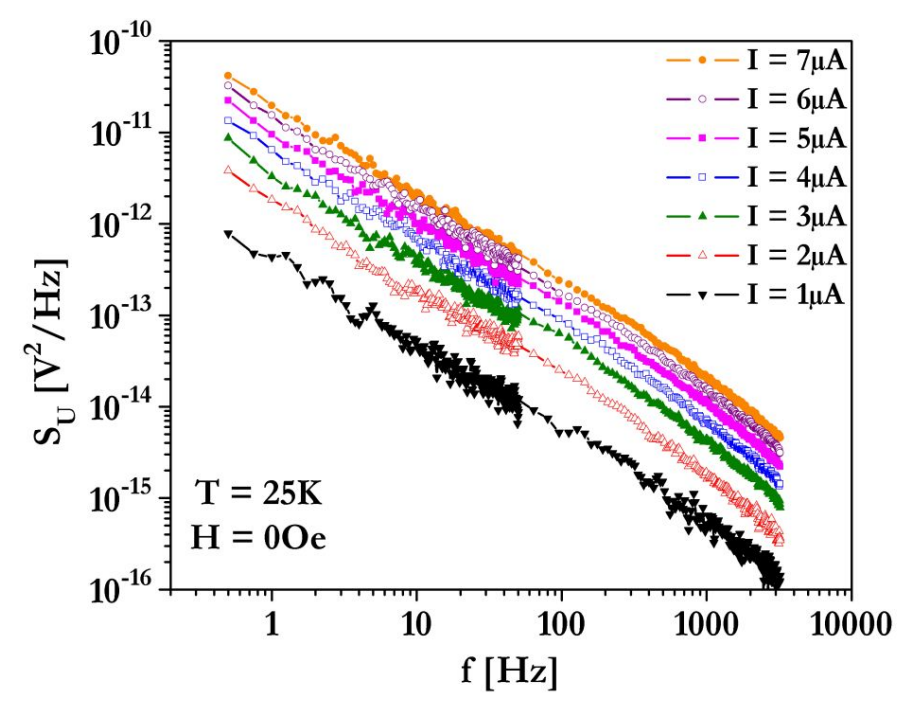

Abbildung 6.4. Typische spektrale Spannungsrauschleistungsdichte für $T=25 \mathrm{~K}$ und $H=0$ Oe, gemessen für verschiedene Stromstärken.

Um neben den intrinsischen Widerstandsfluktuationen weitere Rauschursachen auszuschließen (Kontaktrauschen, eingekoppelte Störungen von außerhalb o.ä.), wird für jede Temperatur die Stromabhängigkeit der gewonnenen Spektren analysiert. Da als Messgröße die spektrale Spannungsrauschleistungsdichte dient, sollte sich eine quadratische Abhängigkeit vom angelegten Strom zeigen. Hierzu wird der Parameter $\Gamma$ definiert:

$$
\Gamma:=\int_{10 \mathrm{~Hz}}^{20 \mathrm{~Hz}} S_{U} d f
$$

Die spektrale Rauschleistungsdichte ist bei konstanter Stromstärke proportional zum Quadrat der Abweichung $\delta U$ der abgegriffenen Spannung vom Mittelwert $\bar{U}$, woraus sich aus dem Ohmschen Gesetz eine Abhängigkeit von $I^{2}$ äußert [145]:

$$
S_{U} \propto(\delta U)^{2}=I^{2}(\delta R)^{2}
$$

Ergibt sich also bei einer Auftragung von $\Gamma$ gegen das Quadrat der Stromstärke eine Nullpunktsgerade, so können weitere Rauschquellen als Ursache für das gemessene Signal ausgeschlossen und die intrinsischen Widerstandsfluktuationen als dominant angenommen werden. Abbildung 6.5 zeigt einen repräsentativen Verlauf von $\Gamma$ in Abhängigkeit von $I^{2}$ bei $T=25 \mathrm{~K}$ und $H=0 \mathrm{Oe}$, wobei dieses Verhalten für alle verwendeten Temperaturen und Magnetfelder gefunden wird. Es zeigt sich deutlich ein linearer Zusammenhang zwischen $\Gamma$ und $I^{2}$, s.d. das Rauschen im Wesentlichen nicht durch äußere, nicht stromgetriebene Prozesse verursacht sein kann. Auch die Stromquelle kann nicht für das Rauschen verantwortlich sein, da $\Gamma$ bei gleichbleibender Stromstärke mit dem Widerstand skaliert, welcher seinerseits über den Magnetowiderstandseffekt durch das äußere Magnetfeld variiert werden kann. Somit resultiert das gemessene Rauschen aus intrinsischen 


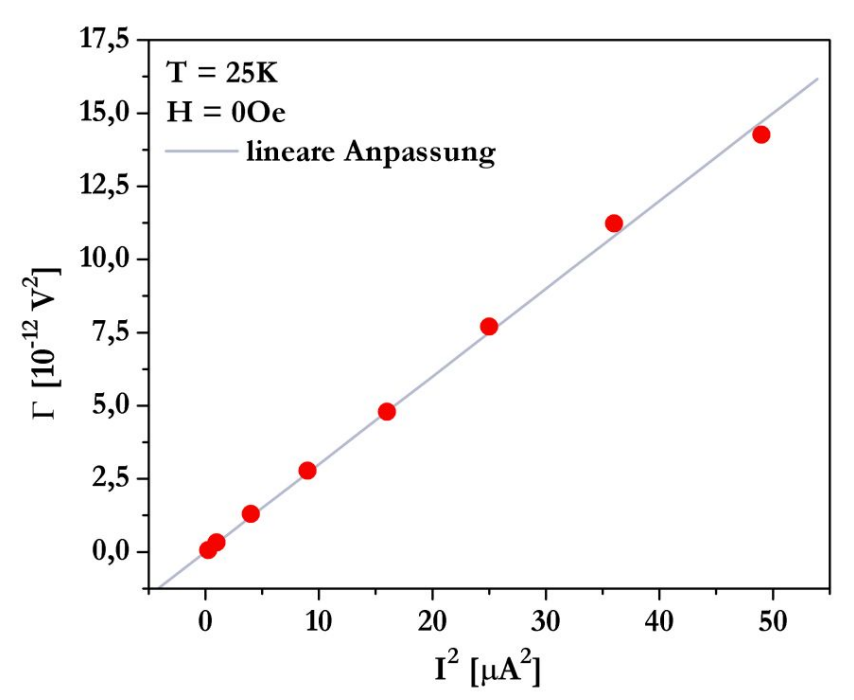

Abbildung 6.5. Abhängigkeit des Parameters $\Gamma$ vom angelegten Strom für $T=25 \mathrm{~K}$ und $H=0$ Oe. Bei Auftragung gegen $I^{2}$ ergibt sich eine Nullpunktsgerade.

Widerstandsfluktuationen.

Um die intrinsischen Widerstandsfluktuationen auch quantitativ analysieren zu können, sollte der Hooge-Parameter $\gamma$ herangezogen werden (s. Kap. 4.4.2). Hierzu wird die Ladungsträgerkonzentration $n$ benötigt, welche allerdings für solch komplexe Strukturen, wie das hier vorliegende Nanokomposit, a priori nicht zugänglich ist. Daher wird der Parameter $a_{U}$ definiert, welcher ebenfalls eine quantitative Analyse erlaubt, aber unabhängig von der Ladungsträgerkonzentration ist 154:

$$
a_{U}:=\frac{\gamma}{n}=S_{U} \frac{\Omega \cdot f^{\alpha}}{U^{2}}
$$

Aufgrund der Multiplikation von $S_{U}$ mit $f^{\alpha}$, wobei der Exponent $\alpha$ aus einer Anpassung einer zu $1 / f^{\alpha}$-proportionalen Funktion an die gemessene Rauschleistungsdichte $S_{U}$ gewonnen wird, ist der Parameter $a_{U}$ frequenzunabhängig. Durch die Normierung auf das Probenvolumen $\Omega$ und das Quadrat der abgefallenen Spannung $U$ wird ein Vergleich mit anderen Proben möglich. Zur weiteren Analyse wird $a_{U}$ im gesamten Bereich $10 \mathrm{~Hz} \leq f \leq$ $20 \mathrm{~Hz}$ berechnet und anschließend über alle Werte gemittelt. Der in den Auftragungen dargestellte Fehler ist der hierbei entstehende Mittelwertfehler. Weiterhin sollte beachtet werden, dass der Parameter $a_{U}$ proportional zu Widerstandsfluktuationen $(\delta R / R)^{2}$ ist [145], was für die spätere Interpretation der Daten relevant ist.

In Abbildung 6.6 a) ist die Magnetfeldabhängigkeit des Parameters $\left\langle a_{U}\right\rangle$ für $T=100 \mathrm{~K}$ und $I=5 \mu \mathrm{A}$ dargestellt, wobei der Verlauf für alle analysierten Temperaturen und Stromstärken repräsentativ ist. Das normierte Rauschen zeigt innerhalb der Fehlergrenzen keine Abhängigkeit vom äußeren Magnetfeld.

Im Gegensatz zum äußeren Magnetfeld hat die Temperatur einen Einfluss auf die normierte Rauschamplitude. Abbildung 6.6 b) zeigt das temperaturabhängige Verhalten von $\left\langle a_{U}\right\rangle$ für $H=1026$ Oe und $I=5 \mu \mathrm{A}$, wobei ein deutliches Maximum um $T=25 \mathrm{~K}$ 

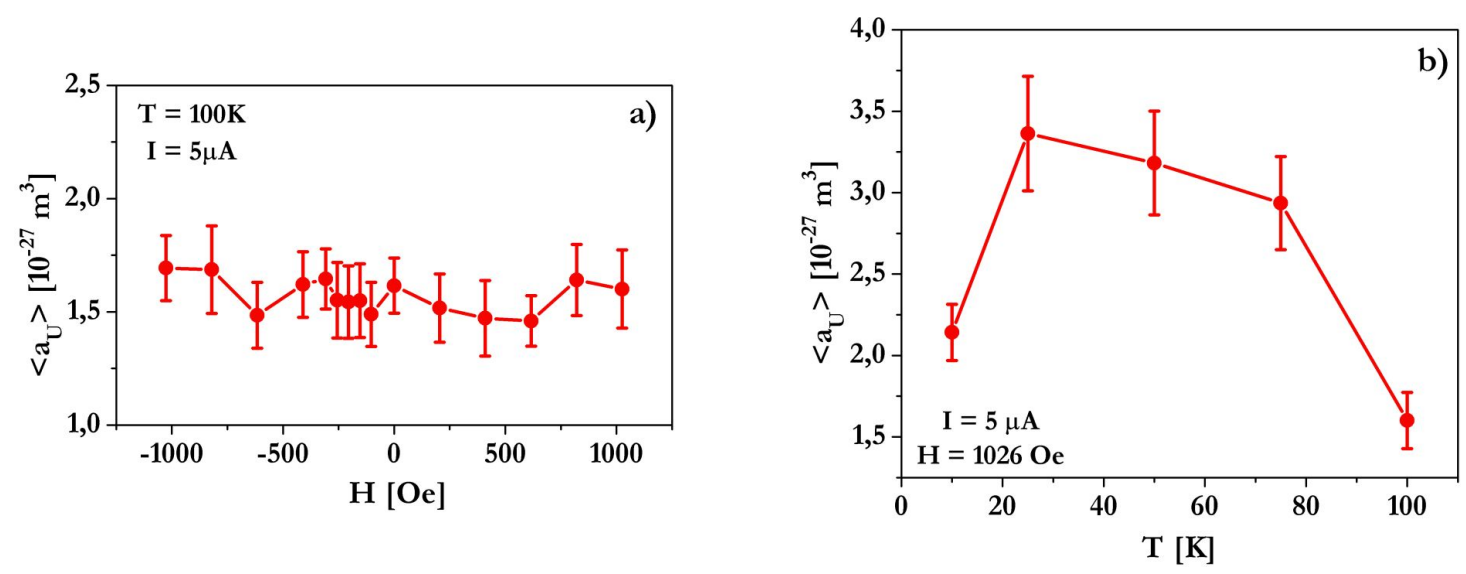

Abbildung 6.6. a) Magnetfeldabhängigkeit des Parameters $\left\langle a_{U}\right\rangle$ für $T=100 \mathrm{~K}$ und $I=$ $5 \mu \mathrm{A}$. b) Temperaturabhängigkeit des Parameters $\left\langle a_{U}\right\rangle$ für $H=1026$ Oe und $I=5 \mu \mathrm{A}$.

zu erkennen ist, also in dem Temperaturbereich, in dem die Temperaturabhängigkeit des Widerstandes ein Minimum aufweist (s. Abb. 6.2). Dieses temperaturabhängige Verhalten ist wiederum repräsentativ für alle untersuchten Magnetfelder und Stromstärken.

\subsubsection{Diskussion}

\section{Struktur}

Die Ergebnisse der strukturellen Charakterisierung deuten auf kolumnares Wachstum von LSMO-Säulen hin, wie es bereits von Köster et al. [29,76 durch TEM-Untersuchungen nachgewiesen werden konnte. Das Röntgendiffraktogramm zeigt zunächst den polykristallinen Charakter der Schicht, da mehrere Kristallorientierungen parallel zur Schichtnormalen auftreten [Abb. 6.1 a)]. Aus der Intensitätsverteilung kann geschlossen werden, dass eine dominante (111)-Orientierung der Körner vorliegt. Ebenfalls kann mittels der Scherrer-Formel die Größe der in Richtung der Schichtnormalen kohärent streuenden Bereiche berechnet werden, welche sich für den (111)-Reflex zu $44 \mathrm{~nm}$ ergibt. Diese liegt somit im Bereich der Schichtdicke, s.d. angenommen werden kann, dass die (111)-orientierten Körner jeweils säulenartig vom Substrat bis zur Oberfläche reichen. Der geringfügige Unterschied zwischen der Größe der kohärent streuenden Bereiche und der Schichtdicke kann neben Oberflächenrauigkeiten dadurch erklärt werden, dass ein Korn nicht vollständig kohärent streut. Es kann also auf kolumnares Wachstum geschlossen werden.

Ein direkter Nachweis dafür, dass MgO einzelne LSMO-Säulen trennt, kann mittels Röntgendiffraktometrie nicht gelingen. Dennoch weisen alle Ergebnisse darauf hin, dass hier ein identischer Fall vorliegt wie bei Köster et al. |76|. Deshalb wird im Weiteren davon ausgegangen, dass einzelne LSMO-Körner mit einem mittleren Durchmesser von ca. $40 \mathrm{~nm}$ durch eine dünne $\mathrm{MgO}$-Barriere getrennt sind. 


\section{Magnetotransport}

Der beobachtete Magnetowiderstand ist auf zwei Ursachen zurückzuführen: einerseits auf den CMR, der insbesondere für hohe magnetische Felder wichtig ist (s. Kap. 2.2.1) und andererseits auf das spinpolarisierte Tunneln von Leitungselektronen über die isolierenden Barrieren. Der zweite Effekt ist abhängig von der relativen Orientierung der Magnetisierungen benachbarter Körner und somit direkt verknüpft mit der magnetischen Hysterese. Dies wird in Abbildung 6.2 b) deutlich: Sind die magnetischen Momente der Probe statistisch verteilt $\left(M=0, H=H_{C}\right)$, so ist die Tunnelwahrscheinlichkeit gering und der Widerstand am höchsten. Ist die Magnetisierung gesättigt, ist der Widerstand gering.

Der polykristalline Charakter der Probe äußert sich ebenfalls im Tieftemperaturverhalten des elektrischen Widerstandes. Der starke Anstieg zu tiefen Temperaturen hin wird vermutlich durch das Tunneln der Leitungselektronen über die Korngrenzen verursacht. Genauere Analysen des Widerstandsverlaufes (nicht dargestellt) ergeben, dass auch anderere Transportmechanismen wie eine Coulomb-Blockade [189,190] oder das variable range hopping [191 ein Rolle spielen können.

Besonders die Temperaturabhängigkeiten der elektronischen Eigenschaften lassen die Frage nach einem gestörten Bereich des LSMO an der Grenzfläche LSMO/MgO aufkommen. Hierfür spricht einerseits die Tatsache, dass der Magnetowiderstand oberhalb von $200 \mathrm{~K}$, also signifikant unterhalb von $T_{C}$, verschwindet. Weiterhin liegt das Maximum der temperaturabhängigen Widerstandskurve ebenfalls deutlich unterhalb $T_{C}(\Delta T \approx 95 \mathrm{~K})$. Für einkristalline Manganate und epitaktische Schichten wird üblicherweise erwartet, dass die beiden Phasenübergänge nahezu gleichzeitig auftreten (s. Kap. 2.2.4). Auch die Form der Magnetisierungskurve nahe des Phasenübergangs ist nicht so ausgeprägt wie für epitaktische, reine MAD-Manganat Proben. Die Annahme eines strukturell und/oder elektronisch gestörten Bereiches kann diese Phänomene leicht erklären. So ist der Widerstand maßgeblich durch die Korngrenzen beeinflusst, da jedes Leitungselektron diese durchqueren muss. Die Magnetisierung hingegen stellt eine Volumenmessung dar. Hat das Innere eines Korns eine höhere Curie-Temperatur als ein Bereich an der Grenzfläche zum Isolator, so sollte sich dieses im Wesentlichen nur im Widerstand äußern. Die gemessene Curie-Temperatur sollte dem Inneren des Korns entsprechen. Da der LFMR sehr sensitiv auf Grenzflächeneigenschaften reagiert, ist auch der schnelle Abfall mit steigender Temperatur durch diese Hypothese erklärbar. Gegen die hier aufgestellte Hypothese eines gestörten Bereichs an der Grenzfläche spricht der sehr hohe Wert des LFMR, zumindest bei tiefen Temperaturen. Die Widerstandsänderung von 30\% in kleinen Feldern liegt etwas über den Werten, welche zuvor für dieses System gefunden wurden 29,76, und somit auch deutlich über Werten für Systeme mit anderen Isolatoren, siehe z.B. |66.

\section{$1 / f$-Widerstandsrauschen}

Die niederfrequente $1 / f$-Rauschspektroskopie ist besonders sensitiv auf Grenzflächeneigenschaften (s. Kap. 4.4) und bietet somit ein geeignetes Mittel zur Aufklärung der oben aufgestellten Hypothese. Im Rahmen der experimentellen Fehler ist der Parameters $a_{U}$ unabhängig vom äußeren Magnetfeld. Hieraus können zwei wesentliche Schlüsse gezogen 


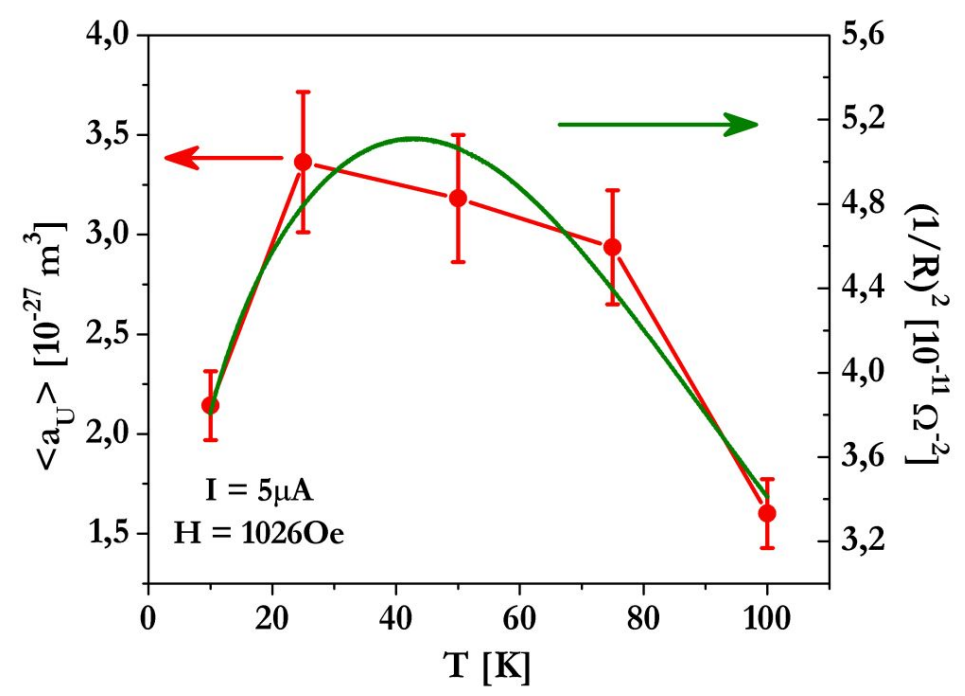

Abbildung 6.7. Temperaturabhängigkeit des Parameters $\left\langle a_{U}\right\rangle$ für $H=1026$ Oe und $I=$ $5 \mu \mathrm{A}$ im Vergleich mit $(1 / R)^{2}$.

werden: Unter der Annahme, dass das $1 / f$-Rauschen im Wesentlichen durch den Tunnelprozess über die MgO-Tunnelbarriere verursacht wird, können magnetische Verunreinigungen in der Barriere, welche bis zu einem Feld von 1000 Oe in den Transportprozess involviert sind, ausgeschlossen werden. Würde ein Teil der Elektronen über lokalisierte Zwischenzustände mit einem magnetischen Moment tunneln, hätte die Ausrichtung des magnetischen Moments durch das externe Feld einen Einfluss auf die Rauschamplitude [136]. Weiterhin kann die Bewegung von Domänenwänden als Ursache für das Rauschen ausgeschlossen werden, da die Probe für $H=1024$ Oe vollständig gesättigt ist [166].

Die Temperatur hat im Gegensatz zum äußeren Magnetfeld einen Einfluss auf den Parameter $a_{U}$. Ein Maximum befindet sich in der Nähe der Temperatur, bei welcher der Widerstand ein Minimum hat. Wird wiederum angenommen, dass der Tunnelprozess die Hauptursache für das Rauschen darstellt, so kann das Maximum von $\left\langle a_{U}\right\rangle$ über das Temperaturverhalten des Widerstandes qualitativ verstanden werden. Der Parameter $a_{U}$ ist über Gleichung 6.4 mit der spektralen Rauschleistungsdichte $S_{U}$ verknüpft und somit proportional zu $(\delta R / R)^{2}$, wobei $\delta R$ die Abweichung vom Mittelwert $\bar{R}$ bezeichnet. Der quantenmechanische Tunneleffekt, welcher als Hauptursache für das Rauschen angesehen wird, ist aber temperaturunabhängig (s. Kap. 3.1.4). Daher kann angenommen werden, dass $\delta R$ ebenfalls temperaturunabhängig und somit konstant ist. Demnach würde die Temperaturabhängigkeit von $\left\langle a_{U}\right\rangle$ nur über die des Widerstandes $R$ kontrolliert, welche bekannt ist. Ein Vergleich von $\left\langle a_{U}\right\rangle$ mit $1 / R^{2}$ ist in Abbildung 6.7 gegeben. Es zeigt sich eine gute Übereinstimmung. Im Umkehrschluss kann demnach der Tunnelprozess als dominante Ursache für das Rauschen angesehen werden.

Die Größenordnung des normierten Rauschens $\left\langle a_{U}\right\rangle \approx 10^{-27} \mathrm{~m}^{3}$ ist vergleichbar mit Werten für reine, epitaktische Manganatproben. Auf STO-Substraten gewachsene und somit stark verspannte Filme zeigen ein hohes Rauschniveau $\left(a_{U} \approx 10^{-24} \mathrm{~m}^{3}\right)$, während unverspannte Filme, gewachsen auf $\mathrm{NdGaO}_{3}$-Substraten, eine deutlich niedrigere Rauscham- 
plitude aufweisen $\left(a_{U} \approx 10^{-30} \mathrm{~m}^{3}\right)$. Die Tatsache, dass der in dieser Arbeit ermittelte Wert für die Nanokomposite zwischen den Werten für verspannte und unverspannte epitaktische Manganatproben liegt, deutet auf eine sehr hohe Qualität der Schicht und insbesondere der Grenzflächen. Weiterhin liefert die hier angefertigte systematische Rauschanalyse keinen Hinweis auf einen gestörten Bereich an der Grenzfläche LSMO/MgO.

\subsection{LSMO: $\mathrm{CeO}_{2}$ Nanokomposite}

Wie auch schon andere Arbeiten gezeigt haben, ist es möglich, statt MgO andere isolierende Materialien in Nanokompositen zu verwenden [31]. Im Folgenden wird auf das $\mathrm{CeO}_{2}$-dotierte System näher eingegangen, welches unter anderem aufgrund der nahezu perfekten Gitterpassung an das Manganat und der geringeren Barrierenhöhe im Gegensatz zu MgO ein geeigneter Kandidaten als Barrierenmaterial ist.

Teile der vorgestellten Ergebnisse (Probenpräparation, Röntgencharakterisierung und Magnetisierungsmessungen) wurden in Zusammenarbeit mit Hamish Gordon, Cambridge, im Rahmen eines Studentenaustausches gewonnen.

$(\mathrm{LSMO})_{1-x}:\left(\mathrm{CeO}_{2}\right)_{x}$ Proben mit $0,05 \leq x \leq 0,5$ werden mittels MAD-Technik auf (001)-orientierten $\mathrm{Al}_{2} \mathrm{O}_{3}$-Substraten deponiert. Die Schichtdicke beträgt dabei für alle Filme ca. $70 \mathrm{~nm}$.

\subsubsection{Strukturanalyse}

Die strukturelle Untersuchung aller Proben wird mittels Röntgendiffraktometrie und REM-Aufnahmen vorgenommen. Abbildung 6.8 a) zeigt ein typisches Ergebnis einer Röntgenuntersuchung an der $x=0,2$ Probe. Unter Berücksichtigung des AluminiumProbenhalters können alle auftretenden Reflexe indiziert werden, wobei keine weiteren Phasen neben den beiden identifizierten Systemen LSMO und $\mathrm{CeO}_{2}$ einbezogen werden müssen. Sowohl für LSMO als auch für $\mathrm{CeO}_{2}$ treten mehrere Orientierungen senkrecht zur Schicht auf, wobei neben dem Substrat-Reflex der LSMO-(111)-Reflex die höchste Intensität aufweist. Aufgrund der passenden Gitterbeziehung treten die Reflexe LSMO(011) und $\mathrm{CeO}_{2}$-(002) bei einem identischen Winkel auf. Der $\mathrm{CeO}_{2}-(111)$-Reflex hingegen überlappt mit keinem weiteren LSMO-Reflex und kann somit zur Analyse der chemischen Phasenseparation herangezogen werden. In Abbildung 6.8 b) ist die auf den LSMO (111)Reflex normierte Intensität des $\mathrm{CeO}_{2}$-(111)-Reflexes für unterschiedliche $\mathrm{CeO}_{2}$-Anteile aufgetragen. Während für die Probe mit $x=0,05$ keine Intensität gemessen werden kann, steigt sie im Weiteren systematisch mit dem $\mathrm{CeO}_{2}$-Gehalt an. Weiterhin tritt das Maximum für ansteigenden $\mathrm{CeO}_{2}$-Gehalt bei kleineren Winkeln auf, die Gitterkonstante senkrecht zum Substrat vergrößert sich somit.

REM-Aufnahmen der Oberflächen erlauben die Abschätzung der lateralen Korngröße. Abbildung 6.9 zeigt ein typisches Ergebnis für die $x=0,05$ Probe. Deutlich sind vorwiegend dreieckige Strukturen zu erkennen, welche eine mittlere Größe von ca. $50 \mathrm{~nm}$ aufweisen. Die Korngröße aller weiteren Proben liegt im Bereich zwischen 20 und $50 \mathrm{~nm}$, 

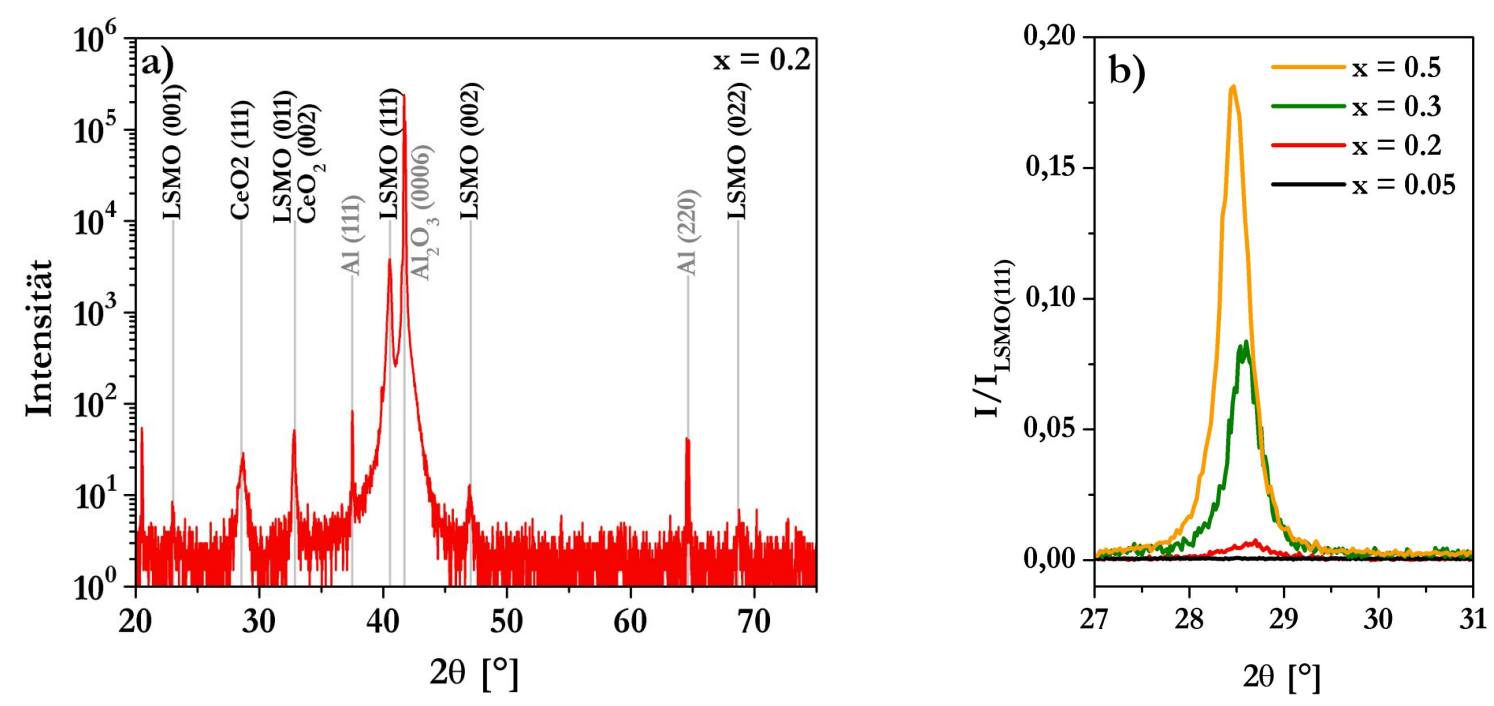

Abbildung 6.8. Röntgenstrukturanalyse einer LSMO: $\mathrm{CeO}_{2}$ Nanokompositschicht mit einem $\mathrm{CeO}_{2}$-Anteil von $20 \%$. b) Normierte Intensität des $\mathrm{CeO}_{2}$ (111)-Reflexes für unterschiedliche $\mathrm{CeO}_{2}$-Anteile.

ohne eine systematische Abhängigkeit von $x$ zu zeigen. Die Probe mit $x=0,2$ hat dabei mit ca. $20 \mathrm{~nm}$ die kleinste mittlere Korngröße.

\subsubsection{Magnetotransport}

Die Temperaturabhängigkeit des auf den Metall-Isolator-Übergang normierten Widerstands ist für verschiedene $\mathrm{CeO}_{2}$-Anteile in Abbildung 6.10 a) dargestellt. Die Messungen erfolgen an unstrukturierten Filmen. Die Proben mit $x \leq 0,1$ zeigen ein ähnliches Verhalten, welches dem einer undotierten Probe gleicht. Die Temperaturen maximalen Widerstands betragen jeweils ca. $360 \mathrm{~K}(x=0,05)$, bzw. $370 \mathrm{~K}(x=0,1)$. Für tiefe Temperaturen ist ein leichter Anstieg des Widerstands mit abnehmender Temperatur zu beobachten. Mit ansteigendem $\mathrm{CeO}_{2}$-Gehalt befindet sich das Maximum im Widerstandsverlauf bei immer tieferen Temperaturen. Dabei sind die Maxima deutlich breiter als für die Proben mit $x \leq 0,1$. Auch der Anstieg des Widerstands bei tiefen Temperaturen wird mit zunehmendem $\mathrm{CeO}_{2}$-Anteil dominanter. So ist der Widerstand der $x=0,5$ Probe bei tiefen Temperaturen am höchsten und nicht am Metall-Isolator-Übergang.

Der spezifische Widerstand der Proben bei $T=50 \mathrm{~K}$ als Funktion des $\mathrm{CeO}_{2}$-Gehalts $x$ ist in Abbildung 6.10 b) dargestellt. Ein Vergleich bei $T=50 \mathrm{~K}$ wird einerseits aufgrund der konzentrationsabhängigen Metall-Isolator-Übergangstemperatur, andererseits aufgrund des ansteigenden Widerstands zu tiefen Temperaturen hin gewählt. Die Einflüsse beider Effekte erscheinen bei $T=50 \mathrm{~K}$ vergleichsweise gering. Die spezifischen Widerstände für $x=0,05$ und $x=0,1$ sind vergleichbar, wohingegen der spezifische Widerstand für $x=0,2 \mathrm{um}$ mehr als eine Größenordnung höher ist. Der spezifische Widerstand für $x=0,3$ wiederum ist gegenüber $x=0,2$ leicht verringert, für $x=0,4$ steigt er auf seinen Maximalwert von ca. $2 \Omega \mathrm{cm}$ an. Aus experimentellen Gründen konnte der spezifische Widerstand für $x=0,5$ nicht ermittelt werden. 


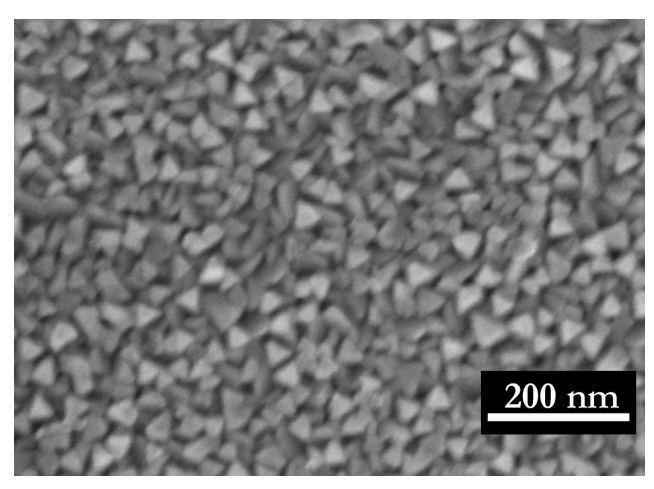

Abbildung 6.9. REM-Aufnahme der Oberfläche der (LSMO) $)_{0,95}:\left(\mathrm{CeO}_{2}\right)_{0,05}-$ Probe.
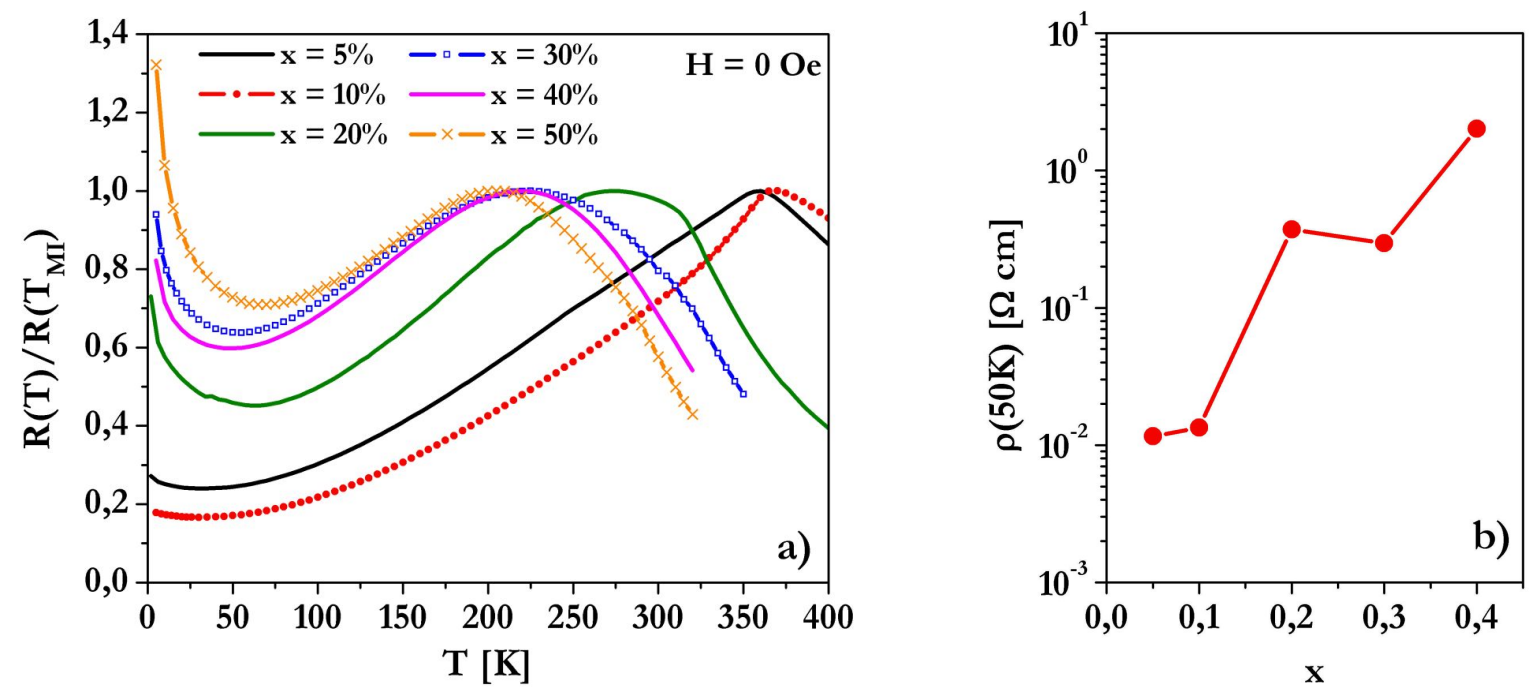

Abbildung 6.10. a) Temperaturabhängigkeit des Widerstandes für (LSMO) $)_{1-x}:\left(\mathrm{CeO}_{2}\right)_{x}$, $0,05 \leq x \leq 0,5$, normiert auf den Widerstand am jeweiligen Metall-Isolator-Übergang. $\mathrm{b}$ ) spezifischer Widerstand bei $T=50 \mathrm{~K}$ als Funktion des $\mathrm{CeO}_{2}$-Gehalts $x$.
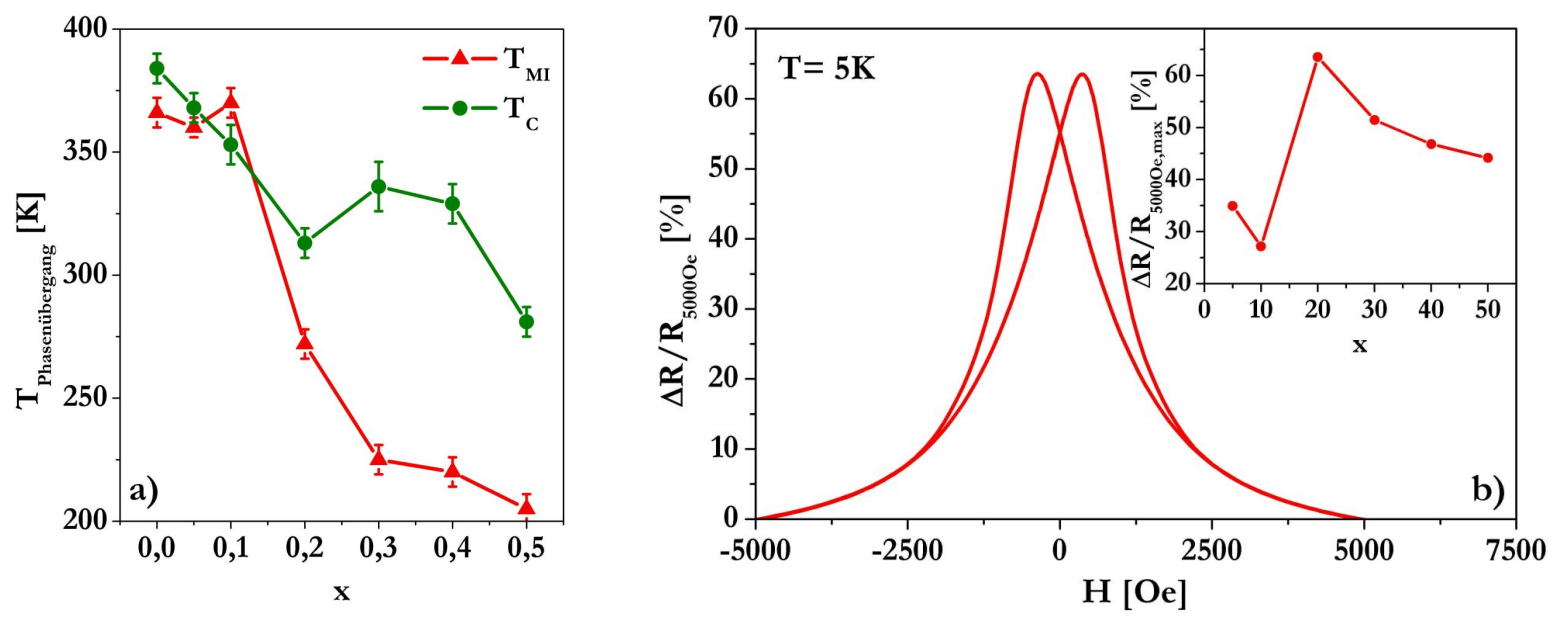

Abbildung 6.11. a) Phasenübergangstemperaturen als Funktion des $\mathrm{CeO}_{2}$-Gehalts $x$. b) Magnetowiderstand bei $T=5 \mathrm{~K}$ für $(\mathrm{LSMO})_{0,8}:\left(\mathrm{CeO}_{2}\right)_{0,2}$. Einsatz: Magnetowiderstand bei $T=5 \mathrm{~K}$ als Funktion des $\mathrm{CeO}_{2}$-Gehalts $x$. 
Aus temperaturabhängigen Magnetisierungsmessungen kann die Curie-Temperatur der jeweiligen Proben gewonnen werden. Für $x \leq 0,1$ stimmen $T_{C}$ und $T_{M I}$ in etwa überein, siehe Abbildung 6.11a). Für höheres $x$ zeigt $T_{C}$ eine abnehmende Tendenz, bleibt aber immer deutlich größer als $T_{M I}$. Für $x=0,5$ ist beispielsweise $T_{M I} \approx 205 \mathrm{~K}$, aber $T_{C} \approx 280 \mathrm{~K}$.

Der Magnetowiderstand bei $T=5 \mathrm{~K}$ ist für $x=0,2$ in Abbildung 6.11 b) dargestellt. Im Gegensatz zu Kapitel 6.1 wird der Magnetowiderstandseffekt auf den Widerstandswert bei $H=5000$ Oe normiert, da das Sättigungsfeld deutlich höher liegt. Unter Verwendung dieser Definition ergibt sich für $x=0,2$ ein maximaler Magnetowiderstandseffekt von mehr als $63 \%$ bei $T=5 \mathrm{~K}$. Das Feld maximalen MRs stimmt dabei mit dem Koerzitivfeld der Magnetisierung überein. Bei diesem Feld ist die Unordnung der magnetischen Momente maximal und die spinabhängige Tunnelwahrscheinlichkeit entsprechend am geringsten. Der Einsatz in Abbildung 6.11 zeigt den maximalen Magnetowiderstand bei $T=5 \mathrm{~K}$ als Funktion des $\mathrm{CeO}_{2}$-Gehalts $x$. Für $x=0,2$ zeigt sich ein deutliches Maximum. Dennoch sind auch die Magnetowiderstandswerte der übrigen Proben sehr hoch, beispielsweise mehr als $45 \%$ für $x=0,5$.

\subsubsection{Einfluss der Strukturgröße}

Aufgrund der Struktur der Nanokomposite mit Körnern der Größenordnung $50 \mathrm{~nm}$ ist es a priori nicht klar, ob die beobachteten physikalischen Eigenschaften intrinsisch sind für jedes einzelne System Korn - Isolator oder ob sie aus einer Mittelung über alle beteiligten Körner resultieren. Um dieser Frage nachzugehen, wird für die Probe $x=0,05$ die Anzahl der in den Transportprozess involvierten Körner systematisch reduziert. Hierzu werden Mikrostrukturen, wie in Kapitel 5.5 beschrieben, auf der Probe definiert. Einerseits findet Struktur 2 mit einer Steglänge von $20 \mu \mathrm{m}$ und -breite von $2 \mu \mathrm{m}$ Anwendung, im Folgenden Mikrostruktur genannt, andererseits wird die FIB-Struktur verwendet, welche ca. $1 \mu \mathrm{m}$ lang und ca. $300 \mathrm{~nm}$ breit ist. Die Breite des jeweiligen Steges entspricht somit ca. 20 Körnern für die Mikrostruktur, bzw. ungefähr sechs Körnern für die FIB-Struktur.

Die Temperaturabhängigkeit des normalisierten Widerstandes der globalen Messung (unstrukturiert), der Mikrostruktur und der FIB-Struktur wird in Abbildung 6.12 a) mit der normalisierten Magnetisierung verglichen. Die Curie-Temperatur stimmt im Wesentlichen mit der Metall-Isolator-Übergangstemperatur für die globale Messung überein. Wird die Anzahl der untersuchten Körner reduziert, so sinkt die Temperatur maximalen Widerstandes systematisch. Für die globale Messung beträgt sie $360 \mathrm{~K}$, für die Mikrostruktur $280 \mathrm{~K}$ und für die FIB-Struktur nur $235 \mathrm{~K}$. Auffallend ist die Schulter im Widerstandsverlauf der FIB-Struktur in der Nähe der Curie-Temperatur der Probe.

Der Magnetowiderstand bei $T=5 \mathrm{~K}$ für die drei oben beschriebenen Messgeometrien ist in Abbildung 6.12 b) dargestellt. Auffällig ist die deutliche Verbreiterung der Hysterese des MRs für die FIB-Struktur, welche verrauschter ist als die beiden anderen Kurven. Der maximale Magnetowiderstand variiert nur gering mit der Strukturgröße: Er beträgt 33\% für die globale Messung, 36\% für die Mikrostruktur und 32\% für die FIB-Struktur. 

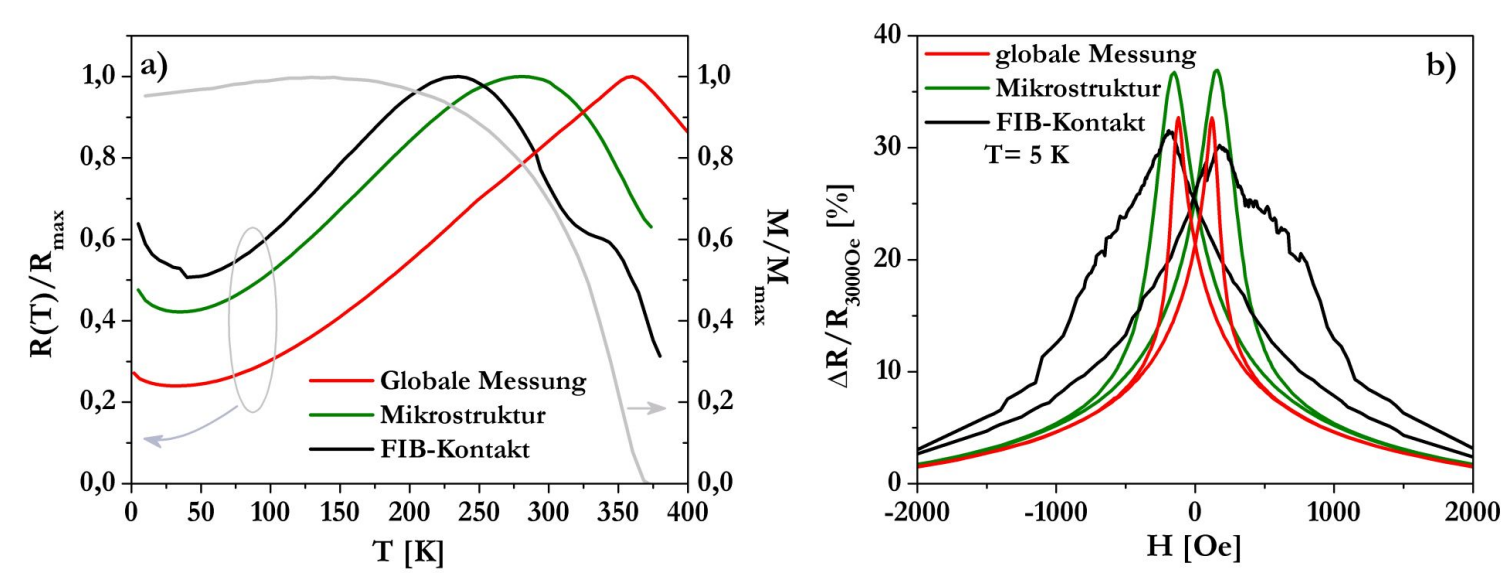

Abbildung 6.12. (LSMO $)_{0,95}:\left(\mathrm{CeO}_{2}\right)_{0,05}$ : a) Temperaturabhängigkeit des Widerstandes für unterschiedliche Systemgrößen. b) Magnetowiderstand der Strukturen bei $T=5 \mathrm{~K}$.

\section{Strominduzierte Effekte}

Die geringe Strukturbreite von $300 \mathrm{~nm}$, kombiniert mit der Schichtdicke von $70 \mathrm{~nm}$, bietet die Möglichkeit, hohe Stromdichten durch den Kontakt zu treiben und so gegebenenfalls strominduzierte Effekte wie Magnetisierungsschalten zu beobachten. Abbildung 6.13 zeigt den Widerstand bei $T=30 \mathrm{~K}$ ohne äußeres Magnetfeld als Funktion der Stromstärke im Bereich $20 \mu \mathrm{A} \leq|\mathrm{I}| \leq 40 \mu \mathrm{A}$. Es zeigt sich, dass der Widerstand zu großen Stromstärken hin abnimmt, also eine nichtlineare Beziehung zwischen Strom und Spannung vorliegt. Zusätzlich treten für beide Polaritäten Sprünge im Widerstand auf, welche hysteretisches Verhalten aufweisen. Wird die Stromstärke beispielsweise von kleinen, positiven Werten kommend erhöht (Abb. 6.13 b), so ergibt sich eine Stufe bei $I_{\text {hin }}^{\text {pos }}=35 \mu \mathrm{A}$. Ausgehend von hohen, positiven Stromstärken springt der Widerstand aber erst bei $I_{r c k}^{p o s}=26 \mu \mathrm{A}$ auf die alte Widerstandskurve zurück. Dieses Verhalten wird auch für negative Stromstärken beobachtet [Abb. 6.13a)], wobei hier die Widerstandswerte für kleine Stromstärken leicht voneinander abweichen. Die kritischen Stromstärken betragen $I_{h i n}^{n e g}=37 \mu \mathrm{A}$, bzw. $I_{r c k}^{n e g}=27 \mu \mathrm{A}$. Die sich aus diesen Stromstärken ergebenden Stromdichten liegen in der Größenordnung von $J_{C} \approx 1,5 \cdot 10^{5} \mathrm{~A} / \mathrm{cm}^{2}$. Dieses Verhalten ist aber nur temporär zu beobachten und tritt bei anschließenden Messungen des gleichen Kontaktes nicht mehr auf.

\subsubsection{Diskussion}

\section{Struktur}

In den Röntgenanalysen der Proben zeigen sich neben der dominanten (111)-Orientierung des LSMO mehrere Wachstumsrichtungen geringerer Intensität senkrecht zum Substrat. Es kann auf polykristallines, vorwiegend (111)-orientiertes Wachstum geschlossen werden. Die Indizierung aller auftretenden Reflexe neben dem Substrat und Probenhalter gelingt dabei unter Berücksichtigung der Phasen LSMO und $\mathrm{CeO}_{2}$, was auf eine chemische Phasenseparation hindeutet, wie sie auch schon früher beobachtet wurde [30,78, 192,. Die 

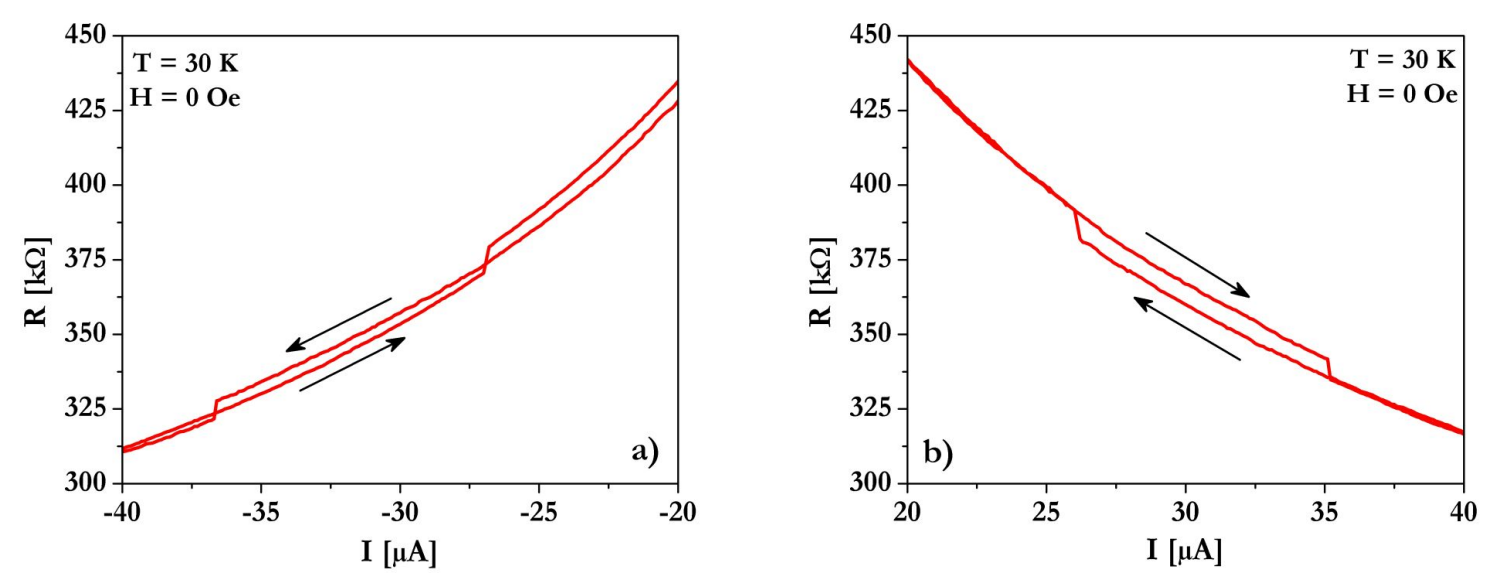

Abbildung 6.13. (LSMO $)_{0,95}:\left(\mathrm{CeO}_{2}\right)_{0,05}$ : Stromabhängigkeit des FIB-Kontaktes bei $T=$ $30 \mathrm{~K}$ ohne äußeres Magnetfeld.

Phasenseparation wird ebenfalls bestätigt durch die mit dem $\mathrm{CeO}_{2}$-Gehalt ansteigende Intensität des $\mathrm{CeO}_{2}$-(111)-Reflexes [Abb. 6.8 b)]. Allerdings vergrößert sich mit zunehmendem $x$ der Gitterparameter der $\mathrm{CeO}_{2}$-Phase. Ryan et al. haben gezeigt, dass es bei einer La-Dotierung von $\mathrm{CeO}_{2}$ zu einer Gitteraufweitung von bis zu $4 \%$ kommt [193]. Auch Stingl vermutet die Ausbildung einer La-dotierten $\mathrm{CeO}_{2}$-Phase bei seinen Untersuchungen zur Präparation von $\mathrm{La}_{2 / 3} \mathrm{Ce}_{1 / 3} \mathrm{MnO}_{3}|77|$. Dies legt die Interpretation nahe, dass hier zumindest in Teilen eine La-Dotierung des $\mathrm{CeO}_{2}$ vorliegt und es zur Aufweitung des Gitters kommt. Die Dotierung scheint dabei mit $x$ anzusteigen, da für $x=0,5$ die größte Aufweitung des Gitters ( $a=0,543 \mathrm{~nm})$ um 0,4\% zu beobachten ist. Nach Ryan et al. [193] entspricht diese Aufweitung einer La-Dotierung von ca. $10 \%$.

Aus der obigen Hypothese des La-dotierten $\mathrm{CeO}_{2}$ ergibt sich die Folgerung, dass zumindest Teile des LSMO La-defizitär sein müssen. Im Röntgendiffraktogramm gibt es jedoch keine Hinweise darauf. Weiterhin kann mittels Röntgenanalyse keine Aussage darüber getroffen werden, ob das isolierende, La-dotierte $\mathrm{CeO}_{2}$ die einzelnen LSMO-Körner tatsächlich trennt.

\section{Magnetotransport}

Die hohen Phasenübergangstemperaturen (360-370 K) besonders für $x \leq 0,1$ deuten auf eine hohe Qualität der Proben. Für $x \leq 0,1$ liegen sie nur wenig unterhalb der Literaturwerte für reines LSMO. Hiermit lässt sich auch die chemische Phasenseparation bestätigen. Unter Berücksichtigung der obigen Hypothese (mit $x$ ansteigende La-Dotierung des $\mathrm{CeO}_{2}$ ) kann die Abnahme der Phasenübergangstemperatur durch ein ansteigendes La-Defizit im LSMO verstanden werden. Dies steht mit der Tatsache in Einklang, dass die Metall-Isolator-Temperatur für $x \geq 0,2$ immer kleiner ist als die Curie-Temperatur. So könnte das La-Defizit in der LSMO-Phase an der Oberfläche der Körner, also an der Grenzfläche zum Isolator, größer sein und somit einen größeren Einfluss auf den Transport haben, während das Innere des Korns eine höhere Curie-Temperatur aufweist als der Oberflächenbereich (core-shell model). Dabei bleibt ungeklärt, warum die La-Dotierung 
des $\mathrm{CeO}_{2}$ erst für $x \geq 0,2$ einsetzt und für kleinere Werte kein Effekt zu beobachten ist.

Der spezifische Widerstand der Proben zeigt eine deutliche Abhängigkeit vom $\mathrm{CeO}_{2^{-}}$ Gehalt, besonders in der Umgebung von $x=0,2$. Dabei kann der im Vergleich zu $x=0,3$ höhere spezifische Widerstand für $x=0,2$ vermutlich durch die kleinere Korngröße von 20 nm für $x=0,2$ erklärt werden. Der hohe Anstieg um mehr als eine Größenordnung in Bezug auf $x=0,1$ kann möglicherweise mit einem Perkolationsübergang des LSMO gedeutet werden, allerdings erscheint der kritische $\mathrm{CeO}_{2}$-Gehalt sehr gering. Üblicherweise werden Perkolationsübergänge einer metallischen Phase in Systemen (Metall) ${ }_{1-x}$ :(Isolator) ${ }_{x}$ in einem Konzentrationsbereich um $x=0,7$ beobachtet [194]. Der große Unterschied in den spezifischen Widerständen von $x=0,1$ und $x=0,2$ ist womöglich ebenfalls auf die Differenz in den mittleren Korngrößen zurückzuführen.

Von besonderer Bedeutung für dieses System ist der hohe Wert des Magnetowiderstandes. Dieser liegt für alle untersuchten $\mathrm{CeO}_{2}$-Anteile $x$ oberhalb von $20 \%$ und für $x=0,2$ bei über $60 \%$. Ein Vergleich mit der Literatur zeigt, dass dieser Wert deutlich über den bisher erzielten Werten für das LSMO:CeO- $\mathrm{C}_{2}$-System liegt. Balcells et al. [30] zeigen einen maximalen LFMR von $10 \%$ bei tiefen Temperaturen für $x=0$, 8, im System LCMO:CeO ${ }_{2}$ zeigen Yao et al. 192 einen LFMR von ungefähr 25\% für $x=0,65$. Die Ursache für den maximalen LFMR kann in einem eventuellen Perkolationsübergang bei $x=0,2$ gesehen werden. Nahe des Perkolationsübergangs sind alle Körner mit einer isolierenden Schicht bedeckt, es kommt zum spinabhängigen Tunnelprozess. Wird die Breite der isolierenden Schicht vergrößert, werden Spin-flip-Prozesse wahrscheinlicher und der LFMR nimmt ab. Wie aber schon oben diskutiert, ist ein Perkolationsübergang für einen solch geringen $\mathrm{CeO}_{2}$-Gehalt sehr unwahrscheinlich. Vielmehr kann das Maximum auch durch zwei konkurrierende Prozesse erklärt werden: Die einzelnen Körner werden mit zunehmendem Anteil der isolierenden Phase entkoppelt, was den LFMR zunächst erhöht [30]. Unter Annahme des oben entwickelten core-shell-Modells ergibt sich für $x \geq 0,2$ jedoch ein zunehmendes La-Defizit an der Grenzfläche zum Isolator, entsprechend ist auch eine Verringerung der Spinpolarisation anzunehmen, woraus eine Abnahme des LFMR resultiert. Da ein Einsetzen der La-Dotierung für $x=0,2$ aus den strukturellen Ergebnissen abgeleitet werden kann, erscheint die Erklärung unter Annahme des core-shell-Modells wahrscheinlicher als das Perkolationsszenario. Eine direkte Untersuchung der Grenzfläche beispielsweise mit element-selektiver TEM-Analyse ist zur Bestätigung bzw. Negierung der Hypothesen notwendig.

\section{Einfluss der Strukturgröße}

Durch die systematische Reduktion der Anzahl der in den Transportprozess involvierten Körner kann geschlossen werden, ob die physikalischen Eigenschaften, welche durch globale Messungen identifiziert werden, durch die Mittelung über viele Körner bzw. Grenzflächen Korn-Isolator bestimmt werden oder ob sie für jedes einzelne System intrinsisch sind. Für den ersten Fall sollte eine Einschränkung des Strompfades auf wenige Körner eine deutliche Änderung des Widerstandsverlaufes hervorrufen, während im zweiten Fall kaum Abweichungen vom Verlauf der unstrukturierten Probe zu beobachten sein sollten. 
Der temperaturabhängige Widerstandsverlauf für die verschiedenen Strukturgrößen lässt zunächst vermuten, dass die Metall-Isolator-Übergangstemperatur systematisch mit der Strukturgröße abnimmt, da die Temperatur maximalen Widerstandes dieses Verhalten aufweist. Die Schulter in der FIB-Messung weist aber darauf hin, dass der intrinsische Phasenübergang nahe der Curie-Temperatur lediglich durch einen weiteren überdeckt wird. Dieser zweite Übergang wird möglicherweise durch einen gestörten Bereich des LSMO an der Grenzfläche zum Isolator verursacht, wie er bereits für das MgO-dotierte System vermutet wird. Die Breite des Maximums deutet auf eine breite Verteilung dieses zweiten Übergangs hin, welche im Widerstandsverlauf der Mikrostruktur eine gegebenenfalls auftretende Schulter überdecken könnte.

In der Messung des LFMR bei $T=5 \mathrm{~K}$ [Abb. 6.12 b)] ist zunächst die breite Form der Hysterese für die FIB-Struktur auffällig. Da die Koerzitivfelder ebenso wie die Sättigungsfelder mit Verringerung der Strukturbreite systematisch ansteigen, kann auf einen Geometrie-Effekt geschlossen werden. Die Breite des FIB-Kontaktes liegt nur knapp oberhalb der Größenordnung einer Domänenwand (ca. $50 \mathrm{~nm}$ [195]), s.d. die Anzahl der Domänen senkrecht zum Stromstreifen stark eingeschränkt ist. Folglich gewinnen PinningEffekte an den Rändern immer mehr Einfluss [196], wodurch die Koerzitiv- und Sättigungsfelder ansteigen. Zur Überprüfung dieser Vermutungen müssten lokal aufgelöste Magnetisierungsmessungen durchgeführt werden, was jedoch bei solchen Strukturgrößen eine große Herausforderung darstellt.

Der maximale LFMR bei $T=5 \mathrm{~K}$ ist nahezu unabhängig von der Strukturgröße. Aus dieser Tatsache lässt sich nach einem einfachen Modell von Wu et al. |197| schließen, dass die Verteilung der Barriereneigenschaften (Breite und Höhe) sehr schmal ist. Wäre die gaußförmige Verteilung mit der Breite $\sigma$ sehr weit [s. Abb. 6.14 b) für $\sigma=4$ ], so würde der Strom filamentartig über Pfade fließen, welche hauptsächlich dünne, spinerhaltende Barrieren enthalten. Eine Verringerung der Strukturgröße hätte demnach einen maßgeblichen Einfluss auf diese Pfade, in welche nun vermehrt dickere Barrieren mit nicht spinerhaltenden Eigenschaften aufgenommen werden müssen. Dies hätte entsprechend eine Verringerung des LFMR zur Folge [197]. Bei einer schmalen Verteilung der Barriereneigenschaften hingegen ist der Stromfluss und die Spin-Streuung über alle beteiligten Barrieren nahezu gleich [s. Abb. 6.14 a) für $\sigma=1$ ]. Eine Verringerung der Strukturgröße hat somit keinen Einfluss auf den LFMR. Im Umkehrschluss ergibt sich, dass aufgrund des ungeänderten LFMR zumindest bei tiefen Temperaturen eine schmale Verteilung der Barriereneigenschaften vorliegt. Hieraus ergibt sich, dass die spinabhängigen Eigenschaften bei tiefen Temperaturen intrinsisch sind für jede einzelne Grenzfläche LSMO-Isolator.

Aufgrund der geringen Breite der FIB-Struktur ist es möglich, hohe Stromdichten in diesem Kontakt zu erzeugen. Temporär werden strominduzierte Widerstandsänderungen beobachtet. Das allgemeine Abfallen des Widerstandes mit Erhöhung der Stromstärke ist vermutlich auf den Tunneleffekt zurückzuführen (s. Kap. 3.1). Eine mögliche Erklärung der Sprünge im Widerstandsverhalten ist das strominduzierte Magnetisierungsschalten (s. Kap. 3.3. Gegen diese Vermutung sprechen jedoch mehrere Tatsachen: Einerseits wäre die kritische Stromdichte mit $J_{C} \approx 1,5 \cdot 10^{5} \mathrm{~A} / \mathrm{cm}^{2}$ mindestens eine Größenordnung zu klein [109, 7]. Andererseits sollte der Effekt nur für eine Stromrichtung auftreten, was 

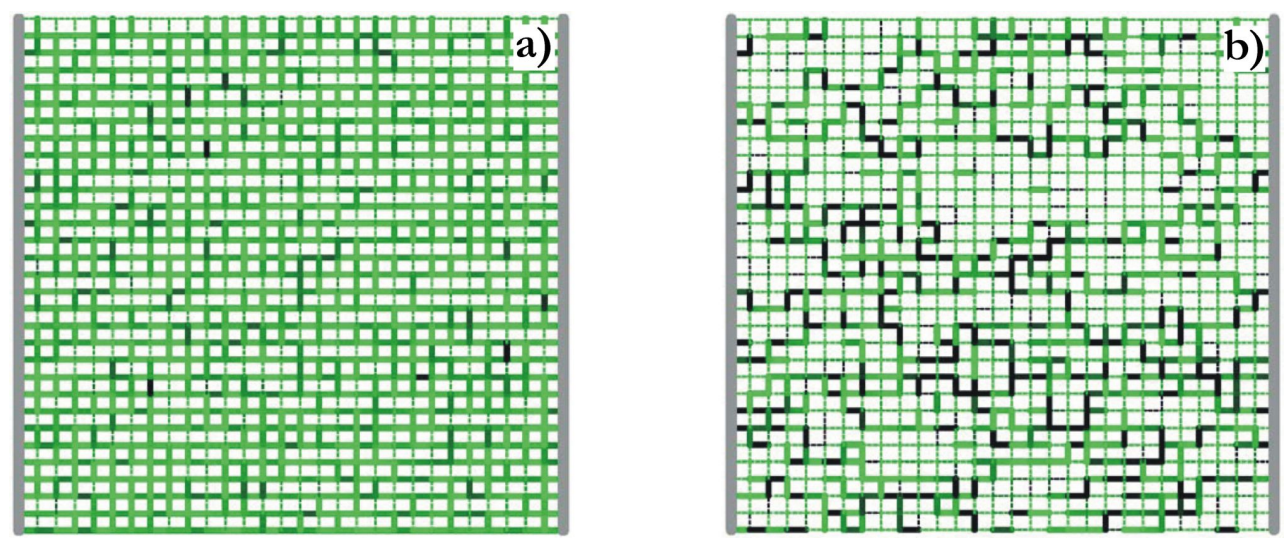

Abbildung 6.14. Modell von Wu et al:: Simulation der lokalen Stromverteilung in einem Netzwerk aus $30 \times 30$ Tunnelwiderständen. Schwarze Verbindungen repräsentieren stark spinerhaltenden Transport. a) schmale gaufförmige Verteilung der Barrierendicke $(\sigma=1)$; b) breite gaußförmige Verteilung der Barrierendicke $(\sigma=4)$ 197.

nicht der Fall ist. Eine Verifizierung durch die Magnetfeldabhängigkeit der kritischen Stromdichte ist nicht möglich, da der Effekt nicht reproduzierbar ist. Möglich ist auch eine Erklärung der abrupten Widerstandsänderungen über Effekte, wie sie beim spannungspulsinduzierten Widerstandsschalten in Perowskiten auftreten (s. Kap. 2.6). Aber auch dieser Effekt sollte nur für eine Stromrichtung zu beobachten sein und erscheint daher unwahrscheinlich. Zur genauen Interpretation fehlen letztlich genügend Daten, so dass über den Mechanismus keine endgültige Aussage getroffen werden kann. Dennoch bleibt festzuhalten, dass strominduzierte Widerstandsänderungen in lateral strukturierten Proben identifiziert werden konnten.

\subsection{Nanokristallines LSMO}

Zur Beobachtung weiterer, strominduzierter Widerstandseffekte ist eine Erhöhung der Stromdichten nötig. Eine Möglichkeit besteht in der Verminderung des Widerstandes des Systems, welches aber weiterhin einen LFMR aufweisen soll. Es liegt nahe, das isolierende Material in den Nanokompositen nicht weiter zu verwenden und auf reines, polykristallines LSMO zurückzugreifen. Hier dienen die Korngrenzen als Tunnelbarriere und ein LFMR tritt auf 27] (s.a. Kap. 3.4).

Eine $70 \mathrm{~nm}$ dicke LSMO-Probe wird mittels MAD-Technik auf einem (001)-orientierten $\mathrm{Al}_{2} \mathrm{O}_{3}$-Substrat präpariert. Die Probenpräparation wurde von Hamish Gordon, Cambridge, im Rahmen eines Studentenaustausches vorgenommen. Zur weiteren Charakterisierung der an dieser Schicht gefundenen Effekte wird zudem eine weitere Probe von ca. $50 \mathrm{~nm}$ Dicke unter sonst identischen Bedingungen deponiert, sowie eine epitaktische Schicht auf einem MgO-Substrat präpariert. Falls nicht weiter angegeben, beziehen sich alle Ergebnisse auf die $70 \mathrm{~nm}$ dicke Schicht. 

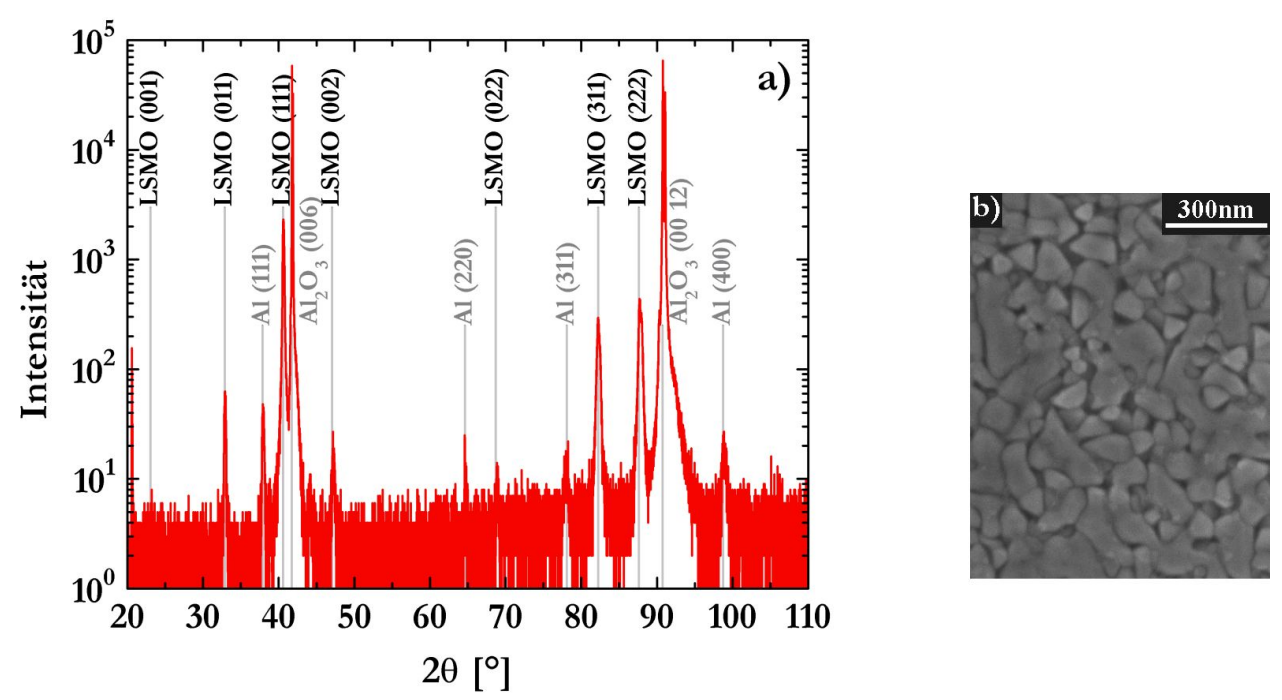

\begin{abstract}
Abbildung 6.15. a) Röntgenstrukturanalyse der LSMO-Schicht. b) REM-Aufnahme der Oberfläche. Der gezeigte Ausschnitt hat eine Größe von ca. $1 \mu \mathrm{m}$ Breite und ca. 1,5 $\mu \mathrm{m}$ Länge und entspricht somit ungefähr der Größe einer später auf der Probe definierten Mikrostruktur.
\end{abstract}

\title{
6.3.1 Strukturanalyse
}

Die Strukturanalyse wird mittels Röntgendiffraktometrie und REM vorgenommen. Im Röntgendiffraktogramm [Abb. 6.15]a)] treten abermals aufgrund der geringen Probenabmessungen auch Reflexe des Aluminium-Probenhalters auf, welche nicht weiter diskutiert werden. Alle weiteren Maxima können unter Berücksichtigung des $\mathrm{Al}_{2} \mathrm{O}_{3}$-Substrates und einer LSMO-Phase eindeutig indiziert werden. Neben dem Substrat zeigt der LSMO-(111)Reflex die höchste Intensität. Die durch seine Lage bestimmte Gitterkonstante ergibt sich zu $a=3,86 \AA$ A. Eine Scherrer-Analyse ergibt die mittlere Größe der kohärent streuenden Bereiche mit (111)-Orientierung zu $\tau \approx 45 \mathrm{~nm}$.

Abbildung 6.15b) zeigt die REM-Aufnahme der Probenoberfläche. Dabei entspricht die Größe des gezeigten Ausschnitts näherungsweise der eines später auf der Probe definierten Stromstreifens (s.u.). Wie bereits für die Komposite beobachtet, zeigen sich auch hier dreieckige Stukturen mit einer mittleren Größen von ca. $50 \mathrm{~nm}$. Daneben treten aber auch längliche Strukturen auf, welche eine größere laterale Ausdehnung von ca. $150 \mathrm{~nm}$ aufweisen.

\subsubsection{Magnetotransport}

An der unstrukturierten Probe durchgeführte Messungen ergeben eine Metall-IsolatorÜbergangstemperatur von $T_{M I} \approx 365 \mathrm{~K}$ und eine Curie-Temperatur, welche näherungsweise mit $T_{M I}$ übereinstimmt $\left(T_{C} \approx 375 \mathrm{~K}\right)$. Zur weiteren Analyse des elektrischen Widerstandes wird die Probe mikrostrukturiert, wobei zwei Modifikationen von Struktur 2 (s. Kap. 5.5.1 zur Anwendungen kommen. Die Länge beträgt für beide Strukturen 1,5 $\mu \mathrm{m}$, die Breite einerseits $0,9 \mu \mathrm{m}$, andererseits 2,2 $\mu \mathrm{m}$. Bei der Verwendung dieser Mikrostrukturen ist zu beachten, dass nur zwei Kontaktpads aufgebracht werden, auf denen jeweils eine 

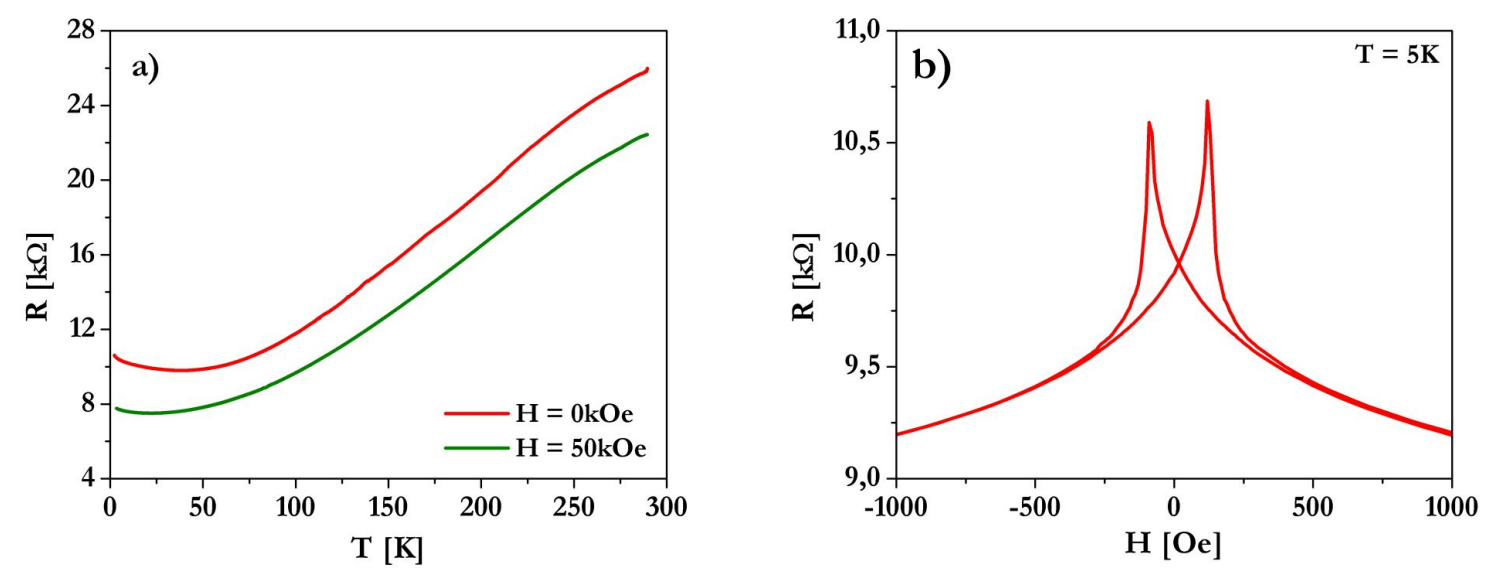

Abbildung 6.16. Magnetotransport der breiten Struktur: a) Temperaturabhängigkeit des elektrischen Widerstandes bei $H=0 \mathrm{kOe}$ und $H=50 \mathrm{kOe}(I=1 \mu \mathrm{A})$. b) Magnetfeldabhängigkeit des elektrischen Widerstandes bei $\mathrm{T}=5 \mathrm{~K}(I=1 \mu \mathrm{A})$.

Strom- und eine Spannungszuleitung kontaktiert sind. Eine echte Vier-Punkt-Messung wird demnach nicht vorgenommen.

Abbildung 6.16 a) stellt den Widerstand der breiten Mikrostruktur als Funktion der Temperatur mit und ohne äußerem Magnetfeld dar, jeweils gemessen mit $I=1 \mu \mathrm{A}$. Unterhalb von etwa $40 \mathrm{~K}$ zeigt sich für $H=0$ Oe ein leichter Anstieg des Widerstandes mit abnehmender Temperatur. Dieser Anstieg ist auch für $H=50 \mathrm{kOe}$ zu beobachten, allerdings setzt er erst unterhalb von ca. $25 \mathrm{~K}$ ein. Für beide Feldwerte wird sonst im zugänglichen Temperaturbereich ein metallisches Verhalten beobachtet. Das äußere Magnetfeld führt weiterhin für alle Temperaturen zu einer Verringerung des elektrischen Widerstandes. Die Abhängigkeit des Widerstandes vom äußeren Feld bei $T=5 \mathrm{~K}$ und $I=1 \mu \mathrm{A}$ zeigt Abbildung 6.16 b). Deutlich ist ein LFMR zu erkennen, welcher bei Normierung auf den Widerstandswert bei 1000 Oe einen Maximalwert von 16\% erreicht. Für die schmale Struktur ergeben sich ähnliche Werte (s.u.).

\subsubsection{Strominduzierte Effekte}

Um strominduzierte Effekte zu beobachten, wird der Widerstand bei fester Temperatur und festem, äußerem Magnetfeld als Funktion der Stromstärke gemessen. Die erhaltenen Daten werden zusätzlich in Strom-Spannungs-Kennlinien dargestellt. Bei der Interpretation dieser Kurven ist darauf zu achten, dass die systematisch variierte Größe die Stromstärke, nicht die Spannung ist. Alle gezeigten Messungen, für die jeweils ca. 15 min benötigt werden, laufen nach einem identischen Protokoll ab und werden immer mit folgender Farbkonvention dargestellt: Die Stromstärke wird zunächst von Null auf den maximalen, positiven Wert $I_{m a x}^{p o s}$ erhöht (rot), anschließend kontinuierlich auf den maximalen, negativen Wert $I_{m a x}^{\text {neg }}$ erniedrigt (grün), um letztlich wieder auf $I_{\max }^{\text {pos }}$ heraufgesetzt zu werden (schwarz). Aufgrund der Übersichtlichkeit wird in den Graphen der letzte Schritt nicht vollständig abgebildet. Dargestellt wird lediglich der Widerstandsverlauf von $I_{\max }^{\text {neg }}$ bis $I=0$. 


\section{Schmaler Kontakt}

Zunächst werden die Ergebnisse vorgestellt, welche an dem schmalen Kontakt (Breite $0,9 \mu \mathrm{m})$ gewonnen werden. Abbildung 6.17 a) zeigt den Verlauf des elektrischen Widerstandes bei $T=5 \mathrm{~K}$ und $H=5 \mathrm{kOe}$ als Funktion der Stromstärke im Bereich $|I| \leq 200 \mu \mathrm{A}$. Im Ausgangszustand (rote Kurve, $I \approx 0 \mu \mathrm{A}$ ) beträgt der Widerstand ca. $120 \mathrm{k} \Omega$, wobei er kontinuierlich mit Erhöhung der Stromstärke abnimmt und einen Wert von $70 \mathrm{k} \Omega$ bei $I=125 \mu \mathrm{A}$ annimmt. Bei $I_{C}=125 \mu \mathrm{A}$ fällt der Widerstand schlagartig auf einen Wert von $20 \mathrm{k} \Omega$ und bleibt für höhere Stromstärken nahezu konstant. Wird die Stromstärke nun wieder erniedrigt, so verbleibt der Widerstand auf einem Niveau von ca. $20 \mathrm{k} \Omega$. In der Umgebung von $I=0 \mu \mathrm{A}$ zeigt sich dabei ein kleines Maximum, der Widerstand steigt bis auf $25 \mathrm{k} \Omega$. Bei $I \approx-115 \mu \mathrm{A}$ ist ein abrupter Anstieg des Widerstands auf $73 \mathrm{k} \Omega$ zu beobachten. Für höhere negative Stromstärken nimmt der Widerstand kontinuierlich ab und beträgt $57 \mathrm{k} \Omega$ bei $I=-200 \mu \mathrm{A}$. Wird die Stromstärke wieder verringert, so steigt der Widerstand kontinuierlich an und erreicht bei $I \approx 0$ seinen Ausgangswert. Der nicht abgebildete Teil der Messung, also die erneute Bestimmung des Widerstandes für $0 \mu \mathrm{A} \leq \mathrm{I} \leq 200 \mu \mathrm{A}$, zeigt bis zum abrupten Abfall des Widerstandes den exakt identischen Verlauf. Der starke Widerstandseinbruch ist in diesem Fall aber erst bei $I_{C}=140 \mu \mathrm{A} z u$ beobachten. Weiterhin geht der Widerstand nicht auf den im ersten Teil der Messung beobachteten Wert von $20 \mathrm{k} \Omega$ zurück, sondern lediglich auf ca. $30 \mathrm{k} \Omega$.

Die Probe weist offensichtlich zwei unterschiedliche Widerstandszustände auf: einen niederohmigen, welcher durch Überschreiten einer, in diesem Falle positiven, kritischen Stromstärke erreicht werden kann, und einen hochohmigen Zustand, welcher der Ausgangszustand der Probe ist. Aus dem niederohmigen Zustand kommend kann der hochohmige Zustand durch eine ausreichend hohe, negative Stromstärke wiederhergestellt werden. Die beobachteten Widerstandsänderungen betragen dabei bis zu $700 \%$.

Die Abhängigkeit des Widerstandes von der Stromstärke lässt sich auch in einer StromSpannungs-Kennlinie darstellen [s. Abb. 6.17 b)]. Aus dem kontinuierlichen Abfall im hochohmigen Zustand ergibt sich eine nichtlineare Beziehung zwischen Stromstärke und Spannung, während im niederohmigen Zustand der nahezu von der Stromstärke unabhängige Widerstand zu einer linearen Abhängigkeit der Stromstärke von der abfallenden Spannung führt. Für hohe, positive Stromstärken liegt demnach Ohmsches Verhalten vor, für hohe, negative Stromstärken hingegen nichtlineares Verhalten.

Das bei $T=5 \mathrm{~K}$ und $H=5 \mathrm{kOe}$ beobachtete Verhalten des elektrischen Widerstandes als Funktion der Stromstärke ist nur in Teilen repräsentativ für das Verhalten der Probe. So wird zwar das Auftreten zweier verschiedener Widerstandszustände bei Verwendung ausreichend hoher Stromstärken für den gesamten zugänglichen Feldbereich bei $T=5 \mathrm{~K}$ beobachtet, aber insbesondere das Verhalten im niederohmigen Zustand zeigt starke Variationen. Ein Beispiel hierfür ist in Abbildung 6.18 a) dargestellt. Es zeigt den elektrischen Widerstand als Funktion der Stromstärke für $T=5 \mathrm{~K}$ in einem äußeren Feld von $H=40 \mathrm{kOe}$. Das Verhalten im hochohmigen Zustand entspricht qualitativ dem bei $H=5 \mathrm{kOe}$, die Absolutwerte des Widerstandes sind aufgrund des Magnetowiderstandseffekts verringert. Die Abhängigkeit des Widerstandes von der Stromstärke im 

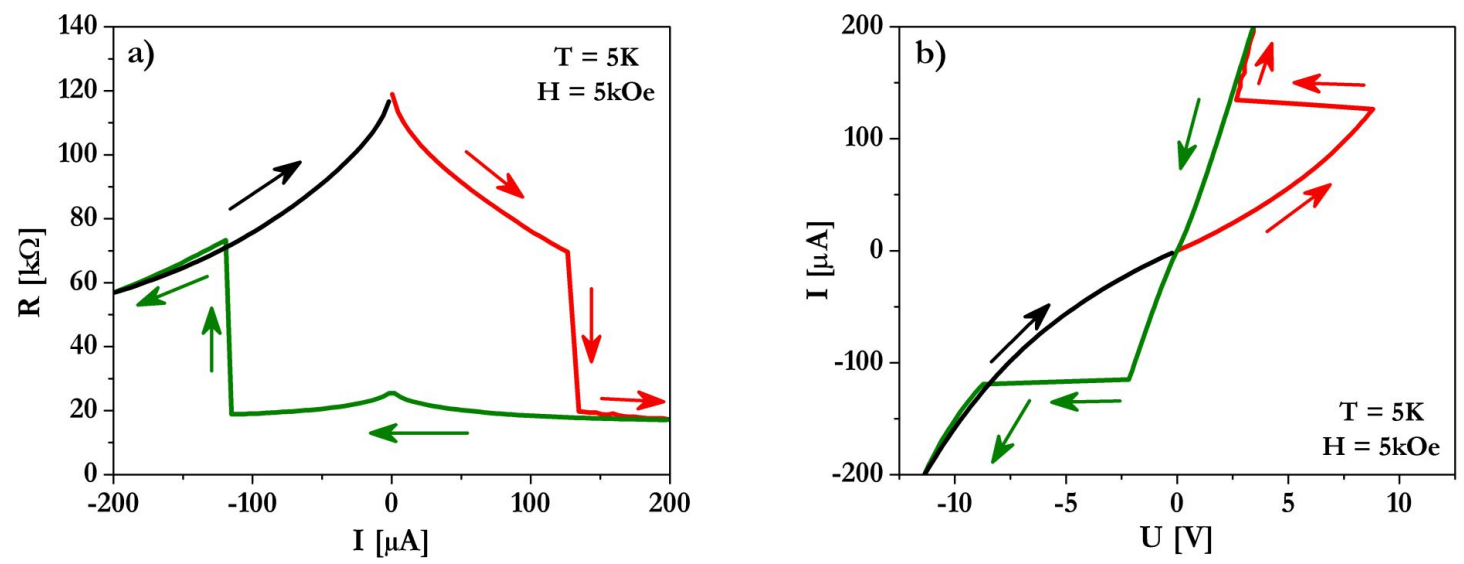

Abbildung 6.17. a) Widerstand des schmalen Kontakts als Funktion der Stromstärke bei $T=5 \mathrm{~K}$ und $H=5 \mathrm{kOe}$. b) Strom-Spannungs-Kennlinie der Daten nach a).
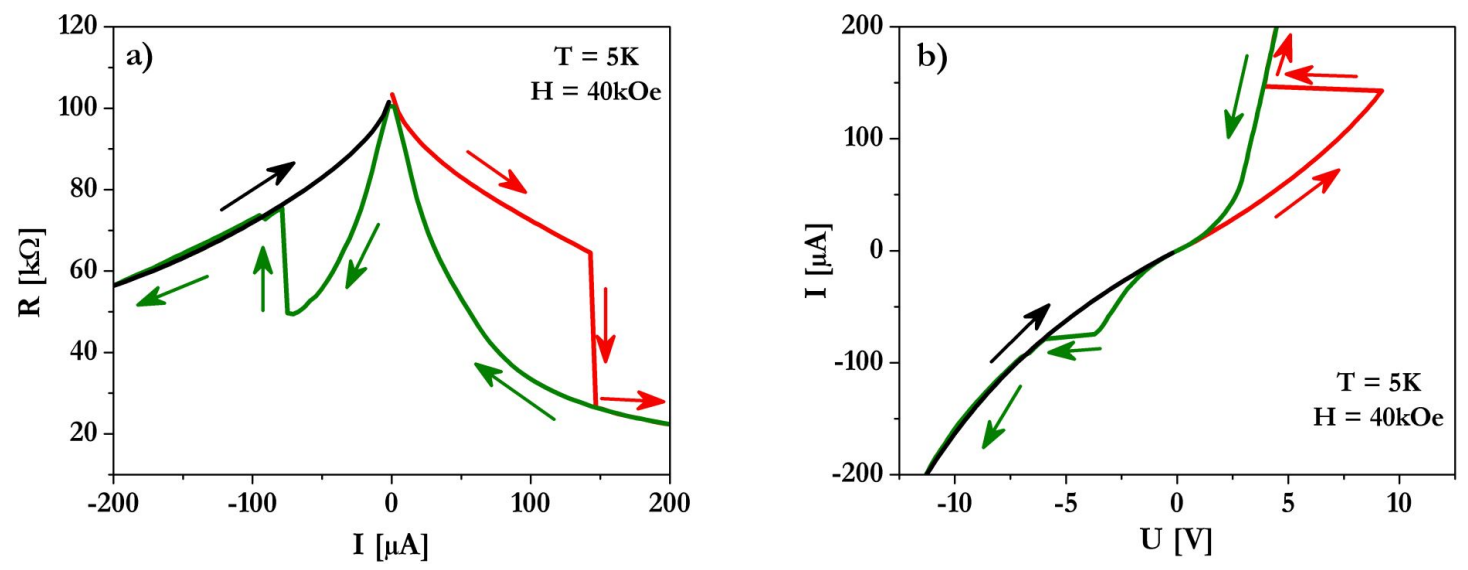

Abbildung 6.18. a) Widerstand des schmalen Kontakts als Funktion der Stromstärke bei $T=5 \mathrm{~K}$ und $H=40 \mathrm{kOe}$. b) Strom-Spannungs-Kennlinie der Daten nach a).
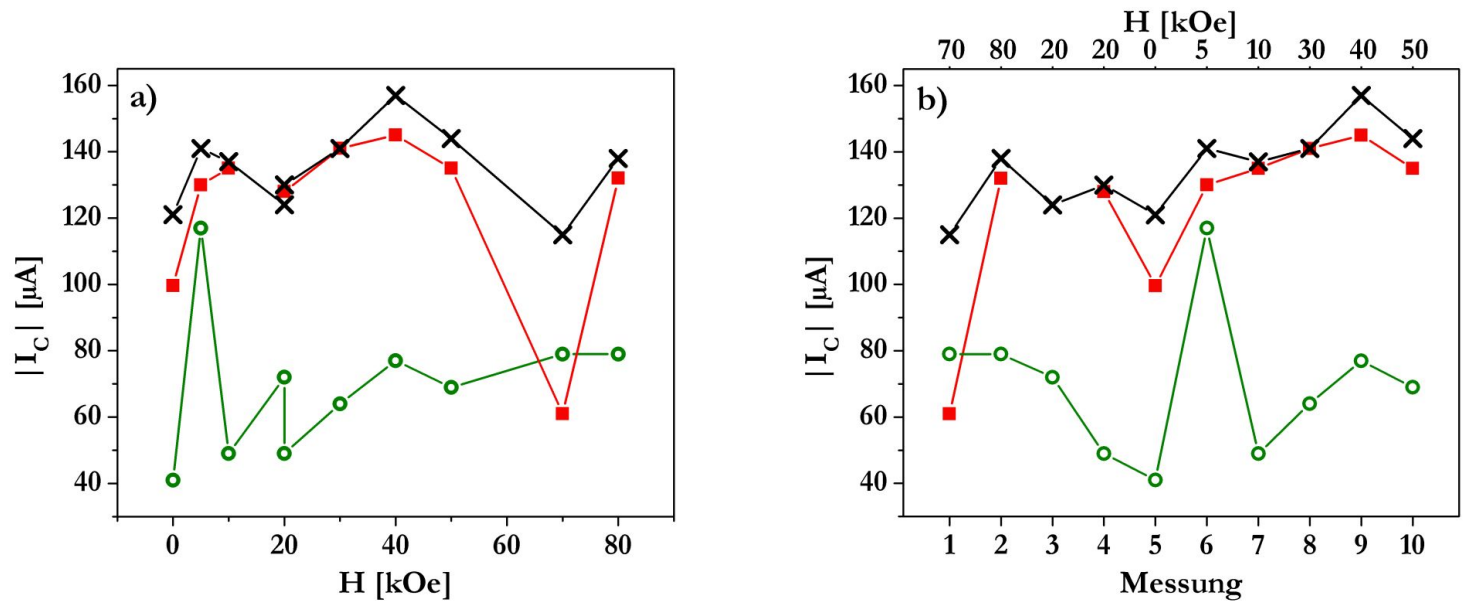

Abbildung 6.19. $\square: \mathrm{I}_{C}$ bestimmt aus $0 \rightarrow \mathrm{I}_{\max } ; \mathrm{O}: \mathrm{I}_{C}$ bestimmt aus $\mathrm{I}_{\max } \rightarrow-\mathrm{I}_{\max } ; \mathbf{X}: \mathrm{I}_{C}$ bestimmt aus $-I_{\max } \rightarrow I_{\max }$. a) Betrag der kritischen Stromstärke als Funktion des äußeren Feldes. b) Betrag der kritischen Stromstärke in der Reihenfolge der vorgenommenen Messungen. 
niederohmigen Zustand hingegen ist deutlich geändert. Der Widerstand ist stark von der Stromstärke abhängig und weist ein hohes Maximum um $I=0 \mu \mathrm{A}$ auf. Der maximale Widerstand im niederohmigen Zustand $(R \approx 100 \mathrm{k} \Omega)$ liegt dabei nur wenig unterhalb des maximalen Widerstands im hochohmigen Zustand $(R \approx 105 \mathrm{k} \Omega)$. Aufgrund der stärkeren Abhängigkeit des Widerstandes von der Stromstärke im niederohmigen Zustand lassen sich die Zustände dennoch klar trennen. Das geänderte Verhalten lässt sich entsprechend in den Strom-Spannungs-Kennlinien beobachten (Abb. 6.18b). Die Kennlinien beider Widerstandszustände weisen ein deutlich nichtlineares Verhalten auf.

Das Auftreten des Maximums im niederohmigen Zustand zeigt keinerlei Systematik, weder in Abhängigkeit des äußeren Feldes, noch in der Reihenfolge der vorgenommenen Messungen. Auch die Variation im Minimalwert des Widerstandes im niederohmigen Zustand (zwischen $7 \mathrm{k} \Omega$ und $30 \mathrm{k} \Omega$ ) weist keinerlei Abhängigkeit auf. Insgesamt ist der hochohmige Zustand deutlich besser definiert als der niederohmige.

Zur Analyse der kritischen Stromstärken, bei welchen sich der Widerstand abrupt ändert, werden auch die Daten des nicht abgebildeten Zweiges des Widerstandsverlaufes genutzt (s.o.). Es ergeben sich somit zwei Werte für die positive kritische Stromstärke. In Abbildung 6.19 a) sind die Beträge der kritischen Stromstärken in Abhängigkeit des äußeren Magnetfeldes dargestellt. Der Betrag der Stromstärke, bei dem das System vom nieder- in den hochohmigen Zustand wechselt, ist immer geringer als die Stromstärke für den gegensätzlichen Fall. Weiterhin liegt die schwarze Kurve fast ausschließlich über der roten Kurve. Nachdem die Probe also bei einem bestimmten Wert den niederohmigen Zustand und anschließend bei einem negativen Wert wieder den hochohmigen Zustand angenommen hat, ist eine höhere Stromstärke notwendig, um die Probe abermals in den niederohmigen Zustand zu bringen. Insgesamt zeigt sich aber eine deutliche Streuung aller Werte ohne systematische Abhängigkeit vom äußeren Feld. Auch für Messungen unter identischen Bedingungen streuen die kritischen Stromstärken: So ergibt sich beispielsweise die negative kritische Stromstärke für $H=20 \mathrm{kOe}$ einmal zu $I_{C}=-50 \mu \mathrm{A}$, bei einer weiteren Messung zu $I_{C}=-72 \mu \mathrm{A}$. Um eine mögliche Abhängigkeit von der Anzahl der bereits getätigten Schaltprozesse zu ermitteln, werden die kritischen Stromstärken in Abbildung 6.19b) in der Reihenfolge der vorgenommenen Messungen aufgetragen. Dabei ist $\mathrm{zu}$ beachten, dass die Zeit, welche zwischen einzelnen Messungen vergeht, stark variiert (von Minuten bis zu mehreren Stunden). Allenfalls für den zweiten Übergang in den niederohmigen Zustand kann eine leicht steigende Tendenz festgestellt werden. Eine Umrechnung der kritischen Stromstärken in kritische Stromdichten ergibt $J_{C} \approx 2 \cdot 10^{5} \mathrm{~A} / \mathrm{cm}^{2}$ für den Übergang vom hoch- in den niederohmigen Zustand, bzw. $J_{C} \approx 1 \cdot 10^{5} \mathrm{~A} / \mathrm{cm}^{2}$ für den gegensätzlichen Übergang.

In Abbildung 6.20 wird der Magnetowiderstand für eine Feldrichtung bei $T=5 \mathrm{~K}$ im hoch- (a), bzw. im niederohmigen Zustand (b) dargestellt. Für den hochohmigen Zustand (a) zeigt sich ein typisches Verhalten: Für kleine Felder dominiert der LFMR, während für hohe Felder der CMR überwiegt. Letzterer wird auch durch die zusätzlich aus den $R(I)$-Messungen gewonnenen Punkte deulich. Um eventuelle Interpretationsschwierigkeiten aufgrund des gelegentlich auftretenden Maximums im niederohmigen Zustand $\mathrm{zu}$ vermeiden, wird der Magnetowiderstand im niederohmigen Zustand mit $I=175 \mu \mathrm{A}$ 

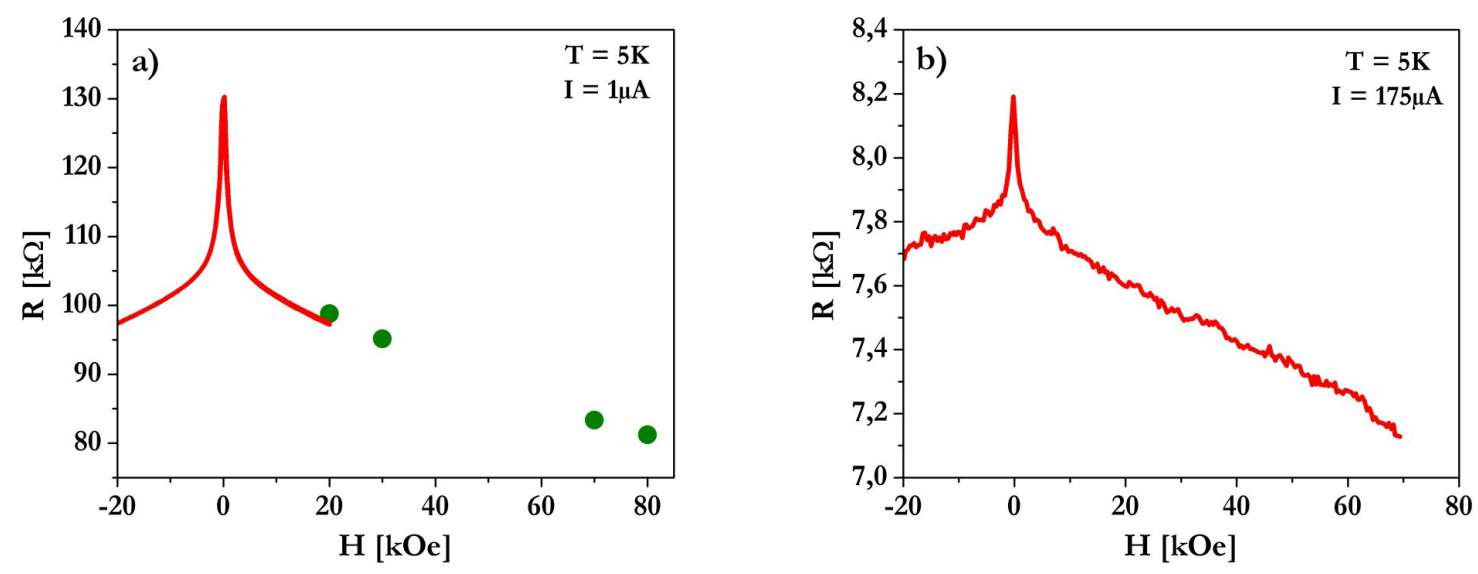

Abbildung 6.20. a) Magnetfeldabhängigkeit des elektrischen Widerstandes bei $\mathrm{T}=5 \mathrm{~K}$ im hochohmigen Zustand $(I=1 \mu \mathrm{A})$. Die zusätzlichen Punkte stammen aus R(I)-Messungen bei den jeweiligen magnetischen Feldern. b) Magnetfeldabhängigkeit des elektrischen Widerstandes bei $\mathrm{T}=5 \mathrm{~K}$ im niederohmigen Zustand $(I=175 \mu \mathrm{A})$.
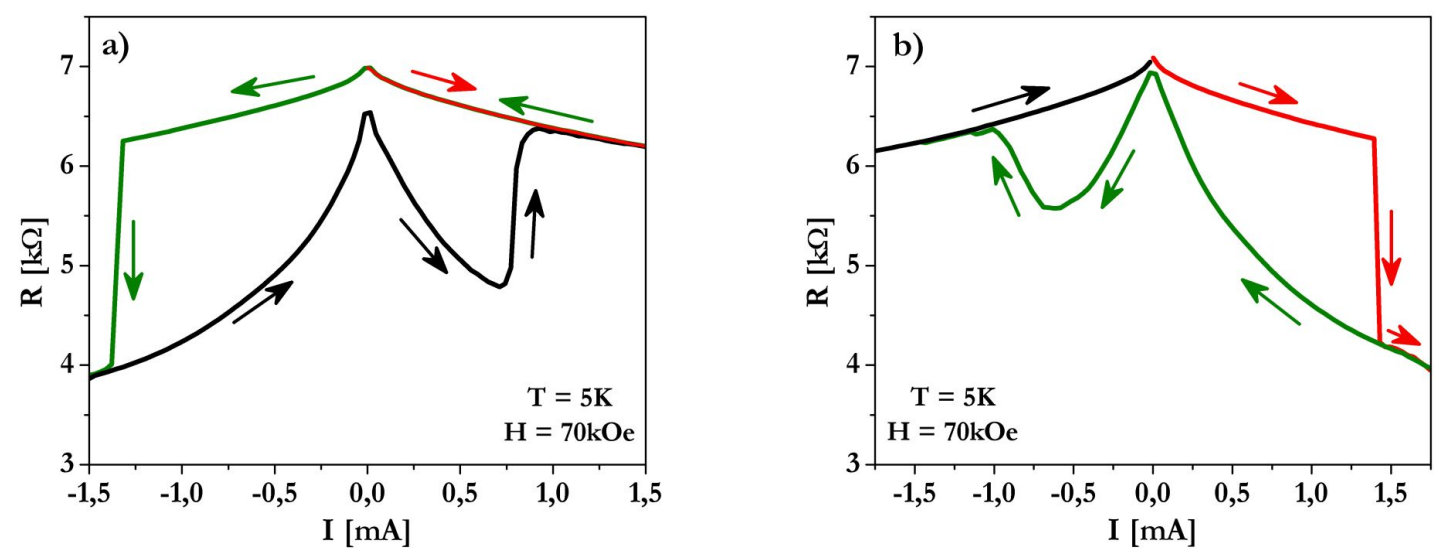

Abbildung 6.21. a) Widerstand des breiten Kontakts als Funktion der Stromstärke bei $T=5 \mathrm{~K}$ und $H=70 \mathrm{kOe}$. b) Messungen mit gewechselter Polarität bei sonst identischen Bedingungen.

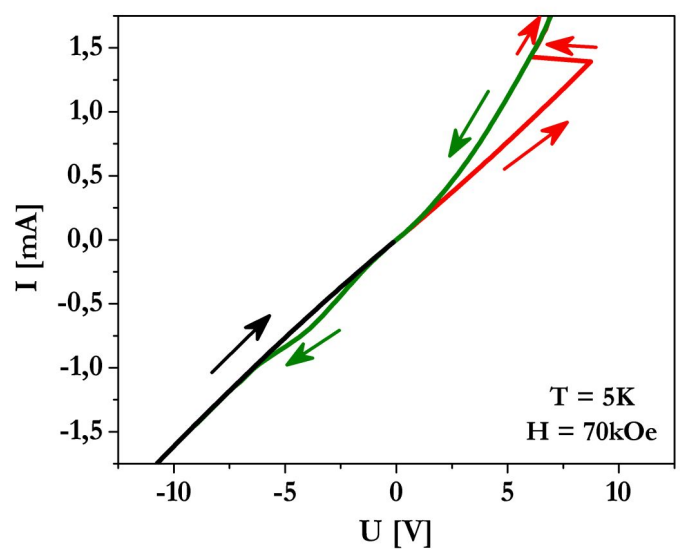

Abbildung 6.22. Strom-Spannungs-Kennlinie der Daten nach Abbildung 6.21 b). 
gemessen, also oberhalb der positiven kritischen Stromstärke. Es zeigt sich qualitativ das gleiche Verhalten wie in a): In kleinen Feldern ergibt sich eine starke Widerstandsänderung, für hohe Felder ist diese deutlich geringer und näherungsweise linear. Es ergibt sich ein LFMR von $5 \%$.

Weitere strominduzierte Messungen sind mit dem schmalen Kontakt nicht möglich, da der Widerstand im hochohmigen Zustand nach einer gewissen Anzahl von Messungen mit hohen Spannungen immer weiter ansteigt. Es ergeben sich keine weiteren reproduzierbaren Ergebnisse.

\section{Breiter Kontakt}

Um die abrupten, strominduzierten Widerstandsänderungen zu verifizieren, werden stromabhängige Widerstandsmessungen auch am breiten Kontakt durchgeführt. Abbildung 6.21 a) zeigt den stromabhängigen Widerstand des breiten Kontakts bei $T=5 \mathrm{~K}$ und $H=70 \mathrm{kOe}$ mit einer maximalen Stromstärke von $\left|I_{\max }\right|=1,5 \mathrm{~mA}$. Vor dieser Messung werden an diesem Kontakt nur stromabhängige Messungen mit $\left|I_{\max }\right| \leq 1,25 \mathrm{~mA}$ durchgeführt, in denen keine abrupten Widerstandsänderungen beobachtet werden. Dabei wird sowohl der positive, als auch der negative Maximalwert mit der üblichen Messroutine (s.o.) verwendet. Für $\left|I_{\max }\right|=1,5 \mathrm{~mA}$ jedoch zeigt sich das reversible Schalten zwischen hoch- und niederohmigen Zuständen. Dabei fällt auf, dass die erste abrupte Widerstandsänderung bei einer negativen Stromstärke von ca. $-1,3 \mathrm{~mA}$ auftritt, obwohl zuvor bereits ein positiver Strom gleicher Stärke durch die Probe geflossen ist: Die Widerstandsänderung ist an eine Richtung gekoppelt. Auffällig an den Messungen des breiten Kontakts ist der relativ geringe Widerstand von ca. $7 \mathrm{k} \Omega$ (hochohmiger Zustand) im Vergleich zum schmalen Kontakt. Zudem ist der kontinuierliche Abfall des Widerstandes im hochohmigen Zustand deutlich weniger ausgeprägt als für den schmalen Kontakt. Die relativen Widerstandsänderungen durch den Wechsel vom hoch- in den niederohmigen Zustand sind aber von vergleichbarer Größenordnung. Ferner tritt bei allen Messungen des breiten Kontaktes ein hohes Maximum um $I=0 \mathrm{~mA}$ auf, was für den schmalen Kontakt nur gelegentlich beobachtet wird.

Um die Abhängigkeit der Zustandsänderung von der Stromrichtung genauer zu untersuchen, wird die Polarität durch Wechseln der Anschlüsse an der Stromquelle untersucht. Die unter sonst identischen Bedingungen aufgenommene Messung zeigt Abbildung 6.21 b). Der Wechsel vom hoch- in den niederohmigen Zustand findet nun bei positiven Strömen statt, also bei gleicher Bewegungsrichtung der Elektronen in Bezug auf die Probe wie zuvor. Die zugehörige Strom-Spannungs-Kennlinie ist in Abbildung 6.22 dargestellt. Die Unterschiede in den Kennlinien der unterschiedlichen Widerstandszustände fallen nicht so deutlich aus wie in der $R(I)$-Auftragung. Der Übergang zwischen den beiden Widerstandszuständen tritt bei Spannungen auf, welche vergleichbar sind mit denen des schmalen Kontaktes.

Weitere strominduzierte Widerstandsänderungen werden bei einer Temperatur von $T=22 \mathrm{~K}$ beobachtet. Ohne äußeres Magnetfeld zeigt die Temperaturabhängigkeit des Widerstandes hier ein Minimum [s. Abb. 6.16a)]. Die kritischen Stromstärken sind ver- 
gleichbar mit denen bei $T=5 \mathrm{~K}$. Eine Berechnung der kritischen Stromdichten resultiert in $J_{C} \approx 1 \cdot 10^{6} \mathrm{~A} / \mathrm{cm}^{2}$ und liegt damit deutlich über denen des schmalen Kontaktes.

\section{Verifikation an Vergleichsprobe}

Stromabhängige Widerstandsmessungen werden auch an der $50 \mathrm{~nm}$ dicken LSMO-Probe durchgeführt, welche ebenfalls auf einem $\mathrm{Al}_{2} \mathrm{O}_{3}$-Substrat deponiert wird. Einerseits soll untersucht werden, ob die oben vorgestellten Effekte auch in anderen Proben gleicher Struktur auftreten, andererseits werden Experimente in Hinblick auf Symmetrieüberlegungen (s. Kap. 6.3.4 durchgeführt.

Auf der Schicht werden zwei Mikrostrukturen mit einer Breite von 1,0 $\mu \mathrm{m}$ und einer Länge von $1,0 \mu \mathrm{m}$ definiert. Die Messungen werden bei $T=5 \mathrm{~K}$ durchgeführt. Der temperaturabhängige Widerstand sowie der Kleinfeld-Magnetowiderstand, bestimmt mit einem Strom von $I=5 \mu \mathrm{A}$, zeigen ein qualitativ identisches Verhalten wie die $70 \mathrm{~nm}$ dicke Schicht.

Beide auf der Probe definierte Strukturen zeigen bei Anwendung des oben dargelegten Messprotokolls ein Widerstandsschalten, wie es für die vorherige Probe gefunden wird. Dabei ist festzuhalten, dass dieses Verhalten nicht in dem Maße reproduzierbar ist wie für die oben diskutierten Kontakte. So fällt beispielsweise die Widerstandsverminderung durch Erreichen des niederohmigen Zustandes nicht so deutlich aus wie für den schmalen Kontakt. Dennoch kann das Auftreten eines niederohmigen Zustandes für eine hohe Stromstärke bestimmter Polarität sowie die Rekonstruktion des Ausgangszustandes durch eine genügend hohe Stromstärke gegensätzlicher Polarität in beiden Kontakten dieser Probe verifiziert werden.

Weiterhin wird aufgrund der im Diskussionsteil getätigen Symmetrieüberlegungen bewusst die Stromstärke nach Erreichen des niederohmigen Zustands deutlich erhöht, was zu dem in Abbildung 6.23 dargestellten Ergebnis führt. Im Gegensatz zu den oben diskutierten Messungen (schmaler und breiter Kontakt) erreicht dadurch die abfallende Spannung, aufgrund der abrupten Widerstandsabnahme zunächst verringert, einen neuen Maximalwert [s. Abb. 6.23 b)]. Dies führt offenbar zu einem geänderten Widerstandsverhalten: Der Widerstand springt zurück in den hochohmigen Zustand. Bei Verringerung der Stromstärke sind zunächst keine abrupten Widerstandsänderungen zu beobachten. Für negative Stromstärken aber zeigt sich das identische Verhalten. Nach Erreichen des niederohmigen Zustandes wird der hochohmige Zustand wieder hergestellt. Es ergibt sich insgesamt ein symmetrisches Verhalten.

\section{Epitaktische Vergleichsprobe}

Zur Evaluation des Einflusses der Korngrenzen auf das Widerstandsschalten werden die elektronischen Eigenschaften einer epitaktischen LSMO-Probe charakterisiert, welche auf einem (001)-orientierten MgO-Substrat präpariert wird. Dabei ist insbesondere das strombzw. spannungsabhängige Widerstandsverhalten von Interesse. Um möglicherweise auftretende Effekte mit den Ergebnissen der nanokristallinen Probe vergleichen zu können, wird 

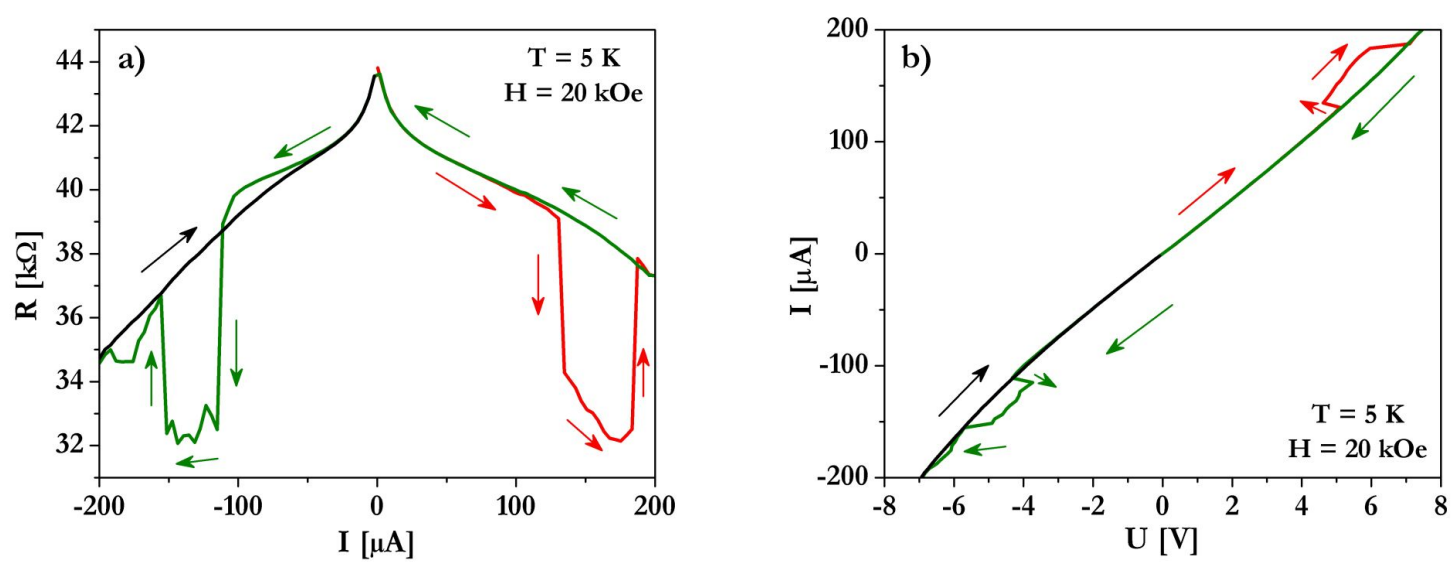

Abbildung 6.23. Zur Untersuchung der Symmetrie durchgeführte Untersuchung eines weiteren Kontaktes. a) Widerstand als Funktion der Stromstärke bei $T=5 \mathrm{~K}$ und $H=20 \mathrm{kOe}$. b) Strom-Spannungs-Kennlinie der Daten nach a).

eine nahezu identische Mikrostruktur auf der $50 \mathrm{~nm}$ dicken Schicht definiert. Die Stegbreite beträgt ca. $2 \mu \mathrm{m}$, der Abstand der beiden Goldelektroden 1,5 $\mu \mathrm{m}$.

Der elektrische Widerstand dieser Struktur beträgt ca. $400 \Omega$ bei $T=3,5 \mathrm{~K}$, was einem spezifischen Widerstand von etwa $3 \cdot 10^{-3} \Omega \cdot \mathrm{cm}$ entspricht. Die Magnetfeldabhängigkeit des Widerstandes ist bei tiefen Temperaturen sehr gering, wie der in Abbildung 6.24 a) dargestellte Magnetowiderstand bei $T=3,5 \mathrm{~K}$ zeigt. Es ergibt sich bis zu Feldern von $70 \mathrm{kOe}$ eine nahezu lineare Abnahme des Widerstandes, wobei die maximale Änderung nur 1,3\% Prozent beträgt. Ein positiver Kleinfeld-Magnetowiderstand ist nicht zu beobachten.

Der Widerstand als Funktion der Stromstärke ist in Abbildung 6.24b) dargestellt. Es findet die Messvorschrift Verwendung, welche auch für die nanokristallinen Proben eingesetzt wird. Die maximale Stromstärke der aufeinander folgenden Messreihen wird dabei sukzessive erhöht. Die erste dargestellte Messreihe, welche die oben definierte Farbkodierung verwendet, hat eine maximale Stromstärke von $\left|I_{\max }\right|=10 \mathrm{~mA}$. Es zeigt sich ein von der nanokristallinen Probe grundsätzlich verschiedenes Verhalten. Mit zunehmendem Betrag der Stromstärke steigt der Widerstand quadratisch an. Dabei ergibt sich ein nahezu reversibles Verhalten ohne Hysterese. Lediglich für positive Stromstärken ist ein geringer Unterschied zwischen dem Widerstand für zu- und dem für abnehmende Stromstärke zu beobachten, welcher jedoch einerseits klein ist und andererseits auf leichte Temperaturschwankungen zurückgeführt werden kann. Auf der oberen Abszisse ist die am Kontakt abfallende Spannung aufgetragen. Diese Auftragung gilt strenggenommen nur für die grüne Kurve und ist aufgrund des sich ändernden Widerstandes schwach nichtlinear. Der Maximalstrom von $I_{\max }=10 \mathrm{~mA}$ entspricht einer maximal abfallenden Spannung von $4,6 \mathrm{~V}$.

Eine weitere Erhöhung der maximalen Stromstärke auf $\left|I_{\max }\right|=15 \mathrm{~mA}$ hat die Zerstörung des Kontaktes zur Folge. Zunächst folgt der Widerstand für $I \leq 10 \mathrm{~mA}$ im Wesentlichen dem quadratischen Verlauf, wie er zuvor für die Messung mit geringerer maximaler Stromstärke beobachtet wird (Kurve 4 in Abbildung 6.24 b). Nach Überschreiten von $I=10,5 \mathrm{~mA}$, was einer abfallenden Spannung von $U=4,9 \mathrm{~V}$ entspricht, wird der Wider- 

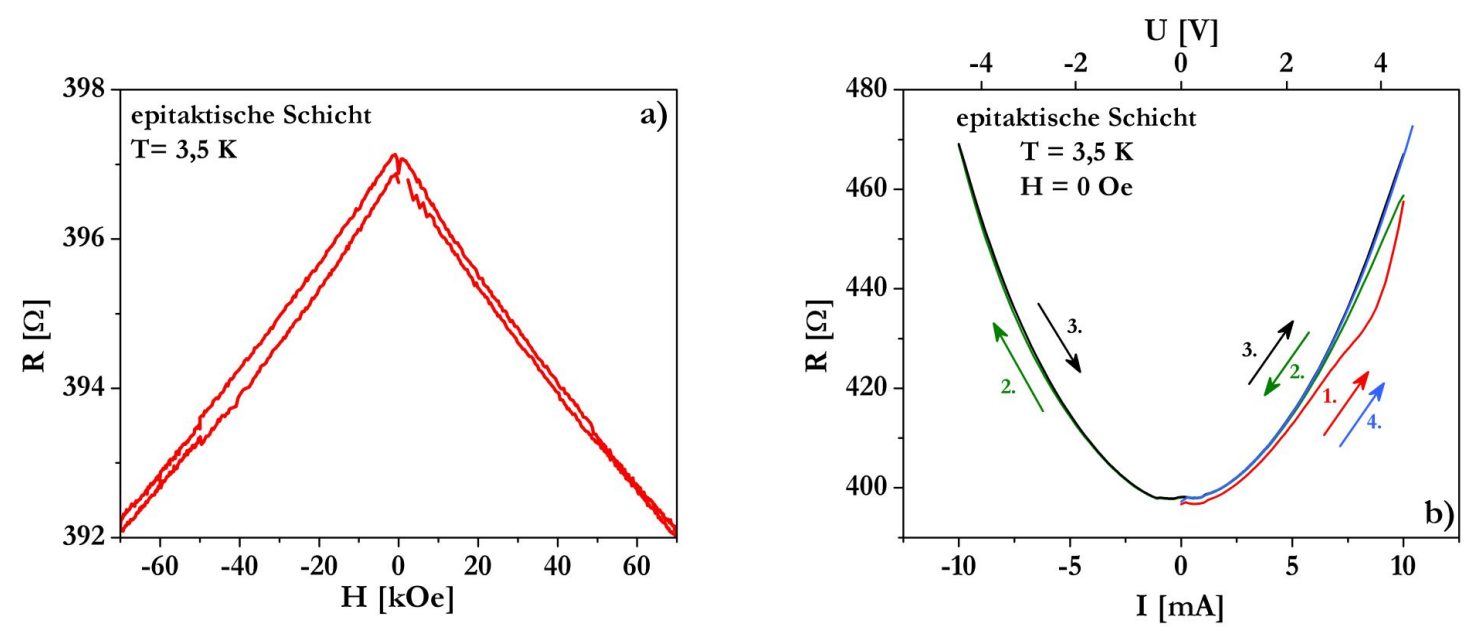

Abbildung 6.24. Elektronische Eigenschaften der epitaktischen Vergleichsprobe bei $T=$ $3,5 \mathrm{~K}$. a) Magnetowiderstand mit $I=5 \mu \mathrm{A}$. b) Widerstand als Funktion des Stromes ohne äußeres Feld. Die auf der oberen Abszisse aufgetragenen Spannungswerte gelten strenggenommen nur für die grüne Kurve und ist nichtlinear.

stand unmessbar großs. Dies ändert sich auch nach einer langen Wartezeit (ein Tag) nicht und gilt für beide Polaritäten.

\subsubsection{Diskussion}

\section{Struktur und Magnetotransport}

Die Röntgenstrukturanalyse der $70 \mathrm{~nm}$ dicken, auf $\mathrm{Al}_{2} \mathrm{O}_{3}$ deponierten Schicht lässt auf polykristallines Wachstum von LSMO mit einer dominanten (111)-Orientierung schließen. Der sich ergebende Gitterparameter von $a=3,86 \AA$ liegt nur wenig unterhalb dem Wert für Volumenproben [59], die Probe ist also weitgehend unverspannt. Die Größe der in (111)-Richtung kohärent streuenden Bereiche ergibt sich aus der Scherrer-Analyse zu $\tau \approx 45 \mathrm{~nm}$. Dies ist vergleichbar mit der Schichtdicke von $70 \mathrm{~nm}$, wobei in Betracht gezogen werden muss, dass die Scherrer-Analyse lediglich eine mittlere Größe der kohärent streuenden Bereiche liefert. Weiterhin kann der Unterschied zwischen Schichtdicke und dem Ergebnis der Scherrer-Analyse durch Oberflächenrauigkeiten der Schicht und Grenzflächeneinflüsse der einzelnen Körner erklärt werden. So kann geschlossen werden, dass zumindest ein nennenswerter Anteil der Körner ein kolumnares Wachstum aufweist, also vom Substrat bis zur Schichtoberfläche verläuft. Die mittlere laterale Korngröße ergibt sich aus der REM-Analyse der Oberfläche zu ungefähr $50 \mathrm{~nm}$.

Sowohl die unverspannte Struktur als auch der polykristalline Charakter manifestieren sich in den Ergebnissen des Magnetotransports, welche typisch für polykristallines LSMO sind [31]. Die unverspannte Struktur ist eng verknüpft mit nahezu idealen Phasenübergangstemperaturen. Diese bestätigen wiederum die Stöchiometrie, da die Übergangstemperaturen bei Änderung der Zusammensetzung tiefer lägen. Das Verhältnis von $R(T=5 K)$ zu $R\left(T_{M I}\right)$ beträgt ca. $1 / 6$ und ist somit deutlich höher als für epitaktisch gewachsene Filme oder Einkristalle [12]. Hierin spiegelt sich der Einfluss der Korngrenzen 
wider, welche ausgedehnte, statische Defekte für die Leitungselektronen darstellen und so den Restwiderstand erhöhen [46]. Weiterhin können sie über eine Coulomb-Blockade das beobachtete Minimum im temperaturabhängigen Widerstandsverlauf erklären [30]. Die Korngrenzen sind auch für den auftretenden LFMR verantwortlich [27], wobei die genaue Struktur der Korngrenze Gegenstand aktueller Diskussionen ist, siehe Kapitel 3.4. Die Korngrenzen haben also einen wesentlichen Einfluss auf die Transporteigenschaften der Probe.

Die Mikrostrukturierung der Probe führt zu keiner qualitativen Veränderung der Transporteigenschaften. Allerdings zeigt sich eine deutliche Erhöhung des absoluten Widerstandswertes, welche nicht nur durch die geänderte Geometrie erklärt werden kann. Einerseits können Inhomogenitäten wie die in den REM-Bildern aufgezeigten Größenunterschiede der Körner dafür verantwortlich sein, andererseits können auch eventuelle Kontaktwiderstände nicht ausgeschlossen werden, da keine echte Vier-Punkt-Messung vorgenommen wird. Weiterhin ist auffallend, dass der Widerstand nicht mit der Breite der Mikrostruktur skaliert. Für die breite Struktur liegt der Widerstand im hochohmigen Zustand in der Größenordnung von $7 \mathrm{k} \Omega$, für die um einen Faktor $\approx 2,5$ schmalere Struktur (bei sonst identischer Geometrie) bei $100 \mathrm{k} \Omega$. Neben Inhomogenitäten kann auch ein erhöhter Einfluss der Randbereiche für diesen Anstieg verantwortlich sein.

\section{Strominduziertes Widerstandsschalten}

Zur Diskussion der strominduzierten Effekte werden die $R(I)$-Kurven in drei Bereiche aufgeteilt. Prozess I stellt den kontinuierlichen Abfall des Widerstands im hochohmigen Zustand dar. Bereich II betrifft die abrupten Widerstandsänderungen zwischen den beiden Zuständen und Prozess III beschreibt das gelegentliche Auftreten des hohen Maximums im niederohmigen Zustand. Dabei folgt aus dieser Unterteilung keine Unabhängigkeit der einzelnen Prozesse, sie soll zunächst lediglich die Diskussion vereinfachen.

\section{Bereich I}

Der kontinuierliche Abfall des Widerstandes im hochohmigen Zustand tritt unabhängig von Prozess II auf: Er ist auch zu beobachten, wenn die $R(I)$-Kurven mit $I_{\max }<I_{\mathrm{C}}$ aufgenommen werden. In diesen Fällen wird keine Hysterese im Widerstandsverlauf beobachtet. Die Verringerung des Widerstands mit zunehmender Spannung, also das Auftreten eines nichtohmschen Verhaltens, lässt sich mit einem Tunneln der Leitungselektronen über die Korngrenzen hinweg deuten. Die Annahme der Existenz solcher Tunnelbarrieren wird durch den beobachteten LFMR erhärtet. Abbildung 6.25 zeigt repräsentativ die Leitfähigkeit $G(U)=d I / d U$ im hochohmigen Zustand für den schmalen Kontakt bei $H=5 \mathrm{kOe}$ und $T=5 \mathrm{~K}$ (vgl. Abb. 6.17). Eine Beschreibung der Daten durch das Modell von Simmons (s. Kap. 3.1.3 gelingt gut, abgesehen von einem Bereich kleiner Spannungen. Dabei sind die hier beobachteten Abweichungen vom parabolischen Verhalten im Bereich kleiner Spannungen typisch für (magnetische) Tunnelkontakte und können vielfältige Ursachen haben. In magnetischen Tunnelkontakten spielt dabei das magnonenunterstützte, inelastische Tunneln eine wichtige Rolle [116, 198, aber auch andere Prozesse wie loka- 


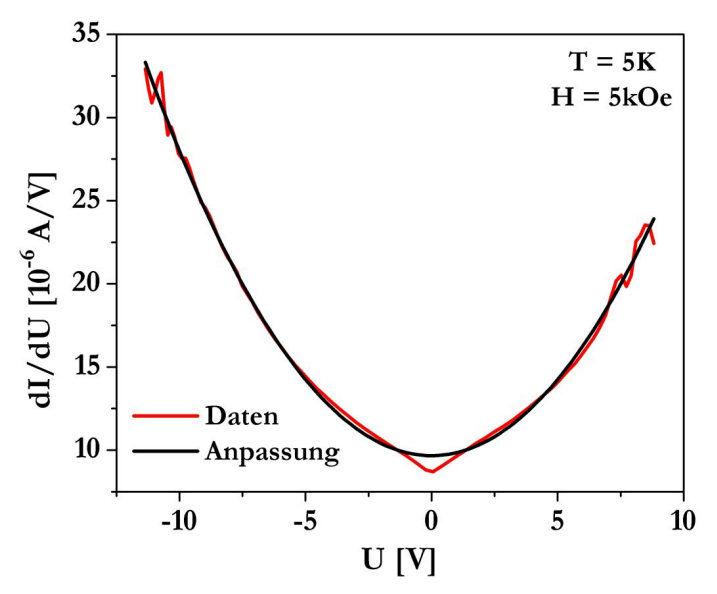

Abbildung 6.25. Leitfähigkeit dI/dU der Daten nach Abbildung 6.17 im hochohmigen Widerstandszustand sowie Anpassung nach Simmons (Formel 3.7).

lisierte, paramagnetische Verunreinigungen an der Grenzfläche zum Isolator |199| oder Mehr-Stufen-Tunneln können diese so genannte zero bias anomaly erklären [117].

Die Beschreibung der Leitfähigkeit im hochohmigen Zustand gelingt zwar durch das Simmons-Modell, allerdings erscheinen die extrahierten Parameter von $\phi_{0} \approx 57 \mathrm{eV}$ und $d \approx 0,5 \mathrm{~nm}$ unphysikalisch. Hierbei ist zu beachten, dass das Simmons-Modell den Transport durch einen einzelnen, planaren Tunnelkontakt beschreibt. Diese Voraussetzung ist nicht gegeben. Vielmehr muss ein Netzwerk von ca. $20 \times 30$ Tunnel-Elementen berücksichtigt werden. Unter der stark vereinfachten Annahme einer Reihenschaltung von 30 identischen, planaren Tunnelkontakten fällt jeweils nur 1/30 der Spannung an einem Kontakt ab. Eine solche Modifikation der Simmons-Formel führt zu einem identischen Ergebnis in der Anpassung der Leitfähigkeit, die extrahierten Parameter erscheinen nun aber sinnvoll: $\phi_{0} \approx 1,8 \mathrm{eV}$ und $d \approx 3 \mathrm{~nm}$. Für eine genaue quantitative Analyse ist eine detaillierte Betrachtung des Netzwerks der Tunnelelemente nötig.

Die kontinuierliche Abnahme des Widerstandes im hochohmigen Zustand kann folglich gut durch einen Tunneleffekt beschrieben werden. Allerdings schließt dies andere Effekte als dominante Ursache für die Widerstandsabnahme nicht aus. So ist beispielsweise eine Verknüpfung mit Prozess II denkbar. Ohne Prozess II zunächst mikroskopisch zu beschreiben, ist ein Szenario denkbar, in dem sich eine zweite Phase größerer Leitfähigkeit bildet, welche bei $I=I_{C}$ einen leitfähigen Pfad durch die Probe formt. Ein Anwachsen des Volumenanteils dieser Phase für $I<I_{C}$ hätte eine kontinuierliche Widerstandsverringerung zur Folge. Eine quantitative Analyse benötigt die genaue Kenntnis der Verteilung dieser Volumenanteile, welche aber nicht erfasst werden kann, s.d. eine Verifizierung bzw. Negierung dieses Szenarios nicht möglich ist. Wird jedoch stark vereinfacht angenommen, dass im tiefsten Widerstandszustand 100\% des Volumens im leitfähigen Zustand sind, so können insbesondere die Widerstandsvariationen des niederohmigen Zustand nur durch extreme Variationen in der Pfadbreite erklären werden. Insgesamt erscheint eine Erklärung der kontinuierlichen Widerstandsabnahme aufgrund eines Tunneleffekts wahrscheinlicher. Im Folgenden wird deshalb Prozess I als unabhängig von Prozess II 
angesehen.

\section{Bereich II}

Im Gegensatz zu Prozess I stellt Prozess II eine reversible, aber hysteretische Widerstandsänderung dar. Nach Überschreiten einer kritischen Stromstärke existieren im Bereich $I_{C}^{\text {neg }}<I<I_{C}^{\text {pos }}$ zwei unterschiedliche Widerstandszustände, welche durch Überschreiten einer kritischen Stromstärke definierter Polarität ineinander überführt werden können. Dabei stellt der niederohmige Zustand einen neuen, durch Variation anderer äußerer Parameter wie Temperatur und Magnetfeld nicht erreichbaren Zustand dar. So tritt beispielsweise für die breite Struktur auch für $T=22 \mathrm{~K}$ in einem Feld von $H=70 \mathrm{kOe}$ das Widerstandsschalten auf. Der hochohmige Zustand liegt dabei in der Nähe eines Minimums im T-H-Raum. Aus dieser Tatsache kann eine thermische Ursache für das Widerstandsschalten ausgeschlossen werden: jede Temperaturänderung führt zu einer Widerstandserhöhung. Im Falle thermischer Mechanismen müssten weiterhin alle Effekte unabhängig von der Stromrichtung sein, was nicht der Fall ist.

Auch das strominduzierte Magnetisierungsschalten (s. Kap. 3.3) kann als Ursache für die Widerstandsänderungen weitgehend ausgeschlossen werden. Zwar könnte dieser Mechanismus die Asymmetrie in den $R(I)$-Kurven erklären, allerdings sollte sich eine deutliche Magnetfeldabhängigkeit in den kritischen Strömen zeigen [122]. Weiterhin favorisiert ein hohes, von außen angelegtes Magnetfeld die parallele Orientierung der magnetischen Momente. Eine durch eine hohe Stromdichte hervorgerufene Abweichung von dieser Konfiguration würde demnach eine Widerstandserhöhung nach sich ziehen. Das Gegenteil wird beobachtet. Zudem zeigen die berechneten kritischen Stromdichten für die breite und schmale Struktur große Abweichungen voneinander, wobei insbesondere der Absolutwert für die schmale Struktur von $J_{C} \approx 2 \cdot 10^{5} \mathrm{~A} / \mathrm{cm}^{2}$ zu klein für ein Magnetisierungsschalten erscheint 109,7). Auch die Beobachtung des LFMR im niederohmigen Zustand, also das Auftreten unterschiedlicher Magnetisierungskonfigurationen, spricht gegen das strominduzierte Magnetisierungsschalten.

Ein Vergleich der kritischen Stromstärken, bzw. Stromdichten der breiten und der schmalen Struktur lässt Zweifel an der Interpretation aufkommen, dass der Strom verantwortlich für das Widerstandsschalten ist. Sie liegen ca. eine Größenordnung auseinander. Vielmehr zeigt sich, dass die jeweiligen Spannungen, bzw. elektrischen Felder in der Nähe der Schaltprozesse vergleichbar sind (ca. $U \approx 5-10 \mathrm{~V}$, bzw. $E \approx 4-7 \cdot 10^{4} \mathrm{~V} / \mathrm{cm}$ ). Somit erscheint es wahrscheinlicher, dass das elektrische Feld entscheidend für das Widerstandsschalten ist. Ein Einfluss des Stromes kann zwar nicht ausgeschlossen werden, im Weiteren soll jedoch das elektrische Feld als verantwortlich bezeichnet werden.

Die Charakteristika der Messungen weisen große Ähnlichkeiten mit EPIR-Experimenten (s. Kap. 2.6) auf. Zwar werden die Untersuchungen in dieser Arbeit nicht mit gepulsten Spannungen durchgeführt, dennoch sind massive Widerstandsänderungen nach Überschreiten einer gewissen Spannung mit definierter Polarität zu beobachten. Das Überschreiten einer kritischen Spannung bei geänderter Polarität stellt dabei reversibel den Ausgangszustand wieder her. Die beobachteten kritischen elektrischen Felder liegen zwar etwas über den üblicherweise bestimmten Werten, sind aber in der Größenordnung 
vergleichbar 87.

Eine Erklärung der Widerstandsänderungen mittels Mechanismen, welche den EPIR modellieren, ist in rein metallischen System nicht möglich (s. Kap. 2.6). Hierbei ist anzumerken, dass die beiden Mikrostrukturen, an denen das Widerstandsschalten weit unterhalb der Phasenübergangstemperatur beobachtet wird, einen sehr hohen absoluten Widerstand aufweisen. Zum einem stellt eine mögliche Grenzschicht zur Elektrode einen hohen Kontaktwiderstand dar, andererseits ist auch die hohe Anzahl der Korngrenzen zu beachten, welche den Transportprozess maßgeblich beeinflusst. Die Korngrenzen weisen dabei kein metallisches Verhalten auf (s. Kap. 3.4).

\section{Einfluss der Korngrenzen}

Die Untersuchungen des strom- bzw. spannungsabhängigen Widerstandes der epitaktischen Vergleichsprobe mit zu den Nanokristallen identischen Mikrostrukturen weisen auf die Bedeutung der Korngrenzen für das Widerstandsschalten hin. So tritt in dieser Arbeit das für den EPIR charakteristische, stabile, polaritätsabhängige Schalten zwischen einem hochohmigen und einem niederohmigen Zustand nur in den nanokristallinen Schichten auf. In der epitaktischen Vergleichsprobe zeigt sich dagegen ein vollkommen geändertes Verhalten (s. Abb. 6.24): Aufgrund von Heizeffekten kommt es zu einer quadratischen Zunahme des Widerstandes, welcher aufgrund der Struktur deutlich geringer ist als bei den nanokristallinen Proben. Das Widerstandsverhalten ist reversibel und nichthysteretisch. Abrupte Widerstandsänderungen werden vor Zerstörung des Kontaktes durch die hohe Stromstärke nicht beobachtet. Hieraus können zwei Schlüsse gezogen werden: Einerseits kann für den vorliegenden Fall ein Einfluss des Kontaktwiderstandes, bzw. einer eventuell vorhandenen, dünnen Grenzschicht des Manganats zur Elektrode, welche in manchen Publikationen als Ursache für das hysteretische Verhalten angesehen wird [85, 200], ausgeschlossen werden. Eine solche Grenzschicht sollte auch für die epitaktische Probe vorhanden sein, worauf auch der gegenüber dem Volumenwert erhöhte spezifische Widerstand deutet. Dennoch sind keine Widerstandseffekte wie für die nanokristallinen Schichten zu beobachten. Weiterhin kann geschlossen werden, dass die Korngrenzen essentiell für das Widerstandsschalten sind und eine wesentliche Voraussetzung für den Schaltprozess darstellen. Dabei ist zu beachten, dass die kritischen Feldstärken, welche für die nanokristallinen Schichten ermittelt werden, nicht erreicht werden. Entsprechend kann zunächst keine Aussage getroffen werden, ob die Korngrenzen ursächlich für die Widerstandsänderungen sind, oder ob sie eventuell eine notwendige Voraussetzung zur Erhöhung der Feldstärke darstellen.

Xie et al. zeigen ein stabiles Widerstandsschalten bei Raumtemperatur in LSMOSchichten, welche aber mit einer stark oszillierenden Substrattemperatur $\left(400^{\circ} \mathrm{C}-1000^{\circ} \mathrm{C}\right)$ deponiert werden [87]. Die Autoren schließen daraus sofort auf eine hochohmige Grenzschicht zur Elektrode, welche notwendig zur Beobachtung des Widerstandsschaltens sei. In Schichten mit hoher Oberflächengüte beobachten sie keinen Effekt [87]. Dabei ist anzumerken, dass bei solch starken Temperaturschwankungen während der Deposition die genaue Mikrostruktur unklar bleibt. Dennoch kann davon ausgegangen werden, dass die Schichten inhomogen sind, was auf die Relevanz von strukturellen Defekten für das Wi- 
derstandsschalten in LSMO deutet.

Diese Annahme ist konsistent mit weiteren Modellen des EPIR [92, 93, 94, 88, in denen Inhomogenitäten und ausgedehnte Defekte als wesentlich für den EPIR angesehen werden. So zeigen Szot et al., dass in sauerstoffdefizitärem $\mathrm{SrTiO}_{3}$ der lokal modulierte Sauerstoffgehalt in Versetzungen verantwortlich für das stabile Widerstandsschalten ist 94. Auch Nian et al. führen den EPIR mittels temperaturabhängiger Untersuchungen auf eine Sauerstoffdiffusion in einem Bereich nahe der Elektroden zurück [88]. Dabei ist zu beachten, dass Korngrenzendiffusion allgemein eine niedrigere Aktivierungsenergie aufweist als Volumendiffusion [174], was abermals auf die Bedeutung von Inhomogenitäten hinweist.

Für die in dieser Arbeit untersuchten nanokristallinen Schichten erscheint eine Beeinflussung des Korninneren unwahrscheinlich, da hier der metallische Grundzustand vorliegt, und mögliche Mechanismen demnach versagen. Zudem ist zu beachten, dass der Effekt vermutlich durch das elektrische Feld verursacht wird. In den nanokristallinen Systemen fällt die Spannung aber im Wesentlichen an den Korngrenzen ab. Weiterhin deutet der Vergleich mit dem epitaktischen System auf eine Beteiligung der Korngrenzen. Im Folgenden soll deshalb eine Modifikation der Korngrenzen als ursächlich für die Widerstandsänderungen angesehen werden.

Ein denkbarer Mechanismus der Modifikation der Korngrenzen ist die Beeinflussung ihres lokalen Sauerstoffgehalts 88, 94. Dabei werden alle Experimente, in denen dieser Zusammenhang vermutet wird, nahe Raumtemperatur durchgeführt. Eine Sauerstoffdiffusion bei $T=5 \mathrm{~K}$ erscheint jedoch wenig wahrscheinlich. So werden beispielsweise wesentliche Einflüsse von Sauerstoff-Diffusion auf den Widerstand von $\mathrm{La}_{0,8} \mathrm{Sr}_{0,2} \mathrm{MnO}_{3^{-}}$ Pulverproben erst bei $T=700^{\circ} \mathrm{C}$ gefunden 201. Vielmehr ist ein Mechanismus ähnlich zum Ordnungs-Unordnungs-Übergang vorstellbar, wie er von Jooss et al. in PCMO beobachtet wird 22. Dabei wird dieser Übergang vermutlich durch eine Rotation des $\mathrm{MnO}_{6}$-Oktaeders verursacht [22], welche bei tiefen Temperaturen wahrscheinlicher ist als Sauerstoffdiffusion. Weiterhin stellen die Autoren fest, dass sich die neu entstehende Phase zumeist an Defekten bildet, also eine Art von heterogener Nukleation stattfindet, was wiederum auf die Bedeutung der Korngrenzen deutet. Im vorliegenden Fall könnte ein solcher Mechanismus zur Verringerung der effektiven Barrierenbreite führen. Dies hätte zur Folge, dass zum Aufbau eines kritischen elektrischen Feldes über eine Korngrenze hinweg im niederohmigen Zustand eine geringere Spannung notwendig wäre als im hochohmigen Zustand. Finden die Widerstandsänderungen aber bei gleichen elektrischen Feldern statt, so entspräche das einer geringeren kritischen Spannung im niederohmigen Zustand, wie tatsächlich beobachtet wird. Ein mögliches mikroskopisches Modell der Änderung der Korngrenzeneigenschaften wird unten gegeben.

In mehreren Experimenten wird schließlich die Veränderung des Materials nur in der Nähe der Elektroden beobachtet $85,86,87,89,90,200,202$. Die angegebenen räumlichen Ausdehnungen liegen dabei zwischen $10 \mathrm{~nm}$ |85| und einigen $\mu \mathrm{m}$ 202. Übertragen auf die Geometrie der hier untersuchten Kontakte können die betroffenen Bereiche einerseits sehr lokal begrenzt sein, s.d. der Großteil der Korngrenzen unbeeinflusst bleibt, andererseits liegen die von Chen et al. 202 gewonnenen Abmessungen im Bereich der Länge des 
Kontaktes, bzw. darüber.

Unter der Annahme, dass der Effekt auf einen Bereich nahe der Elektroden beschränkt ist und nicht den gesamten Kontakt betrifft, ließen sich weitere Charakteristika der Messungen wie Bereich III und die Schwankungen des Widerstandes des niederohmigen Zustandes für unterschiedliche Messungen erklären. Ist beispielsweise die Größe des transformierten Bereichs in einem kleinen elektrischen Feld $\left(I<<I_{C}\right)$ zeitabhängig, so ist ein kontinuierliches Ansteigen des Widerstandes zu beobachten. Zur Erklärung des beobachteten Bereichs III wäre ein Relaxationsprozess mit einer Zeitkonstante von der Größenordnung der Messdauer, also im Bereich von Sekunden bis Minuten, notwendig. Experimente, in denen das zeitabhängige Widerstandsverhalten im niederohmigen Zustand bei kleinen Stromstärken untersucht werden sollte, waren jedoch nicht erfolgreich. Im Rahmen dieser Messungen trat immer ein breites Maximum im Widerstandsverhalten des niederohmigen Zustands für kleine Stromstärken auf, so dass die saubere Präparation eines niederohmigen Zustands für kleine Stromstärken nicht zu realisieren war. Gegen eine Zeitkonstante der Größenordnung einiger Sekunden spricht die in der Literatur berichtete Stabilität der Widerstandszustände von teilweise über 18 Monaten [83|. Neben Bereich III könnten auch die Variationen des Widerstandes im niederohmigen Zustand erklärt werden. Sie ergeben sich möglicherweise aus unterschiedlichen Volumenanteilen der transformierten Bereiche.

Andererseits können die aufgeführten Effekte auch durch andere Mechanismen erklärt werden. So ergeben sich die Widerstandsvariationen des niederohmigen Zustandes auch aus Konsequenzen von Symmetrieüberlegungen, welche im folgenden Abschnitt dargestellt werden. Eine weitere Erklärung für das Verhalten in Bereich III folgt dagegen aus einem mikroskopischen Modell, welches anschließend erarbeitet wird. Letztlich kann daher keine Aussage über die laterale Ausdehnung des betroffenen Bereiches getroffen werden.

\section{Symmetrieüberlegungen}

Obwohl eine Erklärung der strominduzierten Widerstandsänderungen mit dem oben entworfenen Bild, also einem EPIR-Mechanismus, der auf die Korngrenzen beschränkt ist, gut gelingt, bleibt das generelle Problem des asymmetrischen Widerstandsverhaltens in einem hochsymmetrischen Kontakt zunächst ungeklärt. In den meisten EPIR-Experimenten kommen verschiedene Elektrodenmaterialien oder auch unterschiedlich große Kontaktflächen zum Einsatz, so dass eine Asymmetrie, welche das polare Widerstandsverhalten erklären kann, im Kontakt als solchen bedingt ist. In einem strukturell symmetrischen Kontakt, wie den hier untersuchten, wirft dieses Verhalten hingegen die Frage nach der Verletzung der Paritäts-Erhaltung in elektromagnetischen Feldern auf [200|. Eine mögliche Lösung liegt in Inhomogenitäten der Probe oder Trainingseffekten 200. Chen et al. zeigen jedoch, dass ein Widerstandsschalten, basierend auf dem EPIR, auch in hochsymmetrischen Kontakten ohne Verletzung der Paritätserhaltung auftreten kann [203]. Die von ihnen durchgeführten Experimente und Simulationen können gegebenenfalls das hier beobachtete Verhalten erklären.

Chen et al. erhalten unter anderem die in Abbildung 6.26 dargestellten Ergebnisse für spiegelsymmetrische PCMO-Kontakte. Dargestellt ist der Widerstand des gesamten Kon- 

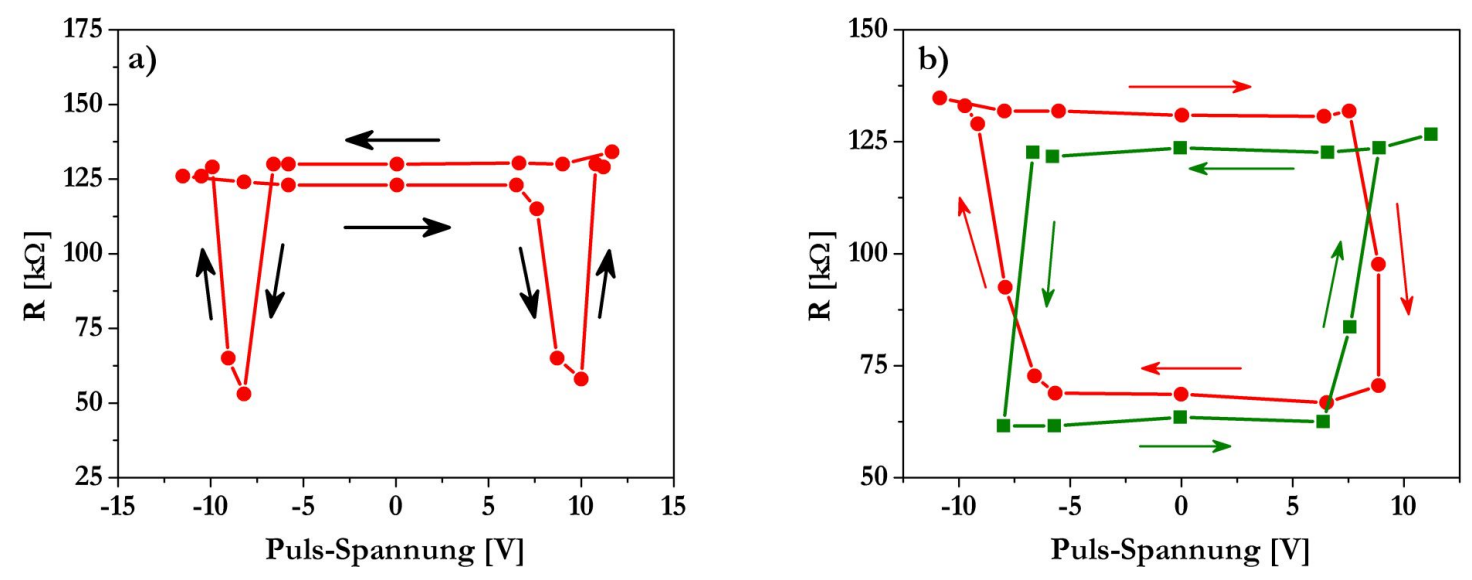

\begin{abstract}
Abbildung 6.26. Ergebnisse von Chen et al:: a) Widerstandsverhalten eines spiegelsymmetrischen PCMO-Kontakts bei Raumtemperatur. b) Widerstandsverhalten des identischen Kontakts bei Einschränkung der Spannungpulse auf -9 bis $12 \mathrm{~V}$ (rote Kreise), bzw -12 bis $9 \mathrm{~V}$ (grüne Quadrate). Nach 203.
\end{abstract}

takts, bestimmt durch einen Strom von $1 \mu \mathrm{A}$ nach Anlegen des auf der Abszissenachse aufgetragenen Spannungspulses. In Abbildung 6.26 a) sind in einem Bereich $7 \mathrm{~V} \leq|U| \leq 11 \mathrm{~V}$ massive Widerstandsänderungen zu beobachten. Für $|U|>11 \mathrm{~V}$ geht der Widerstand auf seinen Ausgangswert zurück. Werden die maximalen Spannungspulse aber so geändert, dass beispielsweise der maximale negative Spannungspuls das System in den niederohmigen Zustand bringt, so bleibt dieser Zustand stabil und ein Spannungspuls von $U>7 \mathrm{~V}$ ist nötig, um den hochohmigen Zustand wieder zu erlangen. Diese Art von Messung (in magnetischen Lagensystemen auch ,minor-loops“ genannt) ist in Abbildung 6.26 b) dargestellt und kann entsprechend auch umgekehrt durchgeführt werden. Die Ursache für das Auftreten dieser Widerstandsänderungen wird in der Serienschaltung zweier identischer, asymmetrischer EPIR-Kontakte gesehen. Chen et al. gehen davon aus, dass die Widerstandsänderungen vornehmlich an den Grenzflächen auftreten. Sie unterteilen demnach den symmetrischen Kontakt in zwei gespiegelte EPIR-Kontakte, wobei die mikroskopische Ursache der Widerstandsänderung nicht näher spezifiziert wird. Befinden sich beide Halbkontakte im Ausgangszustand im hochohmigen Zustand (dieser Zustand wird in den Messungen nie beobachtet, lediglich in Simulationen), so geht einer der beiden für positive, hohe Spannungen in den niederohmigen Zustand über. Dieser Zustand sollte nun für alle Spannungen stabil sein, allerdings ist die Serienschaltung der Halbkontakte zu beachten. Da sich ein Halbkontakt im hochohmigen, der andere im niederohmigen Zustand befindet, ist der Spannungsabfall an den Halbkontakten nicht identisch. Dadurch können beide Halbkontakte in einem gewissen Spannungsbereich in den niederohmigen Zustand gebracht werden.

Das von Chen et al. beobachtete Verhalten kann nicht nur die Widerstandsänderungen in hochsymmetrischen Kontakten erklären, sondern liefert auch eine mögliche Interpretation dafür, dass für den breiten Kontakt ein erstes Widerstandsschalten erst für negative Spannungen auftritt, obwohl eine positive Spannung gleichen Betrages zunächst keinen Einfluss zeigt. Stellt der Ausgangszustand eine Konfiguration dar, in welcher jeweils ein 
Halbkontakt im hochohmigen, bzw. niederohmigen Zustand vorliegt, so ist eine bestimmte Polarität zur Widerstandserniedrigung notwendig. Für die gegensätzliche Polarität wäre keine Widerstandsänderung zu beobachten. Auch das Verhalten beim Ändern der Anschlüsse an der Stromquelle folgt direkt aus diesem Modell.

Aus der Art der in dieser Arbeit vorgenommenen Versuchsdurchführung folgt, warum der niederohmige Zustand auch für hohe Stromstärken stabil ist und nur die so genannten minor loops gemessen werden. In den Experimenten wird im Unterschied zur üblichen Durchführung von EPIR-Untersuchungen die Stromstärke symmetrisch um $I=0$ variiert, woraus bei auftretenden Widerstandsänderungen keine symmetrische Spannungsvariation folgt. Zur Illustration sei beispielsweise auf die Strom-Spannungs-Kennlinie in Abbildung 6.17 verwiesen: Nachdem eine ausreichend hohe Spannung auch den zweiten Halbkontakt in den niederohmigen Zustand gebracht hat, wäre nur eine kleine Spannungszunahme nötig, um den anderen Halbkontakt in den hochohmigen Zustand zu bringen. Aufgrund der massiven Widerstandsabnahme des gesamten Kontaktes übersteigt die abfallende Spannung aber im positiven Bereich die nötige Spannung nicht mehr. Eine Stromstärke von mehr als $500 \mu \mathrm{A}$ wäre bei Annahme eines konstanten Widerstandes für zunehmende Spannung notwendig, damit die abfallende Spannung gegenüber den Werten im hochohmigen Zustand ansteigt. Insofern ist die stromkontrollierte Durchführung als Möglichkeit zur einfachen Bestimmung von minor loops anzusehen.

Zur Bestätigung dieser Hypothese werden die in Abbildung 6.23 dargestellten Experimente durchgeführt: Nach Erreichen des niederohmigen Zustandes wird die Stromstärke dahingehend weiter erhöht, dass die abfallende Spannung, welche zunächst aufgrund der Widerstandsabnahme gegenüber dem hochohmigen Zustand verringert ist, einen neuen Maximalwert annimmt. Die Ergebnisse zeigen, dass sich unter Vernachlässigung von Bereich I, also der kontinuierlichen Widerstandsabnahme im hochohmigen Zustand, eine qualitativ hervorragende Übereinstimmung mit den in Abbildung 6.26 a) dargestellten Ergebnissen von Chen et al. ergibt. Nach einem ersten Erreichen des niederohmigen Zustandes wird der hochohmige Zustand widerhergestellt, falls die im niederohmigen Zustand abfallende Spannung die des hochohmigen Zustandes überschreitet. Dies kann als weiteres Indiz dafür gewertet werden, dass die Spannung, bzw. das elektrische Feld ursächlich für das Widerstandsschalten ist.

Durch das oben entworfene Bild für die asymmetrischen Widerstandsänderungen können die Schwankungen des niederohmigen Zustandes für unterschiedliche Messungen erklärt werden: Für EPIR-Experimente ist bekannt, dass sich der Widerstand zwar nur in einem kleinen Spannungsfenster ändert, diese Änderung aber kontinuierlich ist [88. Somit sind Zwischenzustände zugänglich. Führt nun ein Strom zu einer Widerstandsänderung, welche nicht im tiefsten zugänglichen Widerstand endet, so muss ein deutlich höherer Strom durch den Kontakt fließen, um durch eine Erhöhung des elektrischen Feldes den tiefstmöglichen Widerstandszustand zu erreichen. Anderenfalls kommt es zu Schwankungen des niederohmigen Zustandes. Dieses wird teilweise auch an Abbildung 6.23 a) deutlich. 


\section{Mikroskopische Modellentwicklung}

Das oben diskutierte Modell von Chen et al. beschreibt das in dieser Arbeit beobachtete Widerstandsverhalten offenbar recht gut, dennoch bleibt die Frage nach einem mikroskopischen Mechanismus offen. Eine einfache Erklärung durch Grenzflächeneffekte an den Elektroden konnte im vorliegenden System weitgehend ausgeschlossen werden. Daher motiviert die gelungene Beschreibung des Widerstandsverhalten durch das Modell von Chen et al. die Suche nach einem System, welches in zwei verschiedenen Zuständen einen identischen Widerstand aufweist. Im Modell des PCMO-Kontaktes besteht dieses aus jeweils einem hoch- und einen niederohmigen Halbkontakt, deren zwei Kombinationsmöglichkeiten einen identischen Widerstand ergeben. Wie bereits oben diskutiert wurde, ist im nanokristallinen LSMO bei tiefen Temperaturen vermutlich eine Modifikation der Korngrenzen für das Verhalten verantwortlich. Im Folgenden wird eine stukturelle Änderung des Manganats in unmittelbarer Nähe zur Korngrenze als möglicher Mechanismus diskutiert. Die Argumentation basiert auf folgenden Überlegungen:

- Die Widerstandsänderungen bleiben zumindest gelegentlich auch für kleine Stromstärken erhalten. Dies deutet auf eine strukturelle Änderung hin, welche auch ohne elektrisches Feld aufrecht erhalten werden kann. Ein rein elektronischer Mechanismus würde unter Verringerung des Feldes immer in den gleichen Zustand zurückkehren.

- Die oben geführte Diskussion der Widerstandsänderungen legt einen auf den Korngrenzen basierenden Mechanismus nahe, wodurch die effektive Barrierenbreite verringert wird.

- Die elektronischen Eigenschaften der Korngrenzen werden häufig in Zusammenhang mit mechanischen Spannungsfeldern diskutiert [28], wobei ein gestörter Bereich in der Nähe der Korngrenze vermutet wird. Die mechanischen Spannungen haben dabei eine gewisse laterale Ausdehnung und sind nicht auf eine Atomlage beschränkt |204|.

- Bereits im System PCMO konnten direkte Hinweise für strukturelle Änderungen unter anderem in Form von korrelierter Rotation der $\mathrm{MnO}_{6}$-Oktaeder als Ursache für massive Widerstandsänderungen unter Einfluss eines Stromes bzw. elektrischen Feldes gefunden werden [22].

Abbildung 6.27 zeigt ein idealisiertes Strukturmodell, welches die beobachteten Widerstandsänderungen insbesondere unter Berücksichtigung der Symmetrieüberlegungen erklären kann. Es beruht auf der Annahme, dass es im Grundzustand in der Nähe der Korngrenze zu zwei isostrukturellen Abweichungen ( $B$ und $B^{\prime}$ ) gegenüber einer dem Korninneren ähnlichen Struktur $(A)$ kommt, in denen die $\mathrm{MnO}_{6}$-Oktaeder stärker verkippt sind. Entsprechend weisen die Strukturen $B$ und $B$ ' aufgrund ihrer Symmetrie einen identischen, gegenüber der Struktur $A$ infolge der unterschiedlichen Mn-O-Mn Bindungswinkel aber deutlich erhöhten Widerstand auf. Die Struktur $A$ hat nicht zwangsläufig eine identische Struktur und Leitfähigkeit wie das Korninnere, soll aber nicht stark davon 


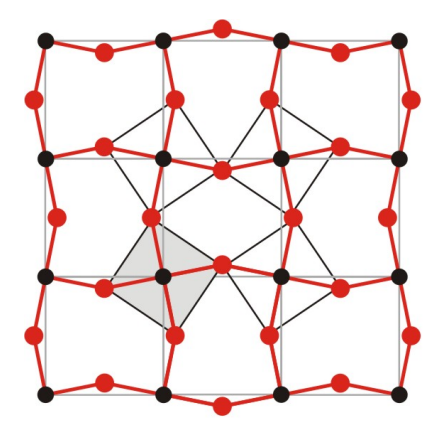

B

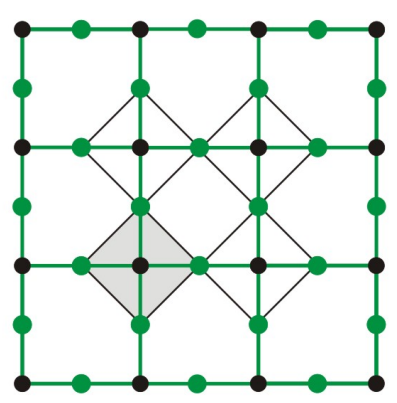

A

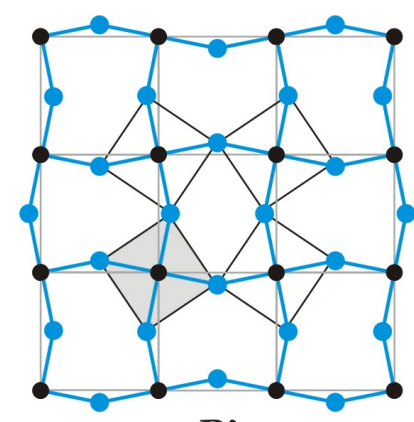

B'

Abbildung 6.27. Mögliche strukturelle Konfigurationen des LSMO in der Nähe der Korngrenze (idealisiert). Dargestellt sind lediglich die Mn-Ionen (schwarz) und die O-Ionen (farbig) in der $a$ - $b$-Ebene. Die Transformation B $\rightarrow$ B', bzw. B' $\rightarrow$ B erfolgt über den leitfähigeren Zustand A. Die Hervorhebung jeweils eines Oktaeders dient der Unterscheidung der Strukturen (s. Text).

abweichen. Im Weiteren werden deshalb zur Vereinfachung der Diskussion die Strukturen als identisch angesehen.

Die Potentiallandschaft ist gemäß Abbildung 6.28 an der Korngrenze gegenüber dem Korninneren geändert. Während im Korninneren nur das globale Minimum der Struktur $A$ vorhanden ist, treten in der Nähe der Korngrenze weitere Minima der Konfigurationen $B$ und $B$ ' auf. Diese beiden Modifikationen sind durch eine Potentialbarriere voneinander getrennt. Abbildung 6.27 zeigt aber, dass eine Überführung der Struktur $B$ in $B^{\prime}$ immer über die im Korninneren stabile Konfiguration $A$ geschieht. Aufgrund der Stabilität im Korninneren kann angenommen werden, dass sie in der Potentiallandschaft ein kleines, lokales Minimum verursacht.

Die wesentliche Annahme dieses mikroskopischen Modells besteht darin, dass ausgehend vom Zustand $B\left(B^{\prime}\right)$ das elektrische Feld bei einem kritischen Wert zu einer Rotation der $\mathrm{MnO}_{6}$-Oktaeder zunächst in die Struktur $A$, und bei einer weiteren Erhöhung in die isostrukturelle Modifikation $B^{\prime}(B)$ führt. Wird nur ein einzelnes Oktaeder betrachtet (s. beispielsweise das schattierte Oktaeder in Abb. 6.27), so hat dieses einen definierten Drehsinn bezüglich einer vorgegebenen Richtung eines elektrischen Feldes. Eine Feldumkehr führt entsprechend zu einer Rotation in die entgegengesetzte Richtung.

Durch die Transformationen zwischen den strukturellen Modifikationen $A$ und $B$ bzw. $B$ ' kann nun das experimentell ermittelte Widerstandsverhalten erklärt werden. Ausgehend von einem System, in welchem der Bereich der Korngrenzen zunächst in der Konfiguration $B$ vorliege, wird dieser Bereich durch ein kritisches elektrisches Feld definierter Polarität in die Konfiguration $A$ transformiert. Somit wird ein wesentlicher Anteil des gestörten Bereiches leitfähiger, die Barrierenbreite nimmt ab und das System wechselt in den niederohmigen Zustand. Eine weitere Erhöhung des elektrischen Feldes führt dazu, dass auch dieser Zustand instabil wird und das System in die Konfiguration $B$ ' wechselt. Die Barrierenbreite und folglich auch der Widerstand nimmt wieder zu, das System befindet sich im hochohmigen Zustand, welcher aufgrund der Symmetrie der Strukturen $B$ und $B^{\prime}$ durch eine reine Widerstandsbestimmung nicht zu unterscheiden ist. Für eine 


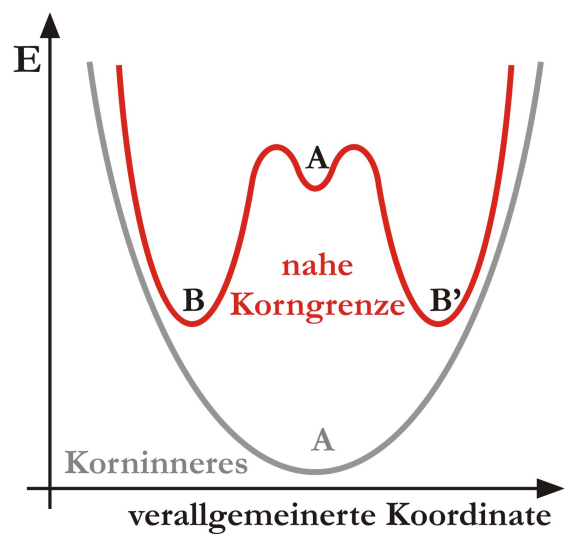

Abbildung 6.28. Mögliche Potentiallandschaft (schematisch) in der Nähe der Korngrenze (rot), bzw. im Korninneren (grau).

Umkehr der Polarität zeigt sich das symmetrische Verhalten mit einer Transformation $B$ ' $\rightarrow A \rightarrow B$. In Abbildung 6.29 a) wird dieses Szenario auf die in dieser Arbeit erzielten Ergebnisse transferiert. Die Farbkodierung wird analog zu Abbildung 6.27 gewählt. Da nur der qualitative Verlauf beschrieben wird und anschließend das asymmetrische Verhalten analysiert werden soll, werden willkürliche Einheiten gewählt.

Wird das elektrische Feld nach Erreichen des metastabilen Zustandes $A$ nicht weiter erhöht, sondern stattdessen erniedrigt und schließlich die Polarität geändert, so wechselt das System bei einem kritischen Feld zurück in die Konfiguration B. Dieser Mechanismus könnte das ursprünglich beobachtete, asymmetrische Verhalten erklären, siehe Abbildung $6.29 \mathrm{~b})$.

Weiterhin könnte es in kleinen elektrischen Feldern gegebenenfalls zu Relaxationen des Zustandes $A$ in unterschiedliche Volumenanteile der Konfigurationen $B$ und $B^{\prime}$ kommen. Dies würde zunächst zu Widerstandserhöhungen in kleinen elektrischen Felder führen. Träte ein genügend großer Volumenanteil der Konfiguration $B^{\prime}$ auf, so würde dieser aber zunächst in die leitfähigere Struktur $A$ überführt, bevor der Zustand $B$ stabil wird. Entsprechend würde es bei Verringerung der elektrischen Feldstärke aus dem beispielsweise positiven Maximalwert zunächst zu einer Erhöhung des Widerstandes kommen (Relaxation von $A$ in $B$ und $B^{\prime}$ ), dann zu einer Abnahme des Widerstandes ( $B^{\prime}$ in $A$ ) und schließlich zu einer Zunahme $(A$ in $B$ ). Dieses Verhalten entspräche Bereich III. Ist der relaxierte Volumenanteil aber nur klein, so sind keine wesentlichen Widerstandsvariationen im niederohmigen Zustand zu erwarten. Für diese Annahme spricht, dass die Leitfähigkeit im niederohmigen Zustand nur sehr unbefriedigend mit dem Modell nach Simmons (s. Kap. 3.1.3 beschrieben werden kann. Würde jedoch lediglich die Barrierenbreite geändert und zeitliche Effekte keine Rolle spielen, so wäre dieses weiterhin zu erwarten. Neben den symmetrischen und asymmetrischen Widerstandsänderungen kann das Modell also gegebenenfalls auch das temporäre Auftreten des Bereiches III erklären.

Obwohl das hier entworfene, idealisierte Strukturmodell eine mögliche Erklärung der beobachteten Widerstandsänderungen darstellt, existiert keinerlei experimentelle Evidenz für dieses Modell. Zudem bleibt der genaue Wechselwirkungsmechanismus mit dem 

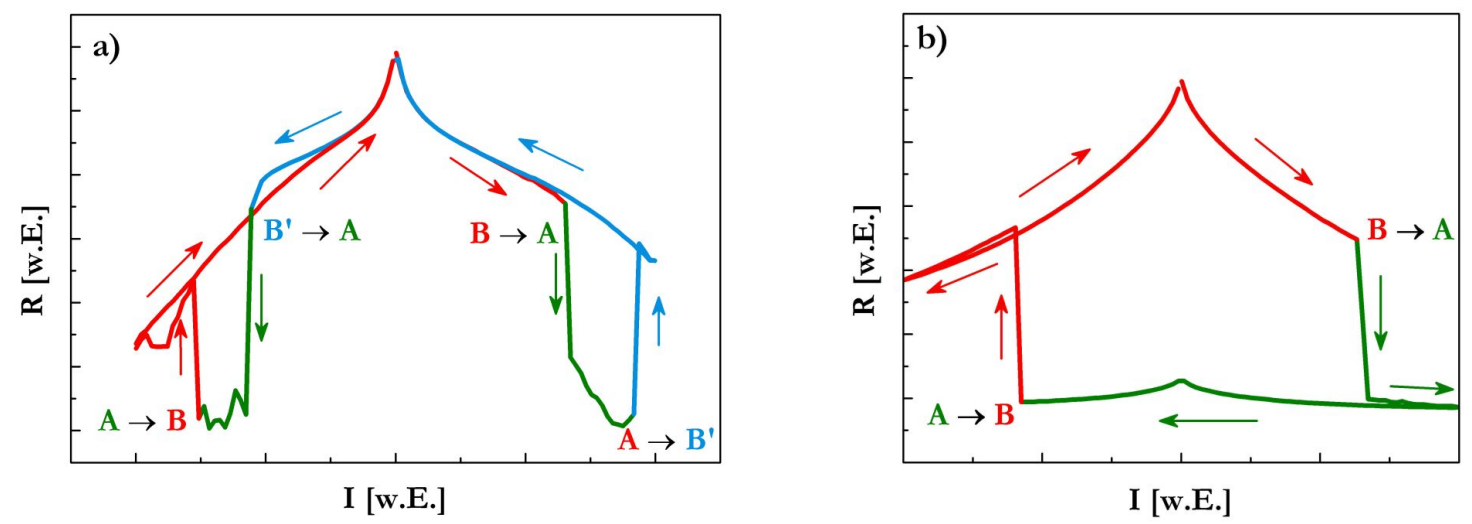

Abbildung 6.29. Übertragung des idealisierten Strukturmodells auf die experimentell erzielten Ergebnisse. Die Farbkodierung ist Abbildung 6.27 entnommen. Zum Vergleich der an zwei unterschiedlichen Proben gewonnenen Resultate werden die Absolutwerte verworfen.

elektrischen Feld weiter unklar. Ein wichtige Rolle spielt in diesem Zusammenhang vermutlich die Brechung der Symmetrie an der Korngrenze [205]. Anderenfalls ergeben sich Schwierigkeiten in der Interpretation der Wechselwirkung aufgrund der zueinander symmetrischen Strukturen $B$ und $B^{\prime}$ : So wird zwar für jedes einzelne Oktaeder eine definierte Drehrichtung bezüglich der Feldrichtung angenommen, allerdings existieren beispielsweise in den beiden Konfigurationen $B$ und $B^{\prime}$ gleichberechtigte Oktaeder, welche einerseits in einem positiven, andererseits in einem negativen elektrischen Feld im Uhrzeigersinn rotieren. Dabei ist jedoch zu beachten, dass das angegebene Strukturmodell idealisiert ist, die genauen Strukturen also nicht spezifiziert sind.

\section{Offene Fragen}

Eine grundsätzliche Erklärung der beobachteten Widerstandsänderungen gelingt durch die bisher diskutierten Mechanismen offensichtlich gut. Dennoch ergeben sich offene Fragestellungen. So ist die Notwendigkeit der Strukturierung ungeklärt, da Referenzexperimente an unstrukturierten Proben fehlen. Auch die Bestimmung der Temperaturabhängigkeit der Prozesse könnte weitere Einblicke in die mikroskopischen Ursachen liefern. Dabei bietet das hier untersuchte System Vorteile gegenüber anderen Systemen wie PCMO, da die Tieftemperaturphase in einem weiten Temperaturintervall stabil ist. Im PCMO dagegen sind weitere Effekte wie das Einsetzen der Ladungs- und Orbitalordnung zu beachten. Die hier untersuchten Temperaturen weichen nur wenig voneinander ab $(\Delta T=17 \mathrm{~K})$, wobei kein Unterschied in den wesentlichen Merkmalen der Messungen festgestellt wird. Weiterhin ist das Verhalten in Bereich III im Wesentlichen ungeklärt.

\section{Mögliches Anwendungspotenzial}

Der EPIR-Mechanismus wird oft im Zusammenhang mit möglichen Anwendungen wie RRAMs (engl.: resistive random access memory) genannt [26]. Die stabilen Widerstandszustände bilden dabei nichtflüchtige Informationsspeicher und haben somit gegenüber konventionellen, flüchtigen Speichern Vorteile. Das hier untersuchte System bietet neben 
den beiden stabilen Widerstandszuständen, welche durch das elektrische Feld definiert werden, weitere definierte Widerstandsvariation durch das magnetische Feld auf Grundlage des LFMR. Insgesamt sind somit aufgrund der unabhängigen Wirkung der verschiedenen äußeren Felder vier Widerstandszustände zugänglich (s. Abb. 6.20). Die erfolgreiche Präparation von Kontakten mit vier definierten Zuständen wird als Durchbruch auf der Suche nach verbesserten Speichermedien gesehen 206. Gajek et al. berichten von einer erfolgreichen Präparation magnetischer Tunnelkontakte unter Verwendung einer multiferroischen Barriere und verfolgen demnach ebenso einen Ansatz, in dem der Widerstand durch eine Kombination von elektrischen und magnetischen Feldern beeinflusst wird [207]. Durch Anlegen eines Spannungspulses von $\pm 2 \mathrm{~V}$ können die $R(H)$-Kurve, welche einen TMR-Effekt von bis zu $20 \%$ aufweisen, um $\Delta R \approx 30 \mathrm{k} \Omega$ relativ zueinander verschoben werden. So entstehen theoretische vier Widerstandszustände. Dabei führt jedoch der TMR, welche gerade eine Widerstandsänderung von ca. $30 \mathrm{k} \Omega$ darstellt, effektiv zu nur drei Widerstandszuständen [207]. Weiterhin ist aufgrund des multiferroischen Charakters der Tunnelbarriere die Wirkung der verschiedenen äußeren Felder nicht unabhängig voneinander [206, 207].

Die hier gewonnenen Ergebnisse stellen demnach eine deutliche Verbesserung gegenüber den Ergebnissen von Gajek et al. dar. Die vier zugänglichen Widerstandszustände sind klar voneinander getrennt und auch die Wirkung der äußeren Felder auf den Widerstand scheint unabhängig voneinander zu sein. Auch wenn die durch das magnetische Feld erzeugten Widerstandsvariationen im speziellen Fall flüchtig sind, ergeben sich dennoch neue Möglichkeiten in der Nanotechnologie 92 . 



\section{Kapitel 7}

\section{Bikristallkontakte}

Wie im vorherigen Kapitel gezeigt, können die Eigenschaften von Manganaten durch das kontrollierte Einbringen von (dotierten) Korngrenzen gezielt beeinflusst werden. Bikristallkontakte bieten dabei die Möglichkeit, den Einfluss einer einzelnen, definierten Korngrenze auf das Verhalten des zu untersuchenden Systems zu charakterisieren.

In diesem Kapitel werden drei unterschiedliche Systeme vorgestellt und diskutiert: Einerseits undotierte und MgO-dotierte LSMO Bikristallkontakte, welche sowohl auf STOund $\mathrm{MgO}-$ Substraten deponiert werden, anderseits ein LSMO: $\mathrm{CeO}_{2}$ Bikristallkontakt auf STO. Zunächst wird die Struktur der präparierten Schichten untersucht (Kap. 7.1), wobei auch TEM-Querschnittsanalysen zum Einsatz kommen. Anschließend erfolgt eine Untersuchung der Transporteigenschaften (Kap. 7.2) unter besonderer Berücksichtigung der Kontaktbreite über die Korngrenze. Schließlich werden strominduzierte Widerstandsänderungen in Bikristallkontakten vorgestellt (Kap. 7.3.

Die Schichten werden mittels MAD auf symmetrischen, 24 [001]-tilt BikristallSubstraten (s. Kap. 5.1.2) deponiert. Die Dicke der hergestellten Schichten beträgt dabei ca. $50 \mathrm{~nm}$, was wie bei der Präparation der Nanokomposite über das Lösungsvolumen eingestellt und anschließend durch Röntgenreflektometrie kontrolliert wird. Falls nicht weiter angegeben beträgt die Zusammensetzung der Schichten im MgO-dotierten Fall $(\mathrm{LSMO})_{0,9}:(\mathrm{MgO})_{0,1}$ und im $\mathrm{CeO}_{2}$-dotierten Fall $(\mathrm{LSMO})_{0,8}:\left(\mathrm{CeO}_{2}\right)_{0,2}$.

\subsection{Struktur}

Die strukturellen Eigenschaften der Proben werden mittels Röntgendiffraktometrie und REM-Aufnahmen charakterisiert. Abbildung 7.1 a) zeigt das Röntgendiffraktogramm einer auf einem STO-Substrat deponierten LSMO-Probe. Alle Reflexe können unter Verwendung des Substrats und einer LSMO-Phase indiziert werden. Dabei ergeben sich ausschließlich Reflexe des Typs (00l). In Abbildung 7.1 b) ist der (002)-Reflex im Detail dargestellt. Neben der $\mathrm{K}_{\alpha, 1}-\mathrm{K}_{\alpha, 2}$-Aufspaltung des Substratreflexes ist der LSMO (002)-Reflex zu erkennen, über dessen Lage der c-Achsen-Gitterparameter zu $c=3,854 \AA$ berechnet werden kann.

Das gezeigte Verhalten ändert sich nicht durch die Dotierung der Proben mit MgO. 

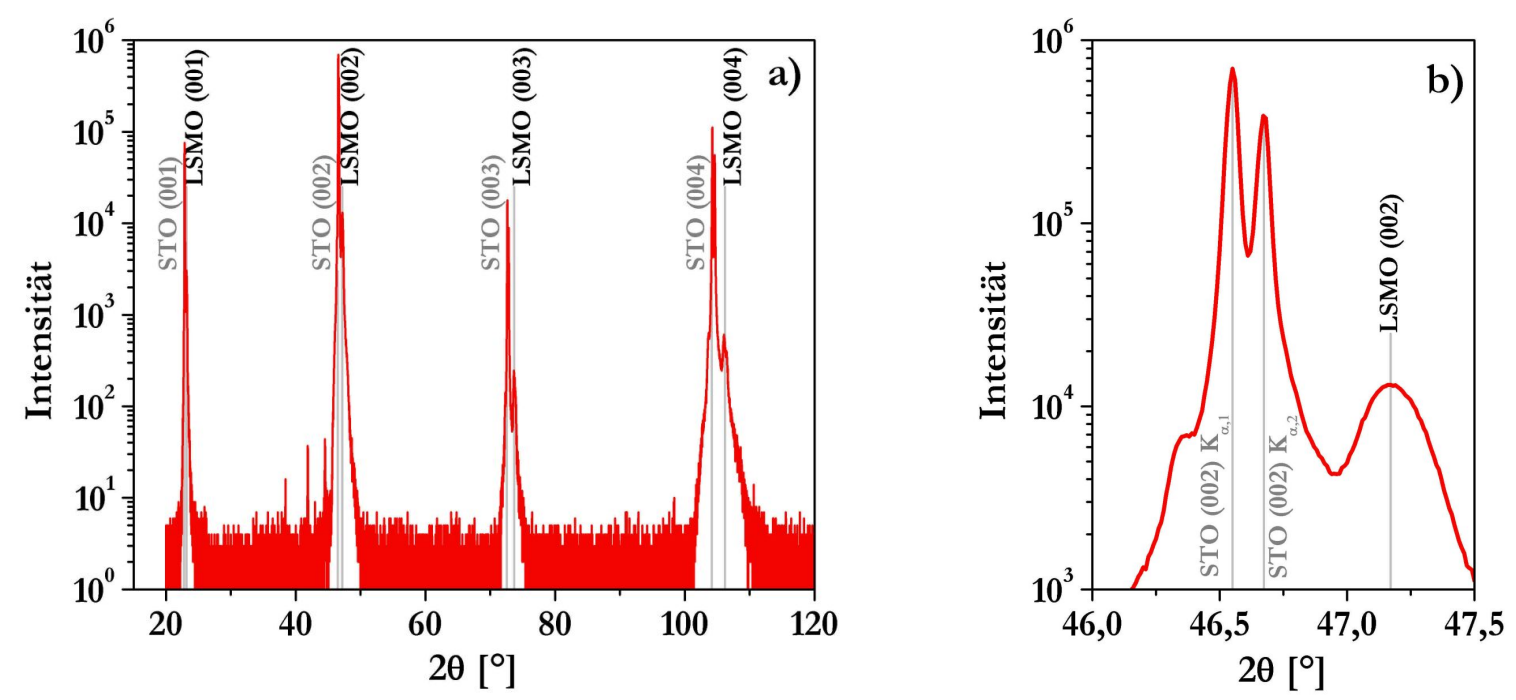

Abbildung 7.1. a) Röntgenstrukturanalyse einer LSMO-Schicht deponiert auf einem STOBikristall. b) Detail-Ausschnitt von a).

Weiterhin treten nur Substrat- und LSMO-Reflexe des Typs (00l) auf, MgO-Reflexe sind im Rahmen der Messgenauigkeit nicht auszumachen. Die Lage des LSMO (002)Reflexes verschiebt sich leicht zu kleineren Winkeln, der c-Achsen-Gitterparameter beträgt $c=3,858 \AA$. Im Falle der $\mathrm{CeO}_{2}$-Dotierung hingegen tritt neben den Substrat- und LSMOReflexen zusätzlich der $\mathrm{CeO}_{2}$ (002)-, bzw. (004)-Reflex auf. Der zugehörige Gitterparameter beträgt $c=5,41 \AA$. Weiterhin verschiebt sich die Lage des LSMO (002)-Reflexes in Bezug auf den undotierten Fall zu leicht höheren Winkeln, der c-Achsen Gitterparameter ergibt sich zu $c=3,851 \AA$.

Für die Schichten auf MgO-Bikristallen ergibt sich ein ähnliches Bild. Wiederum treten nur Substrat- und LSMO-Reflexe mit (00l)-Orientierung auf. Der c-AchsenGitterparameter ergibt sich sowohl für die undotierten als auch für die MgO-dotierten Proben zu $c=3,886 \AA$.

REM-Aufnahmen der Oberfläche einer auf STO deponierten LSMO-Schicht sind in Abbildung 7.2 dargestellt. Beide Aufnahmen, deren Abstand auf der Oberfläche einige Mikrometer beträgt, beinhalten die Korngrenze, welche jeweils vertikal verläuft. Beidseitig der Korngrenze sind auf der Oberfläche rechteckige Strukturen mit einer mittleren Größe von 40-50 nm zu erkennen. Diese Strukturen weisen dabei eine bevorzugte Orientierung in Bezug auf die Korngrenze auf: Eine Seite der rechteckigen Strukturen schließst mit der Korngrenze einen Winkel von ca. $12^{\circ}$ ein. Diese Vorzugsrichtung weist eine Spiegelsymmetrie auf, wobei die Korngrenze in der Spiegelebene liegt. Demnach zeigen die beobachteten Strukturen die gleiche Symmetrie wie das Substrat auf. Die räumliche Ausdehnung der Korngrenze liegt meist unterhalb des Auflösungsvermögen des REM, allerdings zeigt besonders Abbildung 7.2 b), in Teilen aber auch $7.2 \mathrm{a}$ ), dass die Korngrenze strukturell inhomogen ist. Es treten Löcher mit einer Ausdehnung von bis zu $300 \mathrm{~nm} \times 80 \mathrm{~nm}$ auf. Die Form orientiert sich dabei an den rechteckigen Strukturen der Oberfläche: Der Öffnungswinkel beträgt ca. $24^{\circ}$ und verläuft parallel zu den Kanten der Strukturen. Diese Löcher 

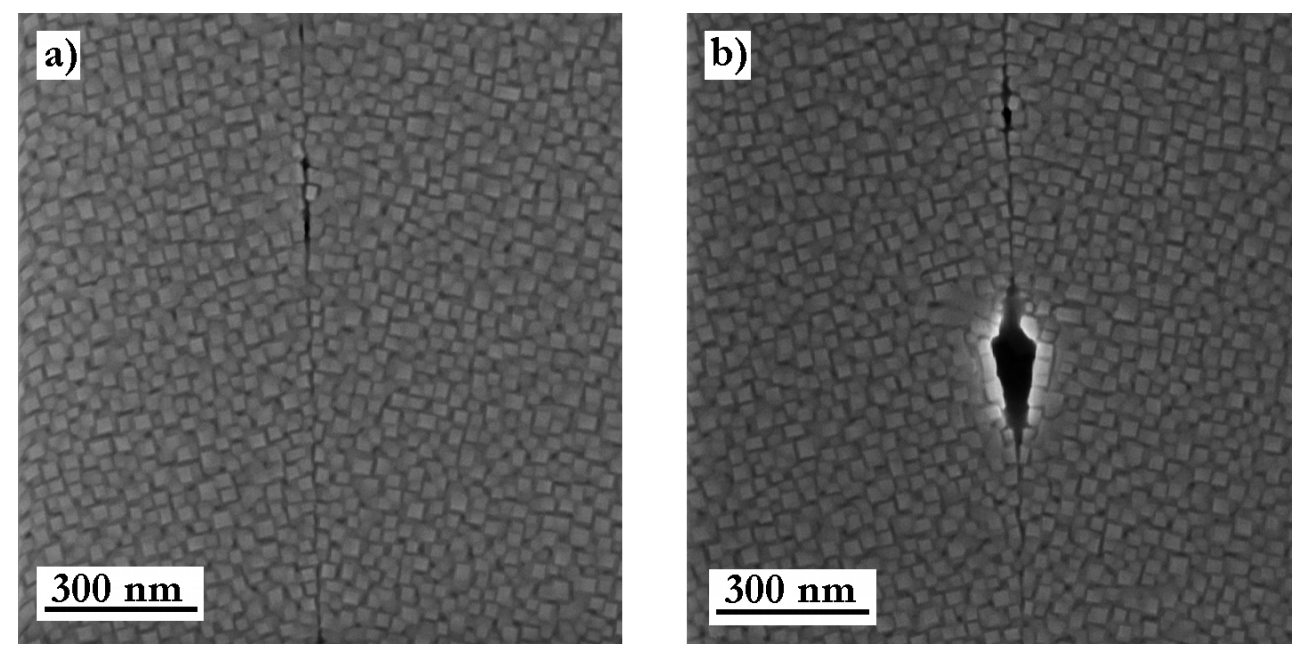

Abbildung 7.2. REM-Aufnahmen einer LSMO-Probe auf einem STO-Bikristall Substrat. Die untersuchten Stellen a) und b) liegen wenige Mikrometer auseinander.

zeigen kein systematisches Auftreten entlang der Korngrenze. So finden sich Bereiche in der Größenordnung von mehreren Mikrometern, in denen diese Inhomogenitäten nicht auftreten. Andererseits existieren Bereiche, in denen der Abstand dieser Löcher ca. $1 \mu \mathrm{m}$ beträgt.

Die in Abbildung 7.2 dargestellten Oberflächenstrukturen sind repräsentativ für alle Bikristallkontakte. Für alle Proben wird eine regelmäßige Anordnung der rechteckigen Strukturen beidseitig der Korngrenze gefunden, der Winkel zur Korngrenze beträgt immer ca. $12^{\circ}$. Auch die strukturellen Inhomogenitäten auf der Längenskala einiger Mikrometer finden sich bei allen Proben. Ein systematisches Auftreten entlang der Korngrenze wird aber für keine Probe gefunden.

Zur weiteren strukturellen Charakterisierung wird von einer LSMO-Probe, präpariert auf einem MgO-Bikristall Substrat, eine Querschnitts-TEM-Analyse angefertigt. Hierzu wird mittels FIB eine Lamelle präpariert (Breite ca. $150 \mathrm{~nm}$ ), welche annähernd senkrecht zur Korngrenze verläuft. In dieser Geometrie ist es unmöglich, den Elektronenstrahl für beide Substrathälften gleichzeitig entlang einer niedrig indizierten Kristallrichtung zu orientieren. Dieses wäre nur für eine Aufsicht zu realisieren, wobei dann die relativ einfache Lamellenpräparation mittels FIB nicht mehr durchführbar ist. Da die Elektronenstrahlrichtung relativ zur Probenoberfläche um ca. $\pm 15^{\circ}$ variiert werden kann, ist es möglich, nacheinander beide Substrathälften so auszurichten, dass ihre [010]-Richtungen parallel zum Elektronenstrahl liegen.

Abbildung 7.3 zeigt Querschnitts-TEM-Aufnahmen vom Rand der Lamelle (a) ohne Korngrenze und von der Mitte der Lamelle (b) mit Korngrenze. Es sind jeweils drei Bereiche zu erkennen: In der oberen, linken Ecke befindet sich die im FIB mittels Elektronenstrahl deponierte Pt-Schutzschicht. Nach unten rechts folgt zunächst die LSMOSchicht und schließlich das MgO-Substrat. In Abbildung a) ist zu erkennen, dass die Oberfläche verhältnismäßig glatt ist, an einer Stelle aber eine Unregelmäßigkeit aufweist: Die Schichtdicke verringert sich um ca. 4 Einheitszellen. Solche Sprünge (in beide Rich- 

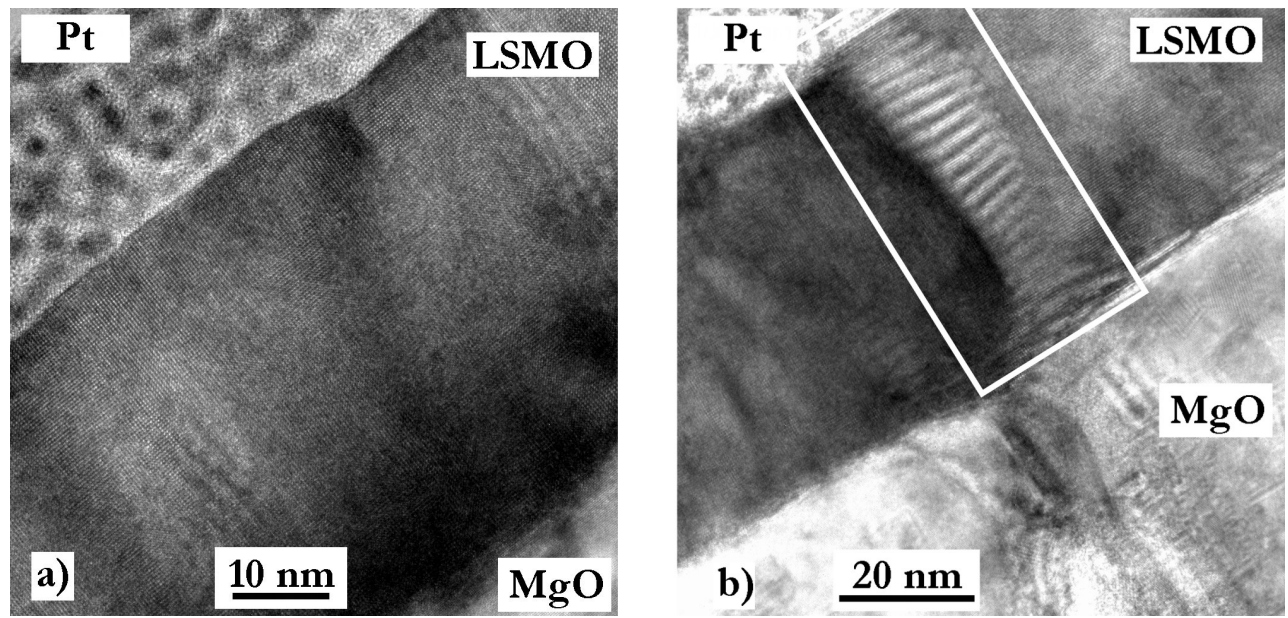

Abbildung 7.3. TEM-Querschnittsaufnahmen einer LSMO-Probe auf einem MgOBikristall Substrat. a) Ausschnitt ohne Korngrenze an der Seite der Lamelle. b) Ausschnitt mit Korngrenze in der Mitte der Lamelle (durch Umrahmung hervorgehoben).

tungen) wiederholen sich in einen Abstand von ca. $50 \mathrm{~nm}$. Wie in der Abbildung aber ersichtlich wird, sind sie nicht mit einem durch die Probe laufenden Defekt verbunden. Im abgebildeten Ausschnitt zeigen sich keine ausgedehnten Defekte, die Anordnung der Atome ist regelmäßig.

Die Korngrenze ist in Abbildung $7.3 \mathrm{~b}$ ) abgebildet. Dabei ist der linke Halbkristall entlang der Elektronenstrahlrichtung orientiert. Die Lamelle ist also um $12^{\circ}$ relativ zum Strahl verkippt, der rechte Halbkristall demnach um $24^{\circ}$ : Entsprechend ist keine atomare Auflösung für diesen Teil zu erreichen, es sind lediglich parallel zum Substrat verlaufende Streifen mit dem Abstand einer Einheitszelle zu beobachten. Die Korngrenze in der Schicht ist durch den zusätzlichen weißen Rahmen markiert. Allerdings lässt die Dicke der Lamelle an gerade dieser Stelle sowie die überlappenden Halbkristalle eine hochauflösende Analyse nicht zu. Weiterhin kann auch keine quantitative Aussage über die räumliche Ausdehnung der Korngrenze getroffen werden. Die Region, in welcher breitere Streifen mit einem Hell-Dunkel-Kontrast parallel zum Substrat verlaufen, hat eine Ausdehnung von ca. $15 \mathrm{~nm}$. Dieses entspricht im Wesentlichen der Projektion der senkrecht durch die Lamelle verlaufenden Korngrenze auf eine zum Elektronenstrahl senkrechte Ebene. Dies stellt somit die obere Grenze für die Breite der Korngrenze dar, welche aber sicher schmaler ist.

Das TEM-Bild 7.3 b) zeigt außerdem, dass die Korngrenze im Substrat in der Nähe der Oberfläche nicht sauber definiert ist. Die Halbkristalle zeigen in einem Bereich größer als $20 \mathrm{~nm}$ keine regelmäßigen Strukturen auf, was nicht durch einen Überlapp der Halbkristalle erklärt werden kann.

Zur weiteren Analyse der Korngrenze wird eine Mikroanalyse mittels Nano-EDX durchgeführt (s. Kap. 5.6). Abbildung 7.4 zeigt die relative Intensität der charakteristischen Röntgenstrahlung der La $L$-Linie und der Mg $K$-Linie als Funktion des Ortes. Der Elektronenstrahl wird dabei parallel zur Substratoberfläche über die LSMO-Probe gerastert und quert die Korngrenze (siehe Einsatz von Abbildung 7.4). Im Bereich der 


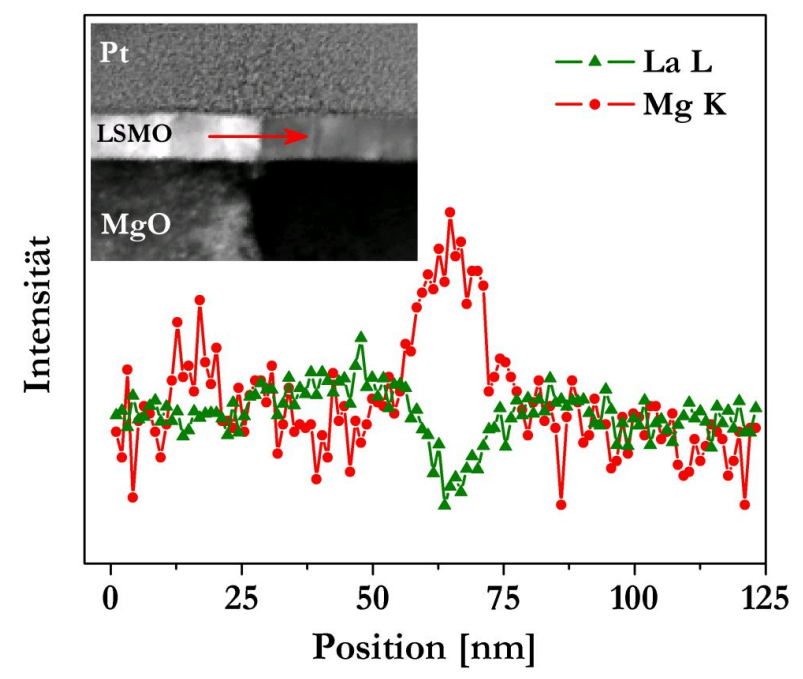

Abbildung 7.4. Mikroanalyse der Korngrenze mittels Nano-EDX. Der rote Pfeil im TEMBild gibt die Rasterrichtung des Elektronenstrahls wider. Die Lage der Korngrenze lässt sich besonders am Hell-Dunkel-Kontrast in der LSMO-Schicht erkennen.

Korngrenze ist über einen Bereich von 15-20 nm eine signifikante Erhöhung des Mg-Signals zu beobachten, welche einhergeht mit einer Abnahme des La-Signals.

\subsection{Magnetotransport}

Im Folgenden wird der Magnetotransport der Bikristallkontakte näher betrachtet. Dabei werden insbesondere die Ergebnisse der auf STO-deponierten Proben präsentiert. Zunächst werden die Ergebnisse der reinen LSMO-Schichten vorgestellt, anschließend die der $\mathrm{MgO}$-dotierten und letztlich die des $\mathrm{CeO}_{2}$-dotierten LSMO-Kontaktes.

\subsubsection{LSMO Korngrenzen-Kontakte}

Die Verwendung der in Kapitel 5.5.1 definierten Mikrostruktur 1 erlaubt die gleichzeitige Bestimmung des elektrischen Widerstandes sowohl über die Korngrenze hinweg als auch ohne Einfluss der künstlichen Korngrenze auf dem Halbkristall. Im Weiteren wird diese zweite Messung mit epitaktisch bezeichnet. Der Vergleich der beiden normierten, temperaturabhängigen Widerstandsverläufe ist gemeinsam mit der normierten Magnetisierung für eine LSMO-Probe auf einem STO-Substrat in Abbildung 7.5 a) dargestellt. Der Widerstand wird dabei mit $I=7 \mu \mathrm{A}$, die Magnetisierung in einem kleinen äußeren Feld von $H=100$ Oe ermittelt. Die Magnetisierungskurve offenbart ein ferromagnetisches Verhalten mit einem vergleichsweise scharfen Phasenübergang bei $T_{C}=350 \mathrm{~K}$, bestimmt durch die Lage des Minimums in $(1 / M)(\partial M / \partial T)$. Die Widerstandsmessung ohne Einfluss der Korngrenze zeigt einen typischen Verlauf für epitaktisch gewachsenes LSMO. Im gesamten Temperaturbereich, auch oberhalb von $T_{C}$, gilt $d R / d T>0$, es liegt ein metallisches Verhalten vor. In der Nähe von $T_{C}$ zeigt sich dabei ein starker Anstieg des Widerstan- 

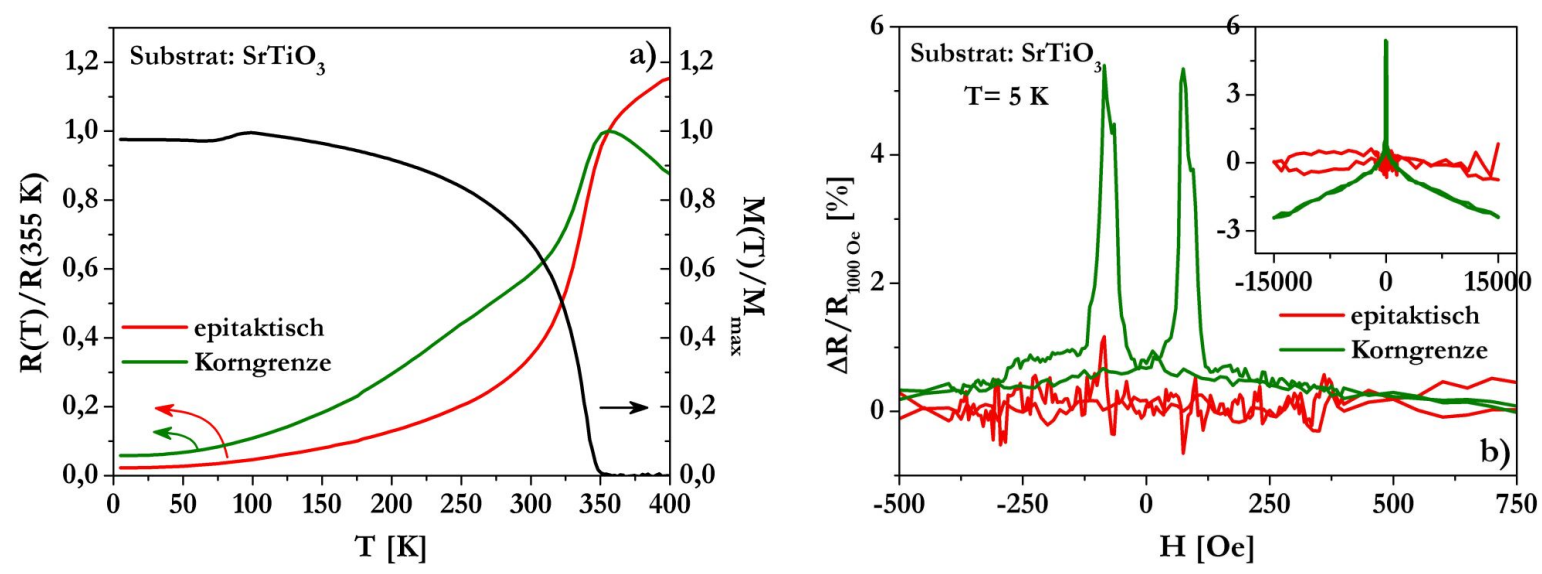

Abbildung 7.5. Magnetotransport einer LSMO-Probe auf einem STO-Bikristall Substrat (Struktur 1). a) Temperaturabhängigkeit des normierten Widerstandes gemessen sowohl ohne als auch mit Berücksichtigung der Korngrenze im Vergleich mit der normierten Magnetisierung. b) Magnetowiderstand bei $T=5 \mathrm{~K}$.

des, oberhalb $T_{C}$ wird der Verlauf wieder flacher. Gegenüber diesem Verhalten zeigen sich Änderungen, wenn die Widerstandsbestimmung die Korngrenze beinhaltet. Oberhalb von $T_{C}$ ergibt sich ein isolierendes Verhalten (im Sinne von $d R / d T<0$ ). Des Weiteren weist der Verlauf eine Schulter im Bereich von ca. $250 \mathrm{~K}$ auf, welche in der Messung auf dem epitaktischen Teil der Probe nicht vorhanden ist.

Ebenso wie für die Temperaturabhängigkeit des Widerstands kann auch für die Magnetfeldabhängigkeit der Einfluss der Korngrenze durch den Vergleich mit der Messungen auf dem epitaktische Teil der Probe gewonnen werden. Dabei werden alle Magnetowiderstandsmessungen über die Korngrenze so durchgeführt, dass das Magnetfeld in der Schichtebene und parallel zur Korngrenze liegt. Der Strom verläuft somit senkrecht zum Magnetfeld.

Der Magnetowiderstand (s. Gl. 6.1) bei $T=5 \mathrm{~K}$, bestimmt mit $I=7 \mu \mathrm{A}$, ist in Abbildung 7.5 b) für beide Messgeometrien dargestellt, es zeigen sich deutliche Unterschiede im Verlauf. Der auf einem Halbkristall bestimmte Widerstand zeigt im Rahmen der Streuung keinerlei Abhängigkeit vom äußeren Magnetfeld. Dies gilt auch für höhere Felder bis zu $15 \mathrm{kOe}$ (siehe Einsatz). Der Magnetowiderstand unter Berücksichtigung der Korngrenze hingegen weist eine deutliche Magnetfeldabhängigkeit auf. Es ergibt sich ein maximaler LFMR von ungefähr 5,5\% bei \pm 80 Oe. Die Widerstandsänderungen sind dabei vergleichsweise scharf und der LFMR damit auf einen kleinen Magnetfeldbereich beschränkt. Ein Vergleich mit Magnetisierungs-Hysteresen ergibt, dass die Koerzitivfelder der Magnetisierungskurven bei gleichen Temperaturen deutlich kleiner sind $\left(H_{C} \approx 40 \mathrm{Oe}\right)$. Während der Magnetisierungsmessungen liegt das äußere Feld aber messtechnisch begründet senkrecht zur Korngrenze, während es zur Bestimmung des LFMR parallel dazu liegt. Auf die Temperaturabhängigkeit des LFMRs wird später eingegangen [s. Einsatz von Abbildung 7.8 b)]. Auch im Hochfeldbereich weist der Widerstand eine Magnetfeldabhängigkeit auf. Es zeigt sich eine hysteresefreie, nahezu lineare Abnahme ohne Hinweise auf eine Sättigung in Feldern bis zu $H=15 \mathrm{kOe}$. 

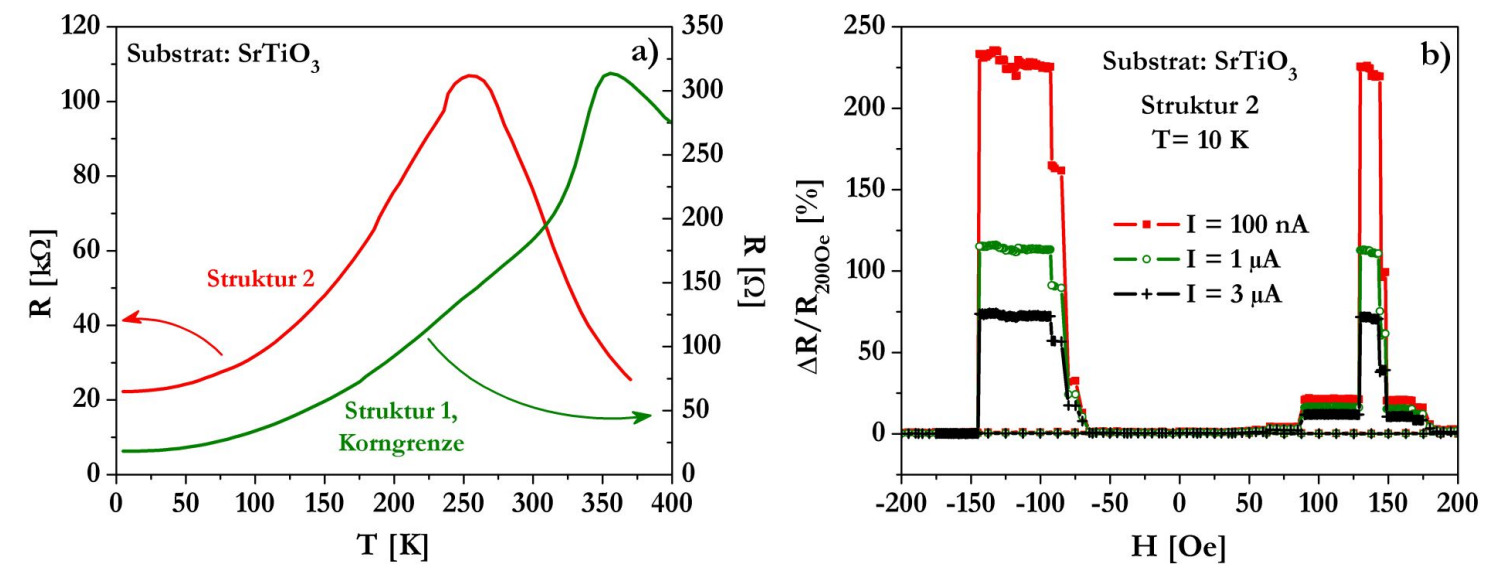

Abbildung 7.6. Magnetotransport einer LSMO-Probe auf einem STO-Bikristall Substrat. a) Vergleich der Temperaturabhängigkeit des Widerstandes über die Korngrenze hinweg von Struktur 1 und 2. b) Magnetowiderstand der Struktur 2 bei $T=10 \mathrm{~K}$ bei verschiedenen Strömen.

Um den Einfluss des epitaktischen Teils in den Messungen über die Korngrenze hinweg zu minimieren und um einen möglichst homogenen Bereich der Korngrenze in der Messung zu analysieren, wird die Struktur 2 mit einer Stegbreite von $d \approx 3 \mu \mathrm{m}$ auf der Probe definiert (s. Kap. 5.5.1). Abbildung 7.6 a) zeigt den temperaturabhängigen Widerstand, gemessen mit $I=1 \mu \mathrm{A}$, im Vergleich mit dem Widerstand der Struktur 1 über die Korngrenze hinweg [vgl. Abbildung 7.5a)]. Im Widerstandsverlauf der Struktur 2 tritt bei $T \approx 250 \mathrm{~K}$, also weit unterhalb $T_{C}$, ein Maximum auf. Bei dieser Temperatur weist der Widerstand der Struktur 1 eine Schulter auf. Im isolierenden Bereich der Struktur 2 zeigt sich bei $T \approx T_{C}$ eine leichte Änderung in der Steigung des Widerstandes. Der minimale Widerstand bei tiefen Temperaturen unterscheidet sich ebenfalls für die beiden Strukturen: Für die Struktur 1 beträgt das Verhältnis $R_{\min , 1} / R_{\max , 1} \approx 0,06$, für die Struktur 2 beträgt es $R_{\min , 2} / R_{\max , 2} \approx 0,2$. Weiterhin sind die Absolutwerte des Widerstandes zu beachten. Für $T=5 \mathrm{~K}$ liegt der Widerstand der Struktur 1 bei $R \approx 20 \Omega$, während er für die Struktur 2 ca. $20 \mathrm{k} \Omega$ beträgt. Es ergibt sich ein Unterschied von drei Größenordnungen. Unter der Annahme eines gleichen spezifischen Widerstandes sollten sich die Widerstände aufgrund der geänderten Geometrie aber nur um einen Faktor von ungefähr vier unterscheiden.

Der auf $H=200$ Oe normierte Magnetowiderstand der Struktur 2, bestimmt bei $T=10 \mathrm{~K}$ mit verschiedenen Stromstärken, ist in Abbildung $7.6 \mathrm{~b}$ ) dargestellt. Es zeigt sich ein deutlich geändertes Verhalten gegenüber der Magnetowiderstandsmessung an Struktur 1. Der Widerstand weist verschiedene stabile Plateaus auf, zwischen denen ein scharfer Übergang stattfindet. Dabei zeigt das Verhalten nicht die zu erwartende Spiegelsymmetrie. Abgesehen von den jeweils höchsten und niedrigsten Widerstandszuständen sind die Plateaus für positive, bzw. negative Felder unterschiedlich. Auch die Breite der Plateaus ist asymmetrisch. So ist der höchste Widerstandszustand für negative Felder in einem Bereich von $\Delta H \approx 50$ Oe stabil, für positive Felder lediglich über $\Delta H \approx 15$ Oe. Auch die Sättigungsfelder unterscheiden sich um ca. 25 Oe. Von besonderer Bedeutung ist der 

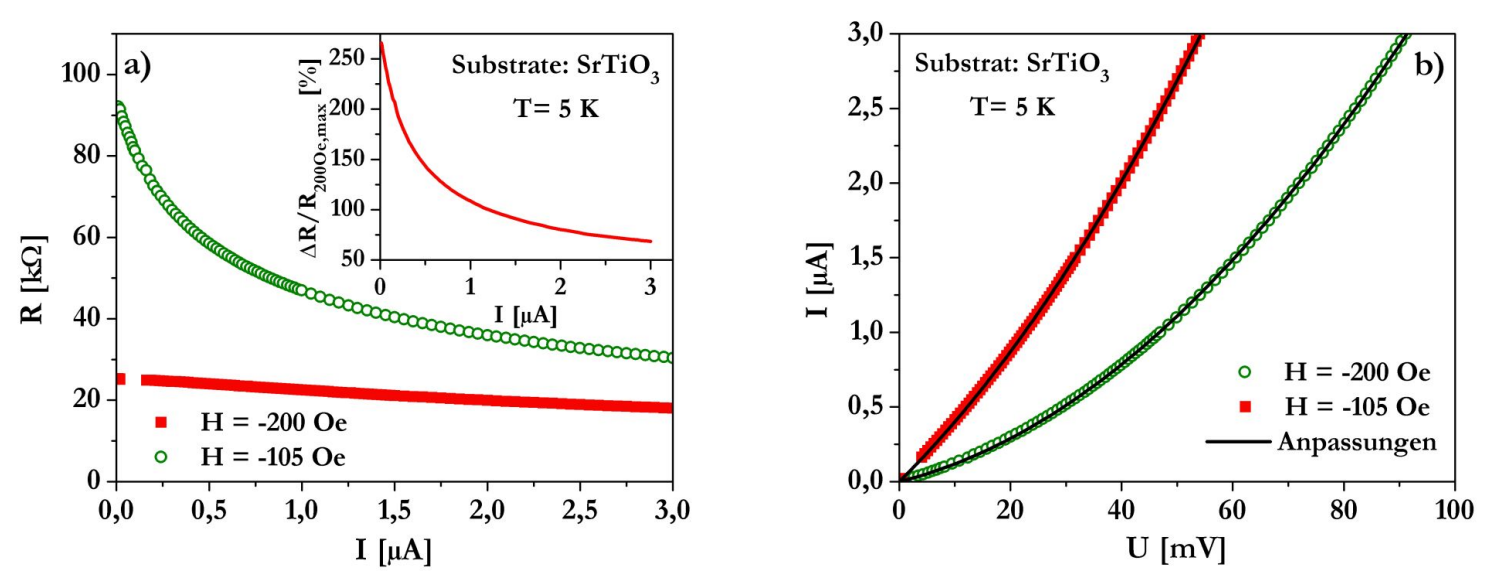

Abbildung 7.7. LSMO-Probe auf STO-Bikristall Substrat. a) Stromabhängigkeit des Widerstandes für unterschiedliche Magnetisierungskonfigurationen bei $T=5 \mathrm{~K}$. Einsatz: Aus den Daten nach a) berechneter Magnetowiderstands-Effekt als Funktion des Stroms. b) Strom-Spannungs-Kennlinien berechnet aus den Daten nach a) für die beiden Magnetisierungskonfigurationen sowie Anpassung nach Glazman-Matveev [121].

hohe Wert des Magnetowiderstandes. Unter Verwendung der Struktur 1 beträgt dieser ca. $5,5 \%$, die Verringerung der Kontaktbreite führt zu einer drastischen Zunahme auf über $230 \%$. Dabei ist der Magnetowiderstand für die Struktur 2 stark stromabhängig. Beträgt er noch bei $10 \mathrm{~K}$ für $I=100 \mathrm{nA}$ ca. $237 \%$, so fällt er für $I=1 \mu \mathrm{A}$ auf $115 \%$ und für $I=3 \mu \mathrm{A}$ auf $75 \%$ ab.

Die detaillierte Stromabhängigkeit des Widerstandes bei $T=5 \mathrm{~K}$ ist in Abbildung 7.7 a) gegeben. Dabei wird das äußere Feld mit Hilfe der Magnetowiderstandskurve 7.6 b) so eingestellt, dass sich das System einmal im hochohmigen Zustand und anschließend im niederohmigen Zustand befindet. In beiden Fällen ist eine Abnahme des Widerstandes mit zunehmender Stromstärke zu beobachten, es liegt kein Ohmsches Verhalten vor. Dabei zeigt sich, dass die Abhängigkeit des Widerstandes von der Stromstärke im hochohmigen Zustand deutlich ausgeprägter ist. Dieses gilt insbesondere für $I<1 \mu \mathrm{A}$. Aus den beiden Widerstandskurven kann leicht der Magnetowiderstand als Funktion des Stromes gewonnen werden [Einsatz von Abbildung 7.7a)]. Aufgrund der unterschiedlichen Abhängigkeiten des Widerstandes in den beiden verschiedenen Zuständen kommt es zu einer starken Abnahme des maximalen Magnetowiderstands-Effekts mit zunehmender Stromstärke. Dennoch beträgt der Magnetowiderstand für eine Stromstärke von $I=3 \mu \mathrm{A}$ noch $75 \%$ und liegt damit deutlich über dem der Struktur 1.

In Abbildung 7.7 b) sind die aus Abbildung a) berechneten Strom-Spannung-Kennlinien dargestellt. Es zeigt sich erwartungsgemäß ein nichtlineares Verhalten. Die Beschreibung der Daten mit dem Modell nach Glazman und Matveev [121] gelingt gut (s.a. Kap. 3.2.3, wohingegen das Simmons-Modell die aus den Daten berechnete Leitfähigkeit nicht zufriedenstellend wiedergeben kann. Dies ist möglicherweise auch auf den relativ geringen zugänglichen Spannungsbereich zurückzuführen. Wegen der limitierten Spannung an der Stromquelle des PPMS sind Stromstärken von $I>3 \mu \mathrm{A}$ im hochohmigen Zustand nicht zugänglich. Bei hohen Temperaturen ist aufgrund des ansteigenden Widerstands selbst 


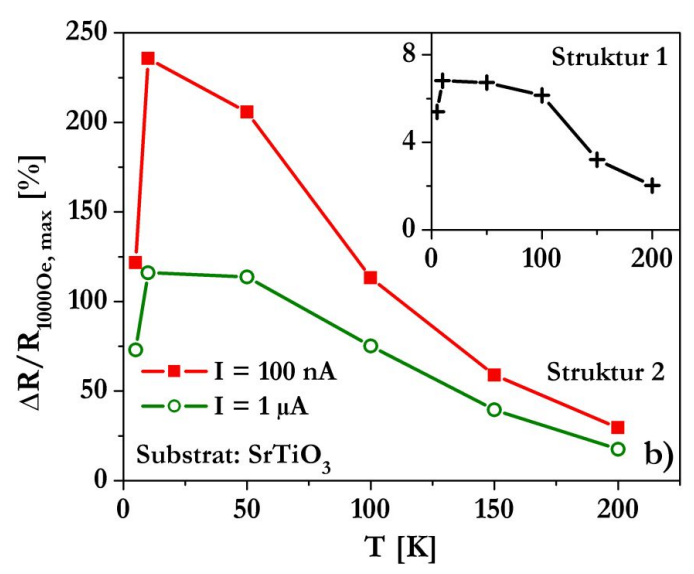

Abbildung 7.8. LSMO-Probe auf STO-Bikristall Substrat: Temperaturabhängigkeit des Magnetowiderstandes der Struktur 2 für $I=100 \mathrm{nA}$ und $I=1 \mu \mathrm{A}$. Einsatz: Temperaturabhängigkeit des Magnetowiderstandes der Struktur 1.

für $I=3 \mu \mathrm{A}$ keine Messung mehr möglich.

Der Wert des maximalen Magnetowiderstandes der Struktur 2, ermittelt aus den Widerstandshysteresen, ist in Abbildung 7.8 als Funktion der Temperatur sowohl für $I=100 \mathrm{nA}$ als auch für $I=1 \mu \mathrm{A}$ aufgetragen. Der Magnetowiderstand weist keine monotone Temperaturabhängigkeit auf. Vielmehr ist der Effekt für $T=10 \mathrm{~K}$ mit $235 \%$ maximal, wohingegen er bei $T=5 \mathrm{~K}$ lediglich $121 \%$ beträgt (bei jeweils $I=100 \mathrm{nA}$ ). Die Temperaturabhängigkeit des LFMR der Struktur 1 ist im Einsatz der Abbildung dargestellt. Der qualitative Verlauf ist ähnlich zu dem der Struktur 2. Der MR beträgt 5,5\% bei $T=5 \mathrm{~K}$ und steigt auf maximale $7 \%$ bei $T=10 \mathrm{~K}$.

\section{LSMO Korngrenzen-Kontakte auf $\mathrm{MgO}$}

Im Folgenden werden die Ergebnisse für eine LSMO-Schicht, deponiert auf einem MgOBikristall, zusammengefasst vorgestellt. Zur Bestimmung des Widerstandes über die Korngrenze hinweg werden sowohl Struktur 1 (breit) als auch Struktur 2 (schmal) mit einer Kontaktbreite von $d=5 \mu \mathrm{m}$ auf der Probe definiert. Auf die Transporteigenschaften ohne Berücksichtigung der Korngrenze wird nicht eingegangen. Abbildung 7.9 a) vergleicht den normierten Widerstand der Strukturen $1(I=7 \mu \mathrm{A})$ und $2(I=1 \mu \mathrm{A})$ mit der normierten Magnetisierung ( $H=100$ Oe). Sowohl die Widerstandskurve der Struktur 1 als auch die Magnetisierungskurve zeigen einen deutlich flacheren Verlauf als die auf STO deponierte Probe (vgl. Abb. 7.5). Dabei ist eine Schulter im Widerstandsverlauf, wie sie im oben vorgestellten System zu beobachten ist, nicht auszumachen. Die magnetische Phasenübergangstemperatur ergibt sich zu $T_{C} \approx 305 \mathrm{~K}$ und stimmt etwa mit der Temperatur maximalen Widerstandes der Struktur 1 überein. Der normierte Widerstand der Struktur 2 liegt für $T \leq T_{C}$ immer unterhalb des Verlaufes von Struktur 1 und zeigt ein gegensätzliches Verhalten zur LSMO-Schicht auf STO. Der Widerstandsanstieg um $T_{C}$ ist für die schmale Struktur deutlich ausgeprägter als für die breite Struktur. Die Temperatur maximalen Widerstandes ist leicht zu höheren Temperaturen verschoben. 

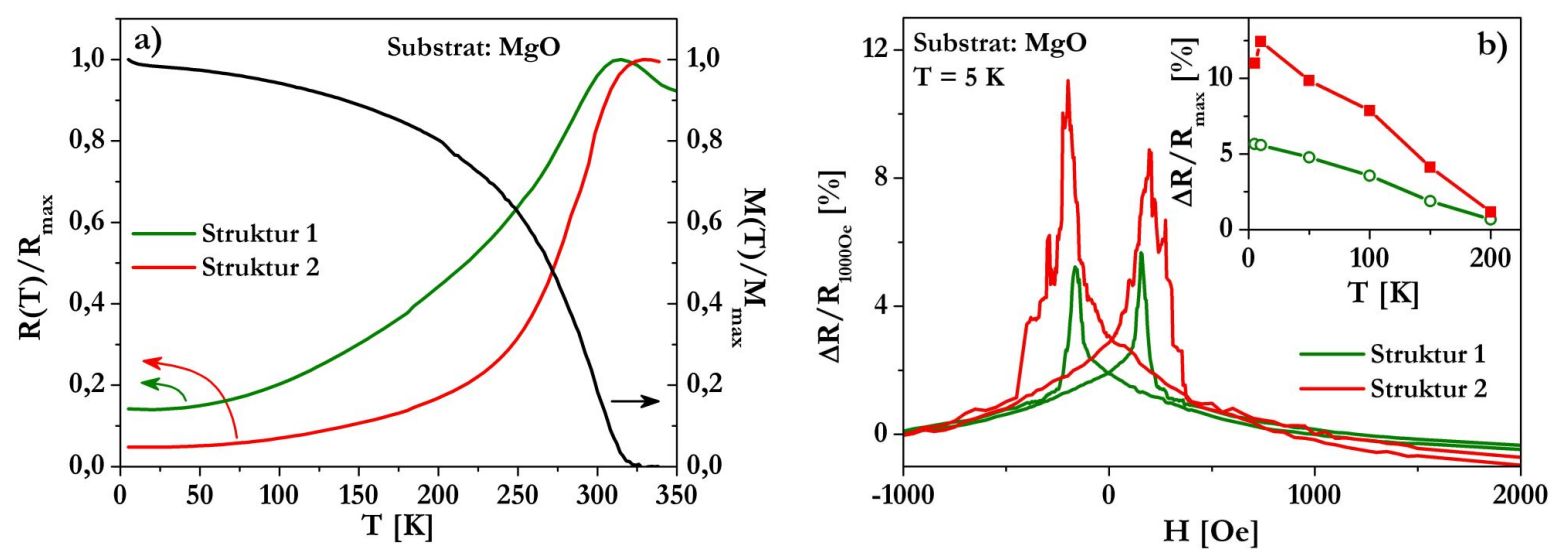

Abbildung 7.9. LSMO-Probe auf MgO-Bikristall Substrat. a) Temperaturabhängigkeit der normierten Magnetisierung, aufgenommen mit $H=100$ Oe, sowie der normierten Widerstände für die Strukturen $1(I=7 \mu \mathrm{A})$ und $2(I=1 \mu \mathrm{A})$. b) Magnetowiderstandsverhalten der Strukturen $1(I=7 \mu \mathrm{A})$ und $2(I=1 \mu \mathrm{A})$ bei $T=5 \mathrm{~K}$. Einsatz: Maximaler LFMF der Strukturen 1 und 2 als Funktion der Temperatur.

Der Absolutwert der schmalen Struktur bei $T=5 \mathrm{~K}$ beträgt ca. $1 \mathrm{k} \Omega$ und ist damit um einen Faktor $\approx 7$ größer als der Absolutwert der breiten Struktur bei $T=5 \mathrm{~K}(R \approx 150 \Omega)$. Aufgrund der unterschiedlichen Geometrien ist ein Unterschied von ungefähr Faktor 2,5 zu erwarten.

Der Magnetowiderstand der Strukturen 1 und 2 bei $T=5 \mathrm{~K}$ ist in Abbildung $7.9 \mathrm{~b}$ ) dargestellt. Es ergeben sich vergleichbare Maximalwerte von 5\% (breite Struktur), bzw. 11\% (schmale Struktur). Auch der qualitative Verlauf ist ähnlich, wobei die Struktur 1 aber einen deutlich glatteren Verlauf aufweist. Für die schmale Struktur ergibt sich für $I \leq 5 \mu \mathrm{A}$ keine Stromabhängigkeit des LFMR. Die breite Struktur zeigt lineare StromSpannungs-Kennlinien für $I \leq 25 \mu \mathrm{A}$, s.d. auch hier keine Stromabhängigkeit erwartet werden kann.

Der Einsatz der Abbildung 7.9 b) stellt die Temperaturabhängigkeit des maximalen LFMR für beide Strukturen dar. Die breite Struktur zeigt wie erwartet ein mit der Temperatur monoton abfallendes Verhalten. Für die schmale Struktur hingegen ist wie für die LSMO-Schicht auf STO der LFMR für $T=10 \mathrm{~K}$ maximal und zeigt erst für höhere Temperaturen ein monotones Verhalten.

\subsubsection{LSMO:MgO Korngrenzen-Kontakte}

Neben den reinen LSMO Bikristallkontakten sind in Analogie zu den Nanokompositen die MgO-dotierten Korngrenzen-Kontakte von besonderem Interesse. Aufgrund der chemischen Phasenseparation und den Erfahrungen mit den Nanokompositen ist zu erwarten, dass das isolierende Material in der künstlichen Korngrenze segregiert und so die Tunnelbarriere verändert.

$(\mathrm{LSMO})_{1-x}:(\mathrm{MgO})_{x}$-Schichten mit $x=0,05$ und $x=0,1$ werden auf STO- und MgOBikristall Substraten deponiert. Die Ergebnisse werden exemplarisch für eine auf STO 

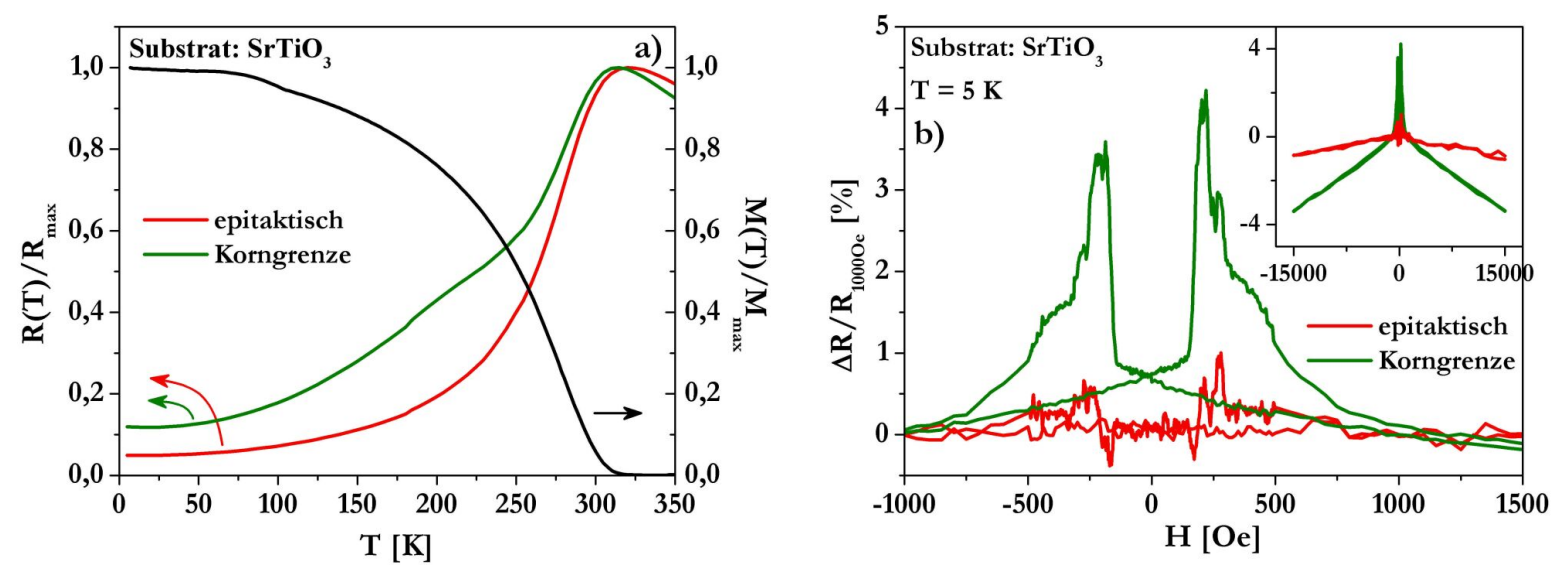

\begin{abstract}
Abbildung 7.10. Magnetotransport einer (LSMO) $)_{0,9}:(\mathrm{MgO})_{0,1}$-Probe auf einem STOBikristall Substrat (Struktur 1). a) Temperaturabhängigkeit des normierten Widerstandes gemessen sowohl ohne als auch mit Berücksichtigung der Korngrenze im Vergleich mit der normierten Magnetisierung. b) Magnetowiderstand bei $T=5 \mathrm{~K}$ und $I=7 \mu \mathrm{A}$.
\end{abstract}

präparierte $(\mathrm{LSMO})_{0,9}:(\mathrm{MgO})_{0,1}$-Schicht detailliert vorgestellt, anschließend folgt eine $\mathrm{Zu}$ sammenfassung einer auf MgO deponierten Schicht gleicher Zusammensetzung.

Zur Bestimmung der Transporteigenschaften wird zunächst Struktur 1 auf der Probe definiert. Abbildung 7.10 a) stellt den daraus gewonnenen normierten Widerstand $(I=7 \mu \mathrm{A})$ als Funktion der Temperatur im Vergleich mit der normierten Magnetisierung $\left(H=100\right.$ Oe) dar. Aus der Magnetisierung wird die Curie-Temperatur von $T_{C}=310 \mathrm{~K}$ gewonnen. Dabei ist auffällig, dass die Magnetisierung einen deutlich flacheren Verlauf zeigt als für die reine Probe. Das Widerstandsverhalten hingegen ist dem der reinen Probe ähnlich: Im Unterschied zur epitaktischen Messung zeigt sich in der Messung über die Korngrenze eine Schulter im Bereich 200-250 K. Die Temperaturen maximalen Widerstandes liegen nahe der Curie-Temperatur und betragen $T\left(R_{\max }\right) \approx 315 \mathrm{~K}$ für die Messung über die Korngrenze, bzw. $T\left(R_{\max }\right) \approx 320 \mathrm{~K}$ für die Messung auf dem epitaktischen Teil der Probe. Für beide Messungen gilt dabei oberhalb dieser Temperatur $d R / d T<0$.

Das Magnetowiderstandsverhalten bei $T=5 \mathrm{~K}$ und $I=7 \mu \mathrm{A}$ ist in Abbildung $7.10 \mathrm{~b}$ ) aufgetragen. Es zeigt sich ein deutlicher LFMR in der Messung über die Korngrenze, wohingegen in der Messung auf dem epitaktischen Teil nur kleine Effekte auftreten. Von hohen, positiven Feldern kommend, weist die Messung des epitaktischen Teils bei kleinen negativen Feldern zunächst ein Minimum und anschließend ein schwaches Maximum im Magnetowiderstand auf. Dieses Verhalten zeigt sich auch im positiven Feldbereich, wenn das Feld aus negativer Sättigung erhöht wird. Zusätzlich wird eine schwache, nichthysteretische, annähernd lineare Abnahme des Widerstandes für hohe Felder beobachtet [s. Einsatz von Abbildung $7.10 \mathrm{~b}$ )]. Bis zu $15 \mathrm{kOe}$ wird keine Sättigung erreicht. Diese lineare Abnahme tritt auch für die Messung über die Korngrenze auf, wo sie aber deutlich ausgeprägter ist. Bei $15 \mathrm{kOe}$ beträgt sie ca. $-4 \%$, es wird ebenfalls keine Sättigung erreicht. Der Wert des LFMR über die Korngrenze ist vergleichbar mit denen der reinen Proben für die Struktur 1. Bei $T=5 \mathrm{~K}$ beträgt er etwas über $4 \%$, wobei aber die Form der Widerstandshysterese deutlich geändert ist. Wiederum von hohen, positiven Feldern 

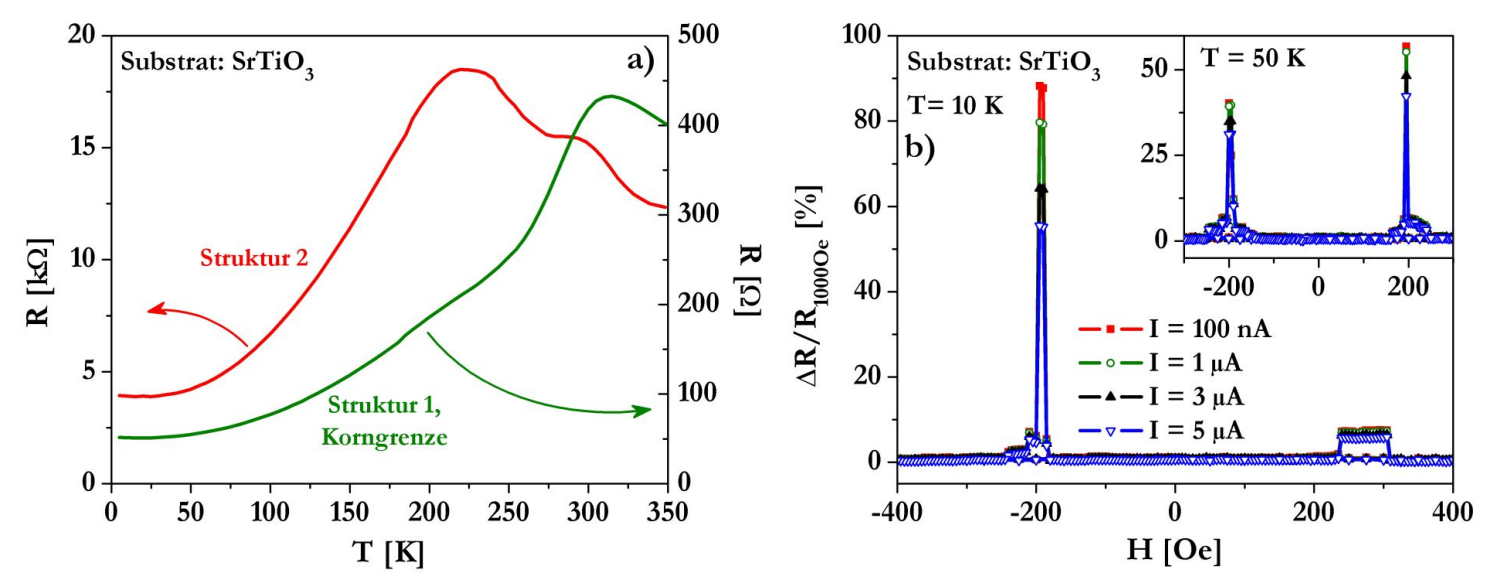

Abbildung 7.11. Magnetotransport einer (LSMO) $)_{0,9}:(\mathrm{MgO})_{0,1}-$ Probe auf einem STOBikristall Substrat. a) Vergleich der Temperaturabhängigkeit des Widerstandes über die Korngrenze hinweg von Struktur 1 und 2. b) Magnetowiderstand der Struktur 2 für $T=10 \mathrm{~K}$ bei verschiedenen Strömen. Einsatz: Selbiges für $T=50 \mathrm{~K}$.

kommend zeigt der Widerstand bei ca. -160 Oe einen Anstieg des Magnetowiderstandes auf annähernd den Maximalwert. Eine weitere Erhöhung des Feldes in Richtung negativer Werte führt zu einer graduellen Abnahme des MRs bis zur Sättigung bei ca. -850 Oe. Dieses Verhalten ist dem der reinen LSMO-Schicht auf einem MgO-Substrat entgegengesetzt: Dort findet für die Messung der Struktur 1 über die Korngrenze zunächst ein gradueller Anstieg statt, bevor der LFMR annähernd sprunghaft in die Sättigung geht [s. Abb. 7.9 a)].

Zur weiteren Untersuchung der Korngrenze wird die Struktur 2 mit einer Breite von $d \approx 2,5 \mu \mathrm{m}$ auf der Probe definiert. Der Vergleich der Temperaturabhängigkeit des Widerstandes, gemessen über die Korngrenze unter Verwendung der beiden verschiedenen Mikrostrukturen, ist in Abbildung 7.11 a) gegeben. Die Absolutwerte der Widerstände unterscheiden sich deutlich. Bei $T=5 \mathrm{~K}$ beträgt er ca. $50 \Omega$ für die breite und ca. $4 \mathrm{k} \Omega$ für die schmale Struktur. Aufgrund der Geometrie wird ein Unterschied um Faktor 4,5 erwartet, beobachtet wird ein Unterschied um Faktor 80. Die in der Struktur 1 beobachtete Schulter im Temperaturbereich 200-250 K wird für die schmale Struktur dominant: Der Widerstand ist bei ca. $220 \mathrm{~K}$ maximal. In der Nähe von $T_{C}$ wird eine Schulter im Widerstandsverlauf beobachtet.

Das Magnetowiderstandsverhalten der Struktur 2 bei $T=10 \mathrm{~K}$ ist für verschiedene Stromstärken in Abbildung 7.11 b) dargestellt. Es zeigt sich ein deutlich geändertes Verhalten gegenüber der Struktur 1: Es treten nur sehr scharfe, definierte Widerstandsänderungen auf, welche auf einen engen Feldbereich begrenzt sind. Dabei sind die Felder der größten Widerstandsänderungen höher als für die Struktur 1. Weiterhin ist das Verhalten stark asymmetrisch. Für negative Felder wird ein Magnetowiderstands-Effekt von bis zu 90\% gemessen, für positive Felder lediglich 7\%. Auch die Felder, bei denen die Widerstandsänderungen auftreten, unterscheiden sich für die beiden Feldpolaritäten. Die bei $T=10 \mathrm{~K}$ auftretende Asymmetrie verschwindet weitgehend bei $T=50 \mathrm{~K}$. Wie im Einsatz von Abbildung 7.11 b) dargestellt, weist der Magnetowiderstand hohe Werte 

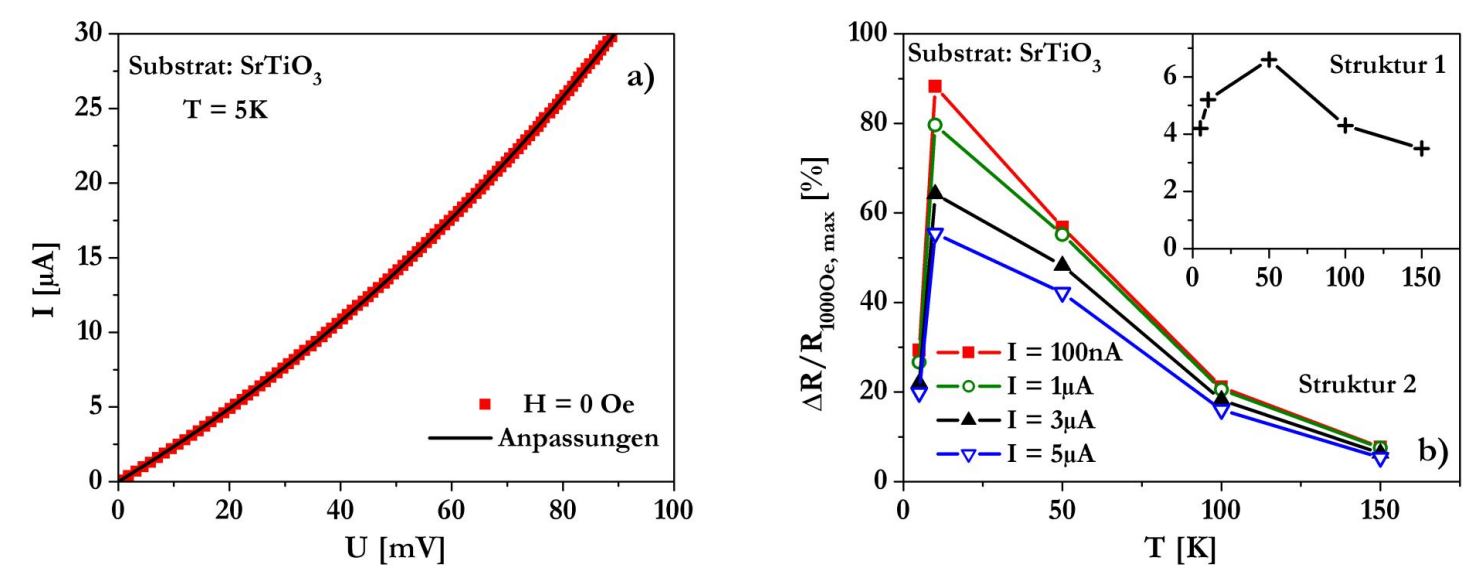

\begin{abstract}
Abbildung 7.12. (LSMO $)_{0,9}:(\mathrm{MgO})_{0,1}$-Probe auf STO-Bikristall Substrat: a) StromSpannungs-Kennlinie der Struktur 2 bei $T=5 \mathrm{~K}$ und $H=0$ Oe sowie Anpassung nach Glazman-Matveev [121. b) Temperaturabhängigkeit der maximalen LFMR der Struktur 2 für verschiedene Ströme. Einsatz: Selbiges für Struktur 1.
\end{abstract}

für beide Feldrichtungen auf, wobei auch die Feldwerte symmetrisch um die Null liegen. Der maximale Magnetowiderstand ist stark stromabhängig. Für $T=10 \mathrm{~K}$ beträgt er ca. $90 \%$ bei $I=100 \mathrm{nA}$ und sinkt sukzessive auf ca. $55 \%$ bei $I=5 \mu$ A. Dabei ist der Einfluss der Stromstärke auf den LFMR für die Maximalwerte deutlich größer als auf stabile Zwischenzustände. Dieses Verhalten wird auch bei anderen Temperaturen (s. Einsatz) beobachtet. Die Strom-Spannungs-Kennlinie des schmalen Kontaktes, gemessen in Stromkontrolle, ist für $T=5 \mathrm{~K}$ und $H=0$ Oe in Abbildung 7.12 a) dargestellt. Die maximale Spannung ist dabei wiederum messtechnisch auf $95 \mathrm{mV}$ begrenzt. Dennoch zeigt sich auch in diesem kleinen Spannungsbereich ein nichtlinearer Zusammenhang. Wie bereits für die reine Schicht auf STO (s. Abb. 7.7 b) können die Daten gut durch das GlazmanMatveev-Modell 121 beschrieben werden, was wiederum nicht für die Beschreibung der aus der Kennlinie berechneten Leitfähigkeit mit dem Simmons-Modells $|98|$ gilt. Eine Strom-Spannungs-Kennlinie in einer hochohmigen Magnetisierungskonfiguration ist nicht zugänglich, s.d. eine direkte Bestimmung der Stromabhängigkeit des LFMR nicht möglich ist.

Die Temperaturabhängigkeit des LFMR der Struktur 2 ist für verschiedene Stromstärken in Abbildung 7.12 b) aufgetragen. Der LFMR ist maximal für $T=10 \mathrm{~K}$ und weist kein monotones Temperaturverhalten auf. Mit höheren Stromstärken nimmt der Magnetowiderstand ab. Dabei ist diese Abnahme bei hohen Magnetowiderstandswerten besonders ausgeprägt. Das nichtmonotone Temperaturverhalten des LFMR gilt auch für Struktur 1, wie der Einsatz der Abbildung darstellt. Hier steigt der LFMR von tiefen Temperaturen bis $T=50 \mathrm{~K}$ an und fällt erst dann mit zunehmender Temperatur. Die Absolutwerte des LFMR sind jedoch bedeutend geringer als für Struktur 2. Für die breite Struktur beträgt er maximal 7\%, für die schmale Struktur hingegen über $85 \%$. 

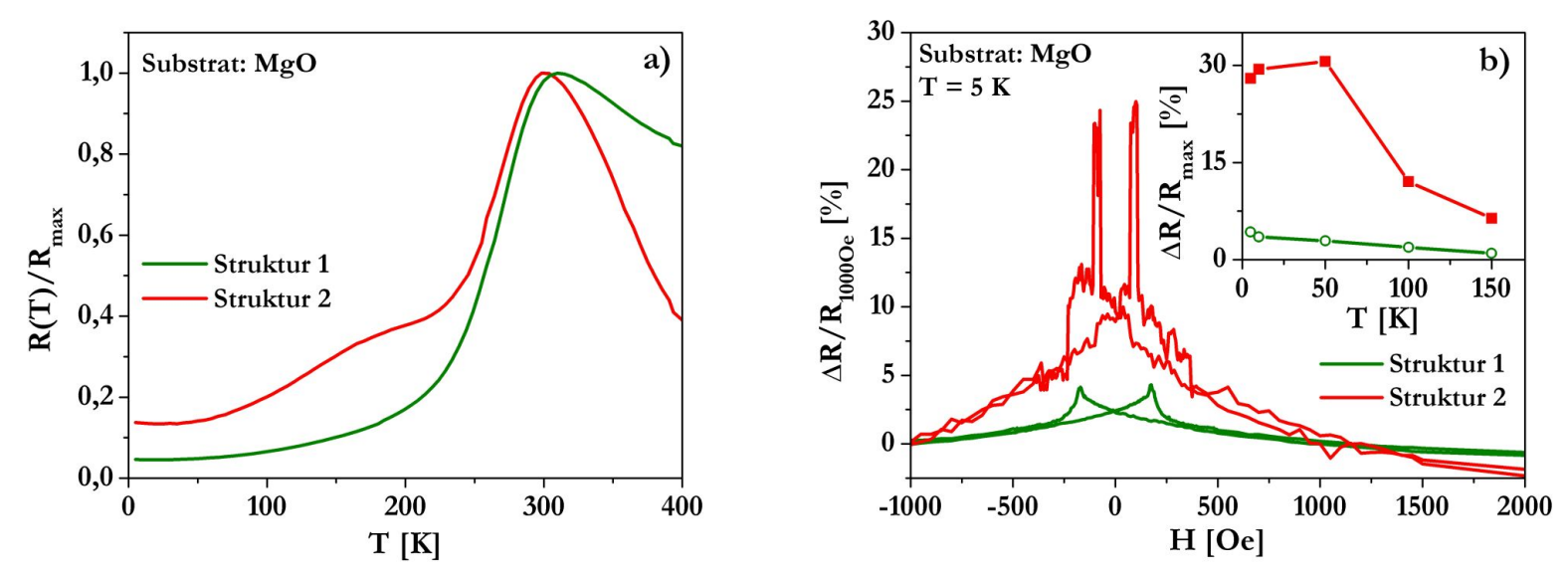

Abbildung 7.13. (LSMO) $)_{0,9}:(\mathrm{MgO})_{0,1}$-Probe auf MgO-Bikristall Substrat. a) Temperaturabhängigkeit des normierten Widerstandes für die Strukturen $1(I=7 \mu \mathrm{A})$ und $2(I=1 \mu \mathrm{A})$. b) Magnetowiderstandsverhalten der Strukturen $1(I=7 \mu \mathrm{A})$ und $2(I=1 \mu \mathrm{A})$ bei $T=5 \mathrm{~K}$. Einsatz: Maximaler LFMR der Strukturen 1 und $2(I=100 \mathrm{nA})$ als Funktion der Temperatur.

\section{LSMO:MgO Korngrenzen-Kontakte auf $\mathrm{MgO}$}

Im Folgenden werden die Ergebnisse einer Schicht identischer Zusammensetzung und Schichtdicke vorgestellt, welche jedoch auf einem MgO-Substrat deponiert wurde. Sowohl Struktur 1 als auch Struktur 2 mit $d \approx 4 \mu \mathrm{m}$ werden auf der Probe definiert. Die Temperaturabhängigkeit des Widerstandes über die Korngrenze für die beiden Strukturen ist in Abbildung 7.13 a) dargestellt. Für $T \leq 200 \mathrm{~K}$ steigt der Widerstand der breiten Struktur mit zunehmender Temperatur langsam an, für höhere Temperaturen hingegen wird der Anstieg stärker. Der Widerstand erreicht bei $T \approx 310 \mathrm{~K}$ ein Maximum, d.h. für höhere Temperaturen gilt $d R / d T<0$. Die schmale Struktur weist ein ähnliches Verhalten auf, zwei Unterschiede sind aber immanent. Einerseits zeigt sich wiederum eine Schulter im Temperaturbereich 150-200 K, andererseits fällt der Widerstand im Hochtemperaturbereich bedeutend stärker ab als für die breite Struktur. Dabei ist die Temperatur maximalen Widerstandes nur geringfügig kleiner als für Struktur 1 (305 K). Ein Vergleich der Absolutwerte der Widerstände bei $T=5 \mathrm{~K}$ führt zu einem Unterschied von Faktor 160 und somit zu einer beträchtlichen Abweichung vom geometrisch zu erwartenden Wert von Faktor 4.

Der Vergleich der Magnetowiderstandskurven der beiden Strukturen ist in Abbildung $7.13 \mathrm{~b}$ ) für $T=5 \mathrm{~K}$ gegeben. Die breite Struktur weist nur einen geringen LFMR von knapp über $4 \%$ auf. Der LFMR dieser Struktur erhöht sich signifikant, wenn der Winkel zwischen Korngrenze und Magnetfeld von $0^{\circ}$ auf $30^{\circ}$ geändert wird: Bei $T=5 \mathrm{~K}$ ergibt sich beispielsweise ein Wert von fast 7\%. Die Form der Widerstandshysterese ist der der reinen LSMO-Probe auf einem MgO-Substrat unter Verwendung der gleichen Mikrostruktur ähnlich [s. Abb. 7.9b)]. Die schmale Mikrostruktur hingegen zeigt einen sichtlich erhöhten LFMR. Im gesamten Feldbereich lässt sich ein annähernd linearer Zusammenhang von Widerstand und Magnetfeld beobachten, dem scharfe Widerstandsänderungen aufgesetzt scheinen. Diese Plateaus machen dabei den größten Teil des LFMR aus und sind auf einen 


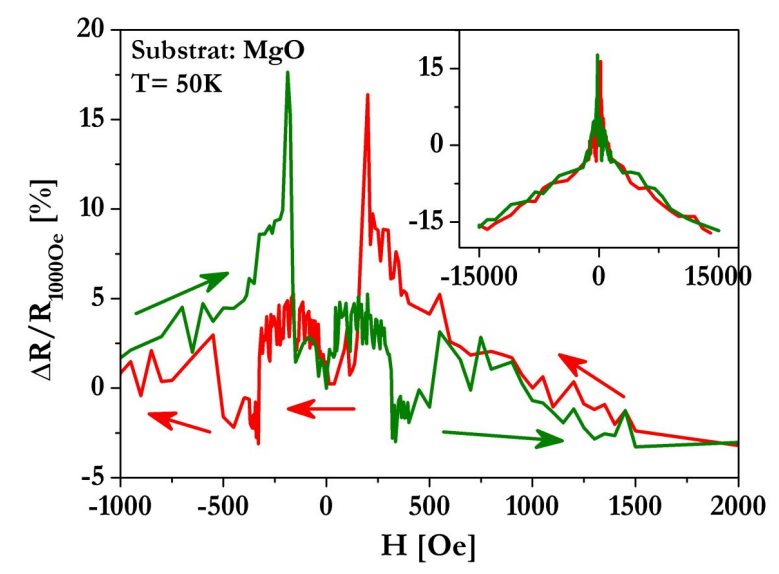

Abbildung 7.14. $(\mathrm{LSMO})_{0,9}:(\mathrm{MgO})_{0,1}$-Probe auf MgO-Bikristall Substrat. Magnetowiderstand der FIB-Struktur für $T=50 \mathrm{~K}$ bei $I=100 \mathrm{nA}$. Rot: $15 \mathrm{kOe} \rightarrow-15 \mathrm{kOe}$; Grün: $15 \mathrm{kOe} \rightarrow 15 \mathrm{kOe}$.

schmalen Feldbereich begrenzt.

Die Temperaturabhängigkeit des maximalen LFMR beider Strukturen ist im Einsatz der Abbildung dargestellt. Für die breite Struktur ergibt sich eine monotone Abnahme, was auch für die Messungen der um $30^{\circ}$ gedrehten Probe gilt. Der LFMR ist insgesamt aber klein und beträgt maximal 4,3\%, bzw. fast $7 \%$ für die gedrehte Probe. Die schmale Stuktur zeigt für $T \leq 50 \mathrm{~K}$ einen nahezu konstanten LFMR mit leicht ansteigender Tendenz. Für höhere Temperaturen ist eine starke Abnahme zu beobachten.

Die Strom-Spannungs-Kennlinien (nicht abgebildet) weisen für die schmale Struktur einen deutlich nichtlinearen Charakter auf. Durch die Bestimmung des Widerstandes für unterschiedliche Magnetisierungskonfigurationen gelingt die direkte Messung der Stromabhängigkeit des LFMR bei $T=5 \mathrm{~K}$. Er fällt kontinuierlich von $33 \%$ bei $I=200 \mathrm{nA}$ auf ca. $15 \%$ bei $I=20 \mu \mathrm{A}$ ab.

Zur weiteren Charakterisierung sowie zur Vorbereitung der Untersuchung strominduzierter Prozesse unter Verwendung hoher Stromdichten wird Struktur 2 an der Korngrenze mit Hilfe des FIBs weiter verjüngt (s. Kap. 5.5.2). Die Breite des FIB-Kontaktes über die Korngrenze beträgt $d \approx 400 \mathrm{~nm}$.

Der Kleinfeld-Magnetowiderstand dieser Struktur, welcher für $T=50 \mathrm{~K}$ in Abbildung 7.14 dargestellt ist, zeigt ein unübliches Verhalten. Von hohen positiven Feldern kommend steigt der Widerstand zunächst kontinuierlich bis zu einem Feld von $H=200 \mathrm{Oe}$ an, wo sich ein scharfes Maximum ergibt. Anschließend geht der Widerstand innerhalb eines sehr schmalen Feldbereiches stark auf ein stabiles Widerstandsniveau zurück. Für negative Felder von ca. -230 Oe wird ein weiterer Rückgang des Widerstandes beobachtet. Es bildet sich erneut ein stabiler Widerstandszustand, der bei ca. -500 Oe verlassen wird. Anschließend fällt der Widerstand kontinuierlich mit steigender Feldstärke ab. Für die umgekehrte Feldrichtung wird das gleiche Verhalten beobachtet. Die kontinuierliche Abnahme des Widerstandes, die für hohe Felder auftritt, ist dabei nichthysteretisch, wie aus dem Einsatz der Abbildung ersichtlich ist. Das vorgestellte Verhalten zeigt sich qualitativ auch bei $T=5 \mathrm{~K}$, der Verlauf ist aber deutlich verrauschter. 


\subsubsection{LSMO: $\mathrm{CeO}_{2}$ Korngrenzen-Kontakte}

In Analogie zu den Nanokompositen wird neben der MgO-Dotierung der Korngrenze auch eine $\mathrm{CeO}_{2}$-Dotierung vorgenommen. Hierzu wird eine $50 \mathrm{~nm}$ dicke $(\mathrm{LSMO})_{0,8}:\left(\mathrm{CeO}_{2}\right)_{0,2}$ -Schicht auf einem STO-Bikristall Substrat präpariert. Zur Untersuchung der Transporteigenschaften werden wie bereits für die anderen Proben sowohl Struktur 1 als auch Struktur 2 mit $d=4 \mu \mathrm{m}$ definiert.

Der normierte Widerstand als Funktion der Temperatur ist für beide Strukturen in Abbildung 7.15a) aufgetragen. Die Messungen von Struktur 1 zeigen so gut wie keine Abweichungen voneinander, die Korngrenze hat offenbar einen sehr geringen Einfluss auf das Widerstandsverhalten. Im Bereich $T \leq 300 \mathrm{~K}$ steigt der Widerstand leicht mit zunehmender Temperatur an, für höhere Temperaturen ist ein stärkerer Anstieg zu beobachten. Im gesamten charakterisierten Temperaturintervall $(5 \mathrm{~K} \leq T \leq 380 \mathrm{~K})$ gilt dabei $d R / d T>0$. Das Widerstandsverhalten von Struktur 2 zeigt einen qualitativ ähnlichen Verlauf. Besonders im Tieftemperaturbereich sind aber Abweichungen zu erkennen. Einerseits zeigt sich die schon oftmals beobachtete Schulter im Bereich 200-250 K, andererseits gilt für $T \leq 30 \mathrm{~K} d R / d T<0$. Weiterhin ist festzustellen, dass die Widerstandsverhältnisse $R_{\max } / R_{\text {min }}$ voneinander abweichen. Für die breite Struktur geht der Widerstand bei tiefen Temperaturen auf unter $2 \%$ des Maximalwertes zurück, für die schmale Mikrostruktur beträgt er im Minimum hingegen ca. $9 \%$ des Maximalwiderstandes. Das Verhältnis der Absolutwerte bei $T=5 \mathrm{~K}$ beträgt $R^{\mathrm{S} 2} / R^{\mathrm{S} 1}=850 \Omega / 34 \Omega=25$ und ist somit gegenüber dem geometrisch zu erwartenden Wert von 4 erhöht.

Die zu den temperaturabhängigen Widerstandsbestimmungen zugehörigen Magnetowiderstandsmessungen bei $T=5 \mathrm{~K}$ sind in Abbildung $7.15 \mathrm{~b}$ ) dargestellt. Ohne Einfluss der Korngrenze zeigt sich im Rahmen der Messgenauigkeit keine Widerstandsänderungen in Feldern bis zu 15 kOe. Unter Einfluss der Korngrenze zeigt die breite Struktur einen typischen Verlauf, wie er beispielsweise für die reine LSMO-Probe auf einem MgO-Substrat für ebenfalls die breite Struktur beobachtet wird (s. Abb. 7.9 b). Allerdings ist die Widerstandshysterese deutlich breiter, der Widerstand ist für ca. \pm 685 Oe maximal und für $H=1000$ Oe nicht vollständig gesättigt. Die Berechnung des LFMR wird deshalb auf einen Wert von $R(3000$ Oe) normiert. Im Hochfeldbereich ergibt sich die bereits bekannte, annähernd lineare Widerstandsabhängigkeit vom äußeren Feld, welche keine Hysterese zeigt.

Das Magnetowiderstandsverhalten ändert sich deutlich bei Verwendung der schmalen Mikrostruktur. Eine exakte Trennung zwischen Kleinfeld- und Hochfeld-Magnetowiderstand ist aufgrund des großen Einflusses des Hochfeldanteils auch bei kleinen Felder nicht durchführbar. Die kontinuierliche Änderung des Widerstandes macht bei Normierung auf $R(3000$ Oe) einen Magnetowiderstands-Effekt von über $12 \%$ aus und übertrifft damit bereits den LFMR der breiten Struktur. Zusätzlich finden sich noch scharfe Widerstandsänderungen mit definierten Widerstandszuständen. Diese treten einerseits als Plateaus bei ca. \pm 300 Oe auf, andererseits zeigen sich scharfe Maxima bei ca. \pm 650 Oe, was ungefähr dem Feldwert maximalen Widerstandes von Struktur 1 entspricht. Der qualitative Verlauf ist in ungefährer Übereinstimmung mit dem der LSMO:MgO-Probe auf einem 

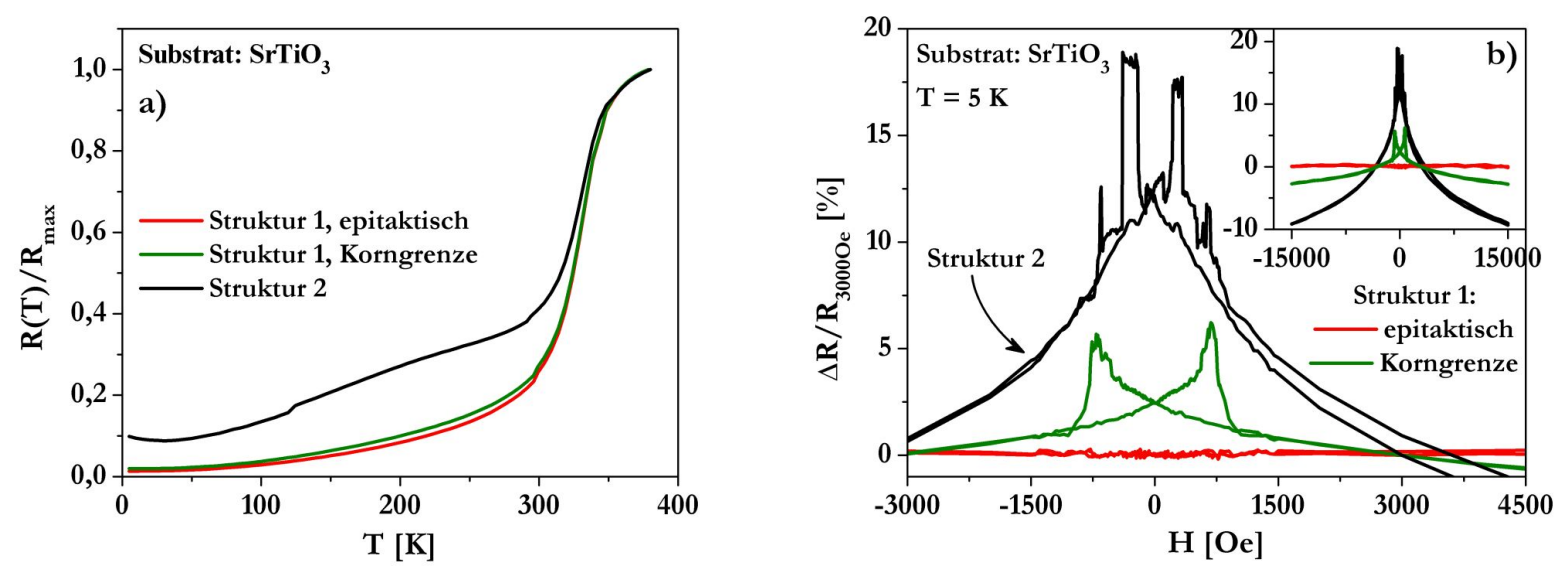

Abbildung 7.15. (LSMO $)_{0,8}:\left(\mathrm{CeO}_{2}\right)_{0,2}$-Probe auf STO-Bikristall Substrat. a) Temperaturabhängigkeit des normierten Widerstandes für die Strukturen $1(I=5 \mu \mathrm{A})$ und $2(I=1 \mu \mathrm{A})$. b) Magnetowiderstandsverhalten der Strukturen 1 und 2 (jeweils $I=1 \mu \mathrm{A}$ ) bei $T=5 \mathrm{~K}$.
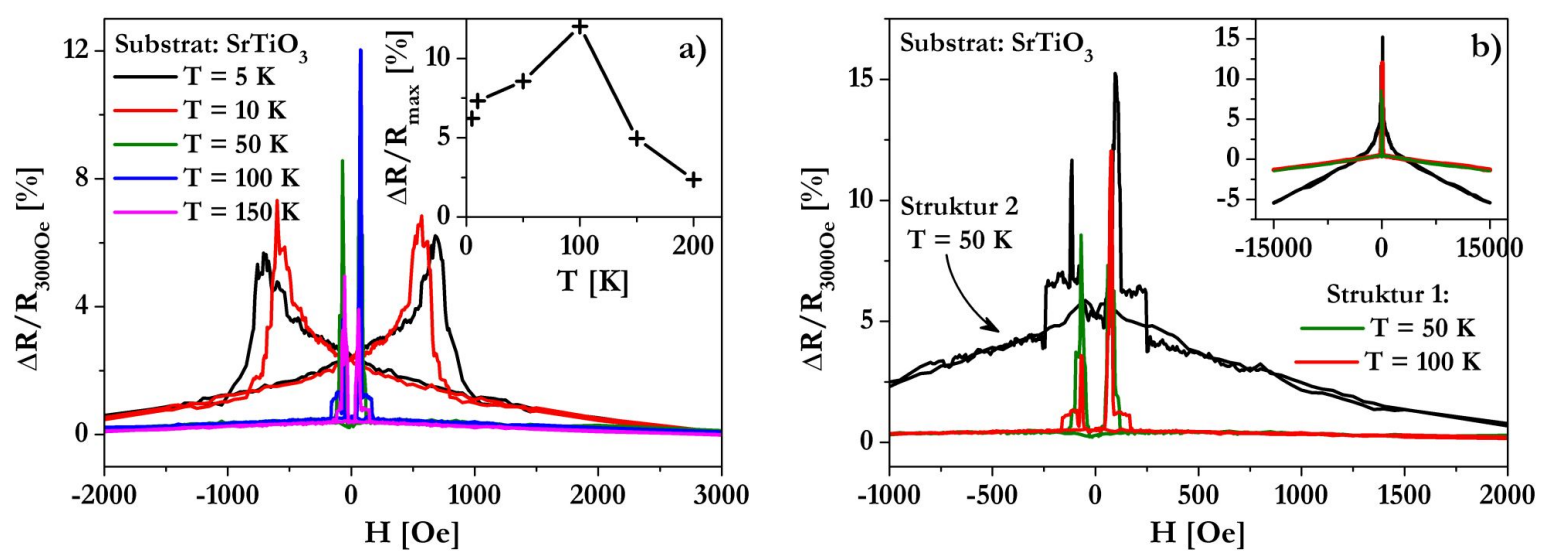

Abbildung 7.16. (LSMO $)_{0,8}:\left(\mathrm{CeO}_{2}\right)_{0,2}$-Probe auf STO-Bikristall Substrat. a) Magnetowiderstandsverhalten der Struktur 1 bei unterschiedlichen Temperaturen $(I=5 \mu \mathrm{A})$. b) Magnetowiderstandsverhalten der Strukturen $1(I=5 \mu \mathrm{A})$ und $2(I=1 \mu \mathrm{A})$ bei $T=50 \mathrm{~K}$.
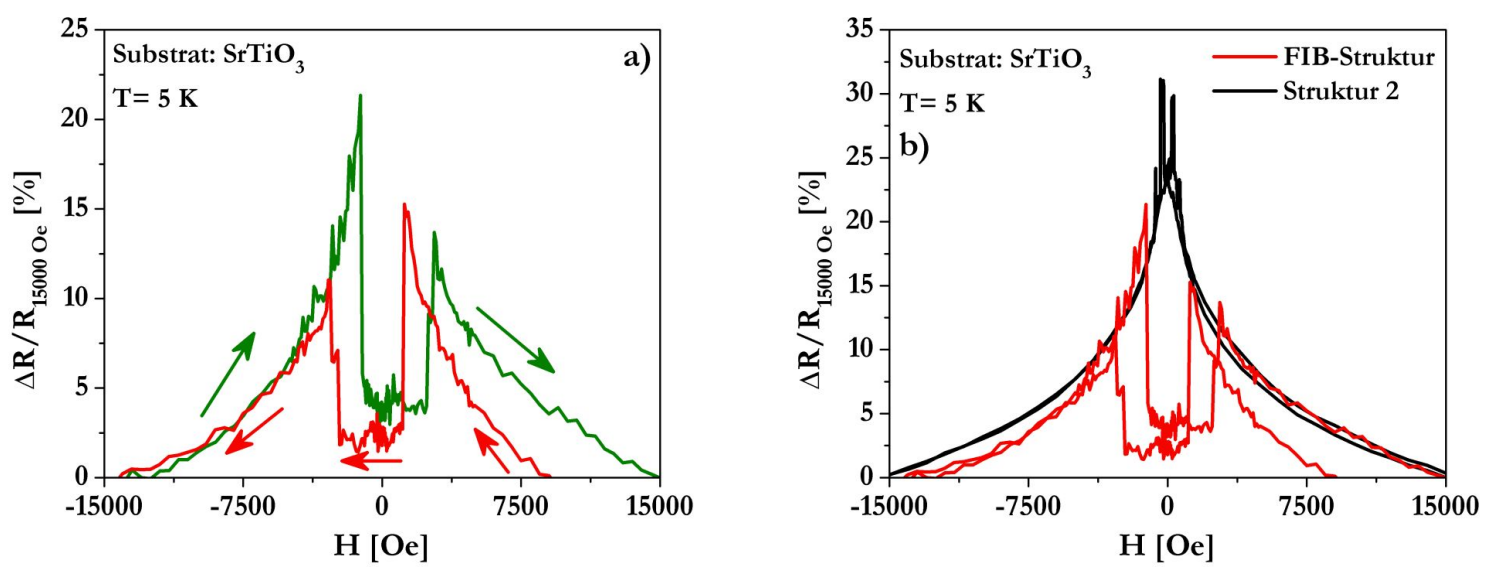

Abbildung 7.17. (LSMO $)_{0,8}:\left(\mathrm{CeO}_{2}\right)_{0,2}$-Probe auf STO-Bikristall Substrat. a) Magnetowiderstand des FIB-Kontaktes bei $T=5 \mathrm{~K}$ und $I=20 \mu \mathrm{A}$. Rot: $15 \mathrm{kOe} \rightarrow-15 \mathrm{kOe}$; Grün: $-15 \mathrm{kOe} \rightarrow 15 \mathrm{kOe}$. 
MgO-Bikristall Substrat unter Verwendung der schmalen Struktur [s. Abb. 7.13b)].

Das Magnetowiderstandsverhalten der breiten Struktur zeigt eine ungewöhnliche Temperaturabhängigkeit. Für alle bisher vorgestellten Magnetowiderstandssysteme gilt, dass sich die Form der Widerstandshysterese durch Variation der Temperatur im Wesentlichen nicht ändert. Für den vorliegenden $\mathrm{CeO}_{2}$-dotierten Korngrenzen-Kontakt ist dieses nicht der Fall. Die Magnetowiderstandskurven, aufgenommen bei unterschiedlichen Temperaturen im Bereich $5 \mathrm{~K} \leq T \leq 150 \mathrm{~K}$, weisen zwei unterschiedliche Verlaufsarten auf, siehe Abbildung 7.16 a). Für $T \leq 10 \mathrm{~K}$ wird eine vergleichsweise harte magnetische Hysterese mit Sättigungsfeldern im Bereich 1000 Oe beobachtet (s.o.). Für $T \geq 50 \mathrm{~K}$ ergibt sich ein vollständig geändertes Verhalten. Die Widerstandsänderungen sind auf einen Bereich $|H| \leq 200$ Oe beschränkt und zeigen scharfe Änderungen mit teilweise wohldefinierten Widerstandsplateaus. Für Felder höher als 200 Oe ändert sich der Widerstand kaum und darüber hinaus im gesamten Feldbereich $H>200$ Oe annähernd linear. Die maximalen Widerstandsänderungen der beiden Typen sind vergleichbar. Im Einsatz der Abbildung 7.16 a) ist der maximale LFMR als Funktion der Temperatur aufgetragen. Der LFMR liegt in einer Größenordnung von $10 \%$ und zeigt für beide Arten von Widerstandsverhalten zunächst einen Anstieg mit zunehmender Temperatur.

Das geänderte Magnetowiderstandsverhalten für $T \geq 50 \mathrm{~K}$ wird in Abbildung $7.16 \mathrm{~b}$ ) mit dem Verhalten des schmalen Kontaktes für $T=50 \mathrm{~K}$ verglichen. Im Kleinfeldbereich offenbart sich ein qualitativ ähnlicher Verlauf. Wird der nichthysteretische MR-Anteil für Struktur 2 zunächst außer Acht gelassen, weisen alle Verläufe scharfe Maxima im Bereich 70-100 Oe auf. Zusätzlich treten in allen Messungen wohldefinierte Widerstandsplateaus auf, welche für Struktur 2 aber deutlich ausgeprägter sind. Im Hochfeldbereich hingegen unterscheiden sich die Verläufe. Für die breite Struktur ändert sich der Widerstand nur wenig, die schmale Struktur zeigt einen hohen MR von mehr als $-5 \%$ bei $H=15 \mathrm{kOe}$.

Auch in diesem Fall wird mittels des FIBs der Korngrenzen-Kontakt von Struktur 2 weiter ausgedünnt. Die Breite des über die Korngrenze präparierten Kontaktes beträgt $d \approx 250 \mathrm{~nm}$. Die Temperaturabhängigkeit des Widerstandes wird beim Abkühlen des Kontaktes im Bereich $60 \mathrm{~K} \leq T \leq 300 \mathrm{~K}$ aufgenommen (nicht abgebildet). Es ergibt sich ein ansteigender Widerstand mit zunehmender Temperatur bis zu $T \approx 250 \mathrm{~K}$, wo sich ein schwaches Maximum ergibt. Für $T \geq 280 \mathrm{~K}$ steigt der Widerstand wieder an.

Der Magnetowiderstand bei $T=5 \mathrm{~K}$, bestimmt mit $I=20 \mu \mathrm{A}$, ist in Abbildung 7.17 a) dargestellt. Dabei wird aufgrund des spezifischen Verlaufes eine Normierung auf $R$ (15000 Oe) verwendet. Von hohen, positiven Feldern kommend, steigt der Widerstand zunächst stark an (rote Kurve). Bei $H=1100$ Oe bricht der Widerstand ein und erreicht einen stabilen Wert. Für $H=-2500$ Oe steigt der Widerstand sprunghaft an, um dann wieder kontinuierlich mit dem Feld abzufallen. Dabei wird bei $H=-15 \mathrm{kOe}$ keine Sättigung erreicht. Wird das Feld wieder Richtung positiver Felder erniedrigt (grüne Kurve), ergibt sich ein symmetrisches Verhalten. Dabei ist die kontinuierliche Abnahme des Widerstandes für hohe Felder nichthysteretisch, der Unterschied zwischen der roten und der grünen Kurve im positiven Hochfeldbereich ist durch Temperaturschwankungen bedingt. Der starke Widerstandsrückgang findet bei $H=-1100$ Oe statt, der erneute Anstieg bei $H=2500$ Oe. Das Verhalten zeigt eine deutliche Hysterese. Im Kleinfeldbereich weist es 
Ähnlichkeiten mit dem des MgO-dotiertem FIB-Kontaktes auf (s. Abb. 7.14b). Allerdings kann in $\mathrm{CeO}_{2}$-dotierten Fall nur ein stabiles Minimum um $H=0$ Oe gefunden werden, im Gegensatz zu den zweien für den MgO-dotierten Fall.

Wird der Verlauf der nichthysteretischen, kontinuierlichen Widerstandsänderungen im Hochfeldbereich mit der Messung des Magnetowiderstandes der schmalen Struktur verglichen, so ergibt sich eine gute quantitative Übereinstimmung, was in Abbildung 7.17b) dargestellt ist. Im Kleinfeldbereich ergeben sich Unterschiede, da die FIB-Struktur einen negativen, die schmale Mikrostruktur einen positiven Kleinfeld-Magnetowiderstand aufweist.

\subsubsection{Zusammenfassung Magnetotransport}

In Tabelle 7.1 sind die wichtigsten Ergebnisse der Magnetotransport-Messungen, welche an den Bikristallkontakten vorgenommen wurden, zusammengefasst.

\begin{tabular}{|l|c|c|c|c|c|}
\hline Probe: & LSMO & LSMO & $\mathbf{1 0 \%} \mathbf{~ M g O}$ & $\mathbf{1 0 \%} \mathbf{~ M g O}$ & $\mathbf{2 0 \%} \mathbf{C e O}_{2}$ \\
\hline Bikristall Substrat: & $\mathrm{SrTiO}_{3}$ & $\mathrm{MgO}$ & $\mathrm{SrTiO}_{3}$ & $\mathrm{MgO}$ & $\mathrm{SrTiO}_{3}$ \\
\hline $\boldsymbol{c}$ [A] (LSMO): & 3,854 & 3,866 & 3,858 & 3,866 & 3,851 \\
\hline $\mathbf{T}_{C}$ [K]: & 350 & 305 & 310 & - & - \\
\hline$\rho_{\mathrm{KG}} / \rho_{\mathrm{epi}}$ @ 5 K, S1: & 2,5 & 1,2 & 2,4 & 1,0 & 1,3 \\
\hline$\rho_{\mathrm{S} 2} / \rho_{\mathrm{S} 1} @$ 5 K: & 250 & 3 & 20 & 40 & 6 \\
\hline max. LFMR, S1 [\%]: & 5,5 & 5 & 4 & 4 & $7-12$ \\
\hline max. LFMR, S2 [\%]: & 237 & 11 & 90 & 33 & 18 \\
\hline
\end{tabular}

Tabelle 7.1. Zusammenfassung der Magnetotransport-Ergebnisse, gewonnen an den Bikristallkontakten. S1(2): Struktur 1(2); epi: epitaktisch; KG: Korngrenze.

\subsection{Strominduzierte Effekte}

Durch die Verringerung der Strukturbreiten der Korngrenzen-Kontakte bietet sich die Möglichkeit, die magnetoresistiven Elemente mit hohen Stromdichten zu betreiben. Deshalb sollen die präparierten Kontakte in Hinblick auf strominduziertes Magnetisierungsschalten in lateral strukturierten Systemen untersucht werden. Bisher wurde das strominduzierte Magnetisierungsschalten nur mittels Punktkontakt-Messungen an unstrukturierten Proben oder an vertikal strukturierten Elementen untersucht.

\subsubsection{LSMO:MgO Korngrenzen-Kontakte}

Eine Untersuchung strominduzierter Prozesse wird am FIB-Kontakt der MgO-dotierten LSMO-Probe, deponiert auf einem MgO-Bikristall Substrat, vorgenommen. Dieses System zeigt einen LFMR bei tiefen Temperaturen, wie bereits in Abbildung 7.14 für $T=50 \mathrm{~K}$ gezeigt wurde. Abbildung 7.18 a) stellt den Widerstand dieses Kontaktes bei ebenfalls $T=50 \mathrm{~K}$ für verschiedene äußere Magnetfelder als Funktion der Stromstärke dar. Das 

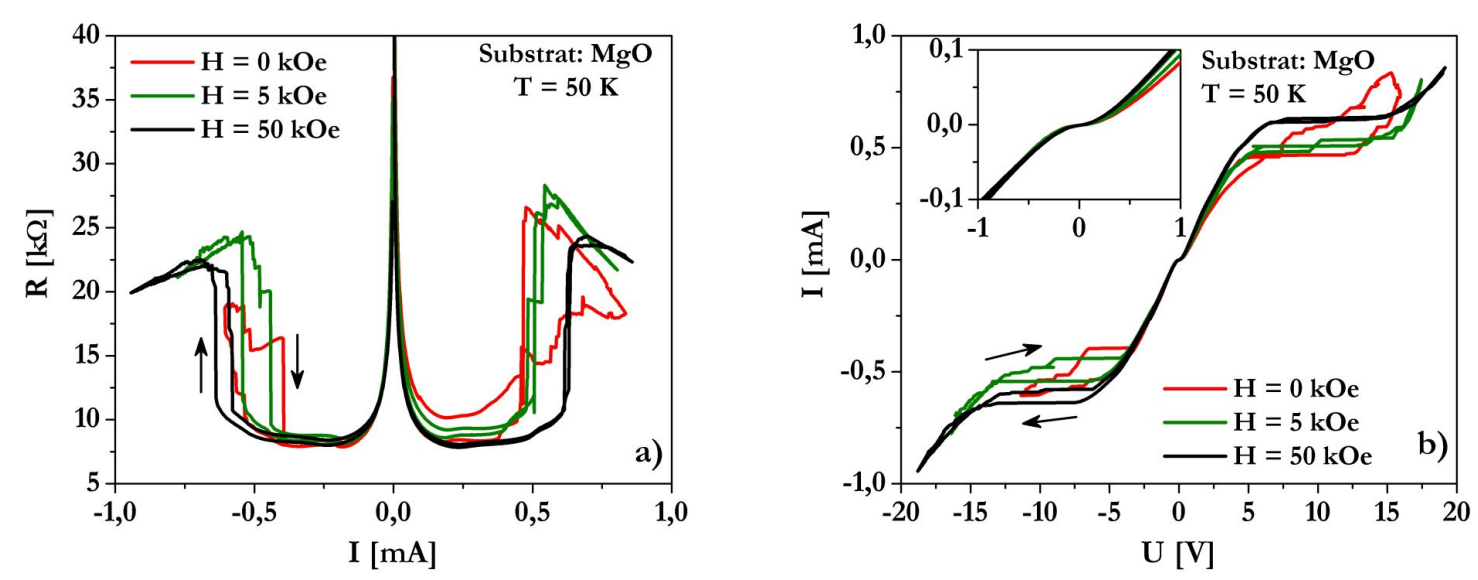

Abbildung 7.18. (LSMO $)_{0,9}:(\mathrm{MgO})_{0,1}$-Probe auf MgO-Bikristall Substrat. a) Widerstand als Funktion des Stromes bei $T=50 \mathrm{~K}$ in unterschiedlichen Magnetfeldern. b) StromSpannungs-Kennlinie der Daten nach a).

Experiment wird in Stromkontrolle betrieben, wobei die Stromstärke zunächst von kleinen positiven Werten auf den Maximalwert erhöht wird, anschließend kontinuierlich auf den negativen Maximalwert geändert und schließlich wieder auf kleine Stromstärken erniedrigt wird. Das Widerstandsverhalten in unterschiedlichen äußeren Magnetfeldern ist qualitativ vergleichbar, weshalb zunächst die Kurve für $H=50 \mathrm{kOe}$ vorgestellt wird: Für kleine Stromstärken ergibt sich ein vergleichsweise hoher Widerstand des Systems von ca. $40 \mathrm{k} \Omega$, welcher über den Magnetowiderstands-Effekt durch das äußere Magnetfeld beeinflussbar ist. Der Widerstand nimmt stark mit zunehmender Stromstärke ab und erreicht bei 0,20-0,25 mA ein Minimum von ca. $8 \mathrm{k} \Omega$. Mit weiter zunehmender Stromstärke steigt der Widerstand leicht auf einen Wert von ungefähr $10 \mathrm{k} \Omega$ bei $I=0,60 \mathrm{~mA}$. Bei $I=0,63 \mathrm{~mA}$ ist eine sprunghafte Erhöhung des Widerstandes auf $24 \mathrm{k} \Omega \mathrm{zu}$ beobachten, eine weitere Zunahme der Stromstärke auf $0,85 \mathrm{~mA}$ hat eine nur leichte Abnahme des Widerstandes auf $22 \mathrm{k} \Omega$ zur Folge. Die folgende Verringerung der Stromstärke führt zu einem fast identischen Verhalten des Widerstandes. Die sprunghafte Änderung tritt dabei zwar bei der gleichen Stromstärke auf, zeigt aber ein Stufe auf ungefähr der Hälfte der Widerstandsreduktion. Die hohe Widerstandsänderung bei $I \approx 0,63 \mathrm{~mA}$ ist für positive Werte somit nahezu hysteresefrei. Das geschilderte Verhalten zeigt sich auch für negative Stromstärken, allerdings weist die sprunghafte Widerstandsänderung für negative Stromstärken eine deutliche Hysterese auf. Bei $I=-0,63 \mathrm{~mA}$ findet der Widerstandsanstieg statt, wohingegen der Rückgang bei $I=-0,58 \mathrm{~mA}$ beobachtet wird.

Das geschilderte Verhalten ergibt sich qualitativ auch für $H=0 \mathrm{kOe}$, bzw. $H=5 \mathrm{kOe}$. Dabei zeigt sich, dass die zur abrupten Widerstandsänderung bei hohen Stromstärken nötige kritische Strömstärke $I_{C}$ systematisch mit dem äußeren Magnetfeld zunimmt. Im positiven Bereich können die Werte zu $I_{C}=0,47 \mathrm{~mA}(H=0 \mathrm{kOe}), I_{C}=0,50 \mathrm{~mA}(H=$ $5 \mathrm{kOe})$, bzw. $I_{C}=0,63 \mathrm{~mA}(H=50 \mathrm{kOe})$ ermittelt werden. Für negative Strömstärken ergibt sich beispielsweise für den Rückgang des Widerstands $I_{C}=-0,40 \mathrm{~mA}(H=0 \mathrm{kOe})$, $I_{C}=-0,44 \mathrm{~mA}(H=5 \mathrm{kOe})$, bzw. $I_{C}=-0,63 \mathrm{~mA}(H=50 \mathrm{kOe})$. Unter Verwendung einer kritischen Stromstärke von $I_{C}=0,50 \mathrm{~mA}$ lässt sich die kritische Stromdichte zu 
$J_{C} \approx 2,5 \cdot 10^{6} \mathrm{~A} / \mathrm{cm}^{2}$ berechnen.

Die Absolutwerte der Widerstände für hohe Stromstärken sind vergleichbar und zeigen keine systematische Abhängigkeit von äußeren Feld. Aufgrund der geringen verwendeten negativen Maximalstromstärke für $H=0 \mathrm{kOe}$ ist hier der maximale Widerstand möglicherweise nicht erreicht. Im Gegensatz zu den anderen Kurven weist diese Messung in Bezug auf die Widerstandswerte für hohe Ströme eine deutliche Asymmetrie auf. In diesem Zusammenhang fällt auf, dass die Widerstandsbestimmungen für hohe Magnetfelder rauschärmer sind und die unterschiedlichen Widerstandszustände besser definiert sind.

Die aus den $R(I)$-Messungen berechneten Strom-Spannungs-Kennlinien sind in Abbildung 7.18 b) aufgetragen. Die Widerstandsänderungen sind durch horizontale Spannungssprünge zu erkennen, da das Experiment in Stromkontrolle betrieben wird. Würde die Spannung als Ursache für die Widerstandsänderung aufgefasst, ergäbe sich auch hier eine systematische Abhängigkeit der kritischen Größe von äußeren Feld. Die kritischen Spannungen steigen mit dem äußeren Feld an.

Der Einsatz der Abbildung zeigt das Verhalten für kleine Spannungen, bzw. Stromstärken. Deutlich ist ein nichtlineares Verhalten zu erkennen, welches dem starken Abfall des Widerstandes für kleine Ströme in Abbildung 7.18 a) entspricht. Dabei ist wesentlich, dass die Verläufe für die jeweiligen Magnetfeldstärken geschlossen sind. Der Widerstand für kleine Stromstärken ändert sich somit auch nach einem zwischenzeitlichen Erreichen eines neuen Widerstandszustandes nicht.

Weitere strominduzierte Untersuchungen werden am $\mathrm{CeO}_{2}$-dotierten FIB-Kontakt vorgenommen. Hier verhindert jedoch die geringe Stabilität des Kontaktes eine detaillierte Charakterisierung, weshalb die erzielten Ergebnisse nicht aufgeführt werden. Dennoch widersprechen diese, soweit sie am stabilen Kontakt gewonnen werden konnten, nicht den Ergebnissen des MgO-dotierten FIB-Kontaktes. Vor Zerstörung des Kontaktes werden ebenfalls reversible Widerstandserhöhungen bei hohen Stromstärken beobachten. Dabei werden aber lediglich positive Stromstärken ohne Variation des äußeren Feldes und der Temperatur untersucht. Über weitere Abhängigkeiten kann entsprechend keine Aussage getroffen werden.

\subsection{Diskussion}

\subsubsection{Struktur}

Die Strukturanalysen der undotierten Proben ergeben die durch die Literatur [58, 59] zu erwartenden Ergebnisse eines epitaktischen Wachstums. In den Röntgendiffraktogrammen treten nur Reflexe des Typs (00l) auf, das Wachstum ist also c-Achsen-orientiert. Der cAchsenparameter ist für die auf STO deponierten Proben deutlich kleiner als der Wert für Volumenproben, die Probe steht unter mechanischen Spannungen. Anhand der Literatur 58,59 kann davon ausgegangen werden, dass diese Zugspannung für eine Schichtdicke von $50 \mathrm{~nm}$ über den gesamten Film besteht (s.a. Kap. 2.5.1). Für die auf $\mathrm{MgO}$ deponierten Schichten hingegen weicht der c-Achsenparameter kaum vom Volumenwert ab, die Probe ist weitgehend unverspannt, was ebenfalls erwartet wird [58,169. 
Die atomare Ordnung innerhalb der a-b-Ebene kann aus den REM- und TEMUntersuchungen abgeleitet werden. Oberflächenstrukturen, welche mittels des REM abgebildet werden, verlaufen entlang der kristallographischen Achsen der jeweiligen Halbkristalle des Substrats. Eine Abweichung von den durch das Substrat vorgegebenen Richtungen ist nicht zu beobachten. Die genaue Ursache dieser Oberflächenstrukturen bleibt dabei unklar. Die TEM-Analysen zeigen aber, dass sie nicht mit von der Oberfläche bis zum Substrat verlaufenden Defekten verbunden sind. Ein reines Inselwachstum kann daher nicht alleine ursächlich sein. Vielmehr deutet das alleinige Auftreten an der Oberfläche auf die Möglichkeit von Oberflächenrekonstruktionen oder auch auf den Abbau von Oberflächenspannungen, welche möglicherweise durch die Depositionsmethode bedingt sind. So ist durch die Präparationsbedingung ein flüssiger Film auf der Oberfläche vorstellbar, welcher die bereits solidifizierte Schicht aufgrund der spezifischen Oberflächenenergie nicht vollständig benetzt. Bei der Erstarrung könnten solche Hügelstrukturen erhalten bleiben. In jedem Fall treten diese Strukturen nur an der Oberfläche auf und haben so keinerlei Einfluss auf das Transportverhalten. Insgesamt kann das Wachstum auf den beiden Halbkristallen als epitaktisch bezeichnet werden.

Die Korngrenze, welche sich aus dem epitaktischen Wachstum auf den beiden Halbkristallen ergibt, ist sowohl in den REM- als auch in den TEM-Bildern deutlich zu erkennen. Die REM-Untersuchungen zeigen, dass die Korngrenze in unregelmäßigen Abständen auftretende Löcher aufweist. Die Ursache dieser strukturellen Inhomogenitäten ist wahrscheinlich die mangelhafte Qualität der Substrate. Abbildung 7.19a) zeigt einen größeren TEM-Ausschnitt der Korngrenze im Substrat: Es lassen sich Unregelmäßigkeiten der Struktur beobachten. Diese Strukturen zeigen keine regelmäßige atomare Ordnung auf und sind weitgehend amorph (Abb. 7.19b). Die Dimension dieser Fehlstellen entspricht dabei in etwa denen der in der Schicht beobachteten Löcher. Diese Löcher treten vermutlich in Bereichen auf, in denen solch unregelmäßige Strukturen im Substrat an dessen Oberfläche liegen.

Die Untersuchungen ergeben abgesehen von den Inhomogenitäten keinen genauen Aufschluss über die Mikrostruktur der Korngrenze. Aus den REM-Analysen kann geschlossen werden, dass die laterale Ausdehnung weniger als $10 \mathrm{~nm}$ beträgt, eine höhere Auflösung ist aber nicht zu erreichen. Im TEM verhindert die spezielle Geometrie der Lamelle eine detaillierte Aussage. Dennoch kann durch die ortsaufgelöste EDX-Messung eine wichtige Erkenntnis gewonnen werden: Selbst in den undotierten, auf MgO-Substraten deponierten Korngrenzen-Kontakten ist eine Anhäufung von $\mathrm{MgO}$ in der Korngrenze zu beobachten. Diese ist mit einem La-Defizit verbunden. Das MgO diffundiert vermutlich während der Deposition des LSMO aus dem Substrat in die Korngrenze.

Durch die Beobachtung der MgO-Agglomeration in der Korngrenze erscheint eine Anreicherung des $\mathrm{MgO}$ in der Korngrenze bei bewusster Dotierung wahrscheinlich. Diese Vermutung kann mittels der zur Verfügung stehenden Analysen weder verifiziert noch negiert werden, dennoch gibt es deutliche Hinweise auf eine berechtigte Annahme dieser These, die im Übrigen auch durch die Untersuchung an den Nanokompositen gestützt wird. Im Folgenden wird deshalb davon ausgegangen, dass bei Dotierung mit einem isolierenden Material, welches eine chemische Phasenseparation mit LSMO zeigt, sich dieses 

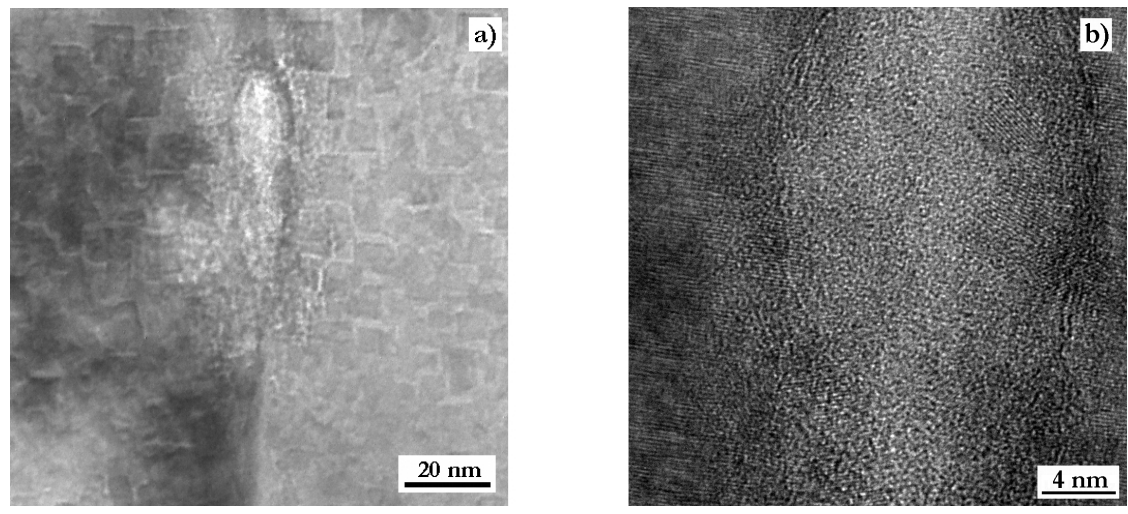

Abbildung 7.19. TEM-Querschnittsaufnahmen des MgO-Bikristall Substrats. a) Ungeordnete Struktur in der Korngrenze. b) Hochaufösende Aufnahme der Inhomogenität.

in der Korngrenze anreichert und im epitaktischen Teil Ausscheidungen gebildet werden. Diese Annahme beruht auch auf dem beträchtlichen Unterschied der Diffusionsrate zwischen dem epitaktischen Teil der Probe und der Korngrenze. So haben Blamire et al. 208 sowie Hammerl et al. 209] ebenfalls die Eigenschaften von definierten Korngrenzen in LCMO, bzw. $\mathrm{YBa}_{2} \mathrm{Cu}_{3} \mathrm{O}_{7-\delta}$ bewusst beeinflusst, indem sie einen Film anderer Zusammensetzung auf dem Korngrenzen-Kontakt deponiert haben und durch die erhöhte Korngrenzen-Diffusion diese nachträglich dotieren konnten. Im vorliegenden Fall wird die Korngrenze bewusst während der Deposition beeinflusst, was aufgrund der chemischen Phasenseparation möglich ist. Infolge der erhöhten Beweglichkeit gegenüber einer Diffusion im Volumenmaterial sollte diese Methodik effizienter ablaufen und zu einer definierteren Beeinflussung der Korngrenze führen, was insbesondere einen mögliche Gradienten in der Dotierung parallel zur Schichtnormalen bei der nachträglichen Dotierung betrifft. Dennoch kann über die genaue Mikrostruktur einer dotierten Korngrenze keine Aussage getroffen werden. Mögliche Szenarien, welche sich aus den Untersuchungen der Transporteigenschaften unter besonderer Berücksichtigung der systematischen Variation der Kontaktbreite ergeben, werden im weiteren Verlauf diskutiert (s.u.).

Die Annahme der Ausbildung von Ausscheidungen in epitaktischen Teilen der Probe wird durch Untersuchungen von Moshnyaga et al. [210] an epitaktischen LCMO:MgO Schichten unterstützt. Die Autoren zeigen scharfe Grenzflächen zwischen den beiden Phasen ohne Interdiffusion.

Über die mögliche Breite dieser MgO-Barriere in der Korngrenze kann mittels der EDX-Analyse wiederum aufgrund der Geometrie (gedrehte Lamelle) keine Aussage getroffen werden. Die aus den ortsaufgelösten Spektren ermittelte Erhöhung des MgO-Signals entspricht in der Breite abermals näherungsweise der Projektion auf die Abbildungsebene.

Die geringe Variation des LSMO $c$-Achsenparameters der auf STO deponierten Schichten bei Dotierung ist nicht auf eine Lösung von Mg- oder Ce-Atomen im Gitter zurückzuführen, da insbesondere im Fall des Mg keine Aufweitung des Gitters zu erwarten ist. Vielmehr zeigt sich, dass sich der LSMO-Gitterparameter dem der jeweiligen zweiten Phase annähert. So weist das $\mathrm{CeO}_{2}$ einen Gitterparameter von $a=5,41 \AA$ auf, welcher um 
einen Faktor 0,986 $\sqrt{2}$ größer ist als der Volumen-Gitterparameter des LSMO (s. Kap. 2.5.1. Im vorliegenden Fall ergibt sich eine Übereinstimmung von $0,993 \cdot \sqrt{2}$. Dabei weicht aber der für das $\mathrm{CeO}_{2}$ gemessene Gitterparameter im Gegensatz zu den Nanokompositen (s. Kap. 6.2.1) nicht von seinem Volumenwert ab. Offensichtlich findet keine La-Dotierung statt 193. Dieses könnte auf die komplexe Spannungsverteilung zwischen STO, LSMO und $\mathrm{CeO}_{2}$ zurückzuführen sein. Im Falle der MgO-Dotierung kommt es zu einer weiteren Aufweitung des LSMO in $c$-Richtung, was ebenfalls einer Annäherung an die zweite Phase entspricht. Über deren Spannungszustand kann keine Aussage getroffen werden, da wie für die LSMO:MgO-Nanokomposite keine MgO-Reflexe in den Röntgenspektren auftreten.

Für den Fall der auf MgO deponierten Schichten ist kein Einfluss der Dotierung auf den Gitterparameter zu beobachten. Dies ist einerseits ein erneuter Hinweis auf die chemische Phasenseparation, andererseits zeigt es, dass die oben diskutierten Gittervariationen möglicherweise mit einem durch das STO verursachten Spannungszustand verknüpft sind.

\subsubsection{Magnetotransport}

Die Geometrie von Struktur 1 erlaubt es, den Einfluss der Korngrenze auf das Transportverhalten direkt zu beobachten und zu extrahieren. Sowohl für die reinen LSMO-Proben als auch für die dotierten Systeme ergibt sich ein Unterschied. Wesentliche Merkmale der Korngrenze sind u.a. (s.a. Tabelle 7.1):

- Auftreten eines hysteretischen Kleinfeld-Magnetowiderstandes sowie eines nichthysteretischen Hochfeld-Magnetowiderstandes.

- Anstieg des spezifischen Widerstandes gegenüber einer Messung auf dem epitaktischen Teil der Probe.

- Schulter, bzw. Maximum im temperaturabhängigen Widerstandsverauf bei ca. 150$250 \mathrm{~K}$.

Dabei zeigen die Ergebnisse, dass der Einfluss der Korngrenze offensichtlich über eine Variation der Kontaktbreite gezielt gesteuert werden kann. Die folgende Diskussion wird zeigen, dass verschiedene magnetische Anisotropien (sowohl biaxiale als uniaxial) eine wichtige Rolle für die Form und den Wert des beobachteten LFMR spielen können. Andererseits könnten die bereits oben diskutierten Inhomogenitäten der Korngrenzen für das beobachtete unterschiedliche Verhalten verantwortlich sein.

Zunächst wird das Verhalten der undotierten Proben im Detail diskutiert. Die Magnetisierung der auf STO deponierten Probe (s. Abb. 7.5 zeigt unterhalb $T_{C}=350 \mathrm{~K}$ das erwartete ferromagnetische Verhalten. Die Phasenübergangstemperatur ist dabei aufgrund der mechanischen Spannungen gegenüber dem Volumenwert verringert. Dennoch weist die Magnetisierungskurve einen vergleichsweise scharfen Übergang auf, es existieren keine Hinweise auf eine mögliche Verteilung von unterschiedlichen Phasenübergangstemperaturen. Für den epitaktischen Teil ergibt sich auch oberhalb von $T_{C}$ ein Anstieg des Widerstandes, wie er aus dem Phasendiagramm (a. Abb. 2.7) erwartet wird. Auch im übrigen Temperaturbereich zeigt der Widerstand das erwartete Verhalten [12]. Neben der Erhöhung des Widerstandes hat die Berücksichtigung der Korngrenze auch ein aktiviertes 
Widerstandsverhalten für $T \geq T_{C}$ zur Folge, welches auf einen isolierenden Bereich in der Korngrenze hindeutet. Die Ursache der Schulter im temperaturabhängigen Widerstandsverlauf wird in der Literatur kontrovers diskutiert. Ziese [31] sieht die Schulter als Hinweis auf eine hauptsächlich antiferromagnetisch geordnete Spinstruktur an der Grenzfläche.

\section{Magnetotransport des breiten Kontaktes}

Der Magnetowiderstand der breiten Struktur unter Berücksichtigung der Korngrenze ist vergleichsweise gering. Dies gilt für alle präparierten Kontakte, unabhängig von Dotierung und Substrat. Bei tiefen Temperaturen wird ein maximaler LFMR von $7 \%$ beobachtet $\left(\mathrm{CeO}_{2}\right.$-dotierter Kontakt), wobei in keiner der Magnetowiderstandskurven ein Schalten zwischen definierten Widerstandszuständen beobachtet wird. Vielmehr können die Kurven aufgrund von Vergleichen mit simulierten Verläufen von Gunnarsson et al. 211,212 durch eine Rotation der Magnetisierungen der beiden Halbkristalle erklärt werden. Die Berechnungen der Autoren gehen zwar von einer zu den hier vorliegenden Messungen abweichenden Geometrie aus (Magnetfeld senkrecht zur Korngrenze), dennoch ergibt sich eine hohe qualitative Übereinstimmung in der Form der Widerstandskurven. Dabei sind aber zusätzliche Unterschiede in den experimentellen Ergebnissen zu berücksichtigen. Abbildung 7.20 zeigt das unterschiedliche Hystereseverhalten der breiten Strukturen für eine LSMO-Probe auf einem MgO-Substrat im Vergleich mit einer LSMO:MgO-Probe auf STO. In beiden Fällen ist ein kontinuierliche Änderung des Widerstandes zu beobachten: für die Probe auf MgO vor Erreichen des Maximums, für die Probe auf STO nach Überschreiten des Maximums. Dieses grundsätzlich verschiedene Verhalten kann durch einen Unterschied in der Anisotropie erklärt werden. Aufgrund der unverspannten Struktur des LSMO bei Deposition auf MgO ist hier innerhalb der Schichtebene keine Anisotropie vorhanden. Die Existenz einer biaxialen Anisotropie bei auf STO deponierten Schichten ist jedoch bekannt 213,214,215. Dabei verläuft die leichte Achse der Magnetisierung entlang der [110]-Richtung. Nach Gunnaarsson et al. 211, 212] ist eine Rotation der Magnetisierungen nach Überschreiten des Maximums im MR nur durch die biaxiale Anisotropie im verspannten LSMO zu erklären. Ohne diese biaxiale Anisotroie zeigt sich ein Verlauf, der dem des unverspannten LSMO qualitativ ähnlich ist. Trotz der unterschiedlichen Geometrien der experimentellen Ergebnisse und der von Gunnarsson und Mitarbeitern durchgeführten Simulationen kann das unterschiedliche Verhalten vermutlich auf die verschiedenen Anisotropien im LSMO zurückgeführt werden.

Die Größenordnung des maximalen LFMR der breiten Struktur bei tiefen Temperaturen von 4-7\% ist vergleichbar mit Angaben in der Literatur, in denen ähnliche Formen der Hysterese beobachtet werden [134,135]. Die dort beschriebenen Ergebnisse werden an Kontakten mit einer Breite von 5-6 $\mathrm{m}$ erzielt, wohingegen die hier verwendeten Kontakte $50 \mu \mathrm{m}$ breit sind. Es ergeben sich in dieser Arbeit keine Hinweise auf eine nichtlineare Strom-Spannungs-Kennlinie, was aber nur für kleine Spannungen analysiert wurde. In den oben zitierten Veröffentlichungen wird keine Aussage über die Charakteristik der Kennlinien getroffen. Aus den Rauschdaten von Mathieu et al. [134 kann aber geschlossen werden, dass diese ebenfalls linear sind. Auch die Flächen-Widerstandsprodukte sind von 


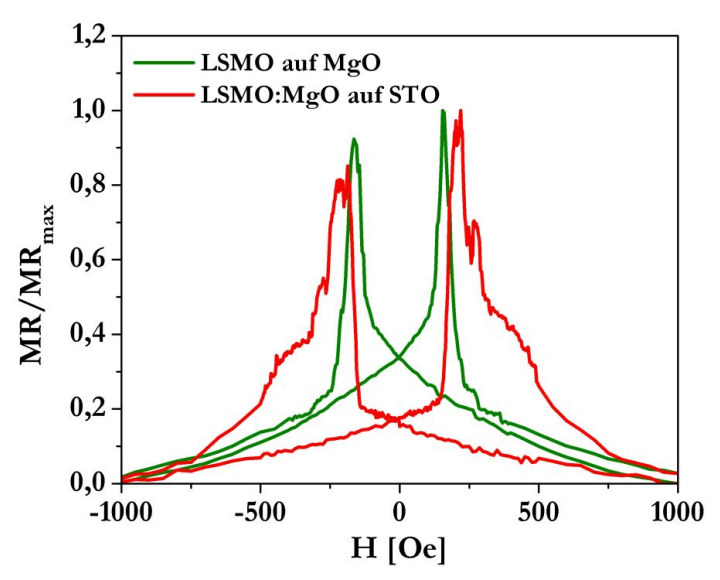

Abbildung 7.20. Normiertes Magnetowiderstandsverhalten eines auf $\mathrm{MgO}$ deponierten LSMO-Kontaktes im Vergleich mit einem auf STO deponierten LSMO:MgO-Kontakt.

vergleichbarer Größenordnung: Gunnarsson et al. [135] berichten von Werten der Größenordnung $R \cdot A_{K G} \approx 3 \mu \Omega \mathrm{cm}^{2}$. Für die breiten Strukturen ergeben sich in dieser Arbeit Werte der Größenordnung $R \cdot A_{K G} \approx 1,3 \mu \Omega \mathrm{cm}^{2}$.

\section{Einfluss der Kontaktbreite}

Die Verringerung der Strukturbreite hat einen immensen Einfluss auf das Transportverhalten der Proben, was im Folgenden an der auf STO deponierten LSMO-Probe genauer diskutiert wird (s. Abb. 7.6 7.8). Die in der breiten Struktur beobachtete Schulter dominiert im temperaturabhängigen Widerstandsverlauf und wird zum Maximum, dennoch ist der Einfluss des epitaktischen Teils der Probe durch eine geringe Änderung der Steigung im Widerstand bei $T_{C}$ weiterhin zu erkennen. Neben einer deutlichen Steigerung des spezifischen Widerstandes ändern sich auch Form und Wert des Magnetowiderstandes. Im magnetfeldabhängigen Widerstand zeigen sich stabile Plateaus, welche auf eine geänderte magnetische Anisotropie hindeuten. Ein scharfes Schalten zwischen definierten Niveaus wird bei einer uniaxialen Anisotropie mit dem Magnetfeld in leichter Richtung erwartet. Philipp et al. [216 haben ebenfalls einen LFMR vergleichbarer Form und Größe an LCMO Korngrenzen-Kontakten beobachtet und zeigen, dass die Form auf eine uniaxiale Anisotropie mit der Korngrenze als leichter Achse zurückgeführt werden. Eine detaillierte mikroskopische Begründung für das Auftreten der leichten Achse entlang der Korngrenze und der damit verbundenen uniaxialen Anisotropie wird aber nicht gegeben. Offensichtlich kommt es für die hier untersuchten Korngrenzen-Kontakte durch die Verringerung der Strukturbreite zu einer Änderung der magnetischen Anisotropie. Die Tatsache, dass die uniaxiale Anisotropie von Philipp et al. bereits bei Strukturgrößen von $30 \mu \mathrm{m}$ beobachtet wird, was eher der Geometrie der breiten als jener der schmalen Struktur entspricht, lässt die Frage nach der Ursache für den Übergang der Anisotropien aufkommen. Denkbar ist, dass die biaxiale Anisotropie im LSMO, welche im LCMO gegebenenfalls geringer Größenordnung ist, für große Strukturbreiten gegenüber der uniaxialen Anisotropie dominiert und so der LFMR durch Rotation der Magnetisierungen bestimmt wird. Gegen diese The- 
se spricht, dass die oben zitierten Ergebnisse von Mathieu et al. [134] und Gunnarsson et al. [135, welche einen geringen LFMR auf Basis der biaxialen Anisotropie zeigen, an schmalen Strukturen gemessen werden. Vielmehr scheint eine Änderung des Charakters der Korngrenze, vermutlich aufgrund räumlicher Inhomogenitäten, wie sie in den Strukturuntersuchungen beobachtet werden, für das geänderte Verhalten verantwortlich. Für die Richtigkeit dieser Hypothese gibt es mehrere Hinweise: Das deutlichste Indiz ist der starke Anstieg des spezifischen Widerstandes, welcher ohne eine Variation der Korngrenzeneigenschaften nicht erklärt werden kann. Weiterhin zeigen die Strom-Spannungs-Kennlinien im Gegensatz zu den breiten Strukturen ein stark nichtlineares Verhalten, welches gut durch das auf einem Tunnelprozess basierenden Glazman-Matveev-Modell beschrieben werden kann. Das geänderte Magnetowiderstandsverhalten ergibt sich jedoch nicht zwangsläufig bei Verringerung der Strukturbreite, wie die Ergebnisse für die auf $\mathrm{MgO}$ deponierte LSMO-Probe zeigt. Die Verminderung der Kontaktgröße bedingt für diesen Fall nur einen geringfügigen Anstieg des Widerstandes, die Form des LFMR ändert sich ebenfalls nur leicht und weist im Gegensatz zum scharfen Schalten zwischen zwei definierten Widerstandswerten deutliche Kennzeichen einer Rotation der magnetischen Momente auf. Der LFMR vergrößert sich nur leicht auf $11 \%$ und liegt damit deutlich unterhalb des Wertes für die schmale Struktur der auf STO deponierten Probe (237\%). Sowohl für die breite als auch für die schmale Struktur ist keine Stromabhängigkeit des LFMR zu beobachten, die Schulter im temperaturabhängigen Widerstandsverlauf geht überdies durch die Verringerung der Strukturbreite zurück.

Eine systematische Betrachtung der durch Verringerung der Kontaktbreite erzielten Ergebnisse aller untersuchten Proben ergibt, dass die Erhöhung des spezifischen Widerstandes ein geeignetes Maß für die Beurteilung der Transporteigenschaften darstellt. Erhöht sich der spezifische Widerstand deutlich $\left(\rho_{\mathrm{S} 2} / \rho_{\mathrm{S} 1} \gg 1\right)$, ist ein starker Anstieg der Schulter im temperaturabhängigen Widerstandsverlauf zu beobachten, s.d. diese auch den Verlauf im Form eines Maximums dominieren kann [s. z.B. Abb. 7.6b) und 7.11b)]. Weiterhin ergibt sich ein nichtlinearer Zusammenhang von Strom und Spannung [s. beispielsweise Abb. 7.7 b) und 7.12 a)], welcher gut durch das Glazman-Matveev-Modell beschrieben werden kann. Der LFMR, welcher eine vergleichsweise hohe Strom- bzw. Spannungsabhängigkeit aufweist, zeigt ein stufenförmiges Verhalten, welches auf eine uniaxiale Anisotropie mit der Korngrenze als leichte Magnetisierungsachse zurückgeführt werden kann [s. Abb. 7.6 b), 7.11 b) und 7.13 b]. Die erzielten Widerstandsänderung sind deutlich erhöht und sind maximal bei größter Änderung des spezifischen Widerstandes $(230 \%$ bei $\rho_{\mathrm{S} 2} / \rho_{\mathrm{S} 1}=250$, s. Tab. 7.1).

Ändert sich der spezifische Widerstand bei Verminderung der Kontaktbreite nur wenig, so ist keine wesentliche Änderung in den Transporteigenschaften zu beobachten. Diese Tatsache bestätigt sich auch durch weitere Experimente an Bikristallkontakten, welche nicht in den Ergebnissen aufgeführt sind.

Eine mögliche Erklärung der Variation der Transporteigenschaften bei Beeinflussung der Kontaktbreite ergibt sich aus strukturellen Inhomogenitäten der Korngrenze. Diese werden auf einer Längenskala im Mikrometer-Bereich beobachtet. Die Kontaktbreite der Struktur 2 liegt in eben diesem Größenbereich und ist dementsprechend sensitiv auf die 
genaue Verteilung der Inhomogenitäten. Ein breiterer Stromstreifen hingegen ist unempfindlich hinsichtlich deren genauer Anordnung, es ergeben sich reproduzierbare Ergebnisse.

Die genaue Beschaffenheit dieser Inhomogenitäten ist allerdings unklar. Ein Einfluss der beobachteten Löcher ist zwar denkbar, kann aber nicht direkt nachgewiesen werden. So haben beispielsweise REM-Untersuchungen des schmalen LSMO-Kontaktes auf STO keine Variation entlang der Korngrenze gezeigt, wohingegen für andere Kontakte, welche ebenfalls einen hohen spezifischen Widerstand aufweisen, Inhomogenitäten beobachtet werden. Detaillierte TEM-Analysen der Korngrenze in Aufsicht könnten zur weiteren Aufklärung beitragen.

Dem durch Variation der Kontaktbreite geänderten Verhalten des Magnetotransports steht für den breiten $\mathrm{CeO}_{2}$-dotierten Korngrenzen-Kontakt das mittels Temperatur geänderte Verhalten gegenüber (s. Abb. 7.16). Für tiefe Temperaturen wird das typische, auf eine Rotation der jeweiligen Magnetisierungen zurückzuführende Verhalten für breite Strukturen beobachtet. Für höhere Temperaturen hingegen zeigt sich ein Zusammenhang mit dem Ummagnetisierungsprozess, welcher typischerweise bei einem schmalen Kontakt auftritt. So sind beispielsweise die Felder maximalen Widerstandes bei $T=50 \mathrm{~K}$ für die breite und schmale Struktur annähernd identisch und auch die Form stimmt qualitativ überein (s. Abb. 7.16b). Ein ähnliches Verhalten ist ebenfalls für den schmalen Kontakt bei $T=5 \mathrm{~K}$ zu beobachten. Zusätzlich zu den typischen, scharf definierten Widerstandsplateaus treten weitere Widerstandsmaxima bei den Feldern auf, an den der Widerstand der breiten Struktur maximal ist. Offensichtlich kommt es zu einer Überlagerung der beiden Verhaltensweisen sowohl im breiten als auch im schmalen Kontakt.

Insbesondere das temperaturabhängige Auftreten der beiden unterschiedlichen Verhaltensweisen der breiten Struktur kann nicht durch eine strukturelle Änderung der Korngrenze erklärt werden. Möglicherweise hat der durch das $\mathrm{CeO}_{2}$ geänderte Spannungszustand (s. Kap. 7.4.1) einen Einfluss auf das Anisotropieverhalten. Da die biaxiale Anisotropie im LSMO durch die mechanischen Spannungen aufgrund des epitaktischen Wachstums auf STO verursacht wird, ist ein weiterer Einfluss durch die mechanischen Spannungen aufgrund der $\mathrm{CeO}_{2}$-Dotierung wahrscheinlich. Gegebenenfalls führt dies zur Verstärkung der uniaxialen Anisotropie mit der Korngrenze als leichte Magnetisierungsachse, bzw. zur Abschwächung der biaxialen Anisotropie. Eine unterschiedliche Temperaturabhängigkeit der Anisotropiekonstanten könnte schließlich zu einer Änderung des Verhaltens bei Temperaturvariation führen. Möglicherweise weist dieser Kontakt auch Inhomogenitäten der Barriere auf einer größeren Längenskala als die anderen untersuchten Kontakte auf, was ebenfalls zu abweichendem Verhalten führen würde.

\section{Magnetotransport des schmalen Kontaktes}

Im Folgenden wird der Zustand mit geänderten Transportverhalten weiter charakterisiert. Die Asymmetrie der Magnetowiderstandskurven, welche besonders für die LSMO-Probe auf STO, aber auch für andere Proben beobachtet wird, ist vermutlich auf leichte Unterschiede in der Mikrostruktur nahe der Korngrenze in den beiden Halbkristallen zurückzuführen. In einem perfekt symmetrischen Kontakt mit der Korngrenze als leichter 
Magnetisierungsachse, welche genau parallel zum äußeren Feld verläuft, sollte kein LFMR erkennbar sein, da sich die Magnetisierungen beider Halbkristalle gleich verhalten und sich die relative Orientierung der Magnetisierung entsprechend nicht ändert. Pinningzentren nahe der Korngrenze können das Magnetisierungsverhalten verkomplizieren und als nicht reproduzierbar erscheinen lassen [131. Entsprechend können auch verschiedene Konfigurationen stabil sein, welche einen unterschiedlichen Widerstand zur Folge haben. Aus dieser Argumentation folgen jedoch lediglich unterschiedliche Widerstandszustände, eine Asymmetrie in der Breite der jeweiligen Zustände kann aber nicht erklärt werden. Diese folgt nur aus einer relativen Verschiebung der jeweiligen Widerstandshysteresen gegeneinander. Möglicherweise ist die Grenzflächenmagnetisierung an eine antiferromagnetisch geordnete Korngrenze gekoppelt, s.d. es durch eine Kühlung im magnetischen Feld zu einer zusätzlichen Anisotropie durch den Antiferromagneten kommt. Dieser Effekt wird auch als exchange bias bezeichnet 217,218. In diesem Sinne kann die unterschiedliche Breite der Widerstandshysterese als ein Hinweis auf eine antiferromagnetische Ordnung in der Korngrenze angesehen werden, wie sie bereits von Ziese [31] im Zusammenhang mit der Schulter im temperaturabhängigen Widerstandsverlauf vermutet wird.

Wie bereits dargestellt, kann der Verlauf der Strom-Spannungs-Kennlinien gut durch das Glazman-Matveev-Modell [121] wiedergegeben werden. Hieraus kann auch direkt die vergleichsweise hohe Abhängigkeit des LFMR von der Spannung abgeleitet werden. Das Glazman-Matveev-Modell beschreibt das Tunneln über Zwischenzustände in der Barriere. Mit zunehmender Spannung tragen die inelastischen Kanäle mehr zum Tunnelstrom bei, da immer mehr Zwischenzustände energetisch zugänglich werden. In den inelastischen Kanälen ist die Spin-Streuung deutlich erhöht, wodurch sich der Magnetowiderstand insgesamt verringert 219], was auch experimentell bestätigt wird.

Ähnlich wie für die Spannung könnte auch für das Temperaturverhalten argumentiert werden 219. Entsprechend sollte der LFMR mit zunehmender Temperatur abnehmen, zumal zusätzlich die Spinpolaristation der Elektroden abnimmt. Dies wird aber nur in Ausnahmefällen beobachtet, vielmehr stellt das Auftreten eines Maximums im temperaturabhängigen Verlauf des LFMR die Regel dar. Unter der Annahme einer bei tiefen Temperaturen maximalen Spinpolarisation sowie temperaturunabhängiger Eigenschaften der Barriere kann der mit zunehmender Temperatur ansteigende LFMR nur über einen zunehmenden Winkel zwischen den Magnetisierungen der beiden Halbkristalle erklärt werden. Ursächlich könnten Hystereseeffekte sein. Diese Erklärungsmöglichkeit erscheint jedoch unwahrscheinlich, da das Maximum im LFMR auch bei Messungen an den breiten Strukturen auftritt. Die jeweiligen Ummagnetisierungsvorgänge sind aber grundsätzlich verschieden, woraus geschlossen werden kann, dass der Effekt nicht durch einen speziellen Ummagnetisierungvorgang bedingt ist, da er sowohl für die biaxiale als auch für die uniaxiale Anisotropie auftritt. Park et al. 115 haben gezeigt, dass die OberflächenSpinpolarisation von LSMO stetig mit zunehmender Temperatur abnimmt. Entsprechend kann das Maximum im LFMR nur über eine Temperaturabhängigkeit der Korngrenzeneigenschaften erklärt werde.

Die genaue magnetische Ordnung in der Korngrenze wird in der Literatur unterschiedlich bewertet. Einerseits existieren Modelle, nach denen die Korngrenze keinen isolie- 
renden Charakter hat [137|, welche durch magnetische Untersuchungen der Korngrenze gestützt werden, in denen eine Erhöhung der Curie-Temperatur der Korngrenze beobachtet wird [133]. Andere Modelle hingegen basieren hauptsächlich auf Modifikationen eines spin-polarisierten Tunnelprozesses, also auf einer isolierenden Barriere [27, 136, 28, 31. Ursprünglich wurde dabei ein paramagnetischer Zustand der Barriere mit hoher Defektdichte angenommen 28. Systematische Untersuchungen auch unter Anwendung von $1 / f$ Rauschanalysen 136 haben aber gezeigt, dass eine magnetische Ordnung der lokalisierten Momente, vermutlich in Form eines Spinglases, in der Barriere existiert.

Diese spezielle Form der magnetischen Ordnung ist möglicherweise für den ungewöhnlichen Verlauf des temperaturabhängigen LFMR verantwortlich. Wird ein Spinglas ohne äußeres Magnetfeld gekühlt (engl.: zero field cooled, ZFC) und anschließend in moderaten Felder die Magnetisierung für zunehmende Temperaturen ermittelt, so ergibt sich ein Maximum in der Magnetisierung [220]. Die Messung des LFMR findet ebenfalls nach Kühlung ohne äußeres Feld statt und kann demnach als ZFC-Messung betrachtet werden. Innerhalb der Barriere ist die Magnetisierung nach diesem Bild bei einer bestimmten Temperatur maximal. Ausgehend von einem mehrstufigen Tunnelprozess über Defektzustände in der Barriere, deren magnetische Momente das Spinglas bilden [136], ist die Spinstreuung mit der Magnetisierung der Korngrenze verbunden. Bei maximaler Magnetisierung ist die Spinstreuung einer Spinsorte minimiert, was unter Umständen zu einer Erhöhung des LFMR führen könnte. Bei tieferen Temperaturen hingegen erhöht sich die Spinstreuung dieser Spinsorte, der LFMR ist geringer. Für höhere Temperaturen kommen weitere Effekte wie der Abfall der Spinpolarisation zum Tragen - der LFMR nimmt ab.

Ein direkter Nachweis für diese These kann nicht gegeben werden. Eine einfache Verifikation läge in der Bestimmung des LFMR nach Kühlung in einem hohen magnetischen Feld (engl.: field cooled, FC). In diesem Fall zeigt sich kein Maximum in der Magnetisierung eines Spinglases, sie ist weitgehend konstant [220]. Verschwindet das Maximum im temperaturabhängigen LFMR, so kann es als Hinweis auf eine magnetische Ordnung in Form eines Spinglases angesehen werden. Da alle Messungen in dieser Arbeit aber vom Typ ZFC sind, kann hier kein Nachweis geliefert werden.

\section{Dotierung der Korngrenze}

Die Dotierung der Proben mit einem isolierenden Material zieht für die breiten Strukturen eine Erhöhung des Widerstandes nach sich, was sowohl für den epitaktischen Teil der Probe als auch für die Korngrenze gilt. Da sich das qualitative Verhalten auf dem epitaktischen Teil der Probe nicht ändert, kann geschlossen werden, dass eine chemische Phasenseparation zwischen LSMO und der isolierenden Phase auftritt und Ausscheidungen des Isolators in Form von statischen Defekten den Widerstand erhöhen. Eine Agglomeration der isolierenden Phase in der Korngrenze, wie sie oben bereits diskutiert wurde, kann für die Widerstandserhöhung der Korngrenze ursächlich sein. Andererseits ist die Widerstandserhöhung der Korngrenze eher gering und skaliert in ähnlicher Weise wie der Widerstand des epitaktischen Teils. Würde das isolierende Material die beiden LSMO-Halbkristalle vollständig trennen, wäre ein deutlicherer Anstieg des Widerstandes 

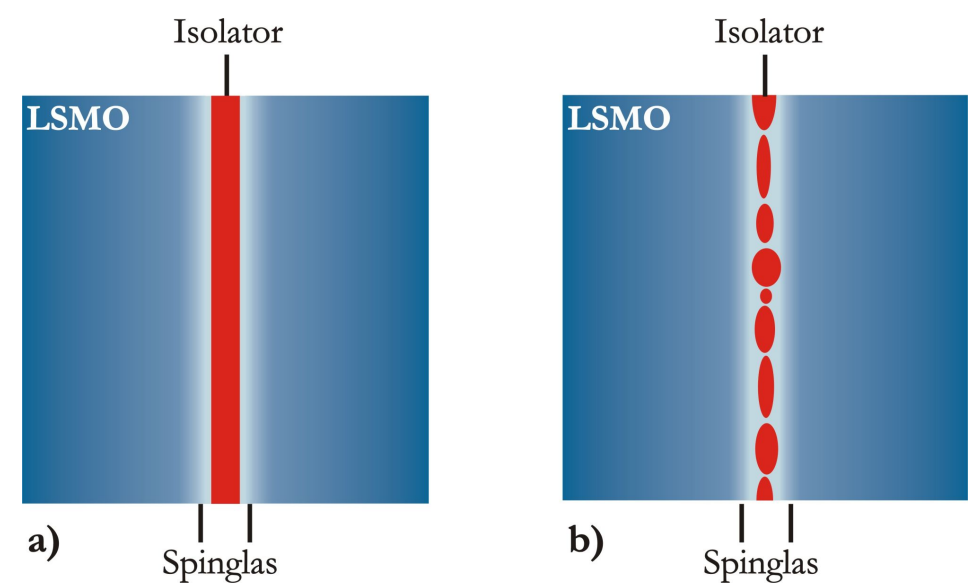

Abbildung 7.21. Mögliche Szenarien der Agglomeration der isolierenden Phase in der Korngrenze (schematische Aufsicht, innerhalb der beiden Halbkristalle wird die isolierende Phase vernachlässigt). a) Der Isolator ist durchgängig und trennt die beiden Halbkristalle. b) Die isolierende Phase ist nicht durchgängig und trennt die Halbkristalle nicht vollständig.

der Korngrenze aufgrund der exponentiellen Transmissionswahrscheinlichkeit der Elektronen beim Tunnelprozess zu erwarten. Weiterhin hat die Dotierung der Korngrenze keinen Einfluss auf den qualitativen Verlauf des temperaturabhängigen Widerstandes sowie auf den LFMR. So ist beispielsweise für den MgO-dotierten LSMO-Kontakt auf STO (s. Abb. 7.107 .12 eine Schulter im temperaturabhängigen Widerstandsverlauf bei ca. $200 \mathrm{~K}$ zu beobachten, welche im epitaktischen Teil nicht auftritt. Die Widerstandshysterese ist zwar deutlich härter, was möglicherweise durch Pinning-Effekte der MgO-Ausscheidungen erklärt werden kann [196], andererseits ist der Absolutwert des LFMR fast identisch und zeigt ebenfalls ein Maximum im temperaturabhängigen Verlauf. Auch die Verringerung der Strukturbreite zeigt einen zur undotierten Probe identischen Effekt: Der Widerstand steigt stark an, ebenso der LFMR, welcher von scharf definierten Plateaus bestimmt wird. In beiden Fällen wird die Strom-Spannungs-Kennlinie gut durch das Glazman-MatveevModell beschrieben.

Es ergeben sich zwei mögliche Szenarien der Dotierung der Korngrenze (s. Abb. 7.21), welche beide in einem gewissen Rahmen das beobachtete Verhalten erklären können. Zunächst sei der angestrebte Fall einer homogenen, durchgehenden Barriere betrachtet [Abbildung 7.21 a)]. In diesem Fall ist der Transportmechanismus über die Korngrenze unstrittig: er wäre dominiert vom spinabhängigen Tunneln der Leitungselektronen über die isolierende Barriere. Dennoch könnte ein möglicherweise gestörter Bereich an der Korngrenze (z.B. in Form des oben diskutierten Falls des Spinglases) den Transport beeinflussen und beispielsweise das Maximum im LFMR verursachen. Wenn dieses Szenario zutrifft, so hätte dies Konsequenzen für die allgemeinen Eigenschaften der Korngrenze. Da sich durch das Einfügen einer isolierenden Barriere die wesentlichen Merkmale nur marginal ändern, muss auch ohne eine zweite Phase ein isolierender Bereich vorhanden sein. In diesem Fall wäre das Modell nach Evetts et al. [137| widerlegt (s. Kap. 3.4) und ein auf einem Tunnelprozess basierender Transportmechanismus für einen undotierten Bikristall-Kontakt indirekt nachgewiesen. 
Neben diesem Szenario ist als weitere Möglichkeit denkbar, dass die isolierende Phase nicht zusammenhängend in der Korngrenze agglomeriert und somit die beiden LSMOHalbkristalle nicht vollständig separiert [s. Abb. $7.21 \mathrm{~b}$ )]. In diesem Fall würde der Transport fast ausschließlich über die direkten Kontakte der LSMO-Halbkristalle (engl.: pinholes) stattfinden, welche im Gegensatz zum Transport über die MgO-Barriere einen vergleichsweise geringen Widerstand aufweisen. Dabei ist der mittlere Abstand der pinholes deutlich kleiner als die verwendeten Kontaktbreiten, s.d. die Variation der Strukturgröße nur auf Längenskalen der durch das Substrat verursachten strukturellen Inhomogenitäten stattfindet, nicht aber auf der Längenskala des mittleren Abstandes der pinholes. Entsprechend ändert sich der Magnetotransport über die Korngrenze nur unwesentlich zum undotierten Fall, da er auch im dotierten Fall größtenteils über einen undotierten Bereich stattfindet.

Direkte Hinweise für eines der beiden entwickelten Szenarien existieren nicht. Dennoch erscheint das zweite Modell aufgrund der nur geringen Widerstandserhöhung wahrscheinlicher. Gegebenenfalls ist ein Anteil der isolierenden Phase von bis 20\% (im Falle von $\mathrm{CeO}_{2}$ ) zu gering, um die Korngrenze vollständig zu füllen. Systematische AufsichtsTEM-Analysen, welche eine große experimentelle Herausforderung darstellen, könnten zur Klärung der Korngrenzenstruktur beitragen.

\section{FIB-Kontakte}

Das Magnetowiderstandsverhalten der beiden präparierten FIB-Strukturen (s. Abb. 7.14 und 7.17) zeigt ein einheitliches Bild. Neben dem typischen, nichthysteretischen Widerstandsverlauf für hohe Felder, welcher auch für Struktur 2 beobachtet wird und entsprechend kein besonderes Merkmal des FIB-Kontaktes darstellt (s. Abb. 7.17b), ergibt sich in beiden Fällen ein Widerstandsminimum bei kleinen Feldern. Der ungeänderte Hochfeldmagnetowiderstand, welcher üblicherweise sowohl durch ein CMR-Verhalten (s. Kap. 2.2 als auch durch das Ausrichten der lokalisierten magnetischen Momente in der Barriere [136 erklärt wird, lässt darauf schließen, dass der Kontakt nicht durch die FIBPräparation in Mitleidenschaft gezogen wird. Auch temperaturabhängige Widerstandsmessungen zeigen das erwartete Verhalten. Eine Bewertung der Phasenübergangstemperatur kann dabei jedoch nicht vorgenommen werden, da die Kontakte nur unterhalb von Raumtemperatur charakterisiert wurden. Gunnarsson et al. 221 berichten von einer Verschiebung der Metall-Isolator-Übergangstemperatur um ca. $20 \mathrm{~K}$, welche sie auf die Implantation von Ga-Ionen durch die FIB-Behandlung zurückführen.

Offensichtlich kommt es durch die weitere Verjüngung des Korngrenzenkontaktes zu einem geänderten Kleinfeldverhalten. Es ergibt sich ein negativer Magnetowiderstand. Entsprechend muss geschlossen werden, dass der positive LFMR, verursacht durch das spinpolarisierte Tunneln der Leitungselektronen über die Barriere, entweder verschwindet oder durch einen weiteren, größeren Effekt überdeckt wird. Auffallend ist dabei, dass die Widerstandsverringerung schon vor Überschreiten des Nullfeldes stattfinden, also keine Hysterese im engeren Sinne beobachtet wird. Dennoch zeigen die kritischen Felder eine Abhängigkeit von der Breite des Kontaktes: Für den breiteren Kontakt (LSMO:MgO auf 
$\mathrm{MgO}, d=400 \mathrm{~nm}, \mathrm{Abb} .7 .14$ weist das Minimum zwischen den abrupten Widerstandsänderungen eine Breite von $\Delta H=370$ Oe auf, für den schmaleren Kontakt (LSMO:CeO ${ }_{2}$, $d=250 \mathrm{~nm}, \mathrm{Abb} .7 .17$ ) hingegen beträgt der Abstand zwischen den inneren Widerstandsänderungen $\Delta H \approx 2200$ Oe, zwischen den äußeren sogar $\Delta H \approx 5600$ Oe. Diese Tatsache deutet auf eine mikromagnetische Ursache der Widerstandsänderung hin.

Da sich der Hochfeldmagnetowiderstand im Vergleich zu Struktur 2 auch quantitativ nicht ändert (s. Abb. 7.17 b), kann der negative Magnetowiderstand in kleinen Feldern als unabhängig davon betrachtet werden. Es wird somit ein Prozess gesucht, welcher für hohe Magnetfelder einen weitgehend konstanten Widerstand verursacht, für kleine Felder aber eine Widerstandserniedrigung. Das spinpolarisierte Tunneln ist ein positiver Magnetowiderstand und kommt entsprechend nicht in Betracht. Auch der Anisotrope Magnetowiderstand kann nicht für dieses Verhalten verantwortlich sein, da für die angewandte Messgeometrie $(H \perp I)$ der Widerstand für hohe Felder $(H \| M)$ minimal sein sollte. Stellt weiterhin die Korngrenze die leichte Richtung der Magnetisierung dar, so sollte keine Widerstandsänderung auftreten, da die Magnetisierung immer (anti)parallel zum äußeren Feld liegt. Entsprechend gilt immer $M \perp I$, der Widerstand ist klein. Selbst wenn sich die Lage der leichten Richtung aufgrund der geänderten Formanisotropie ändert, kann der AMR das beobachtete Verhalten nicht erklären. Für kleine Felder würde $I \| M$ gelten, der Widerstand würde ansteigen, der AMR ist positiv.

Eine mögliche Erklärung liegt im so genannten Domänenwand-Magnetowiderstand (engl.: domainwall magnetoresistance, DWMR, siehe [31,222] sowie Zitate darin). Allerdings sind dessen mikroskopische Mechanismen Gegenstand der aktuellen Diskussion 31 , 222 und weitgehend ungeklärt. Verschiedene theoretische Berechnungen kommen sogar zu unterschiedlichen Vorzeichen des Magnetowiderstandes [31. Üblicherweise wird aber nur über kleine Werte des DWMR berichtet, s.d. dieser nur schwer von anderen Effekten $\mathrm{zu}$ trennen ist [223]. Wolfman et al. 224] hingegen berichten von ungewöhnlich hohen Effekten an LSMO-Nanokontakten, welche zusätzliche, durch Elektronenstrahl-Lithographie präparierte Gräben zur definierten Positionierung von Domänenwänden beinhalten. Für eine solche Geometrie beobachten die Autoren einen hohen, positiven MR. Wird die Struktur aber dahingehend modifiziert, dass die Gräben nicht den gesamten Stromstreifen queren, wie es in der Abbildung 7.22 dargestellt ist, so erhalten die Autoren bei Raumtemperatur einen hohen, negativen Magnetowiderstand [224], (Abbildung 7.22) (1). Dieser Magnetowiderstandsverlauf ist dem in dieser Arbeit unter Vernachlässigung des Hochfeld-MR beobachteten Verhalten qualitativ sehr ähnlich. Von hohen Feldern kommend verringert sich der Widerstand vor Erreichen des Nullfeldes, durchläuft ein Minimum der Breite $\Delta H \approx 2500$ Oe und springt auf seinen Hochfeldwiderstand zurück. Wie auch in dieser Arbeit ist der Feldwert der Widerstandserhöhung immer höher als derjenige der Widerstandsverringerung. Auch die Größenordnung der Widerstandsänderung stimmt gut überein (Wolfman et al.: ca. 14\%; diese Arbeit: 15-20\%). Mutmaßlich ist das Verhalten auf die gleiche mikroskopische Ursache zurückzuführen. Wolfman et al. 224 schließen auf einen DWMR mit einer mikromagnetischen Konfiguration im Nullfeld, welche für dieses

\footnotetext{
(1) Untersuchungen bei tieferen Temperaturen werden von den Autoren der Arbeit nicht erwähnt.
} 

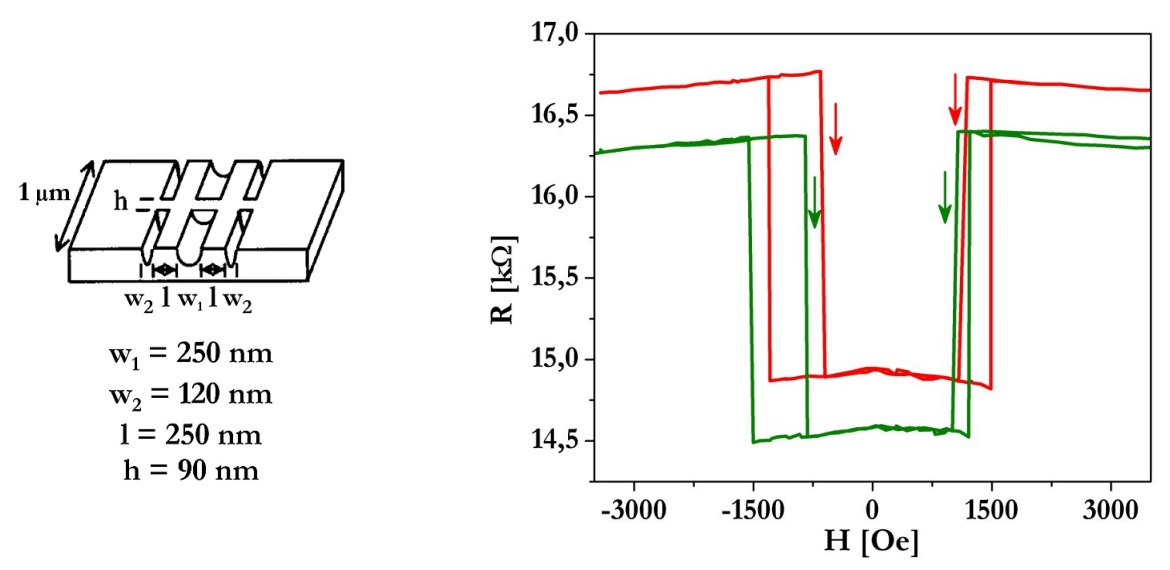

Abbildung 7.22. Ergebnisse nach Wolfman et al. 224. Links: Verwendete Geometrie des LSMO-Nanokontaktes. Lediglich die LSMO-Schicht $(d=80 \mathrm{~nm})$ ist dargestellt. Die durch Elektronenstrahl-Lithographie definierten Gräben sind ca. $40 \mathrm{~nm}$ tief und durchtrennen die Schicht entsprechend nicht vollständig. Rechts: Magnetowiderstand bei Raumtemperatur. Die überlappenden Kurven sind zur besseren Darstellung versetzt dargestellt. Nach [224].

überraschende Verhalten ursächlich ist. Sie konstatieren dabei, dass die genaue magnetische Konfiguration schwer zu antizipieren ist und eine direkte magnetische Bildgebung zur weiteren Aufklärung notwendig ist. In diesem Sinne muss die Ursache für das eher unübliche Magnetowiderstandsverhalten letztlich als ungeklärt angesehen werden.

\subsubsection{Strominduzierte Effekte}

Das strominduzierte Magnetisierungsschalten und die damit verbundenen Widerstandsänderungen in magnetoresistiven Elementen wurden bisher entweder an vertikal strukturierten Schichtsystemen oder mittels der Punktkontaktmethode an unstrukturierten Proben erfolgreich durchgeführt. Die in dieser Arbeit präparierten Bikristallkontakte stellen eine Möglichkeit dar, das strominduzierte Magnetisierungsschalten erstmalig auch in lateral strukturierten Systemen zu beobachten und gegebenenfalls zu charakterisieren. Die durchgeführten Experimente zeigen dabei zunächst, dass eine grundsätzliche Beeinflussung des Widerstandes durch den Strom gegeben ist (s. Abb. 7.18).

\section{LSMO:MgO Korngrenzen-Kontakte}

Der Widerstand des MgO-dotierten Korngrenzen-Kontaktes weist eine deutliche Stromabhängigkeit auf. Zwei Bereiche sind besonders von Widerstandsänderungen betroffen: Einerseits wird eine Abnahme des Widerstandes für kleine Stromstärken $|I| \leq 0,2 \mathrm{~mA}$ beobachtet, andererseits treten die oben beschriebenen Widerstandsänderungen bei ca. $\pm 0,5 \mathrm{~mA}$ auf. Eine Beschreibung der Strom-Spannungs-Kennlinie im Bereich $|U| \leq 2 \mathrm{~V}$ durch das Glazman-Matveev-Modell [121] gelingt wie für die Kennlinien von Struktur 2 gut, s.d. die Widerstandsabnahme für kleine Ströme auf einen Tunnelprozess über Zwischenzustände zurückgeführt werden kann. Entsprechend kann vermutet werden, dass der Transport von der dotierten Korngrenze dominiert wird. 
Für die abrupten Widerstandsänderungen bei hohen Strömen hingegen kann keine solide physikalische Ursache angegeben werden. Dabei bleibt zunächst festzuhalten, dass die Widerstandsänderungen sehr groß $\left(\Delta R / R_{\max }\right.$ bis zu $\left.50 \%\right)$ und reversibel sind. Ein Bereich, in dem zwei Widerstandszustände stabil sind, ist auf ein schmales Intervall der Breite $\Delta I \approx 0,1 \mathrm{~mA}$ um $I \approx-0,5 \mathrm{~mA}$ (je nach äußerem Magnetfeld) beschränkt und tritt nur für negative Stromstärken auf. Weiterhin wird der Widerstand durch den Strom erhöht, wodurch mögliche Mechanismen, welche auf einem Schmelzen einer gegebenenfalls vorhandenen ladungs- oder orbitalgeordneten Phase beruhen, ausgeschlossen werden können [14|. Zudem sollte für ein solches Szenario das äußere Magnetfeld die kritischen Stromstärken verringern [14], da der Einfluss des Stroms, respektive der Spannung, ebenso wie der des Magnetfeldes im Schmelzen der Ordnung bestehen. Dieses steht im Widerspruch zu den Beobachtungen.

Die Erhöhung des Widerstandes lässt auch das Widerstandsschalten durch Spannungspulse (EPIR, s. Kap. 2.6), welches vermutlich für die definierten Widerstandsänderungen im nanokristallinen LSMO verantwortlich ist (s. Kap. 6.3), als Ursache unwahrscheinlich erscheinen. Für einen EPIR-Mechanismus wird neben einer anfänglichen Widerstandsverringerung ein polaritätsabhängiger Widerstandszustand, bzw. die Abbildung 6.26 dargestellte Abhängigkeit des Widerstandes in symmetrischen Kontakten (s. Diskussion in Kap. 6.3 erwartet. Die experimentellen Ergebnisse weichen von diesen Vorhersagen deutlich ab. Auch eine Überlagerung der anfänglichen Widerstandsabnahmen mit der kontinuierliche Abnahme aufgrund des Tunneleffekts erscheint wenig wahrscheinlich, da diese Abnahme sehr gut durch das Glazman-Matveev-Modell beschrieben werden kann. Weiterhin zeigen die in Kapitel 6.3 erzeilten Ergebnisse, dass sich kein systematischer Einfluss des äußeren Feldes auf die kritischen Ströme/Spannungen zeigen sollte, was aber beobachtet wird. Dennoch bleibt festzuhalten, dass die kritischen Spannungen, bzw. elektrischen Felder, bei denen die Widerstandserhöhungen auftreten, größenordnungsmäßig mit denen eines EPIR-Mechanismus vergleichbar sind [87.

Weitere Mechanismen, welche das Auftreten der Widerstandsänderungen erklären können und insbesondere deren Symmetrie berücksichtigen, bestehen in Bandstruktureffekten sowie in Heizeffekten in Form von Joulescher Wärme. Andererseits macht die perfekte Symmetrie der Mechanismen sie wiederum auch unwahrscheinlich, da sie die für negative Ströme auftretende Hysterese, welche für positive Ströme absent ist, nicht erklären können. Die Magnetfeldabhängigkeit der kritischen Stromstärken/Spannungen spricht zudem gegen Bandstruktureffekte: Zwar ist eine Verschiebung von Energielücken o.ä. durch das äußere Feld vorstellbar, allerdings sind die magnetischen Energien vergleichsweise klein, s.d. eine Verschiebung der kritischen Spannungen um über $1 \mathrm{~V}$ nicht realistisch erscheint. Die scharfen Widerstandsänderungen sprechen auch gegen das Joulsche Heizen $\left(R \cdot I^{2}=2,5 \mathrm{~mW}\right.$ bei $0,5 \mathrm{~mA}$ und $\left.10 \mathrm{k} \Omega\right)$ als dominantem Prozess. Die Widerstandsänderung sollte aufgrund der kontinuierlichen Temperaturerhöhung ebenfalls kontinuierlich sein.

Schließlich soll das strominduzierte Magnetisierungsschalten als physikalische Ursache für die Widerstandsänderungen in Betracht gezogen werden. Dieser Mechanismus könnte zunächst die Widerstandszunahme erklären: In hohen äußeren Feldern ist die parallele 
Ausrichtungen der Magnetisierungen der Elektroden beidseits der Barriere stabil. Unter der Annahme eines positiven Magnetowiderstandes führt jede Abweichung von dieser Konfiguration zu einer Widerstandserhöhung. Da der Mechanismus magnetischer Natur ist, ist auch ein Einfluss eines äußeren Feldes auf die kritischen Stromstärken zu erwarten. Die Größenordnung der kritischen Stromdichten von $J_{C} \approx 2,5 \cdot 10^{6} \mathrm{~A} / \mathrm{cm}^{2}$ ist zwar im Vergleich mit auf metallischen Elektroden basierenden Systemen eher gering (beispielsweise $J_{C} \approx 10^{8} \mathrm{~A} / \mathrm{cm}^{2}$ in $\mathrm{Co} / \mathrm{Cu} / \mathrm{Co}$ Systemen $[122]$, andererseits berichtet Sun 126 von Widerstandsänderungen bei sehr viel geringeren Stromdichten und auch Pallecchi et al. 225] schildern hysteretische Maxima im differentiellen Widerstand für nur eine Stromrichtung bei $J_{C} \approx 10^{3}-10^{4} \mathrm{~A} / \mathrm{cm}^{2}$, welche sie auf strominduzierte Magnetisierungsanregungen zurückführen.

Wesentliche Merkmale der Widerstandsänderung können also durch das strominduzierte Magnetisierungsschalten beschrieben werden. Wiederum existieren mehrere Gegenargumente: Zum einen wird keine Abhängigekit des Widerstandes von der Polarität des Stromes beobachtet. Weiterhin sollte ohne eine Asymmetrie im System (unterschiedliche Koerzitivfelder der Elektroden, s. Kap. 3.3) ausschließlich die parallele Konfiguration der Magnetisierungen stabil sein. Zudem sind durch strominduziertes Magnetisierungsschalten verursachte Widerstandsänderungen immer mit der magnetfeldabhängigen Widerstandshysterese verknüpft. Hier zeigt sich aber das oben dikutierte ungewöhnliche Verhalten mit einem negativen Magnetowiderstand. Entsprechend sollte eine Widerstandsreduktion bei Erreichen der kritischen Stromdichte erwartet werden. Das Gegenteil ist der Fall.

Die am $\mathrm{CeO}_{2}$-dotierten FIB-Kontakt beobachteten Widerstandserhöhungen widersprechen den am MgO-dotierten Kontakt gefundenen Eigenschaften nicht, wobei aufgrund der Stabilitätsprobleme des Kontaktes keine weitere Charakterisierung vorgenommen werden konnte. Da aber auch der Magnetowiderstand ein qualitativ gleiches Verhalten aufweist, lässt sich auch für die strominduzierten Widerstandsänderungen ein allgemeinerer Mechanismus zumindest vermuten. 


\section{Kapitel 8}

\section{Zusammenführende Diskussion}

In diesem Kapitel werden die in den beiden vorangegangenen Kapiteln vorgestellten Ergebnisse in einem gemeinsamen Kontext diskutiert. Dies betrifft im Speziellen das Auftreten eines hohen Magnetowiderstandseffektes, die Frage nach einem gestörten Bereich des Manganats an der Korngrenze sowie schließlich die Ursache der strom- bzw. spannungsinduzierten Widerstandseffekte. Dabei müssen die Unterschiede zwischen den Nanokompositen und den Bikristall-Kontakten detailliert berücksichtigt werden. Diese sind zunächst struktureller Natur.

\subsection{Struktur}

Ein wesentlicher Unterschied zwischen den Nanokompositen und den Bikristall-Kontakten ist die Kristallorientierung bezüglich des Substrats. Während in den Bikristall-Kontakten aufgrund des epitaktischen, (001)-orientierten Wachstums nur eine wohldefinierte Korngrenze mit bekannter tilt-Komponente auftritt, ist zwar für die Nanokomposite eine (111)Textur zu beobachten, innerhalb der Schichtebene hingegen zeigt sich keine Vorzugsorientierung. Hieraus ergeben sich zunächst Korngrenzen mit reinen tilt-Komponenten, welche aber nicht zwingend symmetrisch sind. Über die Winkel kann keine Aussage getroffen werden. Zusätzlich existiert eine geringere Anzahl von Körnern anderer Orientierung bezüglich des Substrats, wodurch auch Korngrenzen mit twist-Komponenten auftreten.

Neben der Orientierung der Korngrenzen muss auch der Spannungszustand des Manganats berücksichtigt werden. Hier ist insbesondere die Wahl des Substrats, aber auch der Einfluss der zweiten chemischen Phase zu beachten. Das nanokristalline LSMO und die Nanokomposite sind weitgehend unverspannt, wohingegen die Deposition auf STOBikristall-Substraten erwartungsgemäß zu verspannten Filmen führt. Neben einem Einfluss auf die Stärke der Austauschmechanismen ist eine Beeinflussung der magnetischen Anisotropien zu berücksichtigen [213, 214|. Ebenso sollte der elektronische Charakter der Korngrenze sensitiv auf eine Änderung des Spannungszustandes reagieren, da die isolierenden Eigenschaften der Korngrenzen häufig auf den Einfluss mechanischer Spannungen und Unordnung zurückgeführt werden 28.

Die erzielten Ergebnisse zeigen, dass auch die Dotierung mit einer zweiten chemischen 
Phase den Spannungszustand des Manganats beeinflussen kann. Hierbei kommt es vermutlich zu einer komplexen Wechselwirkung der Spannungsfelder, welche einerseits durch das Substrat und andererseits durch die zweite Phase hervorgerufen werden. So zeigt sich für die Nanokomposite ein im Wesentlichen ungeänderter Gitterparameter des LSMO bei Dotierung mit $\mathrm{CeO}_{2}$, dessen Gitterparameter seinerseits aufgrund der La-Dotierung eine Änderung aufweist. Bei Deposition auf STO ist das Gegenteil zu beobachten: Der ohnehin gegenüber dem Volumenwert verringerte $c$-Achsen-Gitterparameter des undotierten LSMO von $c=3,854 \AA$ nimmt weiter ab auf $c=3,851 \AA$, wohingegen der des $\mathrm{CeO}_{2}$ gegenüber dem Volumenwert unverändert bleibt. Die mikroskopische Ursache für dieses Verhalten bleibt dabei offen.

Über die Verteilung der isolierenden Phase in der Korngrenze der dotierten BikristallKontakte kann keine genaue Aussage getroffen werden (s. Kap. 7.4.2 und Abb. 7.21). Daher ist der Einfluss dieser Phase unter Umständen anders zu bewerten als für die Nanokomposite, in denen zumindest im System LSMO:MgO eine klare Trennung der einzelnen Körnern durch das $\mathrm{MgO}$ mittels TEM-Untersuchungen verifiziert wurde [29].

Ein weiteres Unterscheidungsmerkmal liegt im Einfluss der Strukturgröße. So wurden für die Bikristalle strukturelle (und daraus resultierend elektronische) Inhomogenitäten beobachtet (s. Kap. 7.4.2), während insbesondere die Untersuchung der $\mathrm{CeO}_{2}$-dotierten Nanokomposite eine nur geringe Variation der Transporteigenschaften mit abnehmender Strukturgröße ergeben hat (s. Kap. 6.2.4).

\subsection{Magnetotransport}

Trotz der strukturellen Unterschiede zwischen den Nanokompositen und BikristallKontakten werden gemeinsame Muster im elektronischen Verhalten beobachtet. Dies betrifft sowohl den Magnetowiderstand (s.u.) als auch die Schulter, bzw. das Maximum im temperaturabhängigen Widerstandsverlauf. Letzteres wird in Verbindung mit der unterschiedlichen Temperaturabhängigkeit der Magnetisierung und des LFMR häufig als Indiz für einen gestörten Bereich des Manganats an der Korngrenze, bzw. an der Grenzfläche zur isolierenden Phase gewertet [76, 31]. Merkmale eines gestörten Bereichs sind in allen untersuchten Systemen zu beobachten. So zeigt sich im nanokristallinen LSMO die Bedeutung eines isolierenden Bereiches in der Möglichkeit des spannungspulsgetriebenen Widerstandsschaltens. Andererseits ergeben sich aus den mittels niederfrequenter $1 / f$-Rauschspektroskopie am MgO-dotierten Nanokomposit erzielten Ergebnissen keine Hinweise auf einen solchen Bereich. Die Temperaturabhängigkeit der normierten Rauschamplitude lässt sich zumindest im zugänglichen Temperaturintervall gut durch einen auf dem Tunnelprozess basierenden Mechanismus beschreiben und benötigt keine weiteren Parameter. Auch der Absolutwert der Rauschamplitude ist sehr gering und vergleichbar mit Werten für epitaktische Proben (s. Kap. 6.1.4). Gegebenenfalls kann eine solche Diskrepanz der Beobachtungen durch die Beschränkung der Rauschanalyse auf $T \leq 100 \mathrm{~K}$ erklärt werden. In diesem Temperaturbereich sind im qualitativen Verlauf des Widerstandes, abgesehen von mutmaßlichen Coulomb-Blockade-Effekten bei sehr 
tiefen Temperaturen, keine signifikanten Abweichungen vom Verhalten eines Einkristalls zu beobachten. Die zusätzliche Schulter, bzw. das Maximum befindet sich üblicherweise in einem Temperaturbereich von ca. 150-250 K und ist mittels der Rauschspektroskopie unter den gegebenen Bedingungen nicht zugänglich. Möglicherweise treten signifikante Störungen des Manganats an der Grenzfläche erst oberhalb von $T=100 \mathrm{~K}$ auf und haben daher keinen Einfluss auf die Ergebnisse der Rauschspektroskopie. Dies würde die hohen Magnetowiderstandswerte von bis zu 235\% bei tiefen Temperaturen (s. Kap. 7.2.1) plausibel machen. Gegen ein solches Szenario eines ungestörten Bereichs unterhalb von $T=100 \mathrm{~K}$ sprechen hingegen die spannungsinduzierten Widerstandsänderungen im nanokristallinen LSMO bei $T=5 \mathrm{~K}$ (s. Kap. 6.3.4), welche letztlich mit den Korngrenzen in Verbindung gebracht werden konnten. Hier wird der Einfluss eines gestörten Bereichs zumindest vermutet. Andererseits muss beim Vergleich der Systeme ein mögliche Änderung der Grenzflächeneigenschaften durch das MgO berücksichtigt werden (s.o.). Genaueren Aufschluss würden Rauschanalysen im Temperaturbereich der auftretenden Schulter, bzw. des Maximums liefern.

Weiterhin ist sowohl den nanokristallinen Systemen als auch den Bikristall-Kontakten der ausgeprägte Kleinfeld-Magnetowiderstandseffekt zu Eigen. In den Nanokompositen, bzw. dem nanokristallinen System werden Maximalwerte von ca. 15\% (undotiert), 30\% (MgO-dotiert) und $60 \%\left(\mathrm{CeO}_{2}\right.$-dotiert) ermittelt, wohingegen die Widerstandsänderungen in den Bikristall-Kontakten mit bis zu 235\% (undotiert) deutlich höher ausfallen. Dabei wird neben einem Unterschied in der Strukturgrößenabhängigkeit offenbar, dass die Form der Widerstandshysterese unterschiedlich ist. Während die Widerstandsänderungen in den nanokristallinen Systemen ausschließlich kontinuierlich sind, werden in den Bikristall-Kontakten je nach Strukturgröße kontinuierliche oder sprunghafte Änderungen beobachtet. Das Verhalten der Bikristall-Kontakte liegt dabei vermutlich in mikromagnetischen Eigenschaften, vornehmlich unterschiedlichen Anisotropiebeiträgen, begründet. Offensichtlich können diese Charakteristika nicht ohne weiteres auf die nanokristallinen Systeme übertragen werden, da die Widerstandsänderungen deutlich geringer ausfallen. Hierfür können mehrere Gründe verantwortlich sein. Eine erste Erklärung liegt in unterschiedlichen magnetischen Anisotropien. Es ist beispielsweise vorstellbar, dass in den nanokristallinen Systemen keine antiparallele Orientierung der magnetischen Momente erreicht wird, wodurch die Widerstandsänderungen geringer ausfallen. So stimmen die Widerstandsmaxima als Funktion des äußeren Feldes mit den Koerzitivfeldern der Proben überein, an denen eine statistische Verteilung der magnetischen Momente wahrscheinlicher ist als eine antiparallele Orientierung. In den Bikristall-Systemen wird die antiparallele Konfiguration vermutlich aufgrund der geänderten Anisotropie erreicht. In den nanokristallinen Systemen hingegen ist die mikromagnetische Konfiguration a priori nicht eindeutig. Zwar kann eine leichte Magnetisierungsachse parallel zur Schichtnormalen aufgrund von Hysteresemessungen ausgeschlossen werden (das Aspektverhältnis der einzelnen Säulen ist offenbar nicht groß genug, um die Formanisotropie der gesamten Schicht zu überwinden), dennoch ist die Domänenkonfiguration innerhalb der Schichtebene nicht geklärt. Dabei ist zusätzlich zu beachten, dass aufgrund der Mikrostruktur der Winkel zwischen äußerem Feld und den Korngrenzen variiert. 
Neben einer aufgrund der Mikrostruktur geänderten magnetischen Anisotropie in den unterschiedlichen Systemen ist für die nanoskaligen Materialien eine Verteilung der Koerzitivfelder zu berücksichtigen. Selbst wenn zwischen allen benachbarten Körnern eine antiparallele Konfiguration erzielt werden könnte, würde aufgrund einer Verteilung der Koerzitivfelder, welche beispielsweise durch eine Verteilung der Korngröße induziert werden könnte, die Widerstandsänderung insgesamt geringer ausfallen und kontinuierlich sein.

Gegebenenfalls erfordert ein Vergleich der an den unterschiedlichen Systemen erzielten Ergebnisse auch eine Berücksichtigung der Symmetrien der Elektronenwellen. Wie beispielsweise die Berechnungen von Butler et al. [111 für das System Fe/MgO/Fe gezeigt haben (s.a. Kap. 3.2.2), ist eine genaue Betrachtung der Bandstrukturen sowohl der Elektroden als auch der Barriere von großer Bedeutung. Dies könnte auch für den Kleinfeld-Magnetowiderstand in Manganaten relevant sein, wobei insbesondere die relative Orientierung der Körner essentiell ist. Da im Wesentlichen nur Elektronen tunneln, die nahezu senkrecht auf die Potentialschwelle zulaufen (s. Kap. 3.1.2), bleibt die Richtung des tunnelnden Elektrons bezüglich des Gitters bei einer symmetrischen Korngrenze, also für die Bikristall-Kontakte, erhalten. Da in den nanokristallinen Systemen die Orientierung der Säulen aber innerhalb der Schichtebene statistisch verteilt ist, sind keine symmetrischen Korngrenzen zu erwarten. Speziell in Systemen mit anisotropen Fermiflächen ist entsprechend mit einem Einfluss der Bandstruktur auf den Tunnelprozess und somit den Magnetowiderstand zu rechnen. Hinweise auf die Bedeutung der strukturellen Kohärenz innerhalb der Schichtebene werden auch von Gu et al. gefunden [226]. Neben der relativen Orientierung der Körner ist unter Umständen die unterschiedliche Orientierung im $k$-Raum der vornehmlich tunnelnden Elektronen innerhalb der Schichtebene zu berücksichtigen. Aufgrund der (001)-Epitaxie der Bikristalle und einer (111)-Textur für die Nanokomposite sollten die tunnelnden Elektronen unterschiedliche Richtungen im $k$-Raum aufweisen.

Die obigen Erläuterungen zeigen, dass die Ergebnisse der Bikristall-Kontakte zumindest in Hinblick auf das Magnetowiderstandsverhalten nicht ohne Weiteres auf die nanoskaligen Systeme übertragen werden können.

In Zusammenhang mit Bandstruktur-Effekten sollen auch die voneinander abweichenden, maximalen Magnetowiderstandsänderungen der verschiedentlich dotierten, nanokristallinen Systeme diskutiert werden. Zunächst ist festzuhalten, dass die Dotierung der Korngrenzen sowohl mit $\mathrm{MgO}$ als auch mit $\mathrm{CeO}_{2}$ zu einer deutlichen Erhöhung des LFMR gegenüber dem undotierten Fall führt, wie es auch in der Literatur beobachtet wird 29,30. Dies wird üblicherweise auf eine magnetische Entkopplung der einzelnen Körner zurückgeführt [30]. Dennoch sind bei Dotierung mit verschiedenen Materialien unterschiedliche Magnetowiderstandswerte festzustellen. Wie in Kapitel 2.5.2 beschrieben, weisen $\mathrm{MgO}$ und $\mathrm{CeO}_{2}$ sowohl unterschiedliche Bandlücken als auch Gitterparameter auf. Es zeigt sich, dass sich bei Verwendung von $\mathrm{CeO}_{2}$ eine fast zu vernachlässigende Gitterfehlpassung ergibt, die wiederum für $\mathrm{MgO}$ vergleichsweise hoch ist (s. Kap. 2.5.2). Die Verwendung von $\mathrm{MgO}-$ Barrieren in Verbindung mit Fe-Elektroden basiert gleichermaßen vorwiegend auf strukturellen Überlegungen, da ebenfalls in (001)-Richtung unter einer $45^{\circ}$-Rotation eine geringe Gitterfehlpassung herrscht $\left(c_{M g O}=4,2 \AA, c_{F e} \cdot \sqrt{2}=4,1 \AA\right)$. Wenige Atomlagen 
MgO können daher pseudomorph auf (001)-orientiertem Fe deponiert werden [111]. Wie die experimentellen Ergebnisse von Yuasa et al. [4] sowie Parkin et al. [5] gezeigt haben, ist diese strukturelle Kohärenz fundamental für das Auftreten von Bandstruktur-Effekten. Womöglich ist der vergleichsweise hohe LFMR des $\mathrm{CeO}_{2}$-dotierten Systems ebenfalls auf eine solche strukturelle Kohärenz in Verbindung mit Bandstruktur-Effekten zurückzuführen. $\mathrm{Zu}$ beachten ist dabei jedoch die in dieser Arbeit beobachtete La-Dotierung der $\mathrm{CeO}_{2}$-Phase, deren Einfluss auf die Bandstruktur unbekannt ist.

\subsection{Strom-/Spannungsinduzierte Effekte}

Die an unterschiedlichen Systemen beobachteten strom- bzw. spannungsinduzierten Effekte führen zu keinem einheitlichen Bild. Es treten verschiedene Verhaltensmuster auf: Zunächst sei auf die massiven Widerstandsänderungen im nanokristallinen LSMO hingewiesen, welche an ca. $1 \mu \mathrm{m}$ langen Mikrostrukturen unterschiedlicher Breite beobachtet werden. Diese vermutlich durch das elektrische Feld induzierten, abrupten Widerstandsvariationen von bis zu 700\% werden in solcher Deutlichkeit in keinem der weiter untersuchten Systeme gefunden.

Ein Grund könnte in der durch den geringen Abstand der Kontaktflächen erhöhten elektrischen Feldstärke liegen. Für die Untersuchung weiterer Systeme werden die ehedem langen Strukturen mittels FIB ausgedünnt und der Abstand der Kontaktflächen durch Pt-Deposition im FIB verringert. Dabei ist einerseits zu beachten, dass der spezifische Widerstand des im FIB deponierten Pt deutlich gegenüber konventionell deponiertem Pt erhöht ist ${ }^{(1)}$, anderseits können Beschädigungen des Kontaktes durch den Ionenstrahl nicht ausgeschlossen werden 228. Die hochohmigen Zuleitungen könnten zu einer Verringerung der effektiven Feldstärke führen, s.d. die kritische Feldstärke nicht erreicht wird und entsprechende Effekte nicht auftreten. Andererseits fallen die Spannungen im Manganat im Wesentlichen an den Korngrenzen ab. Bei gleicher Gesamtspannung wäre in den nanoskaligen Systemen entsprechend der erhöhten Anzahl der Korngrenzen das elektrische Feld über eine Korngrenze hinweg geringer als in einem Bikristall-Kontakt. Stellte also das elektrische Feld über eine Korngrenze hinweg das alleinige Kriterium zur Beobachtung der Widerstandsänderungen dar, so sollte es auch in den FIB-Kontakten zu beobachten sein.

Auch wenn mögliche Beschädigungen durch die FIB-Präparation nicht ausgeschlossen werden können, zeigen die Bikristallkontakte dennoch ein reproduzierbares magnetoresistives Verhalten, welches ebenfalls in der Literatur beobachtet wird 224 (s. Kap. 7.4.2). Diese Tatsache lässt Beschädigungen unwahrscheinlicher erscheinen, da diese anderernfalls sehr systematisch aufträten. Entsprechend kann ein Einfluss auf das Schaltverhalten zwar nicht ausgeschlossen, muss aber mit gebührender Vorsicht bewertet werden.

Ein weiterer Grund für die Beschränkung des EPIR-Mechanismus auf das nanokristalline LSMO könnte abermals in der Modifikation des Korngrenzenbereichs durch die zweite

\footnotetext{
${ }^{(1)}$ Ein wesentlicher Grund liegt in Resten des organischen Trägermaterials, welches ebenfalls deponiert wird 227 .
} 
Phase liegen. Gegebenfalls kommt es durch die zweite Phase zu einer Änderung des Spannungszustandes des Manganats in der Nähe der Grenzfläche, wodurch unter Annahme des für das nanokristalline LSMO entwickelten, idealisierten Strukturmodells ein Einfluss auf das Schaltverhalten resultiert. Möglicherweise sind die vorauszusetzenden Modifikationen im geänderten Spannungszustand nicht weiter stabil. Letztlich sind weiterführende Untersuchungen nötig, besonders eine hochauflösende Strukturbestimmung an der Korngrenze, um die mikroskopischen Mechanismen und die Unterschiede zwischen den in dieser Arbeit untersuchten Systemen zu klären.

Auch die in den anderen Systemen beobachteten Widerstandsänderungen zeigen kein einheitliches Bild. Während im FIB-Kontakt der nanoskaligen LSMO:CeO $\mathrm{C}_{2}$-Probe (s. Abb. 6.13) nur vergleichsweise geringe Widerstandsänderungen auftreten, sind diese im MgOdotierten Bikristallkontakt sehr deutlich (s. Abb. 7.18). In beiden Fällen kann jedoch keine solide physikalische Erklärung der Phänomene gegeben werden. Dennoch ist auffällig, dass die beiden Systeme unterschiedliche magnetoresistive Eigenschaften aufweisen. Dies kann als Hinweis darauf gedeutet werden, dass das strominduzierte Verhalten an die magnetoresistiven Merkmale gekoppelt ist. Diese These wird gestützt durch die partielle Magnetfeldabhängigkeit der kritischen Größen, welche für das spannungsinduzierte Widerstandsschalten im nanokristallinen LSMO nicht beobachtet wird. Entsprechend sind Prozesse, welche auf dem strominduzierten Magnetisierungsschalten basieren, gegenüber anderen Mechanismen als wahrscheinlicher anzusehen, was bereits in den Diskussionen der einzelnen Phänomene angedeutet wurde.

Auch wenn die genauen physikalischen Mechanismen der Widerstandsänderungen im Detail ungeklärt sind, bleibt festzuhalten, dass neben den massiven, spannungsinduzierten Widerstandsänderungen im nanoskaligen LSMO weitere, reproduzierbare Widerstandsmodifikationen in lateral strukturierten Systemen gefunden werden konnten. Weitere strukturelle und elektronische Charakterisierungen, sowie eine Stabilisierung insbesondere der Bikristallkontakte sind dabei notwendig, um die mikroskopischen Prozesse genauer zu identifizieren und gegeneinander abzugrenzen. 


\section{Kapitel 9}

\section{Zusammenfassung und Ausblick}

\subsection{Zusammenfassung}

In der vorliegenden Arbeit wurde der Einfluss von Grenzflächen auf das Transportverhalten von LSMO untersucht, sowie diese Grenzflächen im Detail charakterisiert. Hierzu wurden systematische Untersuchungen unter besonderer Berücksichtigung selbstorganisierter Prozesse und einzelner Korngrenzen durchgeführt. In fortführenden Analysen wurde weiterhin gezeigt, dass durch gezielte Berücksichtigung der Grenzflächen das Verhalten in Form von reversiblen Widerstandsänderungen bewusst beeinflusst werden kann.

Mittels MAD wurden sowohl undotierte als auch mit $\mathrm{MgO}$ - oder $\mathrm{CeO}_{2}$-dotierte LSMOSchichten auf unterschiedlichen Substraten präpariert, wodurch die Mikrostruktur der Proben gezielt kontrolliert werden kann. Die Deposition auf $\mathrm{Al}_{2} \mathrm{O}_{3}$ führt zu einem polykristallinen Wachstum mit einer (111)-Textur des LSMO und einer mittleren Korngröße von ca. $50 \mathrm{~nm}$. Auf (001)-orientierten STO- und MgO-Bikristallen präparierte Schichten weisen hingegen aufgrund des epitaktischen Wachstums auf den beiden Halbkristallen eine definierte Korngrenze auf. In beiden Fällen wurde die Modifikation der Korngrenzen durch eine zweite, isolierende Phase untersucht, welche in der Korngrenze segregiert.

\section{Bikristallkontakte}

Die Charakterisierung der Bikristallkontakte wird wesentlich durch die mangelhafte Qualität der Substrate beeinflusst. TEM- und REM-Analysen zeigen Löcher in der künstlichen Korngrenze des Substrates, welche sich aufgrund des epitaktischen Wachstums auf die Schicht übertragen. Der Transportmechanismus über die Korngrenze hinweg zeigt eine starke Abhängigkeit von der Breite des Kontaktes, was auf strukturelle Inhomogenitäten in der Korngrenze zurückgeführt werden konnte. Für die vergleichsweise breiten Kontakte $(d=50 \mu \mathrm{m})$ ergibt sich ein Magnetowiderstandsverhalten, welches auf die Rotation der magnetischen Momente beidseitig der Korngrenze zurückgeführt werden konnte. Dabei zeigte sich, dass der Spannungszustand, welcher über die Wahl des Substrats beeinflussbar ist, einen zusätzlichen Parameter darstellt. Die erzielten Magnetowiderstandswerte sind gering, was sich jedoch durch die Variation der Kontaktbreite ändert. So wird beispielsweise für den undotierten Kontakt, deponiert auf einem STO-Bikristall, ein Magnetowi- 
derstand von über $230 \%$ bei einer Kontaktbreite von $d \approx 3 \mu \mathrm{m}$ bestimmt, während er für $d=50 \mu \mathrm{m}$ lediglich 5,5\% beträgt. Auch weitere Transporteigenschaften ändern sich deutlich: Der spezifische Widerstand steigt stark an, die Strom-Spannungs-Kennlinien sind nichtlinear und der Magnetowiderstand wird dominiert durch eine uniaxiale Anisotropie mit der Korngrenze als leichte Richtung. Der Transportmechanismus kann auf einen mehrstufigen Tunnelprozess über Defektzustände in der Barriere zurückgeführt werden. Als geeigneter Kontrollparameter dient der Widerstand des Kontaktes. Ein geändertes Transportverhalten (hoher MR, nichtlineare Strom-Spannungs-Kennlinie) bei Verringerung der Kontaktbreite stellt sich immer dann ein, wenn der spezifische Widerstand eine deutliche Erhöhung aufweist, was nicht für jeden Kontakt der Fall ist. Anderenfalls treten kaum Veränderungen gegenüber dem Verhalten des breiten Kontaktes auf. Unabhängig von der Kontaktbreite ist jedoch das Auftreten eines Maximums in der Temperaturabhängigkeit des maximalen Magnetowiderstands. Dies wird als Hinweis auf eine spinglasartige, magnetische Ordnung innerhalb der Korngrenze angesehen.

Der Einfluss der Dotierung mit einer zweiten Phase auf das Transportverhalten ist gering. Die erzielten Ergebnisse zeigen, dass die zweite Phase mutmaßlich in der Korngrenze angehäuft ist, die beiden Halbkristalle jedoch nicht vollständig separiert. Dies ist vermutlich auf einen zu geringen Anteil der zweiten Phase zurückzuführen.

Mittels FIB wurden Korngrenzenkontakte weiter ausgedünnt, was zu ungewöhnlichen, aber reproduzierbaren, magnetoresistiven und strominduzierten Effekten führte. Ein hoher, negativer Magnetowiderstand in kleinen Feldern, welcher gegebenenfalls auf einen Domänenwandmagnetowiderstandseffekt zurückzuführen ist, dominiert das magnetoresistive Verhalten. Weiterhin konnten massive, reversible Widerstandsänderungen unter Einfluss eines elektrischen Stromes bzw. eines elektrischen Feldes in lateral strukturierten Systemen hervorgerufen werden.

\section{Nanokomposite}

Mittels niederfrequenter 1/f-Rauschspektroskopie konnte die hohe Qualität der nanoskaligen LSMO:MgO-Schichten und insbesondere deren Grenzflächen nachgewiesen werden. Die Temperaturabhängigkeit der normierten Rauschamplitude konnte durch einen Tunneleffekt erklärt werden, welcher demnach als dominante Ursache des Rauschens angesehen werden kann. Die Größenordnung der normierten Rauschamplitude ist vergleichbar mit der von epitaktischen Manganatschichten. Da üblicherweise Defekte wie Korngrenzen und Grenzflächen als Ursache für das niederfrequente Rauschen angesehen werden, kann so auf eine hohe Qualität und wohldefinierte Strukturen geschlossen werden. Insbesondere haben die im zugänglichen Temperaturbereich von $T \leq 100 \mathrm{~K}$ systematisch durchgeführten Analysen keinen Hinweis auf einen gestörten Bereich des Manganats an der Grenzfläche zum Isolator ergeben.

Der im Vergleich zu dem MgO-dotierten System erhöhte Magnetowiderstand des $\mathrm{CeO}_{2^{-}}$ dotierten Systems von bis zu 60\% ist vermutlich auf die geringere Barrierenhöhe und auf die Gitterpassung an das LSMO zurückzuführen. Dennoch konnte gezeigt werden, dass es zu einer mit der $\mathrm{CeO}_{2}$-Dotierung $x$ ansteigenden La-Anreicherung des $\mathrm{CeO}_{2}$ kommt. 
Mit Hilfe eines core-shell-Modells konnte im Weiteren die Diskrepanz zwischen der CurieTemperatur und der Temperatur des Metall-Isolator-Übergangs sowie das als Funktion der Dotierung $x$ auftretende Maximum im Magnetowiderstand erklärt werden. Durch eine systematische Verringerung der Anzahl der in den Transportprozess involvierten Körner konnte zudem nachgewiesen werden, dass bei tiefen Temperaturen die ermittelten, globalen Eigenschaften intrisch sind für jede einzelne Grenzfläche. Schließlich wurden temporäre, strominduzierte Widerstandsänderung bei Stromdichten von $J_{C} \approx 1,5 \cdot 10^{5} \mathrm{~A} / \mathrm{cm}^{2}$ nachgewiesen.

Im nanokristallinen LSMO wurden schließlich spannungsinduzierte, reversible Widerstandsänderung gefunden und charakterisiert. Nach Überschreiten einer kritischen Stromstärke definierter Polarität wird ein neuer, niederohmiger Zustand beobachtet, welcher durch Anwendung einer bestimmten Stromstärke gegensätzlicher Polarität wieder in den hochohmigen Ausgangszustand überführt werden kann. Die Widerstandsänderungen betragen bis zu 700\%. Aufgrund des hysteretischen Verhaltens sind in einem gewissen Bereich beide Zustände stabil.

Anhand systematischer Untersuchungen und Symmetrieüberlegungen konnte gezeigt werden, dass dieses anfänglich gefundene, asymmetrische Widerstandsverhalten auf die angewendete Messvorschrift zurückzuführen ist. Hierdurch konnte das elektrische Feld als ursächlich für die Widerstandsmodifikationen nachgewiesen werden. Weiterhin haben Vergleichsexperimente an epitaktischen Proben die Notwendigkeit der Korngrenzen für das Widerstandsschalten aufgezeigt. Zudem konnte so ein möglicher Kontaktwiderstand als Ursache weitgehend ausgeschlossen werden. Auf Grundlage der experimentellen Beobachtungen wurde schließlich ein idealisiertes Strukturmodell zur Beschreibung der Widerstandsänderungen entwickelt. Es basiert auf der Annahme einer strukturellen Modifikation der Korngrenze und einer damit verbundenen Änderung der Barriereneigenschaften. Hierzu werden zwei idealisierte, isostrukturelle Konfigurationen des Manganats angenommen, welche den Grundzustand in der Nähe der Korngrenze bilden. Weiterhin wird angenommen, dass eine aufgrund der größeren Bindungswinkel leitfähigere Konfiguration, welche dem Korninneren ähnlich ist, ein lokales Minimum in der verallgemeinerten Potentiallandschaft der Korngrenze aufweist. Ein elektrisches Feld führt zur Transformation zwischen den beiden isostrukturellen Modifikationen, welche zwangsläufig über die metastabile Modifikation führt, wodurch die Barriereneigenschaften und folglich die resistiven Eigenschaften geändert werden.

\section{Fazit}

Die Arbeit hat gezeigt, dass in LSMO durch systematische Charakterisierung und darauf basierende, gezielte Änderungen der Grenzflächeneigenschaften Einfluss auf das Transportverhalten genommen werden kann. Dies gelingt durch die Modifikation der Korngrenzen einerseits durch Hinzufügen einer weiteren Phase, andererseits durch ein ausreichend hohes elektrisches Feld. Wie für nanokristallines LSMO gezeigt werden konnte, können auf diese Weise beispielsweise der Kleinfeldmagnetowiderstand und der EPIR miteinander verbunden werden, wodurch sich neue Wege in der Nanotechnologie eröffnen. 


\subsection{Ausblick}

Mit dieser Arbeit eröffnen sich besonders durch das spannungsinduzierte Widerstandsschalten in nanokristallinem LSMO weitere, ungelöste Schwierigkeiten. Eine hochauflösende TEM-Analyse der Korngrenzen auch unter Einfluss eines elektrischen Feldes könnte das idealisierte Strukturmodell konkretisieren oder auch negieren. Der Einfluss der Mikrostrukturierung ist ebenfalls unklar. Eine systematische Variation der Stegbreite und -länge ebenso wie der Korngröße könnte weitere Aufschlüsse über die mikroskopischen Mechanismen ergeben. Zusätzlich bietet der weit zugängliche Temperaturbereich die Möglichkeit, thermische Effekte auf das Widerstandsschalten zu analysieren.

Erste Untersuchungen zur weiteren mikroskopischen Charakterisierung wurden bereits durchgeführt. Hierzu eignen sich die präparierten Bikristallkontakte, welche aber zur Untersuchung der spannungsinduzierten Widerstandsänderungen mit höherer Lebensdauer strukturiert werden müssen. Dennoch konnten mittels Rastertunnelmikroskopie (engl.: scanning tunneling microscope, STM) und ortsaufgelöster Leitfähigkeitsmessungen (engl.: scanning tunneling spectroscopy, STS) in der Nähe der Korngrenze reversible Leitfähigkeitsänderungen beobachtet werden [229]. Dabei erzeugt ein STM hohe lokale elektrische Felder in der Größenordnung von $10^{7}-10^{9} \mathrm{~V} / \mathrm{m}$. Diese Methode stellt entsprechend ein geeignetes Mittel zur lokalen Analyse der Widerstandsänderungen dar, welches sowohl für die Bikristalle als auch für das nanokristalline System zur weiteren Charakterisierung genutzt werden kann.

Abschließend sei angemerkt, dass insbesondere die Verknüpfung verschiedener resistiver Effekte in Manganaten auch in Zukunft ein spannendes Forschungsfeld sein wird und gegebenfalls auch zum weiteren Verständnis der grundlegenden Fragestellungen in diesen hochkomplexen Materialien beitragen kann. 


\section{Literatur}

[1] G.A. Prinz, „Magnetoelectronics“, Science 282, 1660 (1998)

[2] M. Jullière, „Tunneling between ferromagnetic films“, Phys. Lett. 54A, 225 (1975)

[3] J.S. Moodera, L.R. Kinder, T.W. Wong and R. Meservey, „Large magnetoresistance at room temperature in ferromagnetic thin film tunnel junctions", Phys. Rev. Lett. 74, 3273 (1995)

[4] S. Yuasa, T. Nagahama, A. Fukushima, Y. Suzuki und K. Ando, „Giant roomtemperature magnetoresistance in single-crystal $\mathrm{Fe} / \mathrm{MgO} / \mathrm{Fe}$ magnetic tunnel junctions", Nat. Mater. 3, 868 (2004)

[5] S.S.P. Parkin, C. Kaiser, A. Panchula, P.M. Rice, B. Hughes, M. Samant und S.-H. Yang, „Giant tunneling magnetoresistance at room temperature with $\mathrm{MgO}(100)$ tunnel barriers", Nat. Mater. 3, 862 (2004)

[6] J.C. Slonczewski, „Current-driven excitation of magnetic multilayers“, J. Magn. Magn. Mater. 159, L1 (1996)

[7] L. Berger, „Emission of spin waves by a magnetic multilayer traversed by a current“, Phys. Rev. B 54, 9353 (1996)

[8] M. Tsoi, A.G.M. Jansen, J. Bass, W.-C. Chiang, M. Seck, V. Tsoi und P. Wyder, „Excitation of a Magnetic Multilayer by an Electric Current", Phys. Rev. Lett. 80, 4281 (1998)

[9] E.B. Myers, D.C. Ralph, J.A. Katine, R.N. Louie und R.A. Buhrman „CurrentInduced Switching of Domains in Magnetic Multilayer Devices“, Science 285, 867 (1999)

[10] J.-H. Park, E. Vescovo, H.-J. Kim, C. Kwon, R. Ramesh und T. Venkatesan, „Direct evidence for a half-metallic ferromagnet", Nature 392, 794 (1998)

[11] E. Dagotto, „Complexity in strongly correlated electronic systems“, Science 309, $257(2005)$

[12] Y. Tokura, „Fundamental features of colossal magnetoresistive manganese oxides“, In Y. Tokura, editor, Advances in condensed matter science, Vol. 2: Colossal magnetoresistive oxides, S. 1-52, Gordon and Breach Science Publishers, 2000

[13] R. von Helmolt, J. Wecker, B. Holzapfel, L. Schultz und K. Samwer, „Giant negative magnetoresistance in perovskitelike $\mathrm{La}_{2 / 3} \mathrm{Ba}_{1 / 3} \mathrm{MnO}_{x}$ ferromagnetic films" Phys. Rev. Lett. 71, 2331 (1993) 
[14] A. Asamitsu, Y. Tomioka, H. Kuwahara und Y. Tokura, „Current switching of resistive states in magnetoresistive manganite", Nature 388, 50 (1997)

[15] M. Fiebig, K. Miyano, Y. Tomioka, und Y. Tokura, „Visualization of the local insulator-metal transition in $\mathrm{Pr}_{0.7} \mathrm{Ca}_{0.3} \mathrm{MnO}_{3}$ “, Science 280, 1925 (1998)

[16] E. Dagotto, „Nanoscale phase separation and colossal magnetoresistance“, Springer Verlag, Berlin (2002)

[17] E. Dagotto, T. Hotta und A. Moreo, „Colossal magnetoresistant materials: the key role of phase separation“, Phys. Rep. 344, 1 (2001)

[18] T. Becker, C. Streng, Y. Luo, V. Moshnyaga, B. Damaschke, N. Shannon und K. Samwer, „Intrinsic inhomogeneities in manganite thin films investigated with scanning tunneling spectroscopy“, Phys. Rev. Lett. 89, 237203 (2002)

[19] M. Fäth, S. Freisem, A.A. Menovsky, Y. Tomioka, J. Aarts und J. A. Mydosh, „Spatially Inhomogeneous Metal-Insulator Transition in Doped Manganites“, Science 285, 1540 (1999)

[20] M. Uehara, S. Mori, C.H. Chen und S.-W. Cheong, „Percolative phase separation underlies colossal magnetoresistance in mixed-valentmanganites", Nature 399, 560 (1999)

[21] Ch. Renner, G. Aeppli, B.-G. Kim, Yeong-Ah Soh und S.-W. Cheong, „Atomicscale images of charge ordering in a mixed-valence manganite", Nature 416, 518 (2002)

[22] Ch. Jooss, L. Wu, R.F. Klie, M. Beleggia, M. Schofield, S. Schramm, J. Hoffmann und Y. Zhu, „Polaron melting and ordering as key mechanisms for colossal resistance effects in manganites“, PNAS 104, 13597 (2007)

[23] L. Sudheendra, V. Moshnyaga, E. D. Mishina, B. Damaschke, Th. Rasing und K. Samwer, „Direct imaging of lattice-strain-induced stripe phases in an optimally doped manganite film“, Phys. Rev. B 75, 172407 (2007)

[24] L. Sudheendra, V. Moshnyaga und K. Samwer, „Metal-insulator transition and colossal magnetoresistance: Relevance of electron-lattice coupling and electronic phase separation", Contemp. Phys., eingeladener Artikel, einzureichen (2007)

[25] S.Q. Liu, N.J. Wu und A. Ignatiev, „Electric pulse-induced reversible resistance change effect in magnetoresistive films", Appl. Phys. Lett. 76, 2749 (2000)

[26] A. Ignatiev, N.J. Wu, X. Chen, S.Q. Liu, C. Papagianni und J. Strozier, „Resistance switching in perovskite thin films“, phys. stat. sol. (b) 243, 2089 (2006)

[27] H.Y. Hwang, S-W. Cheong, N.P. Ong und B. Batlogg, ,Spin-Polarized Intergrain Tunneling in $\mathrm{La}_{2 / 3} \mathrm{Sr}_{2 / 3} \mathrm{MnO}_{3}$ “, Phys. Rev. Lett. 77, 2041 (1996)

[28] R. Gross, L. Alff, B. Büchner, B.H. Freitag, C. Höfener, J. Klein, Y. Lu, W. Mader, J.B. Philipp, M.S.R. Rao, P. Reutler, S. Ritter, S. Thienhaus, S. Uhlenbruck und B. Wiedenhorst, „Physics of grain boundaries in the colossal magnetoresistance manganites", J. Magn. Magn. Mater. 211, 150 (2000) 
[29] S.A. Köster, V. Moshnyaga, K. Samwer, O. I. Lebedev, G. van Tendeloo, O. Shapoval and A. Belenchuk, „Doping of interfaces in $\left(\mathrm{La}_{0,7} \mathrm{Sr}_{0,3} \mathrm{MnO}_{3}\right)_{1-x}:(\mathrm{MgO})_{x}$ composite films" Appl. Phys. Lett. 81, 1648 (2002)

[30] Ll. Balcells, A. E. Carrillo, B. Martinez und J. Fontcuberta, „Enhanced field sensitivity close to percolation in magnetoresistive $\mathrm{La}_{2 / 3} \mathrm{Sr}_{1 / 3} \mathrm{MnO}_{3} / \mathrm{CeO}_{2}$ composites", Appl. Phys. Lett. 74, 4014 (1999)

[31] M. Ziese, „Extrinsic magnetotransport phenomena in ferromagnetic oxides“, Rep. Prog. Phys. 65, 143 (2002)

[32] G.H. Jonker und J.H. van Santen, „Ferromagnetic compounds of manganese with perovskite structure“ Physica XVI, 599 (1950)

[33] E. Wollan und W. Koehler, „Neutron Diffraction Study of the Magnetic Properties of the Series of Perovskite-Type Compounds [(1-x)La, xCa] $\mathrm{MnO}_{3}$ “ Phys. Rev. 100, 545 (1955)

[34] C. Zener, „Interaction between the $d$-shells in the transition metals. II. Ferromagnetic compounds of mangenese with perovskite structure“, Phys. Rev. 82, 403 (1951)

[35] P.W. Anderson und H. Hasegawa, „Considerations on Double Exchange“, Phys. Rev. 100, 675 (1955)

[36] P.-G. de Gennes, „Effects of Double Exchange in Magnetic Crystals“, Phys. Rev. 118, $141(1960)$

[37] S. Yunoki, A. Moreo und E. Dagotto, „Phase Separation Induced by Orbital Degrees of Freedom in Models for Manganites with Jahn-Teller Phonons“, Phys. Rev. Lett. 81, 5612 (1998)

[38] M. Mayr, A. Moreo, J.A. Vergés, J. Arispe, A. Feiguin und E. Dagotto, „Resistivity of Mixed-Phase Manganites", Phys. Rev. Lett. 86, 135 (2001)

[39] J. Burgy, M. Mayr, V. Martin-Mayor, A. Moreo und E. Dagotto, „Colossal Effects in Transition Metal Oxides Caused by Intrinsic Inhomogeneities“, Phys. Rev. Lett. 87, $277202(2001)$

[40] J.M.D. Coey, M. Viret und S. v. Molnár „Mixed valence manganites“, Adv. Phys. 48, 167 (1999)

[41] V. Moshnyaga und K. Samwer, „Ferromagnetic manganite films“, In H. Kronmüller und S.S.P. Parkin, editors, Handbook of magnetism and advanced magnetic materials, Volume 5: Spintronics and magnetoelectronics, John Wiley \& sons (2007)

[42] A. Kleine, Y. Luo und K.Samwer, „Electronic phase separation in $\mathrm{LaMnO}_{3+\delta}$-layers: Usable as a tunneling barrier?“, Europhys. Lett. 76, 135 (2006)

[43] A. Kleine, „Herstellung und Eigenschaften dünner $\mathrm{LaMnO}_{3}$-Schichten und ihre Charakterisierung als Tunnelbarriere“, Diplomarbeit, Universität Göttingen (2005) 
[44] R. Gross und A. Marx, „Spinelectronics“, Vorlesungsskript zur Vorlesung im SS 2004 an der TU München, erhältlich unter http://www.wmi.badw.de/teaching/Lecturenotes/index.html; letzter Zugriff: 05.09.2007

[45] S. Blundell, „Magnetism in condensed matter“, Oxford University Press, 2003

[46] Ch. Kittel, „Einführung in die Festkörperphysik“, 12. Auflage, Oldenbourg, 1999

[47] J.B. Goodenough, ,Theory of the role of covalence in the perovskite-type manganites [La,M(II)] $\mathrm{MnO}_{3}{ }^{*}$, Phys. Rev. 100, 564 (1955)

[48] J.B. Goodenough, „Magnetism and chemical bonds“, Interscience Publishers, New York (1963)

[49] D.I. Khomskii und G.A. Sawatzky, „Interplay between spin, charge and orbital degrees of freedom in magnetic oxides“, Sol. State Comm. 102, 87 (1997)

[50] M. Opel, „Magnetismus“, Vorlesungsskript nach der Vorlesung Magnetismus (PH-E23-6) im WS 2004/2005 an der TU München, erhältlich unter http://www.wmi.badw.de/teaching/Lecturenotes/index.html; letzter Zugriff: 05.09.2007

[51] W. Westhäuser, S. Schramm, J. Hoffmann und Ch. Jooss, „Comparative study of magnetic and electric field induced insulator-metal-transitions in $\operatorname{Pr}_{1-x} \mathrm{Ca}_{x} \mathrm{MnO}_{3}$ films", Eu. Phy. J. B 53, 323 (2006)

[52] E. Dagotto, „Open questions in CMR manganites, relevance of clustered states and analogies with other compounds including the cuprates", New Journal of Physics $7,67(2005)$

[53] G.-M. Zhao, K. Conder, H. Keller, K.H. Müller, „, Giant oxygen isotope shift in the magnetoresistive perovskite $\mathrm{La}_{1-x} \mathrm{Ca}_{x} \mathrm{MnO}_{3+y}{ }^{“}$, Nature 381, 676 (1996)

[54] A. Moreo, S. Yunoki und E. Dagotto, „Phase Separation Scenario for Manganese Oxides and Related Materials", Science 283, 2034 (1999)

[55] N. Mathur und P. Littlewood, „Mesoscopic texture in manganites“, Physics today 56, $25(2003)$

[56] V. Moshnyaga, L. Sudheendra, O. I. Lebedev, S. A. Köster, K. Gehrke, O. Shapoval, A. Belenchuk, B. Damaschke, G. van Tendeloo und K. Samwer, „A-Site Ordering versus Electronic Inhomogeneity in Colossally Magnetoresistive Manganite Films“, Phys. Rev. Lett. 97, 107205 (2006)

[57] R. Mathieu, D. Akahoshi, A. Asamitsu, Y. Tomioka und Y. Tokura, „Colossal Magnetoresistance without Phase Separation: Disorder-Induced Spin Glass State and Nanometer Scale Orbital-Charge Correlation in Half Doped Manganites“, Phys. Rev. Lett. 93, 227202 (2004)

[58] E. Gommert, H. Cerva, J. Wecker und K. Samwer, „Influence of misfit stress on the magnetoresistive properties of $\mathrm{La}_{0.7} \mathrm{Ca}_{0.3} \mathrm{MnO}_{3-\delta}$ thin films", J. Appl. Phys. 85, 5417 (1999) 
[59] A.-M. Haghiri-Gosnet und J.-P. Renard, „CMR-manganites: physics, thin films and devices", J. Phys. D: Appl. Phys. 36, R127 (2003)

[60] R.P. Borges, W. Guichard, J.G. Lunney, J.M.D. Coey und F. Ott, „Magnetic and electric „dead“ layers in $\left(\mathrm{La}_{0,7} \mathrm{Sr}_{0,3}\right) \mathrm{MnO}_{3}$ thin films“, J. Appl. Phys. 89, 3868 (2001)

[61] V. Moshnyaga, B. Damaschke, O. Shapoval, A. Belenchuk, J. Faupel, O.I. Lebedev, J. Verbeeck, G. van Tendeloo, M. Mücksch, V. Tsurkan, R. Tidecks und K. Samwer, ,Structural phase transition at the percolation threshold in epitaxial $\left(\mathrm{La}_{0,7} \mathrm{Ca}_{0,3} \mathrm{MnO}_{3}\right)_{1-x}:(\mathrm{MgO})_{x}$ nanocomposite films“, Nat. Mater. 2, 247 (2003)

[62] D.K. Petrov, L. Krusin-Elbaum, J.Z. Sun, C. Feild und P.R. Duncombe, „Enhanced magnetoresistance in sintered granular manganite/insulator systems", Appl. Phys. Lett. 75, 995 (1999)

[63] C.-H. Yan Z.-G. Xu, T. Zhu, Z.-M. Wang, F.-X. Cheng, Y.-H. Huang und C.-S. Liao, „A large low field colossal magnetoresistance in the $\mathrm{La}_{0.7} \mathrm{Sr}_{0.3} \mathrm{MnO}_{3}$ and $\mathrm{CoFe}_{2} \mathrm{O}_{4}$ combined system“, J. Appl. Phys. 87, 5588 (2000)

[64] L.E. Hueso, J. Rivas, F. Rivadulla und M.A. Lopez-Quintela, „Magnetoresistance in manganite/alumina nanocrystalline composites“, J. Appl. Phys. 89, 1746 (2001)

[65] L. Yan, L.B. Kong, T. Yang, W.C. Goh, C.Y. Tan, C.K. Ong, Md. Anisur Rahman, T. Osipowicz und M.Q. Ren, ,Enhanced low field magnetoresistance of $\mathrm{Al}_{2} \mathrm{O}_{3^{-}}$ $\mathrm{La}_{0.7} \mathrm{Sr}_{0.3} \mathrm{MnO}_{3}$ composite thin films via a pulsed laser deposition“, J. Appl. Phys. 96, 1568 (2004)

[66] S. Gupta, R. Ranjit, C. Mitra, P. Raychaudhuri und R. Pinto, „Enhanced roomtemperature magnetoresistance in $\mathrm{La}_{0.7} \mathrm{Sr}_{0.3} \mathrm{MnO}_{3}$-glass composites“, Appl. Phys. Lett. 78, 362 (2001)

[67] D. Das, P. Chowdhory, R.N. Das, C.M. Srivastava, A.K. Nigam und D. Bahadur, „Solution sol-gel processing and investigation of percolation threshold in $\mathrm{La}_{2 / 3} \mathrm{Sr}_{1 / 3} \mathrm{MnO}_{3}: x \mathrm{SiO}_{2}$ nanocomposite“, J. Magn. Magn. Mater. 238, 178 (2002)

[68] J.-M. Liu, G.L. Yuan, H.Sang, Z.C. Wu, X.Y. Chen, Z.G. Liu, Y.W. $\mathrm{Du}$, Q. Huang und C.K. Ong, „Low-field magnetoresistance in nanosized $\mathrm{La}_{0.7} \mathrm{Sr}_{0.3} \mathrm{MnO}_{3} / \mathrm{Pr}_{0.5} \mathrm{Sr}_{0.5} \mathrm{MnO}_{3}$ composites", Appl. Phys. Lett. 78, 1110 (2001)

[69] Q. Huang, J. Li, X.J. Huang, C.K. Ong und X.S. Gao, „Effect of magnetic coupling on the magnetoresistive properties in $\mathrm{La}_{0.67} \mathrm{Sr}_{0.33} \mathrm{MnO}_{3} / \mathrm{BaFe}_{11.3}(\mathrm{ZnSn})_{0.7} \mathrm{O}_{19}$ composites", J. Appl. Phys. 90, 2924 (2001)

[70] Y.-H. Huang, X. Chen, Z.-M. Wang, C.-S. Liao, C.-H. Yan, H.-W. Zhao und B.G. Shen, „Enhanced magnetoresistance in granular $\mathrm{La}_{2 / 3} \mathrm{Ca}_{1 / 3} \mathrm{MnO}_{3} /$ polymer composites“, J. Appl. Phys. 91, 7733 (2002)

[71] D. Das, A. Saha, S.E. Russek, R. Raj und D. Bahadur, „Large magnetoresistance in $\left(\mathrm{La}_{1-x} \mathrm{Ca}_{x} \mathrm{MnO}_{3}\right)_{1-y}: \mathrm{ZrO}_{2}$ composite“, J. Appl. Phys. 93, 8301 (2003) 
[72] D. Das, C.M. Srivastava, D. Bahadur, A.K. Nigam und S.K. Malik, „Magnetic and electrical transport properties of $\mathrm{La}_{0.67} \mathrm{Ca}_{0.33} \mathrm{MnO}_{3}$ (LCMO): $x \mathrm{ZnO}$ composites", J. Phys.: Condens. Matter 16, 4089 (2004)

[73] B.S. Kang, H. Wang, J.L. MacManus-Driscoll, Y. Li, Q. X. Jia, I. Mihut und J.B. Betts, „Low field magnetotransport properties of $\left(\mathrm{La}_{0,7} \mathrm{Sr}_{0,3} \mathrm{MnO}_{3}\right)_{0.5}:(\mathrm{ZnO})_{0.5}$ nanocomposite films“, Appl. Phys. Lett. 88, 192514 (2006)

[74] P. Kameli, H. Salamati, M. Eshraghi und M.R. Mohammadizadeh, „The effect of $\mathrm{TiO}_{2}$ doping on the structure and magnetic and magnetotransport properties of $\mathrm{La}_{0.75} \mathrm{Sr}_{0.25} \mathrm{MnO}_{3}$ composite“, J. Appl. Phys. 98, 043908 (2005)

[75] J. Lee, J. Park, A. Kim, K. Char, S. Park, N. Hur und S.-W. Cheong, „Phase separation in $\mathrm{La}_{5 / 8} \mathrm{Sr}_{3 / 8} \mathrm{MnO}_{3}(30 \%)+\mathrm{LuMnO}_{3}(70 \%)$ bulk sample studied by scanning microwave microscopy“, Appl. Phys. Lett. 86, 012502 (2005)

[76] S.A. Köster, „Änderung der Magnetowiderstandseffekte in $\mathrm{La}_{0,7} \mathrm{Sr}_{0,3} \mathrm{MnO}_{3}$-Filmen durch Dotierung der Korngrenzen mit $\mathrm{MgO}^{“}$, Diplomarbeit, Universität Göttingen $(2002)$

[77] Ch. M. Stingl, „Physikalisch Eigenschaften und Mikrostruktur von dünnen $\mathrm{La}_{0,67} \mathrm{Ce}_{0,33} \mathrm{MnO}_{3}$-Schichten“, Diplomarbeit, Universität Göttingen (2006)

[78] S. Valencia, O. Castano, J. Fontcuberta, B. Martynez und Ll. Balcells, „Enhanced low field magnetoresistive response in $\left(\mathrm{La}_{2 / 3} \mathrm{Sr}_{1 / 3} \mathrm{MnO}_{3}\right)_{x} /\left(\mathrm{CeO}_{2}\right)_{1-x}$ composite thick films prepared by screen printing“", J. Appl. Phys. 94, 2524 (2003)

[79] R.C. Whited, C.J. Flaten und W.C. Walker, „Exciton thermoreflectance of MgO and CaO“, Sol. State Comm. 13, 1903 (1973)

[80] C. Mitra, P. Raychaudhuri, G. Köbernik, K. Dörr, K.-H. Müller, L. Schultz und R. Pinto, , ,p-n-diode with hole- and electron-doped lanthanum manganites", Appl. Phys. Lett. 79, 2408 (2001)

[81] S. Tsunekawa, J.-T. Wang, Y. Kawazoe und A. Kasuya , „Blueshifts in the ultraviolet absorption spectra of cerium oxide nanocrystallites“, J. Appl. Phys. 94, 3654 (2003)

[82] S.K. Singh, S.B. Palmer, D.M. Paul und M. R. Lees, „Growth, transport, and magnetic properties of $\operatorname{Pr}_{0.67} \mathrm{Ca}_{0.33} \mathrm{MnO}_{3}$ thin films“, Appl. Phys. Lett. 69, 263 (1996)

[83] Y. Watanabe, J.G. Bednorz, A. Bietsch, Ch. Gerber, D. Widmer, A. Beck und S.J. Wind, „Current-driven insulator-conductor transition and nonvolatile memory in chromium-doped $\mathrm{SrTiO}_{3}$ single crystals", Appl. Phys. Lett. 78, 3738 (2001)

[84] A. Beck, J.G. Bednorz, Ch. Gerber, C. Rossel und D. Widmer, „Reproducible switching effect in thin oxide films for memory applications“, Appl. Phys. Lett. 77, $139(2000)$

[85] S. Tsui, A. Baikalov, J. Cmaidalka, Y.Y. Sun, Y.Q. Wang, Y.Y. Xue, C.W. Chu, L. Chen und A. J. Jacobson, „Field-induced resistive switching in metal-oxide interfaces“, Appl. Phys. Lett. 85, 317 (2004) 
[86] N.A. Tulina, S.A. Zver'kov, A. Arsenov, Y.M. Mukovskii und D.A. Shulyatev, „Reproducible switching in normal metal.manganite single crystal point contacts with memory effect", Physica $C$ 385, 563 (2003)

[87] Y.W. Xie, J.R. Sun, D.J. Wang, S. Liang und B.G. Shen, „Reversible electroresistance at the $\mathrm{Ag} / \mathrm{La}_{0.67} \mathrm{Sr}_{0.33} \mathrm{MnO}_{3}$ interface“, J. Appl. Phys. 100, 033704 (2006)

[88] Y.B. Nian, J. Strozier, N.J. Wu, X. Chen und A. Ignatiev, „Evidence for an oxygen diffusion model for the electric pulse induced resistance change effect in transition-metal oxides“, Phys. Rev. Lett. 98, 146403 (2007)

[89] M. Quintero, P. Levy, A.G. Leyva und M.J. Rozenberg, „Mechanism of electricpulse-induced resistance switching in manganites“, Phys. Rev. Lett. 98, 116601 (2007)

[90] G.I. Meijer, U.Staub, M. Janousch, S.L. Johnson, B.Delley und T. Neisius, „Valence states of $\mathrm{Cr}$ and the insulator-to-metal transition in Cr-doped $\mathrm{SrTiO}_{3}{ }^{*}$, Phys. Rev. B 72, 155102 (2005)

[91] R. Fors, S.I. Khartsev und A.M. Grishin, „Giant resistance switching in metalinsulator-manganite junctions: Evidence for Mott transition", Phys. Rev. B 71, 045305 (2005)

[92] M.J. Rozenberg, I.H. Inoue und M.J. Sánchez, „Nonvolatile memory with multilevel switching: a basic model“", Phys. Rev. Lett. 92, 178302 (2004)

[93] M.J. Rozenberg, I.H. Inoue und M.J. Sánchez, „Strong electron correlation effects in nonvolatile electronic memory devices“, Appl. Phys. Lett. 88, 033510 (2006)

[94] K. Szot, W. Speier, G. Bihlmayer und R. Waser, ,Switching the electric resistance of individual dislocations in single-crystalline $\mathrm{SrTiO}_{3}{ }^{*}$, Nater. Mat. 5, 312 (2006)

[95] G. Binasch, P. Grünberg, F. Saurenbach und W. Zinn, „Enhanced magnetoresistance in layered magnetic structures with antiferromagnetic interlayer exchange", Phys. Rev. B 39, 4828 (1989)

[96] M.N. Baibich, J.M. Broto, A. Fert, F. Nguyen Van Dau, F. Pertoff, P. Eitenne, G. Creuzet, A. Friederich, J. Chazelas, „Magnetoresistance of (001)Fe/(001)Cr Magnetic Superlattices“, Phys. Rev. Lett. 61, 2472 (1988)

[97] E.L. Wolf, „Principles of electron tunneling spectroscopy“, Oxford University Press, 1985

[98] J.G. Simmons, „Generalized formula for the electric tunnel effect between similar electrodes separated by a thin insulating film“, J. Appl. Phys. 34, 1793 (1963)

[99] J.J. Åkerman, R. Escudero, C. Leighton, S. Kim, D.A. Rabson, R.W. Dave, J.M. Slaughter und I K. Schuller, „Criteria for ferromagnetic-insulator-ferromagnetic tunneling“, J. Magn. Magn. Mater. 240, 86 (2002)

[100] W.F. Brinkman, R.C. Dynes und J.M. Rowell , „Tunneling Conductance of Asymmetrical Barriers“, J. Appl. Phys. 41, 1915 (1970) 
[101] N.F. Mott, „The Electrical Conductivity of Transition Metals“, Proc. Roy. Soc. 153, 699 (1936)

[102] D. Bürgler und P. Grünberg, „Magnetoelectronics: Exchange Anisotropy, Interlayer Exchange Coupling, GMR, TMR and Current-induced Magnetic Switching“, 32. IFF Ferienkurs, Schriften des Forschungszentrums Jülich, Materie und Material, Band 7 (2001)

[103] R.A. de Groot, F. M. Mueller, P.G. van Engen undK. H. J. Buschow, „New Class of Materials: Half-Metallic Ferromagnets", Phys. Rev. Lett.50, 2024 (1983)

[104] T. Ishikawa, T. Marukame, H. Kijima, K.-I. Matsuda, T. Uemura, M. Arita und M. Yamamoto, ,Spin-dependent tunneling characteristics of fully epitaxial magnetic tunneling junctions with a full-Heusler alloy $\mathrm{Co}_{2} \mathrm{MnSi}$ thin film and a $\mathrm{MgO}$ tunnel barrier" Appl. Phys. Lett. 89, 192505 (2006)

[105] Yu.S. Dedkov, U. Rüdiger und G. Güntherodt „Evidence for the half-metallic ferromagnetic state of $\mathrm{Fe}_{3} \mathrm{O}_{4}$ by spin-resolved photoelectron spectroscopy“, Phys. Rev. B 65, 064417 (2002)

[106] M. Fonin, Y. Dedkov, C. König, G. Güntherodt, U. Rüdiger, J. Mayer, D. Vyalikh und S. Molodtsov, „Room Temperature Spin Polarization of Epitaxial HalfMetallic $\mathrm{Fe}_{3} \mathrm{O}_{4}$ (111) and $\mathrm{CrO}_{2}$ (100) Films“ Advanced Solid State Physics 43, Springer, Berlin (2003)

[107] E. Goering, A. Bayer, S. Gold, G. Schütz, M. Rabe, U. Rüdiger und G. Güntherodt, „Strong Anisotropy of Projected 3d Moments in Epitaxial $\mathrm{CrO}_{2}$ Films“, Phys. Rev. Lett. 88, 207203 (2002)

[108] Yu.S. Dedkov, M. Fonine, C. Konig, U. Rudiger, G. Guntherodt, S. Senz und D. Hesse, „Room-temperature observation of high-spin polarization of epitaxial $\mathrm{CrO}_{2}(100)$ island films at the Fermi energy", Appl. Phys. Lett. 80, 4181 (2002)

[109] J.C. Slonczewski, „Conductance and exchange coupling of two ferromagnets separated by a tunneling barrier“, Phys. Rev. B 39, 6995 (1989)

[110] A.M. Bratkovsky, ,"Tunneling of electrons in conventional and half-metallic systems: Towards very large magnetoresistance“, Phys. Rev. B 56, 2344 (1997)

[111] W.H. Butler, X.-G. Zhang, T.C. Schulthess und J.M. MacLaren, „Spin-dependent tunneling conductance of $\mathrm{Fe} / \mathrm{MgO} / \mathrm{Fe}$ sandwiches", Phys. Rev. B 63, 054416 (2001)

[112] D. Djayaprawira, K. Tsunekawa, M. Nagai, H. Maehara, S. Yamagata, N. Watanabe, S. Yuasa, Y. Suzuki und K. Ando, „230\% room-temperature magnetoresistance in $\mathrm{CoFeB} / \mathrm{MgO} / \mathrm{CoFeB}$ magnetic tunnel junctions", Appl. Phys. Lett. 86, 092502 (2005)

[113] K. Tsunekawa, D. Djayaprawira, M. Nagai, H. Maehara, S. Yamagata, N. Watanabe, S. Yuasa, Y. Suzuki und K. Ando, „Giant tunneling magnetoresistance effect in low-resistance $\mathrm{CoFeB} / \mathrm{MgO}(001) / \mathrm{CoFeB}$ magnetic tunnel junctions for readhead applications", Appl. Phys. Lett. 87, 072503 (2005) 
[114] M. Bowen, M. Bibes, A. Barthélèmy, J.-P. Contour, A. Anane, Y. Lemaître und A. Fert, „Nearly total spin polarization in $\mathrm{La}_{2} / 3 \mathrm{Sr}_{2 / 3} \mathrm{MnO}_{3}$ from tunneling experiments", Appl. Phys. Lett. 82, 233 (2003)

[115] J.-H. Park, E. Vescovo, H.-J. Kim, C. Kwon, R. Ramesh und T. Venkatesan, „Magnetic Properties at Surface Boundary of a Half-Metallic Ferromagnet $\mathrm{La}_{0,7} \mathrm{Sr}_{0,3} \mathrm{MnO}_{3}{ }^{\text {", }}$ Phys. Rev. Lett. 81, 1953 (1998)

[116] J.S. Moodera, J. Nowak und R.J.M. van de Veerdonk, „Interface Magnetism and Spin Wave Scattering in Ferromagnet-Insulator-Ferromagnet Tunnel Junctions“, Phys. Rev. Lett. 80, 2941 (1998)

[117] J.S. Moodera und G. Mathon, ,Spin polarized tunneling in ferromagnetic junctions“, J. Magn. Magn. Mater. 200, 248 (1999)

[118] S. Zhang, P.M. Levy, A.C. Marley und S.S.P. Parkin, „Quenching of Magnetoresistance by Hot Electrons in Magnetic Tunnel Junctions“, Phys. Rev. Lett. 79, 3744 (1997)

[119] A.M. Bratkovsky, „Assisted tunneling in ferromagnetic junctions and half-metallic oxides“, Appl. Phys. Lett. 72, 2334 (1998)

[120] T. Dimopoulos, Y. Henry, V. Da Costa, C. Tiusan und K. Ounadjela, „Influence of barrier overoxidation and annealing on the inelastic spin-dependent tunneling in AlO ${ }_{x}$-based junctions“, J. Appl. Phys. 95, 6936 (2004)

[121] L.I. Glazman und K.A. Matveev, „Inelastic tunneling across thin amorphous films“, Sov. Phys. JEPT 67, 1276 (1988)

[122] J.A. Katine, F.J. Albert, R.A. Buhrman, E.B. Myers und D.C. Ralph, „CurrentDriven Magnetization Reversal and Spin-Wave Excitations in $\mathrm{Co} / \mathrm{Cu} / \mathrm{Co}$ Pillars“, Phys. Rev. Lett. 84, 3149 (2000)

[123] D. Bürgler, „Advanced magnetic switching concepts“, 36. IFF Ferienkurs, Schriften des Forschungszentrums Jülich, Materie und Material, Band 26 (2001)

[124] M.D. Stiles und A. Zangwill, „Anatomy of spin-transfer torque“, Phys. Rev B 66, 014407 (2002)

[125] F.J. Albert, N.C. Emley, E.B. Myers, D.C. Ralph und R. A. Buhrman „Quantitative Study of Magnetization Reversal by Spin-Polarized Current in Magnetic Multilayer Nanopillars“, Phys. Rev. Lett. 88, 226802 (2002)

[126] J.Z. Sun, „Current-driven magnetic switching in manganite trilayer junctions“, $J$. Magn. Magn. Mater. 202, 157 (1999)

[127] M. Bibes, Ll. Balcells, S. Valencia, J. Fontcuberta, M. Wojcik, E. Jedryka und S. Nadolski, „Nanoscale Multiphase Separation at $\mathrm{La}_{2 / 3} \mathrm{Ca}_{1 / 3} \mathrm{MnO}_{3} / \mathrm{SrTiO}_{3}$ Interfaces“, Phys. Rev. Lett. 87, 067210 (2001)

[128] M. Bibes, Ll. Balcells, J. Fontcuberta, M. Wojcik, S. Nadolski und E. Jedryka, ,Surface-induced phase separation in manganites: A microscopic origin for powder magnetoresistance“, Appl. Phys. Lett. 82, 928 (2003) 
[129] N.D. Mathur, G. Burnell, S.P. Isaac, T.J. Jackson, B.-S. Teo, J.L. MacManusDriscoll, L.F. Cohen, J.E. Evetts und M.G. Blamire, „, Large low-field magnetoresistance in $\mathrm{La}_{0.7} \mathrm{Ca}_{0.3} \mathrm{MnO}_{3}$ induced by artificial grain boundaries", Nature 387, 266 (1997)

[130] K. Steenbeck, T. Eick, K. Kirsch, H.-G. Schmidt und E. Steinbeiss, „Tunneling-like magnetoresistance in bicrystal $\mathrm{La}_{0.8} \mathrm{Sr}_{0.2} \mathrm{MnO}_{3-\delta}$ thin films", Appl. Phys. Lett. 73, 2506 (1998)

[131] N.K. Todd, N.D. Mathur, S.P. Isaac, J.E. Evetts und M. G. Blamire, „Currentvoltage characteristics and electrical transport properties of grain boundaries in $\mathrm{La}_{1-x}(\mathrm{Sr} / \mathrm{Ca})_{x} \mathrm{MnO}_{3}$ “, J. Appl. Phys. 85, 7263 (1999)

[132] W. Westerburg, F. Martins, S. Friedrich, M. Maier und G. Jakob, „Current dependence of grain boundary magnetoresistance in $\mathrm{La}_{0.66} \mathrm{Ca}_{0.33} \mathrm{MnO}_{3}$ films", J. Appl. Phys. 86, 2173 (1999)

[133] Y.-A. Soh, G. Aeppli, N.D. Mathur und M.G. Blamire, „Mesoscale magnetism at the grain-boundaries in colossal magnetoresistive films", Phys. Rev. B 63, 020402 (2000)

[134] R. Mathieu, P. Svedlindh, R. Gunnarsson und Z.G. Ivanov, „Magnetic contribution to the resistivity noise in a $\mathrm{La}_{0.7} \mathrm{Sr}_{0.3} \mathrm{MnO}_{3}$ film grain boundary", Phys. Rev B 63, 132407 (2001)

[135] R. Gunnarsson, Z.G. Ivanov, C. Dubourdieu und H. Roussel, „, Low-field magnetoresistance in perovskite manganites: Magnetic field, temperature, and current dependence“, Phys. Rev. B 69, 054413 (2004)

[136] J.B. Philipp, L. Alff, A. Marx und R. Gross, „Low-frequency $1 / f$ noise in doped manganite grain-boundary junctions“, Phys. Rev. B 66, 224417 (2002)

[137] J.E. Evetts, M.G. Blamire, N.D. Mathur, S.P. Isac, B.-S. Teo, L.F. Cohen und J.L. MacManus-Driscoll, „Defect-induced spin disorder and magnetoresistance in single-crystal and polycrystalline rare-earth manganites thin films", Phil. Trans. R. Soc. Lond. A 356, 1593 (1998)

[138] F. Guinea, ,Spin-flip scattering in magnetic junctions“, Phys. Rev B 58, 9212 (1998)

[139] M. Ziese, „Grain-boundary magnetoresistance in manganites: Spin-polarized inelastic tunneling through a spin-glass-like barrier", Phys. Rev. B 60, 738 (1999)

[140] J. Klein, C. Höfener, S. Uhlenbruck, L. Alff, B. Büchener und R. Gross, „On the nature of grain boundaries in the colossal magnetoresistance manganites“, Europhys. Lett. 47, 371 (1999)

[141] H. Yamada, Y. Ogawa, Y. Ishii, H. Sato, M. Kawasaki, H. Akoh und Y. Tokura, „Engineered Interface of Magnetic Oxides“, Science 305, 646 (2004)

[142] A.K. Raychaudhuri, „Measurement of $1 / f$ noise nd its application in material science“, Curr. Opin. Solid State Mater. Sci. 6, 67 (2002) 
[143] P. Dutta und P.M. Horn, „Low-frequency fluctuations in solids: $1 / f$ noise“, Rev. Mod. Phys. 53, 497 (1981)

[144] M.B. Weissman , $1 / f$ noise and other slow, nonexponential kinetics in condensed matter", Rev. Mod. Phys. 60, 537 (1988)

[145] Sh. Kogan, „Electronic noise and fluctuations in solids“, Cambridge University Press, 1996

[146] N. Wiener, „Generalized Harmonic Analysis“ Acta Mathematica 55, 117 (1930)

[147] A. Khintchine, „Korrelationstheorie der stationären stochastischen Prozesse“, Math. Ann. 109, 604 (1934)

[148] W. Schottky, „Über spontane Stromschwankungen in verschiedenen Elektrizitätsleitern“, Ann. Phys. 362, 541 (1918)

[149] J.B. Johnson, „The Schottky Effect in Low Frequency Circuits“, Phys. Rev. 26, 71 (1925)

[150] W. Schottky, „Small-Shot Effect and Flicker Effect „Phys. Rev. 28, 74 (1926)

[151] R.F. Voss und J. Clark, „Flicker (1/f) noise: Equilibrium temperature and resistance fluctuations" Phys. Rev. B 13, 556 (1976)

[152] F.N. Hooge und A.M.H. Hoppenbrouwers, „Amplitude distribution of $1 / f$ noise“ Physica 42, 331 (1969)

[153] S.K. Arora, R. Kumar, R. Singh, D. Kanjilal, G.K. Mehta, R. Bathe, S.I. Patil und S. B. Ogale „Electronic transport and $1 /$ f noise studies in $250 \mathrm{MeV}{ }^{107} \mathrm{Ag}$ ion irradiated $\mathrm{La}_{0.75} \mathrm{Ca}_{0.25} \mathrm{MnO}_{3}$ thin films"

[154] P. Reutler, A. Bensaid, F. Herbstritt, C. Höfener, A. Marx und R. Gross, „Local magnetic order in manganite thin films studied by $1 / f$ noise measurements", Phys. Rev. B 62, 11619 (2000)

[155] B. Raquet, A. Anane, S. Wirth, P. Xiong und S. von Molnár „Noise Probe of the Dynamic Phase Separation in $\mathrm{La}_{2 / 3} \mathrm{Ca}_{1 / 3} \mathrm{MnO}_{3}$ “, Phys. Rev. Lett. 84, 4485 (2002)

[156] V. Podzorov, M. Uehara, M.E. Gershenson, T.Y. Koo und S-W. Cheong, „Giant 1/f noise in perovskite manganites: Evidence of the percolation threshold“, Phys. Rev. B 61, R3784 (2000)

[157] H.T. Hardner, M.B. Weissman, M. Jaime, R.E. Treece, P.C. Dorsey, J.S. Horwitz und D.B. Chrisey, „Non-Gaussian noise in a colossal magnetoresistive film“, $J$. Appl. Phys. 81, 272 (1997)

[158] R.D. Merithew, M.B. Weissman, F.M. Hess, P. Spradling, E.R. Nowak, J. O’Donnell, J.N. Eckstein, Y. Tokura und Y. Tomioka, „Mesoscopic Thermodynamics of an Inhomogeneous Colossal-Magnetoresistive Phase", Phys. Rev. Lett. 84, 3442 (2000)

[159] V. Podzorov, M.E. Gershenson, M. Uehara und S-W. Cheong, „Phase separation and $1 / f$ noise in low- $T_{M I}$ colossal magnetoresistance manganites", Phys. Rev. B 64, $115113(2001)$ 
[160] F.M. Hess, R.D. Merithew, M.B. Weissman, Y. Tokura und Y. Tomioka, „Mesoscopic thermodynamics of an inhomogeneous colossal magnetoresistive phase in a bulk crystal", Phys. Rev. B 63, 180408 (2001)

[161] A. Palanisami, M.B. Weissman und N.D. Mathur, „Magnetoresistive dynamics and noise in low-strain manganite films“, Phys. Rev. B 71, 014423 (2005)

[162] X. Liu und G. Xiao, ,Thermal annealing effects on low-frequency noise and transfer behavior in magnetic tunnel junction sensors“, J. Appl. Phys. 94, 6218 (2003)

[163] H.J. Shim, I.J. Hwang, K.S. Kim und B.K. Cho, J.-T. Kim und J.H. Sok, „Voltage dependence of magnetoresistance in magnetic tunnel junctions with AlN tunnel barrier", J. Appl. Phys. 92, 1095 (2002)

[164] E.R. Nowak, R.D. Merithew, M.B. Weissman, I. Bloom und S.S.P. Parkin, „Noise properties of ferromagnetic tunnel junctions“, J. Appl. Phys. 84, 6195 (1998)

[165] E.R. Nowak, M.B. Weissman und S.S.P. Parkin, „Electric noise in hysteretic ferromagnet-insulator-ferromagnet tunnel junctions“, Appl. Phys. Lett. 74, 600 (1999)

[166] S. Ingvarsson, G. Xiao, S.S.P. Parkin, W.J. Gallagher, G. Grinstein und R.H. Koch, „Low-Frequency Magnetic Noise in Micron-Scale Magnetic Tunnel Junctions“, Phys. Rev. Lett. 85, 3289 (2000)

[167] S. Ingvarsson, G. Xiao, R.A. Wanner, P. Trouilloud, Y. Lu, W.J. Gallagher, A. Marley, K.P. Roche und S.S.P. Parkin, „Electronic noise in magnetic tunnel junctions", J. Appl. Phys. 85, 5270 (1999)

[168] K.S. Kim, H.J. Shim, I.J. Hwang, B.K. Cho, J.H. Seok und J.-T. Kim, „Magnetic field dependent noise in magnetic tunnel junction“, J. Appl. Phys. 91, 8804 (2002)

[169] V. Moshnyaga, I. Khoroshun, A. Sidorenko, P. Petrenko, A. Weidinger, M. Zeitler, B. Rauschenbach, R. Tidecks und K. Samwer, "Preparation of rare-earth manganite-oxide thin films by metalorganic aerosol deposition technique“, Appl. Phys. Lett. 74, 2842 (1999)

[170] I.V. Khoroshun, E.V. Karyaev, V.T. Moshnyaga, G.A. Kiosse, M.A. Krachum, V.M. Zakosarenko und V.Yu. Davydov, „Characteristics of epitaxial Y-Ba-Cu-O thin films grown by aerosol MOCVD technique“, Supercond. Sci. Technol. 3, 493 (1990)

[171] A.I. Ivashchenko, E.V. Karyaev, I.V. Khoroshun, G.A. Kiosse, V.T. Moshnyaga und P.A. Petrenko, „Relations between structural and electronic properties of $\mathrm{SnO}_{2}$ polycrystalline thin films prepared by the aerosol MOCVD technique“, Thin Solid Films 263, 122 (1995)

[172] L. Kulyuk, I. Kravetsky, A. Micu, E.V. Karyaev, V.T. Moshnyaga, I.V. Khoroshun, I. Muscutariu und V. Sokolyuk, „Nonlinear Optics of Low-Dimensional Structures and New Materials“, Proc. SPIE 2801, 135 (1995) 


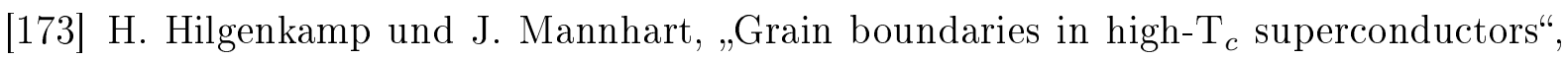
Rev. Mod. Phys. 74, 485 (2002)

[174] G. Gottstein, Physikalische Gundlagen der Materialkunde, Springer Verlag, Berlin Heidelberg (2001)

[175] B.D. Cullity, Elements Of X-Ray Diffraction, Addison-Wesley, Reading (MA) (1978)

[176] O. Seeck, „Analytic Methods“, In 32. IFF Ferienkurs, Neue Materialien für die Informationstechnik, Jülich, 2001

[177] A. Segmüller, „Observation of x-ray interferences on thin films of amorphous silicon“, Thin solid films 18, 287 (1973)

[178] W. Hink und W. Petzold, „X-ray interference patterns of vapor deposited aluminum layers“, Z. Angew. Physik 10, 553 (1958)

[179] A. Guinier, x-ray diffraction in crystals, imperfect crystals and amorphous bodies, Dover publications, Inc., New York (1994)

[180] 27. IFF Ferienkurs, Streumethoden zur Untersuchung kondensierter Materie, Jülich, 1996

[181] W. Buckel und R. Kleiner Supraleitung, Wilex-VCH, Weinheim (2004)

[182] Carl Zeiss SMT AG, private Mitteilung

[183] B. Fultz und J.M. Howe, ,Transmission Electron Microscopy and Diffractometry of Materials“, 2. Auflage, Springer Verlag, Berlin (2002)

[184] http://www.qdusa.com/pdf/brochures/ppms_9_06.pdf; letzter Zugriff: 05.09.2007

[185] Oxford Research Instuments, UK, Manual for MagLab System 2000 (1999)

[186] G. Meis, Entwicklung, Fertigung und Test eines Probenhalters für Arbeitstemperaturen zwischen 4,2 K und 130 K, Diplomarbeit, Fachhochschule Heilbronn (1993)

[187] A. Marx, Niederfrequentes 1/f-Rauschen in Josephson-Kontakten aus Hochtemperatur-Supraleitern, Shaker-Verlag, Aachen (1996)

[188] F.N. Herbstritt, Ladungstransport und Rauschen in submikrometer-strukturierten Korngrenzenkontakten aus $\mathrm{YBa}_{2} \mathrm{Cu}_{3} \mathrm{O}_{7-\delta}$, Dissertation, Universität Köln (2001)

[189] Ll. Balcells, J. Fontcuberta, B. Martinez und X. Obradors, „High-field magnetoresistance at interfaces in manganese perovskites“, Phys. Rev. B 58, 14697 (1998)

[190] J.S. Helman und B. Abeles, ,Tunneling of spin-polarized electrons and magnetoresistance in granular Ni films", Phys. Rev. Lett. 37, 1429 (1976)

[191] A.L. Efros und B.I. Shklovskii, „Coulomb gap and low temperature conductivity of disordered systems", J. Phys. C: Solid State Phys. 8 (1975)

[192] L.D. Yao, W. Zhang, J.S. Zhang, H. Yang, F.Y. Li, Z.X. Liu, C.Q. Jin und R.C. $\mathrm{Yu}$, ,Enhanced magnetoresistance of $\mathrm{La}_{2 / 3} \mathrm{Ca}_{1 / 3} \mathrm{MnO}_{3}$ nanocrystalline composites synthesized by polymer-network gel method“, J. Appl. Phys. 101, 063905 (2007) 
[193] K.M. Ryan, J.P. McGrath, R.A. Farell, W.M. O’Neill, C.J. Barnes und M.A. Morris, „Measurements of the lattice constants of ceria when doped with lanthana and praseodymia“, J. Phys.: Condens. Matter 15, L49 (2003)

[194] S. Kirkpatrick, „Percolation and Conduction“, Rev. Mod. Phys. 45, 574 (1973)

[195] D. Craik, Magnetism: principles and applications, Wiley, Chichester (1995)

[196] R.C. O'Handley, Modern magnetic materials: principles and applications, Wiley, New York (2000)

[197] J. Wu, H. Sun und Z.Y. Li, „Grain-boundary magnetoresistance enhancement induced by network self-optimization“, J. Appl. Phys. 99, 053907 (2006)

[198] T. Dimopoulos, G. Gieres, J. Wecker, Y. Luo und K. Samwer, „Analysis of the magnetotransport channels in tunnel junctions with amorphous CoFeB“, Europhys. Lett. 68, 706 (2004)

[199] J.A. Appelbaum, „Exchange Model of Zero-Bias Tunneling Anomalies“, Phys. Rev. 154, 633 (1967)

[200] A. Baikalov, Y.Q. Wang, B. Shen, B. Lorenz, S. Tsui, Y.Y. Sun, Y.Y. Xue und C.W. Chu, „Field-driven hysteretic and reversible resistive switch at the Ag$\mathrm{Pr}_{0.7} \mathrm{Ca}_{0.3} \mathrm{MnO}_{3}$ interface“, Appl. Phys. Lett. 83, 957 (2003)

[201] Y. Jiang, S. Wang, Y. Zhang, J. Yan und W. Li, „Kinetic study of the formation of oxygen vacancy on lanthanum manganite electrodes", J. Electrochem. Soc. 145, 373 (1998)

[202] X. Chen, N. J. Wu, J. Strozier und A. Ignatiev, „Direct resistance profile for an electrical pulse induced resistance change device“, Appl. Phys. Lett. 87, 233506 (2005)

[203] X. Chen, J. Strozier, N.J. Wu, A. Ignatiev und Y.B. Nian, „A study of symmetry properties and multi-state nature of perovskite oxide-based electrical pulse induced resistance-change devices", New J. Phys. 8, 229 (2006)

[204] Yeong-Ah Soh, P. G. Evans, Z. Cai, B. Lai, C.-Y. Kim, G. Aeppli, N. D. Mathur, M. G. Blamire, and E. D. Isaacs, „Local mapping of strain at grain boundaries in colossal magnetoresistive films using x-ray microdiffraction", J. Appl. Phys. 91, 7742 (2002)

[205] F. Giesen, B. Damaschke, V. Moshnyaga, K. Samwer und G.A. Müller, „Suppression of interface-induced electronic phase separation in all-manganite multilayers by preservation of the Mn-O chain network“, Phys. Rev. B 69, 014421 (2004)

[206] J.F. Scott, „Multiferroic memories“, Nature Mater. 6, 256 (2007)

[207] M. Gajek, M. Bibes, S. Fusil, K. Bouzehouane, J. Fontcuberta, A. Barthélémy, A. Fert, „Tunnel junctions with multiferrois barriers“, Nature Mater. 6, 296 (2007)

[208] M.G. Blamire, C.W. Schneider, G. Hammerl und J. Mannhart, „Conduction and magnetoresistance in doped manganite grain boundaries“, Appl. Phys. Lett. 82, $2670(2003)$ 
[209] G. Hammerl, A. Schmehl, R.R. Schulz, B. Goetz, H. Bielefeldt, C.W. Schneider, H. Hilgenkamp und J. Mannhart, ,Enhanced supercurrent density in polycrystalline $\mathrm{YBa}_{2} \mathrm{Cu}_{3} \mathrm{O}_{7-\delta}$ at $77 \mathrm{~K}$ from calcium doping of grain boundaries“, Nature 407, $162(2000)$

[210] V. Moshnyaga, B. Damaschke, O. Shapoval, A. Belechnuk, J. Faupel, O.I. Lebedev, J. Verbeek, G. van Tendeloo, M. Mücksch, V. Tsurkan, R. Tidecks und K. Samwer, ,Structural phase transition at the percolation thershold in epitaxial $\left(\mathrm{La}_{0.7} \mathrm{Ca}_{0.3} \mathrm{MnO}_{3}\right)_{1-x}:(\mathrm{MgO})_{x}$ nanocomosite films“, Nature Mater. 2, 247 (2003)

[211] R. Gunnarsson, M. Hanson und C. Dubourdieu, ,Stoner-Wohlfarth model applied to bicrystal magnetoresistance hysteresis“, J. Appl. Phys. 96, 482 (2004)

[212] R. Gunnarsson und M. Hanson, „Misorientation angle dependence of bicrystal magnetoresistance within the model of coherent rotation", J. Phys.: Condens. Matter 16, 3761 (2004)

[213] P. Lecoeur, P.L. Trouilloud, Gang Xiao, A. Gupta, G.Q. Gong und X.W. Li, „Magnetic domain structures of $\mathrm{La}_{0.67} \mathrm{Sr}_{0.33} \mathrm{MnO}_{3}$ thin films with different morphologies" J. Appl. Phys. 82, 3934 (1997)

[214] K. Steenbeck und R. Hiergeist, „Magnetic anisotropy of ferromagnetic $\operatorname{La}_{0.7}(\mathrm{Sr}$, Ca) ${ }_{0.3} \mathrm{MnO}_{3}$ epitaxial films", Appl. Phys. Lett. 75, 1778 (1999)

[215] K.-K. Choi, T. Taniyama und Y. Yamazaki, „Strain-induced anisotropic low-field magnetoresistance of La-Sr-Mn-O thin films“ J. Appl. Phys. 90, 6145 (2001)

[216] J.B. Philipp, C. Höfener, S. Thienhaus, J. Klein, L. Alff und R. Gross, „Large twolevel magnetoresistance effect in doped manganite grain-boundary junctions“, Phys. Rev. B 62, 9248 (2000)

[217] A.E. Berkowitz, K. Takano, „Exchange anisotropy - a review“, J. Magn. Magn. Mater. 200, 552 (1999)

[218] J. Nogués und I.K. Schuller, „Exchange bias“, J. Magn. Magn. Mater. 192, 203 (1999)

[219] C. Höfener, J.B. Philipp, J. Klain, L. Alff, A. Marx, B. Büchner und R. Gross, „Voltage and temperature dependence of the grain boundary tunneling magnetoresistance in manganites", Europhys. Lett. 50, 681 (2000)

[220] K.H. Fischer und J.A. Hertz, Spin Glasses, Cambridge University Press, Cambridge, 1993

[221] R. Gunnarsson, A. Kadigrobov und Z. Ivanov, „Model for spin-polarized transport in perovskite manganite bicrystal grain boundaries", Phys. Rev. B 66, 024404 (2002)

[222] C.H. Marrows, „Spin-polarised currents and magnetic domain walls“, Adv. Phys. 54, $585(2005)$

[223] A.D. Kent, J. Yu, U. Rüdiger and S.S.P. Parkin, „Domain wall resistivity in epitaxial thin film microstructures" J. Phys.: Condens. Matter 13, R461 (2001) 
[224] J. Wolfman, A.M. Haghiri-Gosnet, B. Raveau, C. Vieu, E. Cambril, A. Cornette und H. Launois, „Large domain wall magnetoresistance up to room temperature in $\mathrm{La}_{0.7} \mathrm{Sr}_{0.3} \mathrm{MnO}_{3}$ bridges with nanoconstrictions“ J. Appl. Phys. 89, 6955 (2001)

[225] I. Pallecchi, L. Pellegrino, A. Caviglia, E. Bellingeri, G. Canu, G.C. Gazzadi, A.S.Siri und D Marré, „Current-driven hystersis effects in manganite spintronic devices“, Phys. Rev. B 74, 014434 (2006)

[226] J.Y. Gu, S.B. Ogale, M. Rajeswari, T. Venkatesan, R. Ramesh, V. Radmilovic, U. Dahmen, G. Thomas und T. W. Noh, "In-plane grain boundary effects on the magnetotransport properties of $\mathrm{La}_{0.7} \mathrm{Sr}_{0.3} \mathrm{MnO}_{3-\delta}$ ", Appl. Phys. Lett. 72, 1113 (1998)

[227] Ch. Denker und V. Radisch, private Mitteilung

[228] R. Menzel, K. Gartner, W. Wesch und H. Hobert, „Damage production in semiconductor materials by a focused $\mathrm{Ga}^{+}$ion beam“, J. Appl. Phys. 88, 5658 (2000)

[229] L. Sudheendra, M. Esseling, V. Moshnyaga, B. Damaschke und K. Samwer, „Realization of a colossal effect on a manganite film by STM-tip induced electronic phase separation", unveröffentlicht (2007) 


\section{Publikationen}

\section{Artikel}

- M. Esseling, Y. Luo, and K. Samwer ,Structural and magnetic properties of oxide films CoFeHfO: a possible candidate for all-oxide TMR-junctions?“, Europhys. Lett. 68, 100 (2004).

- Y. Luo, M. Esseling, A. Käufler, K. Samwer, T. Dimopoulos, G. Gieres, M. Rührig, J. Wecker, C. Rudolf, T. Niermann, and M. Seibt „Co-rich magnetic amorphous films and their application in magnetoelectronics“, Phys. Rev. B 72, 014426 (2005).

- M. Esseling, V. Moshnyaga, K. Samwer, A. Marx, and R. Gross „Low-frequency 1/f-noise in LSMO:MgO nanocomposite films“, Appl. Phys. Lett. 87, 082509 (2005).

- Y. Luo, M. Esseling, K. Zhang, G. Güntherodt, and K. Samwer „Magnetoresistance in amorphous oxide films CoFeHfO“, Europhys. Lett. 73, 415 (2006).

- Y. Luo, M. Esseling, M. Münzenberg, and K. Samwer „A novel spin transfer torque effect in $\mathrm{Ag}_{2}$ Co granular films", New Journal of Physics, angenommen zur Veröffentlichung (2007).

\section{Beiträge auf Konferenzen}

- Frühjahrstagung der Deutschen Physikalischen Gesellschaft, Regensburg, 2002

- Frühjahrstagung der Deutschen Physikalischen Gesellschaft, Dresden, 2003

- Frühjahrstagung der Deutschen Physikalischen Gesellschaft, Berlin, 2005

- Edgar-Lüscher-Seminar, Serneus, Schweiz, 2006

- Frühjahrstagung der Deutschen Physikalischen Gesellschaft, Dresden, 2006

- Edgar-Lüscher-Seminar, Serneus, Schweiz, 2007

- 10th Joint MMM/Intermag Conference, Baltimore, 2007

- Frühjahrstagung der Deutschen Physikalischen Gesellschaft, Regensburg, 2007 



\section{Danksagung}

Im Laufe meiner Jahre am I. Physikalischen Institut haben viele Menschen zum Gelingen dieser Arbeit beigetragen. Ihnen gilt an dieser Stelle mein besonderer Dank.

Prof. Dr. K. Samwer danke ich für die Möglichkeit, meine Dissertation am I. Physikalischen Institut anfertigen zu können. Sein großes Interesse am Fortgang dieser Arbeit sowie der Freiraum der Ausgestaltung waren immer Motivation für mich. Ich danke auch für die Finanzierung zahlreicher Seminare, Konferenzen und Ferienschulen. Nicht unerwähnt lassen kann ich die durchaus kontrovers geführten, aber von fachlicher Kompetenz geprägten Fussballdiskussionen, besonders nach großartigen Nord-Süd-Gipfeln.

$P D$ Christian Jooss danke ich herzlich für die Übernahme des Korreferats und hilfreiche Diskussionen, im Besonderen die EPIR-Experimente betreffend.

PD Vasily Moshnyaga war immer kurzfristig zu langen Diskussionen bereit. Sein unerschöpfliches Wissen über Experimentiertechnik und hochkorrelierte Elektronensysteme hat diese Arbeit ein großes Stück voran gebracht. Danke für Deine Offenheit, die so viele anregende Diskussionen möglich gemacht hat.

Prof. Dr. R. Gross und Dr. Achim Marx vom WMI Garching haben mit großartigen Instrumenten, Unterbringung direkt an der Isar und Unmengen echt italienischen Kaffees einen besonderen Beitrag zu dieser Arbeit geleistet. Dank an alle Mitarbeiter des WMI, besonders Karl Madek und Georg Wild. Es war ein Spaß mit Euch im Labor.

Christian Stingl danke ich herzlich für die prompte Übernahme des Lektorats. Mit seiner Hilfe konnten manche Verständnisschwierigkeiten ausgeräumt werden.

Herrn Volker Radisch, PD Michael Seibt und Dr. Karsten Thiel danke ich für die Hilfe und die lustigen Stunden am FIB und TEM. Dr. Sebastian Dreyer (Keule) und Michael Malchow haben mich hervorragend in das Ionenstrahlätzen eingeführt, und so die Mikrostrukturierung von Manganaten überhaupt erst ermöglicht. In diesem Zusammenhang geht ein herzlicher Dank an meine Mitstreiterin bei den ersten Schritten in der Elektronenstrahl-Lithographie Dr. Anne Parge für die gemeinsame Bewältigung der diffizilen Problematiken am REM.

Hamish Gordon sei für die Überlassung seiner im Rahmen des Austauschprojektes angefertigten Proben gedankt.

Viele Diskussionen in der Manganatgruppe haben zum Verständnis der experimentellen Ergebnisse beigetragen. Dem wandelnden Manganatlexikon Dr. L. Sudheendra (Sudhee) gilt hier besonderer Dank für die Unterstützung, insbesondere bei der Interpretation der spannungsinduzierten Widerstandsänderungen. Prof. Dr. W. Felsch danke ich für das Diskutieren der mikromagnetischen Schwierigkeiten in FIB-Kontakten. 
Allen Mitarbeitern des I. Physikalischen Instituts danke ich für die herzliche Atmosphäre. Ich habe es immer als etwas Besonderes empfunden, in diesem Rahmen arbeiten zu dürfen. Grill- und Fussballabende oder auch die Blockseminare in den Bergen oder an der See werden immer erst durch die Menschen zum Erlebnis. Speziell herausheben möchte ich an dieser Stelle Uta Bete, Dr. Dimitrij Bogdanov, Andreas Kleine und Dr. Alberto Bracchi. Danke nicht nur für die fachliche Hilfe: Es hat mit Euch einfach nur Spaß gemacht.

Zum Glück gibt es ein Leben jenseits der Physik. In diesem Zusammenhang möchte ich insbesondere Marc und Judith, Hendrik und Kathrin, Carsten und Monique, Seve und Ingo sowie Horst und Katrin für ihr stetes Interesse und ihre Unterstützung danken. Speziell erwähnen möchte ich das Zeltlagerteam um Nicole: Eine herrliche Art und Weise, den Kopf mit Dingen jenseits des Nanokosmos zu füllen.

Igg säch ok dem Jan Petersen alias Jan the rippler hartlik dank. Auch wenn wir in den vielen gemeinsamen Jahren nie plattdeutsch miteinander gesprochen haben, gab's doch viele andere Gemeinsamkeiten. Die Gitarre und der HSV sind da nur die wichtigsten. Lustig war's gemeinsam im Büro, am REM, am Kicker, in der Kneipe oder sonstwo. Und Spaß bei der Arbeit ist eine Grundvoraussetzung für eine vernünftige Arbeitsatmosphäre, und Spaß kann man mit Jan wahrlich haben. Ich hoffe auf weitere gemeinsame involtiniAbende.

Meiner Prinzessin Maria danke ich nicht nur für die Unterstützung während dieser Arbeit durch zahlreiche Korrekturen und Diskussionen, sondern besonders für ihr Zutrauen, ihre Wärme und ihre Liebe, die mich so glücklich macht.

Ganz besonders danke ich meiner Familie, insbesondere meinen wunderbaren Eltern. Die Unterstützung in allen Lebenslagen und Euer unendliches Verständnis auch in schwierigen Zeiten haben u.a. die Entscheidung zur Promotion und somit diese Arbeit erst möglich gemacht. Danke. 


\title{
Lebenslauf
}

\section{Persönliche Daten}

Name: $\quad$ Markus Esseling

Geburtsdatum: 15.12.1976

Geburtsort: Bad Laer

Familienstand: ledig

Nationalität: deutsch

\section{Schulausbildung und Berufserfahrung}

\author{
1983-1987 Grundschule Dissen \\ 1987-1989 Orientierungsstufe Dissen \\ 1989-1996 Gymnasium Bad Iburg \\ 1996 Abitur
}

1996-1997 Zivildienst in der Schüchtermann-Klinik, Bad Rothenfelde

1997-2003 Studium der Physik an der Universität Göttingen

2001-2003 Diplomarbeit bei Prof. Dr. K. Samwer, Thema: „Struktur und Transporteigenschaften dünner, gesputterter CoFeHfO-Schichten"

2001-2003 studentische Hilfskraft an der Universität Göttingen: Übungsgrupenleiter in Physik 1, Physik 2, Physik für Nebenfächler

seit 2003 wissenschaftlicher Mitarbeiter im SFB 602 „Komplexe Strukturen in kondensierter Materie von atomarer bis mesoskopischer Skala", Teilprojekt A2; Übungsgruppenleiter in Festkörperphysik, Einführung in die Material-und Festkörperphysik sowie Versuchsbetreuer im Forgeschrittenenpraktikum; Anfertigen der vorliegenden Dissertation am I. Physikalischen Institut der Universität Göttingen bei Prof. Dr. K. Samwer 


\section{Weiterbildung}

2001 IFF Ferienschule Jülich: „Neue Materialien für die Informationstechnik"

2003 IFF Ferienschule Jülich: „Fundamentals of Nanoelectronics“

2005 IFF Ferienschule Jülich: „Magnetism goes nano“

\section{Kontakt}

Markus Esseling

Auf dem Heidbrink 6

49201 Dissen a.T.W.

messeli@gmx.de 